MASSACHUSETTS INSTITUTE OF TECHNOLOGY

Laboratory for Nuclear Science Technical Report

\title{
THE POLARIZATION OF PHOTONEUTRONS FROM THE GIANT RESONANCE
}

\author{
FREDERICK A. HANSER
}

APRIL 1967

Report No. MIT - 2098-307 


\section{DISCLAIMER}

This report was prepared as an account of work sponsored by an agency of the United States Government. Neither the United States Government nor any agency Thereof, nor any of their employees, makes any warranty, express or implied, or assumes any legal liability or responsibility for the accuracy, completeness, or usefulness of any information, apparatus, product, or process disclosed, or represents that its use would not infringe privately owned rights. Reference herein to any specific commercial product, process, or service by trade name, trademark, manufacturer, or otherwise does not necessarily constitute or imply its endorsement, recommendation, or favoring by the United States Government or any agency thereof. The views and opinions of authors expressed herein do not necessarily state or reflect those of the United States Government or any agency thereof. 


\section{DISCLAIMER}

Portions of this document may be illegible in electronic image products. Images are produced from the best available original document. 


\section{$M I T-2098 \cdot 387$}

CESTI PRTES

MASSACHUSETTS INSTITUTE OF TECHNOLOGY

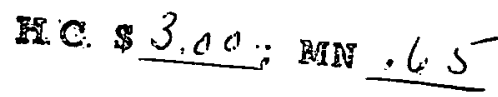

Laboratory for Nuclear Science Technical Report

$\because$

The Polarization of Photoneutrons

from the Giant Resonance

Frederick A. Hanser

April 1967

Report No. MIT-2098-307

Reproduction in whole or in part is permitted for any

Purpose by the U.S. Government. The data and the re-

sults that are presented in this report should not other :

wise be published without prior consultation with the

Laboratory for Nuclear Science.

Researches herein reported have been supported by the Atomic Energy Commission through AEC Contract AT(30-1) -2098 .

This technical report is number 84 in a series of technical reports issued by the Laboratory for Nuclear Science since its inception. 


\section{ÁBSTRACT}

The Polarization of Photoneutrons from the Giant Resonance by Frederick A. Hanser

Submitted to the Department of Physics on January 9, 1967 in partial fulfillment of the requirements for the degree of Doctor of Philosophý.

The polarization of photoneutrons from $\mathrm{O}^{16}, \mathrm{C}^{12}, \mathrm{Ca}^{40}$, and $\mathrm{Pb}^{208}$, emitted at $45^{\circ}$ and $90^{\circ}$ to various energy bremsstrahlung beams, has been measured. The polarizations for $0^{16}$ are about $-0.05 \pm 0.02$ at $90^{\circ}$ and $+0.50 \pm 0.05$ at $45^{\circ}$, for neutrons in the range of 5-10 MeV and for bremsstrahlung from $30 \mathrm{MeV}$ electrons. Polarizations are along $\vec{k}_{\gamma} \times \vec{k}_{n}$, using the Basel convention for the sign. The $90^{\circ}$ measurements indicate that the $0^{16} \mathrm{El}$ giant resonance has some MI and/or E2 contamination. Neglecting this contamination and assuming ground state transitions to $0^{15}$, the $45^{\circ}$ polarization data and the angular distribution results of Verbinski and Courtney can be fit by about equal amounts of $S$ and $D$ wave neutrons with $a$, relative phase difference of about $110^{\circ}$.

The polarizations for $\mathrm{C}^{12}$ are about $0.00 \pm 0.05$ at $90^{\circ}$ and $-0.20 \pm 0.05$ at $45^{\circ}$, for neutrons in the range of $3-10 \mathrm{MeV}$ and for bremsstrahlung from $30 \mathrm{MeV}$ electrons. These results and the angular distribution results of Verbinski and Courtney can not be uniquely explained, as the $\mathrm{El}$ giant resonance for $\mathrm{C}^{12}$ has three amplitudes for ground state transitions to $\mathrm{C}^{11}$, and these amplitudes can fit the measurements in many ways.

The results for $\mathrm{Ca}^{40}$ give about $0.0 \pm 0.1$ at $90^{\circ}$. No polarization measurements were made at $45^{\circ}$. The $\mathrm{Pb}^{208}$ results give about $+0.16 \pm 0.10$ at $45^{\circ}$ and $0.00 \pm 0.10$ at $90^{\circ}$.

Thesis Supervisor: William Bertozzi, Associate Professor of Physics. 


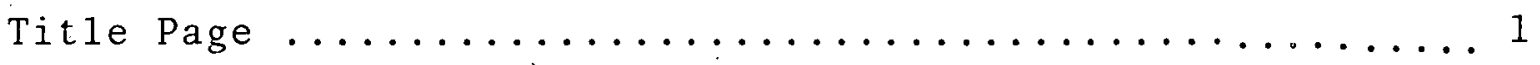

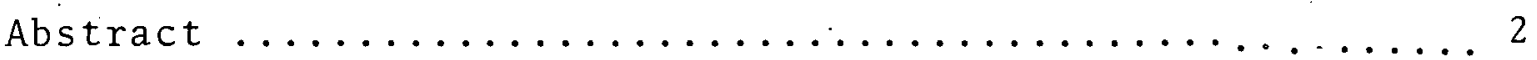

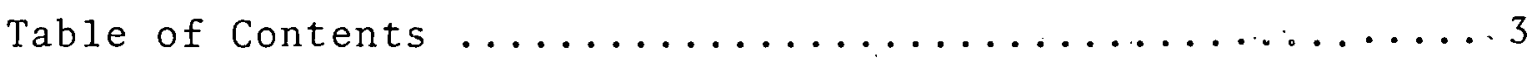

List of Figures $\ldots \ldots \ldots \ldots \ldots \ldots \ldots \ldots \ldots \ldots \ldots \ldots \ldots \ldots \ldots$

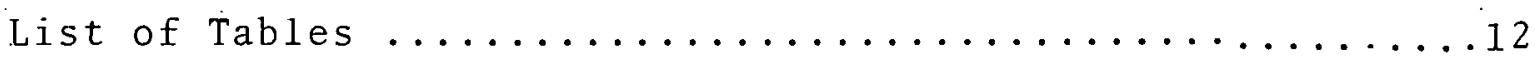

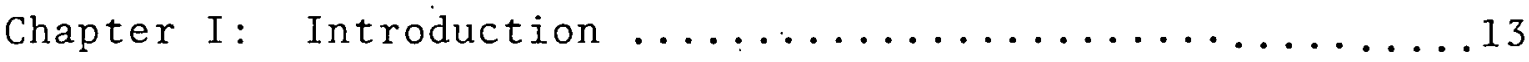

Chapter II: The Polarimeter-Spectrometer ...........23

A. Basic Theoretical and Experimental Aspects

of Neutron Polarimetry-Spectrometry........23

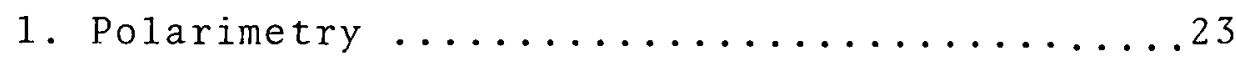

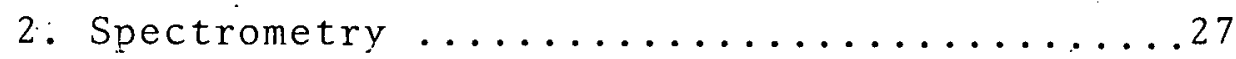

3. Using side-detectors of unequal efficiency..29

4. Electronic timing; coincidence requirements.-31

5. Brief physical description of the polarimeter-spectrometer used............34

B. The Gas Scintillation Counter..............36

1. Physical description of the gas chambers and filling systems...................

2. Cleaning and filling the chambers and

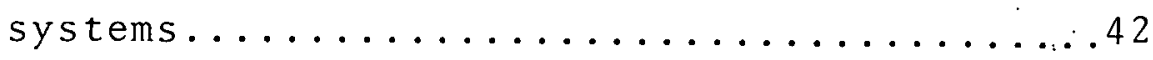

3. Light collection.................46

4. Calibration of the $\mathrm{He}^{4}$ recoil energy vs. PM pulse height.................50 
C. The $\mathrm{He}^{4}$-scattered-neutron Counters (the side-detectors) $\ldots \ldots \ldots \ldots \ldots \ldots \ldots$

1. Description ................ 58

2. Monitoring photomultiplier gains and setting detection thresholds .......6 60

3. Setting the gas-counter to side-detector coincidence timing ...........6 64

D. Electronic Logic .................66

Chapter III: Experimental Procedure .............. 70

A. Physical Irayout ................. 70

B. Polarimeter Alignment ............. 74

C. Taking Data - List of Targets Used .....76

Chapter IV: Method of Data Analysis ............ 82

Introduction.................. 82

A. Calculation of Uncorrected Asymmetries

and Their Statistical Uncertainties..... 83

B. Calculation of the Uncorrected Neutron

Polarization and Spectrum ......... 86

C. Corrections Due to Polarimeter and Tunnel Scattering.................. 89

D. Subtraction of Background ........... 99

E. Subtraction of the Corrections Due to

Scattering in the Photoneutron Target....102

F. Effects of Polarimeter Misalignment.....104

Chapter $V: \quad \operatorname{Results\ldots \ldots \ldots \ldots \ldots \ldots \ldots \ldots \ldots \ldots \ldots \ldots \ldots \ldots \ldots \ldots }$

A. $0^{16}, 45$ degrees, 24 MeV bremsstrahlung ...113

B. $\mathrm{O}^{16}, 45$ degrees, $30 \mathrm{MeV}$ bremsstrahlung ...114 
C. $0^{16}, 45$ degrees, $38 \mathrm{MeV}$ bremsstrahjung ... 115

D. $0^{16} .90$ degrees, $30 \mathrm{MeV}$ bremsstrahlung ... 116

E. $\mathrm{C}^{12}, 45$ degrees. $30 \mathrm{MeV}$ bremsstrahlung ... 116

F. $\mathrm{C}^{12} .45$ degrees, $47 \mathrm{MeV}$ bremsstrahlung ... 117

G. $\mathrm{C}^{12}, 90$ degrees, $50 \mathrm{MeV}$ bremsstrahlung ... 117

H. $\mathrm{Ca}^{40}, 90$ degrees, $28 \mathrm{MeV}$ bremsstrahlung.. 117

I. $\mathrm{Pb}^{208}, 45$ degrees, $28 \mathrm{MeV}$ bremsstrahlung.. 118

J. $\mathrm{Pb}^{208} .90$ degrees, $21 \mathrm{MeV}$ bremsstrahlung.. 118

Chapter VI: Response Function of the Polarimeter:

Corrections for Scattering by the Polarimeter and Tunnel: Corrections for Scattering within the Photoneutron Target............. 144 A. Calculation of the Corrections for

Scattering by the Polarimeter and Tunnel. 144

1. General description of the scattering corrections ................. 144

2. Calculation of the corrections for scattering from the cylinder wal1s... 156

3. Calculation of the corrections for scattering from the flanges-multiple scattering effects ............ 157

4. Calculation of the corrections for scattering from the tunnel wall......159

5. Final form of the scattering correction matrices, and their uncertainty ..... 160

B. Measurement of the Corrections for Polarimeter Scattering for $14.7 \mathrm{MeV}$ and $2.86 \mathrm{MeV}$ Neutrons................. 161

1. Results of the measurements .......161 
2. Method of making the measurements ... 168

C. Corrections for Scattering Within the

Photoneutron Target .............. 182,

Chapter VII: Discussion of the Results and Conclusions 191

A. General Considerations on Photonuclear

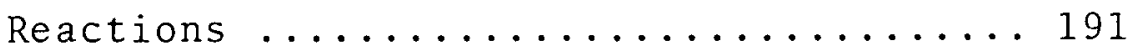

B. Interpretation of the $\mathrm{O}^{16}(r, n)$ Results .. 193

C. Interpretation of the $\mathrm{C}^{12}(r, n)$ Results . 202

D. Interpretation of the $\mathrm{Pb}^{208}(\gamma, n)$ Results 205

Appendix I: Electronic Circuit Ciagrams ........ 207

Appendix II: Calculation of $\overline{\mathrm{P}_{\mathrm{He}}\left(\theta_{0}, \mathrm{E}_{\mathrm{n}}\right)}$ and $\overline{\mathrm{S}_{\mathrm{He}}\left(\theta_{0}, \mathrm{E}_{\mathrm{n}}\right)} \quad 214$ Appendix III: Equations for $\Delta \mathrm{P}_{0} 16$ and $\Delta \mathrm{N}_{016} \ldots \ldots \ldots 224$ Appendix IV: Formulas for the Angular Dependence of the.

$(\gamma, n)$ Cross Section and Neutron Polarization, Including $E 1, M 1$ and E2 Transitions $\ldots . .226$

Appendix V: Calculation of the Neutron Polarization for a Transition to the 6.17 MeV Excited

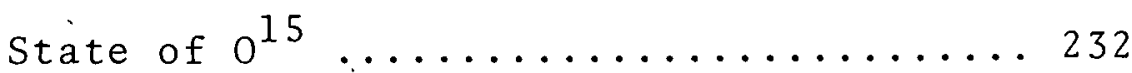

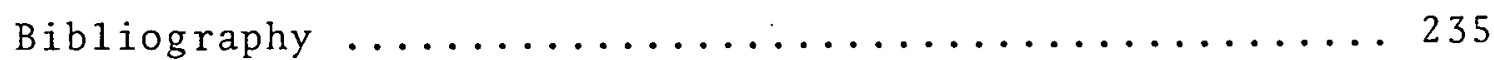

Acknowledgment $\ldots \ldots \ldots \ldots \ldots \ldots \ldots \ldots \ldots \ldots \ldots \ldots \ldots \ldots$

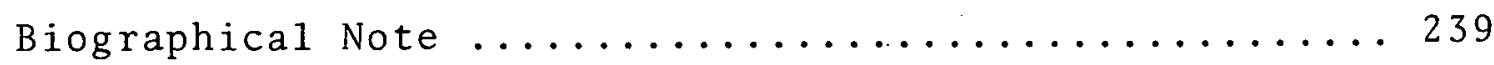




\section{LIST OF FIGURES}

Figure

II-1 Geometry of the Polarization Analysis Scattering $\ldots \ldots \ldots \ldots \ldots \ldots \ldots$

II-2 Basic Structure of the PolarimeterSpectrometer ............... 35

II-3 Aluminum Gas Chamber .......... 37

II-4 Gas Filling System for the Aluminum

Gas Chamber ................. 38

II-5 Stainless Stee1 Gas Chamber ........ 40

II-6 Gas Filling System for the Stainless Steel

Gas Chamber ............... 41

II-7 Layout for Irradiating the Polarimeter

With $14.7 \mathrm{MeV}$ Neutrons .......... 52

II-8 Plots of Po ${ }^{210}$ alpha 'calibration and of

the He ${ }^{4}$ Recoil Calibration ........ 53

II-9 Plot of Slope Ratio vs. Helium Pressure 55

II-10 Plot of Slope Ratio vs. Xenon Pressure 56

II-11 Proton Recoil Neutron Detectors ...... 59

II-12 Mounting Plate for Side-Detectors Used with the Aluminum Gas Chamber ....... 61

II-13 Po 210 Spectrum in a Side-Detector, Illustrating the Threshold ........ 63

II-14 Illustration of the Pulse Relationship at the Input to the Coincidence Circuits 65

II-15 Pólarimeter-Spectrometer Electronic Circuit $\operatorname{Logic} \ldots \ldots \ldots \ldots \ldots \ldots \ldots$ 
Figure

III-1 Physical Layout of the Polarization

Experiments ............... 71

III-2 Bremsstrahlung Source and Electron

Beam Stopper................ 72

II-3 Optical Method for Aligning the

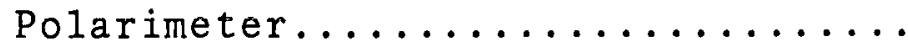

IV-1 Plots of the Four sets of $\left(\mathrm{P}_{4} \cdot \mathrm{N}_{4}\right)$ and of $\left(\bar{P}_{4}, \bar{N}_{4}\right) \ldots \ldots \ldots \ldots \ldots \ldots \ldots \ldots$

V-1 Oxalic Acid, $45^{\circ}, 24 \mathrm{MeV}$ bremsstrahlung

Results ................. 120

V-2 $\quad O^{16}(\gamma, n)$ Neutron Polarization, $45^{\circ}, 24$

MeV bremsstrahlung, Final Results .... 121

V-3 $\mathrm{H}_{2} \mathrm{O} \cdot 45^{\circ}, 30 \mathrm{MeV}$ bremsstrahlung Results 122

V-4 $\quad \mathrm{O}_{2}$ (1iquid), $45^{\circ}, 30 \mathrm{MeV}$ bremsstrahlung

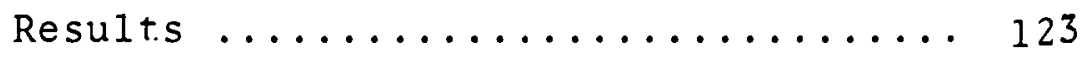

V-5 Oxalic Acid, $45^{\circ}, 30 \mathrm{MeV}$ bremsstrahlung Results.......................... 124

V-6 $\quad \mathrm{O}^{16}(\mathrm{\gamma}, \mathrm{n})$ Neutron Polarization, $45^{\circ}, 30 \mathrm{MeV}$ bremsstrahlung, Final Results ...... 125

V-7 $\quad \mathrm{H}_{2} \mathrm{O}, 45^{\circ}, 38 \mathrm{MeV}$ bremsstrahlung Result.s. 126

V-8 $\quad 0^{16}(\gamma, n)$ Neutron Polarization, $45^{\circ}, 38 \mathrm{MeV}$ bremsstrahlung, Final Results ....... 127

V-9 $\mathrm{H}_{2} \mathrm{O}, 90^{\circ}, 30 \mathrm{MeV}$ bremsstrahlung Results. 128

V-10 Oxalic Acid, $90^{\circ}, 30 \mathrm{MeV}$ bremsstrahlung

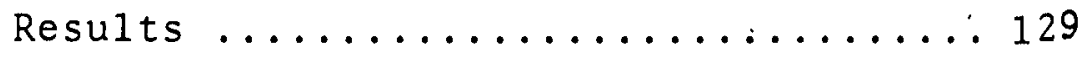


Figure

V-11 $\mathrm{O}^{16}(\gamma, \mathrm{n})$ Neutron Polarization, $90^{\circ}, 30$

MeV bremsstrahlung, Final Results....... 130

V-12 $\mathrm{C}^{12}, 45^{\circ}, 30 \mathrm{MeV}$ bremsstrahlung Results,

taken with the Stainless Steel Gas Chamber 131

V-13 $\mathrm{C}^{12}, 45^{\circ}, 30 \mathrm{MeV}$ bremsstrahlung Results,

taken with the Aluminum Gas Chamber ..... 132

V-14 $\mathrm{C}^{12}(\gamma, n)$ Neutron Polarization, $45^{\circ}, 30 \mathrm{MeV}$

bremsstrahliung, Final Results ........133

V-15 $\mathrm{C}^{12},: 45^{\circ}, 47 \mathrm{MeV}$ bremsstrahlung Results... 134

V-16 $C^{12}(r, n)$ Neutron Polarization, $45^{\circ}, 47 \mathrm{MeV}$

bremsstrahlung, Final Results........ 135

V-17 . . $\mathrm{C}^{12}, 90^{\circ}, 50$ MéV bremsstrahlung Results . 136

$\mathrm{V}-18 \mathrm{C}^{12}(\mathrm{\gamma}, \mathrm{m})$ Neutron Polarization, $90^{\circ} .50 \mathrm{MeV}$

bremsstrahlung, Final Results, and Results

of S. Kowalski for $30 \mathrm{MeV}$ bremsstrahlung...137

V-19 $\mathrm{Ca}^{40}, 90^{\circ}, 28 \mathrm{MeV}$ bremsstrahlung Results ..138

V-20 $\mathrm{Ca}^{40}(r, \mathrm{n})$ Neutron Polarization, $90^{\circ}, 28 \mathrm{MeV}$

bremsstrahlung, Final Results..........139

V-21 $\mathrm{P}_{\mathrm{b}}{ }^{208}, 45^{\circ}, 28 \mathrm{MeV}$ bremsstrahlung Results..140

$\mathrm{V}-22 . \mathrm{P}_{\mathrm{b}}^{208}(\mathrm{\gamma}, \mathrm{n})$ Neutron Polarization, $45^{\circ}, 28$

$\mathrm{MeV}$ bremsstrahlung, Finat Results .......141

V-23 $\mathrm{P}_{\mathrm{b}} 208,90^{\circ}, 21 \mathrm{MeV}$ bremsstrahlung Results..142

V-24 $\quad \mathrm{P}_{\mathrm{b}}{ }^{208}(\gamma, \mathrm{n})$ Neutron Polarization, $90^{\circ}, 21$

MeV bremsstrahlung, Final Results ........143.

VI-1 Geometry of the General Case for the

Polarimeter Scattering Corrections .......145 
Figure

VI-2 Measured and Ideal Response for 14.7 MeV Neutrons ................ 146

VI-3. Geometry of the General Case when the Neutron Scatters first from the Gas

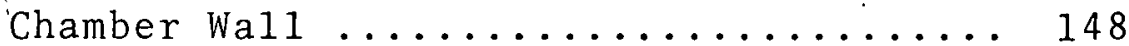

VI-4 Response for Scattering $14.7 \mathrm{MeV}$ Neutrons from $\mathrm{He}$ in the Aluminum Gas Chamber .... 162

VI-5 Response for Scattering $14.7 \mathrm{MeV}$ Neutrons from He in the Stainless Steel Gas

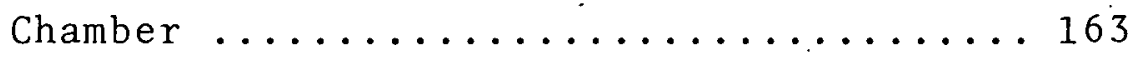

VI-6 Response for Scattering $2.86 \mathrm{MeV}$ Neutrons from He in the Stainless Steel Gas

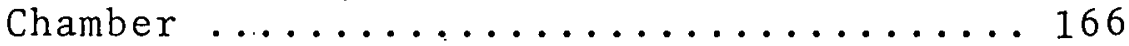

VI-7 Cylinder Component of the Response for Scattering $14.7 \mathrm{MeV}$ neutrons from He in the Stainless Steel Gas Chamber ....... 167 VI-8 Measured Response for Scattering $14.7 \mathrm{MeV}$ Neutrons from $X e$ in the Stainless Steel

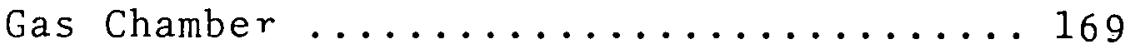

VI-9 Measured Total Response for Scattering 14.7 MeV Neutrons for the Stainless Steel Gas

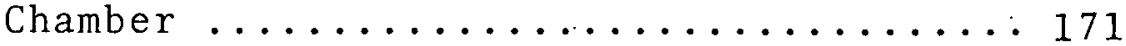

VI-10 Neutron Transmission by 5 " of Wax...... 175 VI-11 Attenuation of Polarimeter Response as a Function of Thickness of Wax in the 14.7 MeV Neutron Beam ..................... 176

VI-12 Response of a Plastic Scintillator to the $14.7 \mathrm{MeV}$ Neutron Beam ........... 181 
Figure.

VI-13 Polarimeter Response as a Function of Coincidence Circuit velay Timang, using

a 14.7 Mev Neutron beam ........... 183

$V_{1-14}$ Geometry tor Ścattering witnin tne

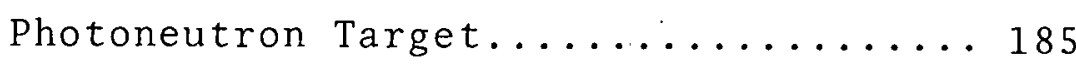

VII-1 Experimental and Theoretical polarizations of $0^{16}(r, n)$ Neutrons at $45^{\circ} \ldots \ldots \ldots 197$

VII-2 Comparison of Experimental $0^{16}(r, n)$ Neutron Polarizations at $45^{\circ}$ and $90^{\circ} \ldots 199$ 


\section{LIST OF TABLES}

Table III-1

List of Photoneutron Targets Usea for polarization Measurements ...

Table IV $\cdots 1$

$\mathrm{He}^{4}(11, \mathrm{n}) \mathrm{He}^{4}$ Polarization Error

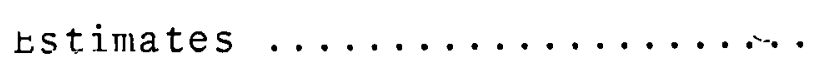

Table IV-2

Asymmetries. Introduced by Dis-

Placing the Polarimcter Perpen-

dicular to the Neutron beam..... 110

Taple IV-3

Asymmetries Introduced by Rotating

the Polarimeter About the Gas

Chamber Axis ................ 111

lable IV-4 The Experimental and Measured

Asymmetries for a I" Displacement,

and for a $2^{\circ}$ Rotation......... 112

Table V-1

Tabulation of the Plots of the Photoneutron Polárization Results 119

Table VI-1

Fraction of Inelastically Scattered

Neutrons Having a Given Energy .. 155

Table VI-2

Gamma Ray Energies and Multiplicities

Used with Inelastic Neutron Scattering

..................... 155 


\section{CHAPTER I}

\section{INTRODUCTION}

The experimental study of photon absorption reveals that nuclei exhibit an electric dipole (E1) giant resonance(1,2). For heavy nuclei the energy of this resonance is given approximately by the formula $80 \mathrm{~A}^{-1 / 3} \mathrm{MeV}{ }^{(2)}$ and has a range in width of 3 to 8 $\mathrm{MeV}$. For light nuclei the giant resonance occurs at 20-25 MeV, with a width comparable to that for heavy nuclei. In the heavy nuclei the giant resonance has little structure on a scale of $1 \mathrm{MeV}$ except for a double peak shape exhibited by the deformed nuclei. On the same scale the light nuclei show considerable structure for the giant resonance, often having several prominent peaks. If the energy resolution is made smaller then both types of nuclei display a fine structure superimposed over the basic giant resonance.

It is found that the heavy nuclei completely exhaust the dipole sum rule for velocity and exchange independent potentials, which predicts that $\int_{0} \sigma E=0.0 .6 \mathrm{NZ} / \mathrm{A} \mathrm{MeV}$-barns, the experimental cross section integrated to about $30 \mathrm{MeV}$ yielding approximately 1.4 times this value ${ }^{(2)}$. For light nuclei only $1 / 3$ to $1 / 2$ of the dipole sum rule seems to be included in the photon absorption below $30 \mathrm{MeV}$.

Theoretical calculations which attempt to describe this giant resonance generally fall into two categories, those which use the hydrodynamic models, and those which use the independent particle model (IPM). The calculations with the collective or 
hydrodynamic model view the nucleus as neutron and proton fluids which oscillate against each other. An early calculation using one of the collective models proposed by Goldhaber and Teller (3) in which the surface neutrons and protons remain fixed with respect to each other and the fluids undergo density changcs because of their vibration, gave the correct dependence of the giant resonance energy of heavy nuclei on their mass number, $\mathrm{E}_{\mathrm{GR}}=\frac{\sqrt{4 \mathrm{NZ}}}{\mathrm{A}} 60 \mathrm{~A}^{-1 / 3} \mathrm{MeV}(4)$. One can also explain the double peak in the giant resonance of deformed nuclei by coupling the El oscillations to the deformations. Experimental measurement of these peaks then enables the quadruple moment of the nucleus to be inferred ${ }^{(2)}$ and yields values in agreement with those found by other techniques.

The present versions of the collective models do not describe all the structure of the giant resonance in light nuclei, nor in general do they describe the decay of the giant resonance by particle emission. Other mechanisms must be introduced to describe the several peaks of the giant resonance in light nuclei, and to describe the de-excitation of the giant resonance by neutron and proton emission.

A second type of calculation uses the independent particle model (IPM). The early versions of this model were summarized in their application to the giant resonance by wilkinson ${ }^{(5)}$ in 1956. These models picture the neutrons and protons of a nucleus as being bound in a potential well with the giant resonance being formed by single-particle absorptions. This provided a mechanism for producing the structure of the giant resonance, but gave the wrong energy unless an ad hoc assumption was used to introduce an effective nucleon mass of $m^{*}=\mathrm{m} / 2$. 
The first use of the IPM to calculate the structure of the giant resonance for $\mathrm{O}^{16}$ was by Elliott and Flowers ${ }^{(6)}$ who calculated the $1^{-}$excited states and their electric dipole absorption strength. When the two dominant absorption states are assigned a width of $1 \mathrm{MeV}$ so they can be compared with experiment ${ }^{(1)}$, the results resemble the gross structure of the $0^{16}$ photon absorption cross section measured by Burgov et al, (7) even though the experimental results show several peaks in a region where Elliott and Flowers predict only two.

More recently Brown and others $(8-13)$ have made calculations with the IPM using single-particle excitations with a residual particle-hole interaction to account for collective effects. Their calculations have had moderate success in describing the giant resonance in $\mathrm{O}^{16}, \mathrm{C}^{12}$ and $\mathrm{Ca}^{40}$, giving reasonable energies and relative absorption strengths to the two or three most prominent $1^{-}$levels for the nuclei. The Brown or Elliott and Flowers models, however, still need addional information to describe particle de-excitation of the giant resonance, since the levels are calculated using bound state wave functions. To describe neutron and photon emission from the giant resonance states requires fitting continuum wave functions to the bound state.wave functions, and the precise manner of doing.this requires some additional assumptions.

Some recent calculations for $0^{16}$ continuum wave functions have been made using finite wells, and the angular distribution and polarization of the outgoing neutrons and protons have been calculated $(14,15,16)$. The results, portions of which will be described in more detail in Chapter VII, give moderate agreement 
with the energy and some of the shape of the giant resonance, including the particle emission spectra, but fail in most of the finer structure $(15,16)$. Thus theoretical work has progressed to the point where it attempts to describe the state of the outgoing particles from the decay of the giant resonance.

Two nuclei which have received extensive experimental and theoretical investigation, and are the main subject for this thesis, are $0^{16}$ and $\mathrm{C}^{12}$. For $0^{16}$ the total gamma absorption cross'section has been measured (7) as well as the (e, $\left.{ }^{\prime}\right)$ cross section at $180^{\circ}$ for excitation of the giant resonance $(17-21)$. The total photoneutron production cross section has been measured $(22,23)$ as have the spectra $(24,25)$ and angular distribution ${ }^{(26)}$ of these photoneutrons. The angular distribution of the ground state protons from $0^{16}(\gamma, p)$ has been measured direct $1 y(27)$ and by the inverse $\mathrm{N}^{15}\left(\mathrm{p}, \gamma_{\mathrm{O}}\right)$ reaction $(28-32)$ using detailed balance. The $\mathrm{O}^{16}\left(\mathrm{e}, \mathrm{e}^{\prime} \mathrm{p}\right)$ proton $\equiv n g u l a r$ distribution and energy spectra have been measured using incident electrons of $30 \mathrm{MeV}^{(33)}$.

For $\mathrm{C}^{12}$ the total gamma absorption ${ }^{(34)}$ and neutron production (35) cross sections have been measured as have the $\mathrm{C}^{12}\left(\mathrm{e}, \mathrm{e}^{\prime}\right)$ cross section at $180^{\circ}(17)$. The angular distribution of neutrons from $\mathrm{C}^{12}(\gamma, n)$ has been measured by time-of-flight $(26)$ and also for the integrated neutron yield for $\mathrm{E}_{\mathrm{ex}}>22 \mathrm{MeV}^{(36)}$. The $\mathrm{C}^{12}(\gamma, \mathrm{p})$ angular distribution has been measured directly ${ }^{(43)}$ and by the inverse $\mathrm{B}^{11}\left(\mathrm{p}, \gamma_{\mathrm{O}}\right)$ reaction $(37,38)$ using detailed balance. The $\mathrm{C}^{12}\left(\mathrm{e}, \mathrm{e}^{\prime} \mathrm{p}\right)$ proton angular distribution and energy spectra have been measured using incident electrons of $30 \mathrm{MeV}$ and $24.5 \mathrm{MeV}^{(33)}$.

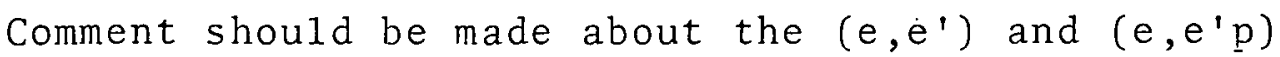
experiments $(17-21,33)$. The electron interacts with the nucleus 
is related to photon absorption. The principal differences are that an electron has a longitudinal field in addition to the transverse component, and for a given excitation it can impart a variable momentum transfer to the nucleus. Theoretical calculations have been made $(39-42)$ which allow the multipolarity of a peak in the $\left(e, e^{\prime}\right)$ cross section to be determined by measuring its strength as a function of the momentum transfer to the nucleus $(18,19,17)$. For $180^{\circ}$ electron scattering only the transverse part of the electron's field takes part and the interaction proceeds as in the photon case, the matrix elements however, being evaluated at higher momentum transfers.

Where momentum transfers are not much greater than for photons with the giant resonance energy, (as when the electron's Energy $\left.\simeq E_{G R}\right)$, the (e,e'p) cross section should give the same information as the $(\gamma, p)$ cross section ${ }^{(44)}$. In particular, Dodge and Barber ${ }^{(33)}$, used the virtual photon spectrum from electrons of 30 and $24.5 \mathrm{MeV}$ to calculate the $(r, p)$ cross section from the measured (e,e'p) yields.

There is good agreement among the different experiments on the detailed structure of the giant resonance region of both $0^{16}$ and $\mathrm{C}^{12}$. In the case of $0^{16}$ the giant resonance is for the most part concentrated in two peaks at about 22.2 and $24.5 \mathrm{MeV}$, with several smaller peaks also apparent. The $0^{16}\left(e, e^{\prime} p\right)$ proton angular distributions of Dodge and Barber (33) show a slight backward peaking below, and a more pronounced forward peaking above, each of the two dominant peaks of the giant resonance, which they found to be at 22.30 and $24.35 \mathrm{MeV}$. This is interpreted as the interference of an E2 level at higher energy, with the 
dominant El resonance. The $\mathrm{N}^{15}\left(\mathrm{p}, \mathrm{Y}_{\mathrm{o}}\right)$ angular distributions of Earle et al (29) are in general agreement with the (e,e'p) measurements. The $0^{16}(\gamma, n)$ neutron angular distributions, although fragmentary, $(26,36)$ are consistent with a pure El resonance. The $0^{16}\left(\mathrm{e}, \mathrm{e}^{\prime}\right)$ results of Goldemberg and Barber (17) for the main part of the giant resonance have been compared with calculations by Lewis (41) using the Brown model (IPM) and the Goldhaber-Teller model (collective). The results appear to support the Brown model calculations and disagree with the Goldhaber-Teller model calculations. However, more recent work by Lewis (45) suggests that if spin effects are included in the Goldhaber-Teller collective mode1, the disagreement of experiment and calculation for the $\mathrm{O}^{16}\left(\mathrm{e}, \mathrm{e}^{\prime}\right)$ giant resonance is reduced.

For $\mathrm{C}^{12}$ the giant resonance is concentrated in a large peak at about $23 \mathrm{MeV}$, which appears to be composed of two or more overlapping components. There is a smaller peak at about 25.5 MeV. The $\mathrm{C}^{12}$ (e,e'p) proton angular distributions of Dodge and Barber (33) show a slight forward peaking for the giant resonance. The photoproton $(43)$ and inverse $B^{11}\left(p, \gamma_{0}\right)(37,38)$ angular distributions also show a slight forward peaking. The photoneutron angular distribution measurements $(26,36)$ are consistent with an E1 resonance. The $\mathrm{C}^{12}\left(\mathrm{e}, \mathrm{e}^{\prime}\right)$ results of Goldemberg and Barber (17) support the Brown model calculations of Lewis (41). The same comments concerning spin effects apply as in the case of the $0^{16}\left(e, e^{\prime}\right)$ results.

The principal conclusions from the experimental and theoretical work on $\mathrm{O}^{16}$ and $\mathrm{C}^{12}$ is that the giant resonance is predominantly E1, with a small amount of opposite parity, possibly E2, interference. 
The IPM using the particle-hole formalism of Brown $(8,9)$, or for that matter the calculational scheme of Elliott and Flowers $(6)$, provides a better theoretical picture of the giant resonance with its structure than the collective model. The Brown model generally gives the energy and relative dipole absorption strengths of the dominant peaks in the giant resonance with reasonable agreement with experiment, and gives the best description of the $180^{\circ}$ electron scattering results $(17,41)$.

As already mentioned, the IPM models require additional information to describe the particle emission widths of the giant resonance. The models for $0^{16}$ in ref.(14), (15) and (16) are the beginning of this extension. Additional experimental work is required to check the results of these new calculations and provide information for future calculations. A measurement of the polarization of the photoparticles yields additional information about the giant resonance. The polarization provides information about the state of the outgoing particle which complements the angular distribution and total cross section since it provides a different linear combination of the amplitudes and phases involved in the process.

By measuring either the angular distribution or the polarization of the neutron in a reaction such as $\mathrm{O}^{16}(\gamma, n)$ it is in general not possible to specify the state of the outgoing neutron completely. The case of $0^{16}(\gamma, n)$ is particularly simple in the El approximation. The $0^{16}$ ground state is $0+$, and after El photon absorption the excited state is $1^{-}$. The decay to the $1 / 2^{-}$ground state of $0^{15}$ emits a combination of $\mathrm{s}_{1 / 2}$ and $\mathrm{d}_{3 / 2}$ neutron waves. For this case the cross section will be determined 
by three parameters, the amplitudes of these two waves and their phase difference. However, only two terms enter into the expansion for the cross section,

$$
\frac{\mathrm{d} \sigma}{\mathrm{d}_{\Omega}}=\mathrm{A}+\mathrm{B} \mathrm{P}_{2}(\cos \theta) \quad \mathrm{I} .1
$$

Equations relating $A$ and $B$ to the amplitudes and phase difference are given in Chapter VII.

The $0^{16}(\gamma, n)$ cross section measurements of Verbinski and Courtney ${ }^{(26)}$ gave an angular distribution consistent with pure d wave emission, and on this basis they concluded that the neutrons were pure d wave. However, by choosing a suitable phase difference it is possible to fit the angular distribution and also have a large $s$ wave component. Thus their conclusion of pure $d$ wave. neutron emission is only tentative.

The $\mathrm{N}^{15}\left(\mathrm{p}, \dot{\gamma}_{\mathrm{o}}\right)$ results of Earle et al ${ }^{(29)}$ lead them to conclude that the 22 and $24.5 \mathrm{MeV}$ giant resonance peaks go primarily by $d$ wave proton capture, but this is also only tentative as the interference term can lead to the same angular distribution with a large $s$ wave amplitude.

Additional information about the state of the outgoing neutron may be obtained from the polarization. For the case of $\checkmark 0^{16}(\gamma, n)$ the polarization yields a third independent quantity and gives a more complete specification of the state of the outgoing neutron.

The case for $\mathrm{C}^{12}(\gamma, \mathrm{n})$ is not as simple as $0^{16}$, since the $\mathrm{C}^{12}$ ground state of $\mathrm{O}^{+}$leads to a $1^{-}$state after $\mathrm{E} 1$ photon absorption, and the decay is to the $3 / 2^{-}$ground state of $\mathrm{C}^{11}$. This allows the emission of $s_{1 / 2}, d_{3 / 2}$, and $d_{5 / 2}$ wave neutrons, though the angular distribution and polarization yield only three 
independent quantities, as in the case of $0^{16}$. For $C^{12}(\gamma, n)$ the neutron state is determined by three amplitudes and two phase differences, and thus angular distribution and polarization measurements using unpolarized photon beams cannot uniquely. specify the neutron state.

The ${ }_{r} \mathrm{C}^{12}(\gamma, \mathrm{n})$ angular distribution of Verbinski and Courtney (14) is consistent with d wave neutron emission, and they concluded that such is the case. However, on the basis of the preceding arguments it is seen that this need not be true. A simple test would be to measure the polarization of the $\mathrm{C}^{12}$ photoneutrons at $45^{\circ}$ to the photon beam, as any non-zero polarization implies the existence of at least two waves.

In this thesis are presented the results of polarization measurements on photoneutrons from $\mathrm{O}^{16}, \mathrm{C}^{12}, \mathrm{Ca}^{40}$, and $\mathrm{Pb}^{208}$, emitted at $45^{\circ}$ and $90^{\circ}$ to an unpolarized photon beam. For $0^{16}$ the results indicate that a large amount of $s$ wave neutrons is required, in contrast to other conclusions that mostly d wave neutrons are present. For the case of $\mathrm{C}^{12}$ the polarization and angular distribution for unpolarized photons cannot provide a unique description of the state of the neutrons, but the pólarization results do require the presence of both $s$ wave and d wave neutrons.

For $\mathrm{Ca}^{40}$ polarization measurements were made only at $90^{\circ}$ and do not provide any information about the state of the outgoing neutrons. The approximately zero polarization results are consistent with a pure El giant resonance. The $\mathrm{Pb}^{208}$ results show a small positive polarization at $45^{\circ}$, and are consistent with the angular distribution measurements of Mutchler ${ }^{(58)}$ who found that 
the outgoing neutrons could not be pure $d$ wave but required some $s$ wave interference.

The polarization measurements were made using $\mathrm{He}^{4}$ as the analyzing scatterer. The polarimeter, described in Chapter II, is a modification of the one used by Kowalski ${ }^{(46)}$ in his measurements of the $\mathrm{H}^{2}(\gamma, n)$ polarization at $90^{\circ}$. The method of acquiring the polarization data is presented in Chapter III, while the analysis of the data is described in Chapter IV. The final results are given in the several graphs of Chapter V. Chapter VI describes how various scattering corrections to the data were calculated, and how some of these corrections were experimentally measured. A discussion of the results and the conclusions, with most emphasis on $\mathrm{O}^{16}$ and $\mathrm{C}^{12}$, are given in Chapter VII, where some values for amplitude ratios and relative phase shifts of the neutron waves are given. 
CHAPTER I I

THE POLARIMETER-SPECTROMETER

The chapter contains a discussion of the basic concepts of polarimetry and spectrometry as applied to the $\mathrm{He}^{4}$ instrument used in this experiment. It includes a description of the gas scintillator and system, and the associated counters and electronics. Many of the theoretical results can be applied to scattering from any analyzer, but for the sake of specificity all formulas and discussion will refer to the scattering from a $\mathrm{He}^{4}$ analyzer.

A. Basic Theoretical and Experimental Aspects of Neutron

\section{Polarimetry-Spectrometry}

1. Polarimetry

The polarization of a neutron beam, $\overrightarrow{\mathrm{P}}_{\mathrm{n}}$, is defined as follows. Using an arbitrary direction as an axis of quantization the number of neutrons in the beam with spin states $\pm 1 / 2$ are $N_{ \pm 1 / 2}$ This defines a polarization

$$
p \equiv \frac{N+1 / 2-N-1 / 2}{N+1 / 2+N-1 / 2}
$$$$
\text { I I . } 1
$$

The direction of $\overrightarrow{\mathrm{P}}_{\mathrm{n}}$ is that choice of the axis of quantization which gives the maximum positive value for $\dot{p}$, and the magnitude of $\vec{F}_{n}$ is equal to this maximum positive value. $\vec{P}_{n}$ is specified by three real numbers, e.g. the Cartesian components of $\vec{P}_{n}$, $\left(P_{n x}, P_{n y}, P_{n z}\right)$ 
It will now be shown that scattering the neutron beam from a polarization analyzer can give a measurement of the components of $\overrightarrow{\mathrm{P}}_{\mathrm{n}}$. The geometry of the scattering from the polarization analyzer is drawn in fig. II-1. A neutron beam, - with wave vector $\vec{k}_{\mathrm{n}}$ is incident on a small volume of He. Two small neutron counters (side-detectors) are located symmetrically w.r.t. $\vec{k}_{n}$ and the He volume. $\vec{k}_{n}$, the He volume, and the two side-detectors all lie in the same plane. The two side-detectors are arbitrarily distinguished by labeling them right and left. Some neutrons in the incident beam are scattered by the He through an angle $\left|\theta_{0}\right|$ and strike one of the side-detectors. The angle of scattering for the neutrons is labeled as $+\theta_{0}$ for the right, $-\theta_{0}$ for the left, sidedetector, with the wave vectors of the scattered neutrons being $\vec{k}_{n},\left(\underline{\theta}_{0}\right)$. If $N$ neutrons are incident on the He volume let $\mathrm{N}_{\mathrm{R}}$ and $\mathrm{N}_{\mathrm{L}}$ be the number counted in the right and left sidedetectors respectively. Then a scattering asymmetry is defined by

$$
A_{n} \equiv \frac{N_{R}-N_{L}}{N_{R}+N_{L}}
$$

It is now necessary to relate $A_{n}$, a measured quantity, to a component of the polarization of the incident neutron beam. The $\mathrm{He}^{4}(n, n)$ scattering must conserve parity since the reaction takes place via the strong interactions. Since $\mathrm{He}^{4}$ is a spin zero nucleus, the only axial vectors entering

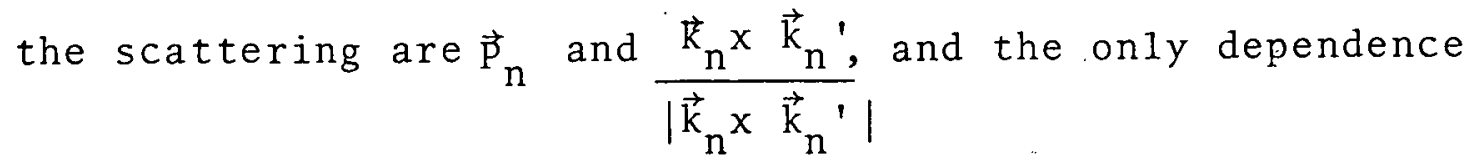




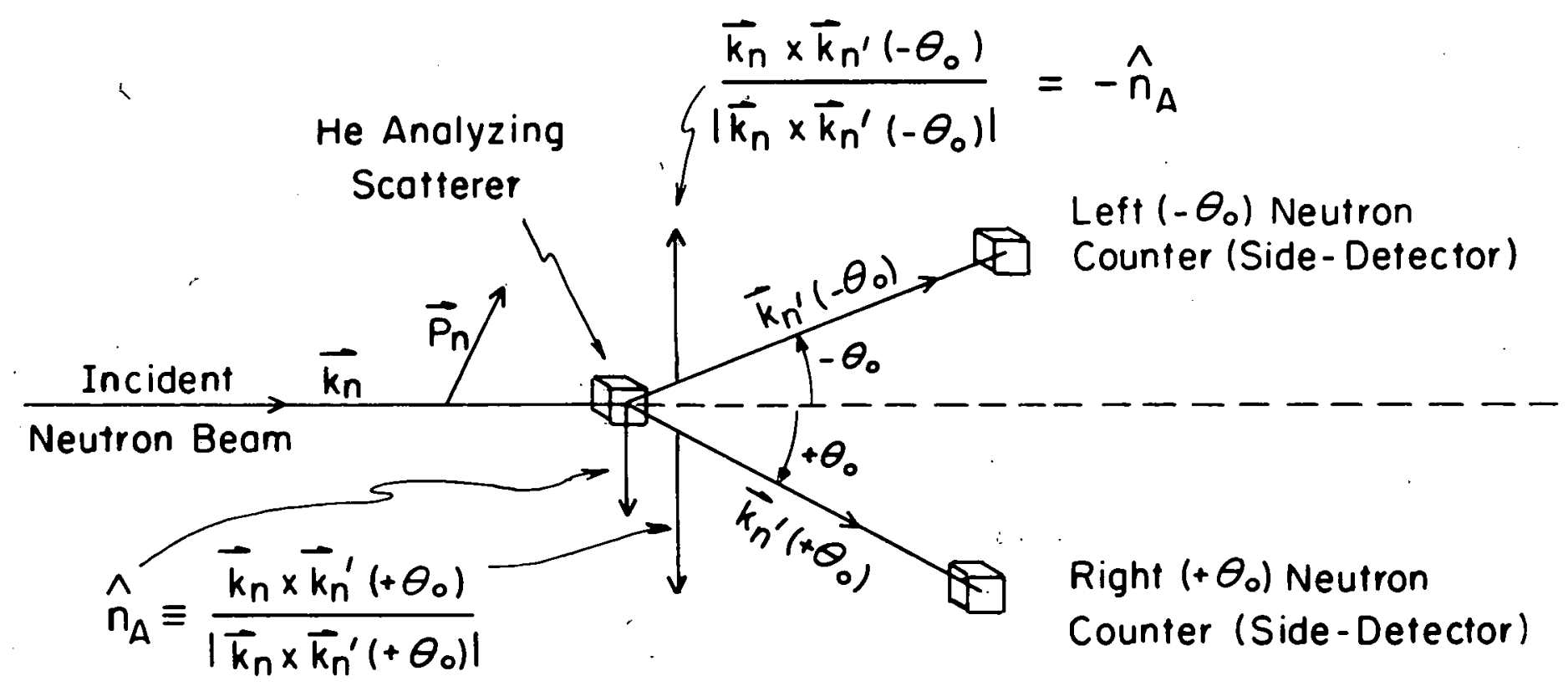

Geometry of the Polarization Analysis Scattering

Fig. II-1 
of the scattering cross-section on $\vec{P}_{n}$ can be via the term $\overrightarrow{\mathrm{P}}_{\mathrm{n}} \cdot \frac{\left(\overrightarrow{\mathrm{k}}_{\mathrm{n}} \times \overrightarrow{\mathrm{k}}_{\mathrm{n}}{ }^{\prime}\right)}{\left(\mathrm{k}_{\mathrm{n}} \overrightarrow{\mathrm{k}}^{\prime}\right)}$. For spin $1 / 2$ particles $\overrightarrow{\mathrm{P}}_{\mathrm{n}}$ is proportional to an average of the expectation value of $\vec{\sigma}$, the Pauli spin matrices, so higher powers of $\vec{p}_{n}$ do not appear since $\sigma_{\mathrm{X}}^{2}=\sigma_{\mathrm{y}}^{2}=\sigma_{\mathrm{z}}^{2}=1$ and $\sigma_{i} \sigma_{j}=\sqrt{-1} \sigma_{k} \quad$, where $i, j, k$, is a cyclic permutation of $x, y, z$. Thus the $\mathrm{He}^{4}(\mathrm{n}, \mathrm{n})$ scattering crosssection has the form

$$
\frac{\mathrm{d}_{\sigma}}{\mathrm{d} \Omega}(\theta)=f(\theta)+g(\theta) \overrightarrow{\mathrm{P}}_{\mathrm{n}} \cdot \frac{\left(\overrightarrow{\mathrm{k}}_{\mathrm{n}} \times \overrightarrow{\mathrm{k}}_{\mathrm{n}}{ }^{\prime}\right)}{\left|\overrightarrow{\mathrm{k}}_{\mathrm{n}} \times \overrightarrow{\mathrm{k}}_{\mathrm{n}}{ }^{\prime}\right|} \quad \text { I I.3 }
$$

where $\theta$ is the angle between $\vec{k}_{n}$ and $\vec{k}_{n}{ }^{\prime}$, and $f(\theta)$ and $g(\theta)$ also depend on $E_{n}$, the incident neutron energy. For the specific cases of scattering at $\pm \theta_{0}$, $\frac{\vec{k}_{n} \times \vec{k}_{n},\left( \pm \theta_{0}\right)}{\left|\vec{k}_{n} x \vec{k}_{n} \cdot\left( \pm_{0}\right)\right|}= \pm \hat{n}_{A} \quad($ see fig. II-1) and there results

1

$$
\frac{d_{\sigma}}{d_{\Omega}}\left( \pm \theta_{0}\right)=f\left(\theta_{0}\right) \pm g(\underbrace{}_{0}) \vec{p}_{n} \cdot \hat{n}_{A}
$$

Then for a given number $N$ of neutrons incident on the $\mathrm{He}$ the number detected right and left are

$$
\begin{aligned}
& N_{R}=N\left(+\theta_{0}\right)=N n\left[f\left(\theta_{0}\right)+g\left(\theta_{0}\right) \vec{P}_{n} \cdot \hat{n}_{A}\right] \\
& N_{L}=N\left(-\theta_{0}\right)=N n\left[f\left(\theta_{0}\right)-g\left(\theta_{0}\right) \vec{P}_{n} \cdot \hat{n}_{A}\right] \text { II. } 5
\end{aligned}
$$

where $n$ is a product of the number of scattering $\mathrm{He}^{4}$ nuclei, the solid angle of the side-detectors, and the detection efficiency of the side-detectors for the scattered neutrons. Using the asymmetry defined earlier there results

$$
A=\frac{N_{R}-N_{L}}{N_{R}+N_{L}}=\vec{P}_{n} \cdot \hat{n}_{A}\left[\frac{g(\theta 0)}{f(\theta 0)}\right] \quad \text { II.6 }
$$


If $\frac{\mathrm{g}\left(\theta_{0}\right)}{\mathrm{f}\left(\theta_{0}\right)} \equiv \mathrm{P}_{\mathrm{He}}\left(\theta_{0}\right)$ then $\mathrm{P}_{\mathrm{He}}\left(\theta_{0}\right)$ is the polarizing power of He for neutrons of energy $E_{n}$ and scattering angle $\theta_{0}$. If $\mathrm{P}_{\mathrm{He}}\left(\theta_{0}\right)$ is known, then

$$
\vec{P}_{n} \cdot \hat{n}_{A}=\frac{A}{P_{H e}\left(\theta_{0}\right)}
$$

and the component of $\vec{P}_{n}$ along $\hat{n}_{A}$ has been measured.

$\mathrm{P}_{\mathrm{He}}\left(\theta_{0}\right)$ is the polarization of the unpolarized neutrons, $\vec{k}_{n}$, after scattering elastically from He at an angle $\theta_{0}$ with a final wave vector $\vec{k}_{n}^{\prime}\left(\theta_{0}\right)$. Using the Basel convention $\mathrm{P}_{\mathrm{He}}\left(\theta_{0}\right)$ is positive when the polarization of the scattered neutron is in the positive sense of $\vec{k}_{n} x \vec{k}_{n}^{\prime}\left(\theta_{0}\right)$.

For the case of photoneutrons from unpolarized gamma-rays and unpolarized targets $P_{n}$ is parallel to $\vec{k}_{\gamma} x \vec{k}_{n}$, so the scattering from the polarization analyzer is set up to make

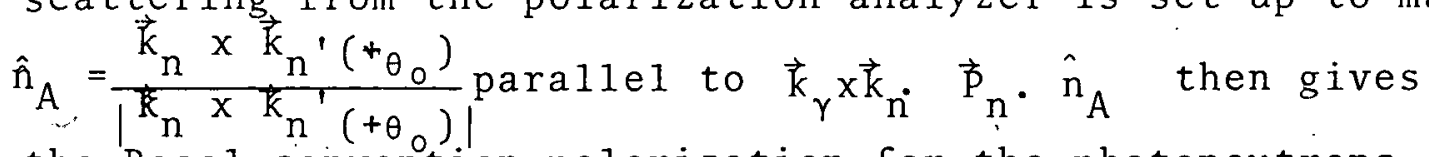
the Basel convention polarization for the photoneutrons, a positive value meaning a polarization in the positive sense of $\vec{k}_{\gamma} \times \vec{k}_{n}$, and a negative value meaning a polarization in the opposite direction.

\section{Spectrometry}

In the preceding sections it was shown how polarization can be measured for a monoenergetic neutron beam. In the more general case a neutron beam has several energy groups and for many experiments the neutron beam may have a continuous energy spectrum. Since the neutron polarization and the $\mathrm{He}^{4}$ polarizing power vary with neutron energy it is necessary to be able to 
separate the side-detector counts as a function of incident neutron energy. This can be done by measuring the recoil energy of the scattering $\mathrm{He}^{4}$ nucleus for a given angle of neutron scattering.

Consider a neutron scattering at an angle $\theta$ from a $\mathrm{He}^{4}$ nucleus at rest. Since $\mathrm{He}^{4}$ has no excited states below $20 \mathrm{MeV}$ all neutrons below this energy must scatter elastically. Then the following result can be derived using classical expressions for energy and momentum conservation.

$$
E_{n}=E_{H e} 4\left(1-\left(\frac{\rho \cos \theta \pm \sqrt{1-\rho{ }^{2} \sin 2} \theta}{1+\rho}\right)^{2}\right)-1 \quad \text { II.8 }
$$

where

$$
\begin{aligned}
& \mathrm{E}_{\mathrm{He}}{ }^{4}=\text { recoil energy of the } \mathrm{He}^{4} \text { nucleus } \\
& \mathrm{E}_{\mathrm{n}} \quad=\text { incident energy of the neutron (energy before } \\
& \text { scattering) } \\
& \begin{aligned}
\rho=\frac{m_{n}}{m_{H_{e}}} & =\text { ratio of neutron mass to } \mathrm{He}^{4} \text { mass }=0.252 \\
\theta & =\text { laboratory angle of scattering, i.e. the }
\end{aligned} \\
& \text { angle between } \vec{k}_{n} \text { and } \vec{k}_{n}^{\prime} \text {, the. neutron wave }
\end{aligned}
$$

Eq. II.8. allows the incident neutron energy to be obtained from a simultaneous measurement of the neutron scattering angle and the $\mathrm{He}^{4}$ recoil energy. Since the physical location of the side-detectors fixes the neutron scattering angle to be $\theta_{0}$ it is only necessary to measure the $\mathrm{He}^{4}$ recoil energy with subsequent detection of the neutron in a side-detector. The proper time sequence for scattering from He and detection by a side-detector is ascertained by electronic requirements 
described in section A4. Using the analysis in eqs. II.2 to II.7 the polarization of the incident neutrons as a function of their energy can then be found.

3. Using side-detectors of unequal efficiency.

In section A-1 an equation was derived for the neutron poiarization in terms of the scattering asymmetry and the $\mathrm{He}^{4}$ polarizing power. This was derived from eqs. II.5. on the assumption of equal detection efficiency for the right and left side-detectors ( $\pm_{\theta_{0}}$, as in fig.II- $)$. In practice the right and left detection efficiencies may be slightly different because detection volumes, detection thresholds, and electronic timing may all be unequal.

Eqs. II.5 may be rewritten, modified to include the more general case and using a more standard notation, to give

$$
\begin{aligned}
& \mathrm{N}_{\mathrm{R}}=\mathrm{N}{ }^{n} \mathrm{R} \frac{\mathrm{d}_{\sigma}}{\mathrm{d} \Omega}\left(\theta_{0}\right)\left[1+\mathrm{P}_{\mathrm{n}} \mathrm{P}_{\mathrm{He}}\left(\theta_{0}\right)\right] \\
& \mathrm{N}_{\mathrm{L}}=\mathrm{N} \eta_{\mathrm{L}} \frac{\mathrm{d}_{\sigma}}{\mathrm{d} \Omega}\left(\theta_{0}\right)\left[1-\mathrm{P}_{\mathrm{n}} \mathrm{P}_{\mathrm{He}}\left(\theta_{0}\right)\right]
\end{aligned}
$$

where

$$
\begin{aligned}
& { }^{n}{ }_{R},{ }_{L}=\text { detection efficiency of the right and left } \\
& \text { side-detectors, respectively. } \\
& \frac{\mathrm{d} \sigma}{\dot{d}_{\Omega}}\left(\theta_{0}\right)=\mathrm{He}^{4},(\mathrm{n}, \mathrm{n}) \text { differential scattering cross- } \\
& \text { section for unpolarized neutrons } \\
& \mathrm{P}_{\mathrm{HE}}\left(\theta_{0}\right)=\mathrm{He}^{4}(\mathrm{n}, \mathrm{n}) \text { polarizing power }
\end{aligned}
$$

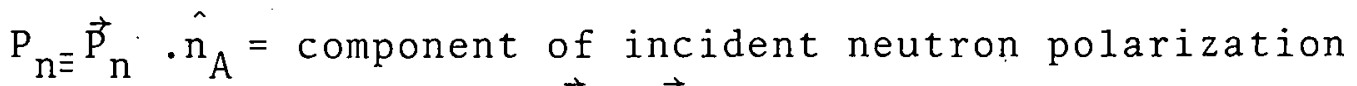

$$
\begin{aligned}
& \text { along } \hat{n}_{A}=\frac{\vec{k}_{n} \times \vec{k}_{n}{ }^{\prime}\left(+\theta_{0}\right)}{\vec{k}_{x} \vec{k}^{\prime}\left(+\theta_{0}\right)} \quad \text { (see fig.II-I) } \\
& \overline{\mathrm{N}}=\text { number of hetutrohs incident on the He. }
\end{aligned}
$$


If the ratio ${ }_{R} / n_{L}$ is known, then

$$
P_{n} P_{H e}\left(\theta_{0}\right)=\frac{{ }^{-} N_{R}-N_{L}\left(n_{R} / n_{L}\right)}{N_{R}+N_{L}\left(n_{R} / n_{L}\right)}
$$

This enables $P_{n}$. to be measured by a single run if another experiment has given ${ }^{n} R / n_{L}$.

The method used in this work to measure ${ }^{n_{R}} /{ }^{n_{L}}$ was to. interchange the right and left side-detectors and make a new polarization measurement. Let the first run be called $A$ and quantities referring to this run be subscripted $A$, the second $B$ with subscripts $B$.

Eq. II.9 becomes

$$
P_{n} P_{H e}\left(\theta_{0}\right)=\frac{N_{R A}-N_{L A}\left(n_{R A} / n_{L A}\right)}{N_{R A}+N_{L A}\left(n_{R A} / n_{L A}\right)} .
$$

for the A run and

$$
P_{n} P_{H e}\left(\theta_{0}\right)=\frac{N_{R B}-N_{L B}\left({ }^{n} \cdot R B / n^{n} \cdot L B\right)}{N_{R B}+N_{L B}\left({ }^{n} R B /{ }^{n} L B\right.}
$$

for the B run. But

$$
\left({ }^{n} R B /{ }^{n} L B\right)=\frac{1}{\left(n_{R A} / n_{L A}\right)} \text {, II.13 }
$$

since the only difference between the runs is an interchange of right and left side-detectors and this interchange is assumed not to affect the detection efficiencies. Now, using eqs. II.11, II.12, and II.13 there results 
where

$$
P_{n} P_{H e}\left(\theta_{0}\right)=\frac{1-\varepsilon}{1+\varepsilon}
$$

$$
\varepsilon=\sqrt{\frac{\mathrm{N}_{\mathrm{RA}} \times{ }_{\mathrm{RB}}^{\mathrm{N}_{\mathrm{R}}}}{\mathrm{N}_{\mathrm{LA} \times{ }^{\mathrm{N}} \mathrm{LB}}}}
$$

We may also obtain.

$$
\frac{{ }^{n_{R A}}}{{ }^{n_{L A}}}=\frac{{ }^{n_{L B}}}{{ }^{n_{R B}}}=\sqrt{\frac{\sqrt{N_{R A} \times{ }^{N_{L B}}}}{N_{L A} \times{ }^{N_{R B}}}}
$$

so that a third, single, polarization run may be analyzed using eqs. II.10 and II.16. Thus a series of polarization measurements requires at least one run pair ( $A$ and $B$ run) with the same neutron beam in order to analyze the entire series. In practice, most polarization measurements are made with run pairs to eliminate effects from long-time changes in the detection efficiency ratio.

Another method of measuring ${ }^{n} \mathrm{R} /{ }^{n} \mathrm{~L}$ is 'to rotate the neutron polarization through 180 degrees, effectively changing the sign of $\mathrm{P}_{\mathrm{n}}$. This is done by having the incident neutron beam travel through a magnetic field, and is most effective for monoenergetic neutron beams.

4. Electronic timing; coincidence requirements.

Two electronic pulses with a definite time relationship are required for the counting of a neutron. The first pulse. comes from the $\mathrm{He}^{4}$ recoil which scattered the incident neutron, and the second, produced an interval of time $\Delta t$ later, comes from the scattered neutron being counted by the side-detector. 
$\Delta t$ is the flight time of the scattered neutron going from the He volume to the side-detector.

For a neutron beam with a spread in energies the value of $\Delta^{t}$, the scattered neutron flight time, will have a corresponding spread, and this, coupled with the electronic rise times and the range in fight times because of the finite dimensions of the He volume and the side-detectors, requires that there be a range in the allowed timing, $\Delta t$, between the He recoil and side-detector pulses. The method of selecting and setting this range in coincidence timing will be described in section C-3. Here we only require knowing that there is a finite spread in coincidence timing, which will be labeled $\tau$ cc . Thus if $\tau_{\text {min }}$ is the minimum delay time between the recoil pulse and the side-detector pulse, at the-coincidence circuit, then when a recoil pulse occurs, in order for this recoil to be counted as a neutron scattering, a side-detector pulse must occur within the time interval $\tau_{\min }$ to ${ }_{\min }+{ }_{\mathrm{cc}}$ later.

The finite coincidence resolving time introduces a new complication. While the coincidences detect real neutron scattering events, they will also include random counts if there are large uncorrelated counting rates in the He gas scintillator and the side-detectors.' If the recoil counting rate is $N_{G C}$ and the side-detector counting rate is $N_{S D}$, then if these two rates are uncorrelated in time the random coincidence rate is

$$
\mathrm{N}_{\text {random }}=\mathrm{N}_{\mathrm{GC}} \mathrm{N}_{\mathrm{SD}} \tau_{\mathrm{CC}}
$$


The $\mathrm{He}^{4}$ recoil counting rate, $\mathrm{N}_{\mathrm{GC}}$, arises mainly from the scattering of neutrons in the incident beam and is largely uncorrelated with side-detector counts. Since a side-detector subtends less than $1 \%$ of a $4 \pi$ solid angle about the He volume the overwhelming number of scattered neutrons are not in the direction of any specified side-detector, even allowing for the angular dependence of the $\mathrm{He}^{4}(\mathrm{n}, \mathrm{n})$ cross-section. The recoil pulses from most of the scattered neutrons are thus uncorrelated with events in any specified side-detector.

The side-detector rate, $\mathrm{N}_{S D}$, arises from natural radioactivity, cosmic rays, photomultiplier noise, and some Po 210 alpha sources mounted in them to help set photomultiplier gains and detection thresholds. These counts are all random w.r.t. the $\mathrm{He}^{4}$ recoils and thus contribute to the rate in eq. II.17. The random counts of eq. II.17 must be measured and subtracted from the coincidence $\mathrm{He}^{4}$ recoil spectra of the sidedetectors. This is done by having a second coincidence circuit for each side-detector. The side-detector pulses going to this coincidence circuit are delayed by about $100 \mathrm{~ns}$ to ensure that they are uncorrelated with real-event $\mathrm{He}^{4}$ recoils. Since the side-detector pulses are delayed this would require the neutron to trigger the side-detector first, whereas the direct neutron beam is incident on the gas-counter and triggers it first. For the He volume to side-detector distances used, $100 \mathrm{~ns}$ would require a scattered neutron energy of less than $1 / 2 \mathrm{MeV}$, and a $1 / 2 \mathrm{MeV}$ neutron could not produce a $\mathrm{He}^{4}$ recoil with energy above the gas scintillator thresholds used. Using the two coincidence circuits each side-detector will 
give two $\mathrm{He}^{4}$ recoil spectra as raw data. The undelayed recoil pulse spectrum will be called the real-plus-random spectrum, from the real-plus-random coincidence circuit. The $100 \mathrm{~ns}$ delayed spectrum will be called the random spectrum, from the random coincidence circuit. The analysis of polarization data. proceeds according to section B-3 using the difference of the real-plus-random and the random spectra.

5. Brief physical description of the polarimeterspectrometer used.

The basic structure of the neutron polarimeter-spectrometer described in this thesis is shown in fig. II-2 . The analyzing He scatterer is contained in a high pressure gas chamber. Scintillations from the $\mathrm{He}^{4}$ recoils are viewed by two photomultipliers which provide the electronic signal for the recoil energy measurement and timing. The side-detectors for the scattered neutrons consist of toluene-filled proton recoil counters. Two photomultipliers view the scintillations in the toluene and provide the neutron counting pulses.

In actual use the polarimeter-spectrometer had four pairs of side-detectors, which increased the efficiency of the polarimeter by making four independent measurements simultaneously. For simplicity most discussion of the polarimeter has centered about only one side-detector pair. 
Photomultipliers viewing Scintillations in He

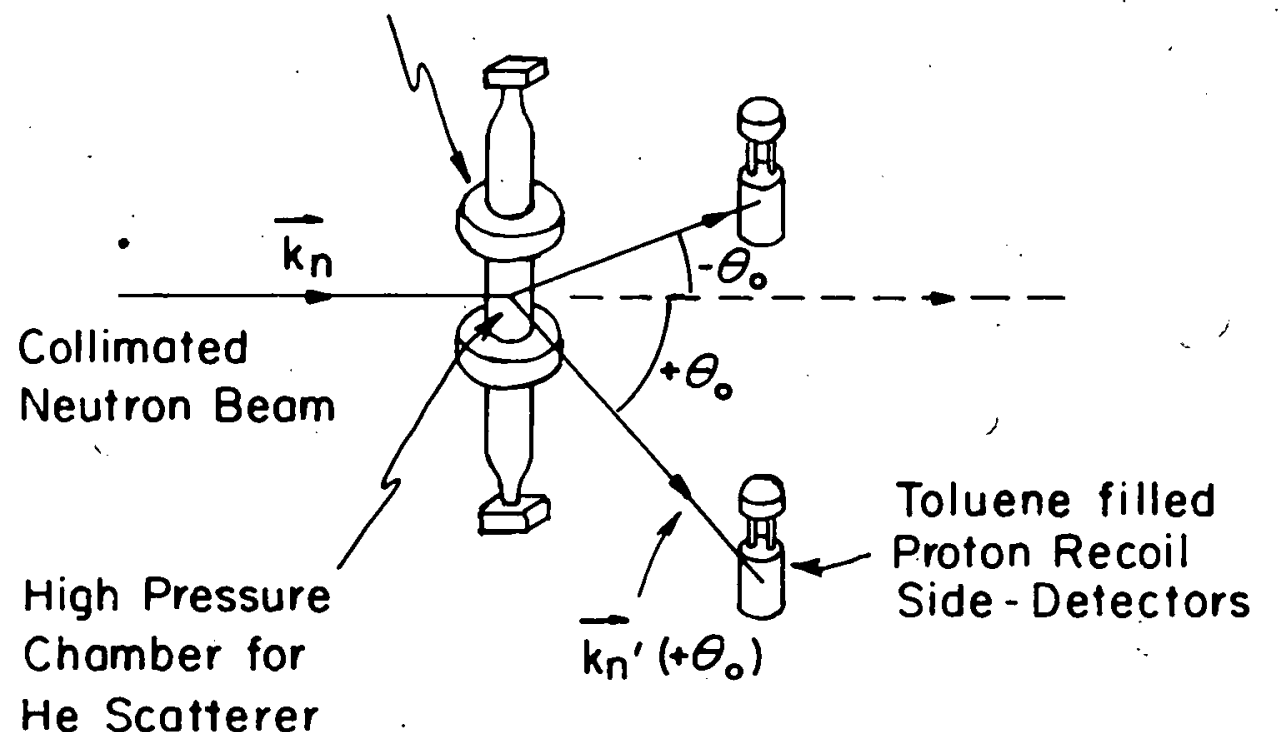

Basic Structure of the Polarimeter-Spectrometer

Fig. II - 2 


\section{B. The Gas Scintillation Counter}

Having explained the basic concepts of the neutron polarimeter-spectrometer, the main component of the instrument, the gas scintillator will now be described. First, a physical description of the gas chambers and filling. systems will be given, including a description of the methods of cleaning the systems. Next the method of light collection and wavelength shifting will be discussed. The description of the gascounter will, end in section $\mathrm{B}-4$ with a discussion of the $\mathrm{He}^{4}$ recoil energy calibration and data will be presented on the stability of this calibration.

1. Physical description of the gas chambers and filling systems

Two gas chambers were used to contain the He-Xe gas scintillator, one made from aluminum and one made from stainless steel. The aluminum chamber was machined out of a forging of heat treated aluminum, type 7075-T6, and is illustrated in fig.II-3. The gas volume shape is a cylinder $5^{\prime \prime}$ in diameter and 17" Iong. The aluminum walls of the chamber are 3/32" thick. The ends of the cylinder were sealed with viton A "O" rings and 7" diameter, 2" thick pieces of Herculite plate glass*. Two Amperex XP1040 photomultipliers (PM's)view the gas scintillations through the glass plates.

*A tempered laminate of two 1" thick pieces, supplied by the Pittsburg Plate Glass Co. 


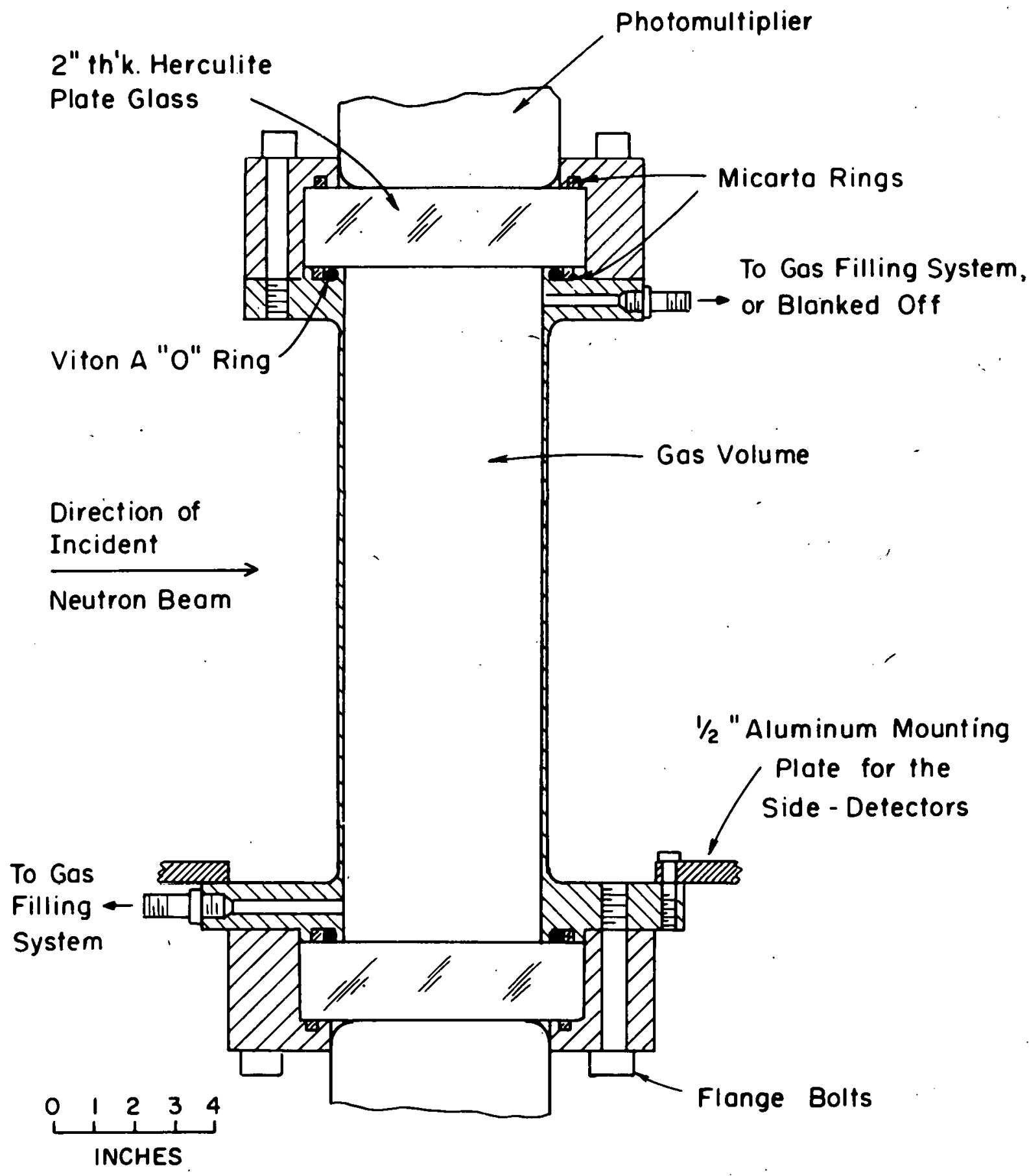

Aluminum Gas. Chamber 


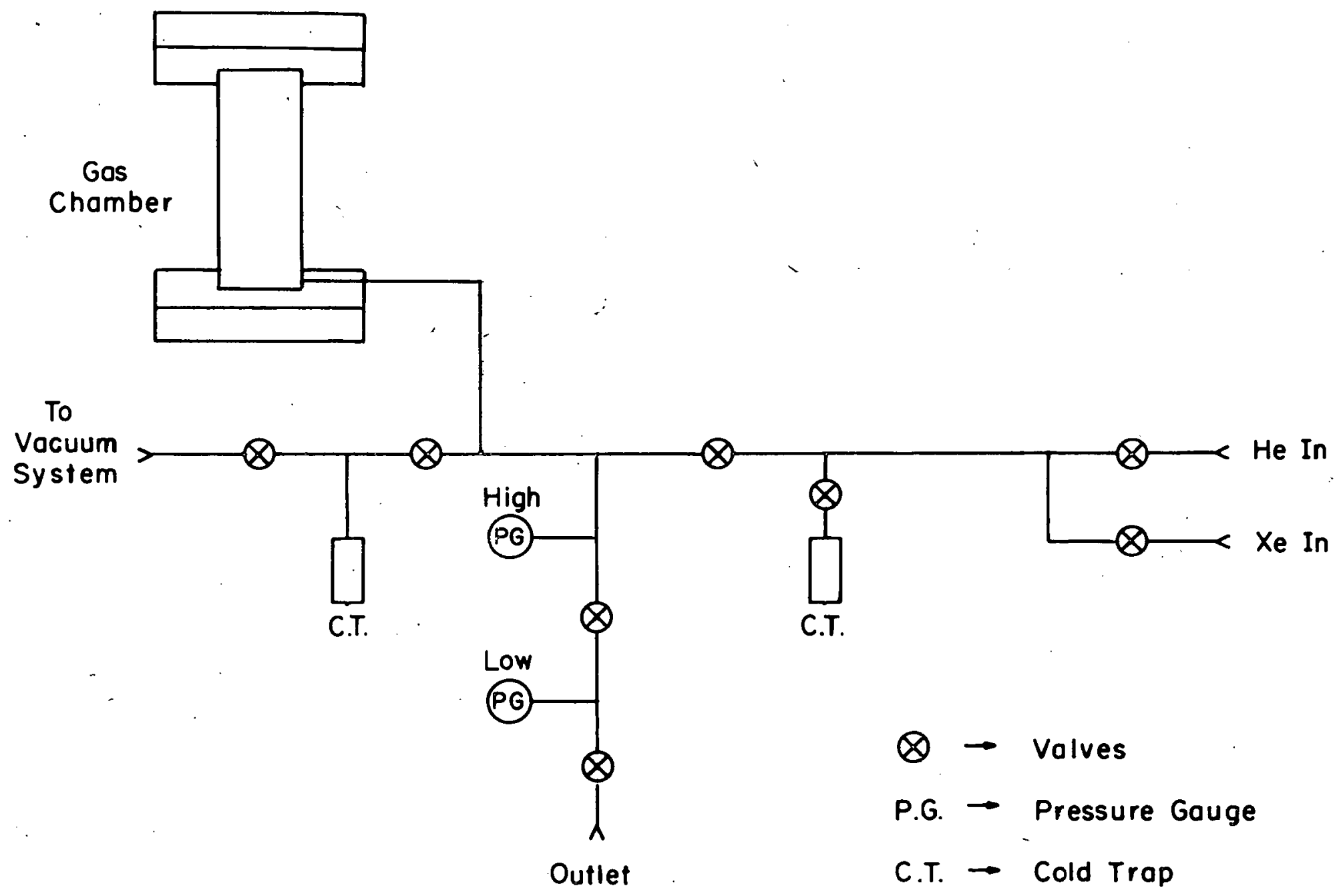

Gas Filling System for the Aluminum Gas Chamber

Fig. I I - 4 
The cylinder of the aluminum chamber was made long to reduce the scattering corrections introduced by the flanges holding the glass plates. The neutron beams incident on the gas chamber were generally about 6" in diameter, so the length of the cylindrical gas volume is about 3 times the beam diameter. Scattering corrections from the fianges arise from neutrons scattering first from the $\mathrm{He}$ and then from the flange material into the side-detectors. Also, the cylinder walls were made as thin as possible to further reduce scattering corrections. These corrections are discussed more fully in chapter VI.

The gas filling system for the aluminum chamber was a modification of the one used by S.Kowalski(46). An outline of the modified system is shown in fig. II-4. The system was constructed from $3 / 8^{\prime \prime}$ OD stainless steel pipe and fittings. The liquid nitrogen cold traps are used to freeze out the xenon which is added to increase the light output from the $\mathrm{He}^{4}$ recoils. A more complete description of the use of the filling system will be given in Section B-2.

The stainless steel chamber is basically that used by S. Kowalski(46), except that the cylinder walls have been machined to $1 / 8 "$, which is half the original thickness. The chamber was machined from type 304 stainless steel and is illustrated in fig. II-5. The gas volume is a cylinder 5" in diameter and 10" long. The ends are sealed with "O" rings and $7^{\prime \prime}$ diameter, $2 "$ thick pieces of Herculite plate glass. 


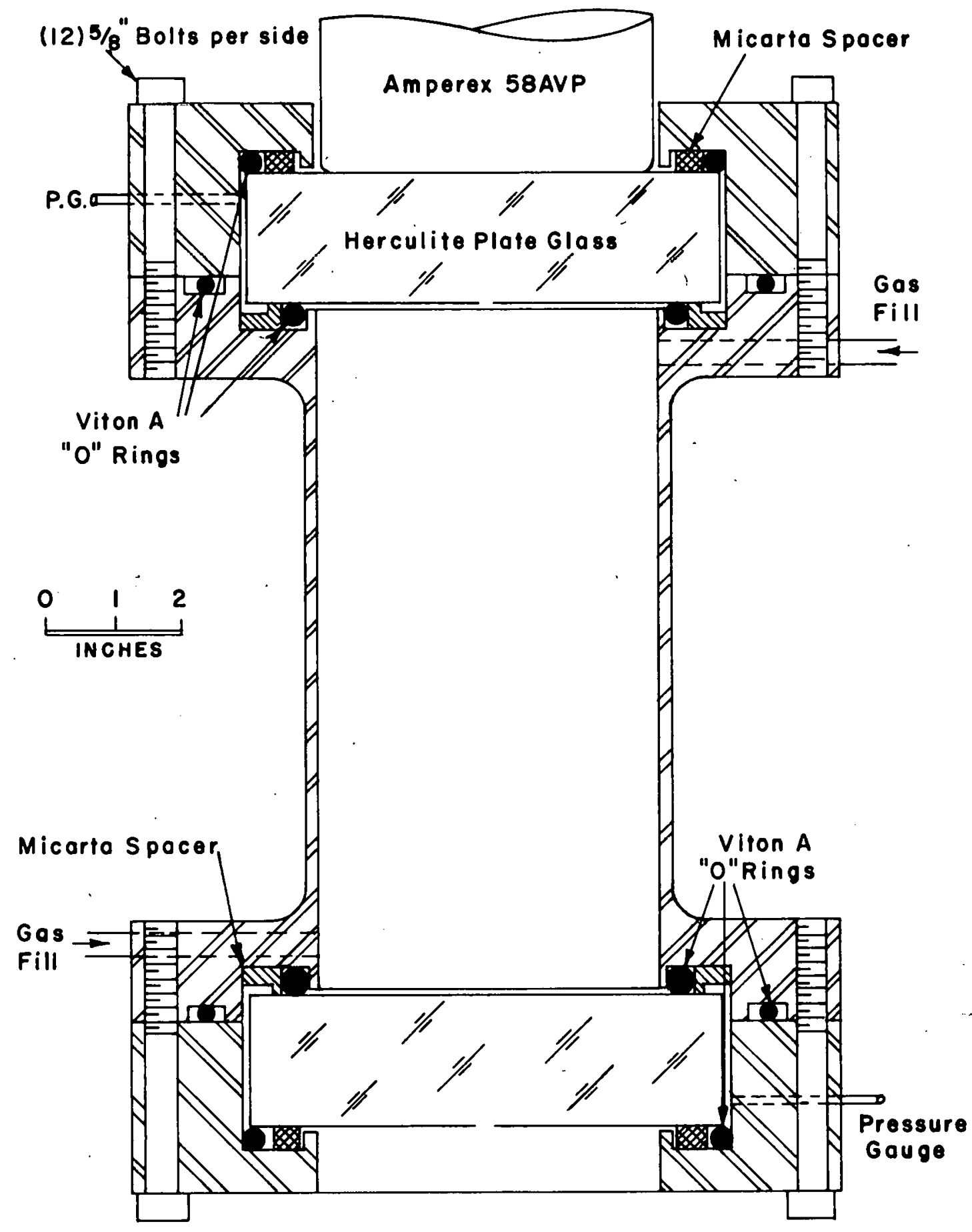

Stainless Stee1 Gas Chamber

Fig. I I -5 


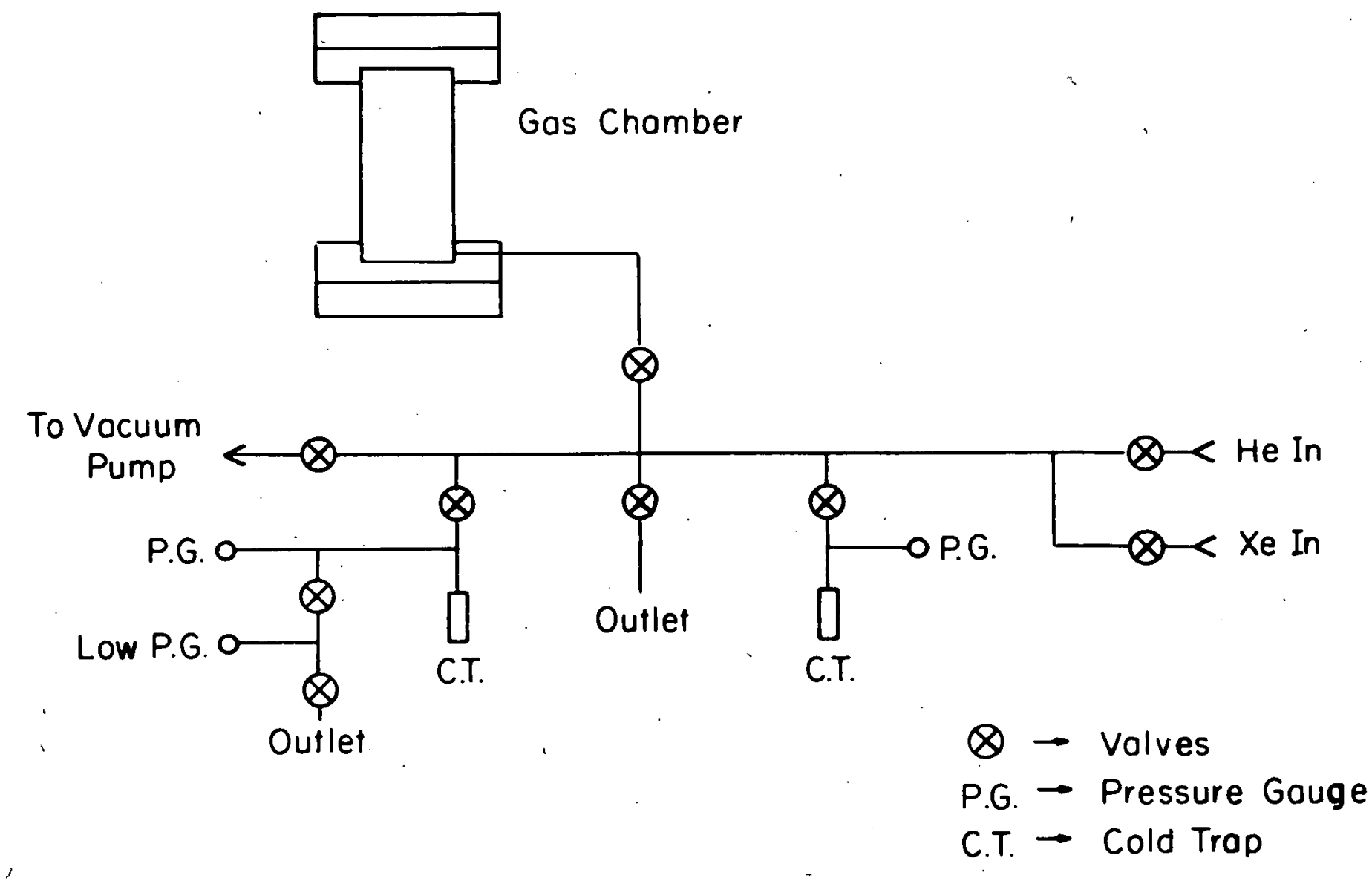

Gas Filling System for the Stainless, Steel-Gas Chamber

Fig. I I- 6 
When one of the valves on the aluminum chamber filling system developed a leak the system was completely rebuilt, and the new system is the one used with the stainless steel chamber. The new system uses Hoke HY441 high pressure bellows seal valves with teflon seats. The remainder of the system is $3 / 8$ " OD stainless steel pipe and fittings, plus the cold traps salvaged from the system used with the aluminum chamber. All joints were copper brazed in a hydrogen atmosphere, which also served to clean the system of oils and absorbed gases. The new system has a more convenient filling design, and is outlined in fig. II -6 .

2. Cleaning and filling the chambers and systems.

Since the amount of 1 ight output from the $\mathrm{He}^{4}$ recoils is strongly dependent on the purity of the He-Xe mixture $(47,48)$, great care was taken to make the chambers and systems as clean as possible. The final cleaning of the gas chambers was done after they had been hydrostatically pressure tested. They were wiped down thoroughly in a bath of first trichloroethylene, then methanol, and finally acetone, after which they were dried. This was done 2 or 3 times. For the heat treated aluminum chamber this was the only cleaning process used, since that chamber could not be heated above $100^{\circ} \mathrm{C}$ or it would lose its temper. The stainless steel chamber, however, was also fired in a hydrogen atmosphere retort at about $2000^{\circ} \mathrm{C}$, and this served to clean the chamber of occluded oils and most gases. The better cleaning of the stainless steel chamber was later demonstrated by a greater stability of light output from a gas filling in that chamber. 
The pipes and fittings for the filling systems were first cleaned with the three baths described previously, and then sent to another laboratory for chemical cleaning in preparation for brazing. Upon return the entire system was assembled and then sent out for copper brazing in a hydrogen retort. Therafter great care was taken to keep the inside of the systems as clean as possible.

With the gas chambers and systems very clean it was then necessary to obtain clean gases. The gas system had an oil diffusion pump and a rough pump connected to one outlet, and a tank of xenon and another of helium connected to two inlets. After some initial tests the diffusion pump was not used as greater cleaning of air from the system could be accomplished by other methods.

After the chamber had been opened to air it was generally cleaned by the following process. The rough pump evacuated the chamber to about 100 microns pressure and was then valved off. Helium was admitted to about $200 \mathrm{psi}$ and allowed to settle for a few minutes before being let out to atmospheric pressure. The system was then roughed down to 100 or less microns and the procedure repeated. After three or four such flushings with helium the chamber was quite free of atmospheric gases, as shown by the following observations. For the purposes of calibration (see section B-4) a Po 210 , alpha source was mounted in the gas chamber. After each helium filling this source could be observed as a broad peak (about $30 \%$ resolution) in the output of the gas-counter PM's. For the first 2 or 3 
helium fillings the initial pulse height of this peak would increase appreciably from one filling to the next, but for any given filling it would slowly decrease in time as the remaining atmospheric gases were redistributed and poisoned the helium seintillations. After about the fourth filling only slight increases (less than $5 \%$ ) in pulse height from one filling to the next were observed, and the pulse height for any given filling would remain relatively constant in time. This was used as an indication that the system and chamber were sufficiently clear of harmful gases.

Once the gas chamber and system were free of harmful gases the helium was rough pumped out and xenon admitted to about 80 psi absolute, which would give the proper amount of xenon for the final filling. The xenon was purified by placing liquid nitrogen around one of the cold traps to freeze out the xenon and then rough pumping on the xenon for about 15 minutes. This eliminated any harmful amounts of oxygen and nitrogen which may have been admitted with the xenon since their vapor pressures at the liquid nitrogen temperatures used are much greater than that of xenon. If the presence of water vapor was suspected the cold trap was warmed up sufficiently to vaporize the xenon, which was then refrozen in a second cold trap. After sealing the second cold trap, the first was heated up and pumped on to eliminate any water, which had remained frozen in the first trap.

The final filling was made in one of two ways. Usually the gas chamber was filled to about 80 psia of clean xenon and then helium added to bring the total pressure to 800 psi. With 
this method the gases were quite well mixed by the large flow of gas during the addition of the helium. The second method interchanged the order of gas filling, the cleaned xenon sealed off in a cold trap being added second. This method was used once to fill the stainless steel chamber and required several hours to achieve a uniform He-Xe mixture in the gas chamber. Since Xe comprises only $10 \%$ of a gas filling there was only a small flow of gas. into the chamber during the Xe addition and most of the Xe remained near the bottom where it had entered the chamber. This was shown by the varying light output for Po 210 alphas in different positions in the gas chamber. The Po ${ }^{210}$ calibration source consisted of three separate sources mounted about 2" apart on the gas chamber cylinder axis. During the early stages of gas mixing by the second method these sources could be quite well resolved because of the non-uniform gas mixture. To increase the rate of mixing for this method the walls of the gas chamber were gently heated to produce convective gas flow. For a given gas filling the light output from the Po 210 alphas proved sufficiently stable to allow the gas-counter to be useful in a polarimeter. With the aluminum chamber the pulse height of the Po ${ }^{210}$ alphas dropped to about $60 \%$ of its initial value in a period of two weeks. The pulse height leveled off near $50 \%$ of the initial value and thereafter continued to drop only a few percent a week. These numbers varied somewhat from filling to filling. 
The stainless steel chamber was cleaner than the aluminum one, having been fired in a hydrogen retort at $2000^{\circ} \mathrm{C}$, and this was shown in the better pulse height stability of the stainless steel chamber. With the stainless steel chamber the Po 210 alpha source pulse height decreased only about $10 \%$ a month, dropping about $50 \%$ in six months. This also shows that the reflective $\mathrm{MgO}$ and wavelength shifting coats on the cylinder wall (see section B-3), as well as the viton A "O"rings at the glass plates, are not serious poisoners of the gas scintillations.

\section{Light Collection}

When an energetic $\mathrm{He}^{4}$ nucleus is stopped in the high pressure He-Xe gas chamber its energy goes into ionizing and exciting the atoms in that gas, and some of the $\mathrm{He}^{4}$ nucleus ${ }^{\prime}$ energy ends up as light. The light output has been found to be a maximum for an approximately $90 \% \mathrm{He}-10 \%$ Xe combination (49), which is the composition used in the polarimeter-spectrometer. The object of the light collection system is to get a maximum amount of this light to the two 5" diameter XP1040 photomultipliers which view the gas scintillations through the 2" thick Herculite glass plates at each end of the gas chambers. Since the statistical variation of the photomultiplier output depends on the number of photons incident on the photocathode, good light collection is necessary to obtain good resolution of the $\mathrm{He}^{4}$ recoil energy.

An important element in obtaining good light collection is to make the walls of the gas chamber as reflective as possible. For this reason the chambers were fitted with $5^{\prime \prime}$ OD, $0.025^{\prime \prime}$ wall stainless steel sleeves which were coated with MgO. The 
MgO was mixed with cellulose powder and water and then painted onto the sleeves, where the mixture dried into a smooth, hard reflective coating. Use of the sleeves facilitated in making coating changes, and it was also much easier to work with the sleeves than with the heavy gas chambers.

Since most of the light from the Xe-He scintillations is in the ultra-violet which has a greatly reduced transmission through the $2 "$ thick glass plates and the photomultiplier faces, organic phosphors were used to absorb the ultra-violet and reemit in the visible region. For this purpose the MgO surface and the inside faces of the glass plates were coated with tetraphenylbutadiene (TPB). Diphenylstilbene (DPS) and $\mathrm{p}$-quaterphenyl ( $P Q P)$ were also tried. Tests with the gas chambers show that $\mathrm{TPB}$ is better than $\mathrm{PQP}$, giving about twice as much light for the Po 210 alphas. DPS has been found by some to be better than TP $(50)$ but TPB is more stable when exposed to water vapor in the air (51) was easier to vacuum evaporate, and thus was chosen for the polarimeter. TPB was used as the wavelength shifter for all the polarization data taken with the herein described polarimeter-spectrometer.

The MgO coated sleeves had a 100 to $150 \mathrm{ugm} / \mathrm{cm}^{2}$ layer of TPB vacuum evaporated over the $\mathrm{MgO}$. The glass plates had about $50 \mathrm{ugm} / \mathrm{cm}^{2}$ of TPB vacuum evaporated over the inner surface. Care had to be taken to match the coats on the two glass plates as otherwise there would be a large variation in light collection efficiency over the neutron-irradiated volume of the gas chamber. Matching was accomplished by selecting glass plates which gave comparable relative pulse heights 
for three po 210 sources separated by $2^{\prime \prime}$ on the gas chamber axis.

To form part of a good spectrometer the chambers must have uniform light collection over the central portion of the gas volume. The size of the neutron beams incident on the gas chambers cause the $\mathrm{He}^{4}$ recoils to occur uniformly throughout a 6" long central portion of the gas chamber, and any nonuniformity in light collection would decrease the ability to. resolve $\mathrm{He}^{4}$ recoils of different energy.

The method of measuring light collection uniformity was to compare the pulse height of two Po alpha sources in different parts of the gas chamber. It was necessary to open the gas chamber to air to move the Po sourses, and consequently the measurements on one source moved to various parts of the chamber would be made with gas fillings of differing purities. Since the pulse height for the Po alphas could vary by several percent for small impurities, measurements on one source could not test for $1-2 \%$ light collection uniformity. By using two sources, one located in a standard position, and comparing their pulse heights, 1 - 2\% variations in the relative pulse height, or light collection efficiency, could be measured.

These tests for light collection uniformity were made with gas fillings of about 50 psia of pure xenon. Xenon gives about $70 \%$ as much light output for the Ro 210 alphas as the $90 \% \mathrm{He}$ $10 \%$ Xe mixture so pulse height measurements accurate to $1-2 \%$ could be made.

Pure helium gives only about $1 / 5$ the 1 ight output of the optimum He-Xe mixture, resulting in about $30 \%$ resolution for 
the Po 210 alphas, and thus was not of use in measuring light collection uniformity.

Since the test procedure involved opening the gas chamber to air several times, pure xenon also had an advantage in that it could be frozen out in one of the cold traps, and thus one Xenon charge could be used for many fillings.

Po sources separated by about $2 "$ or more along the gas chamber axis were distinguished by using the anode output of one of the PM's which easily resolved the two sources. A single channel differential pulse height analyzer then had its window centered on the single PM anode peak of one of the sources, and drove in coincidence a multichannel pulse height analyzer which accepted the summed dynode-14 output of the two PM's. The dynode peaks obtained with the anode window centered on the peak of first one, then the other Po source were then compared. The limiting accuracy was about $1 \%$ in relative pulse height.

The results of the uniformity measurements give light collection as constant to within $2 \%$ for $\pm 2 "$ from the gas chamber center along the cylinder axis. At a given neight there is less than 1\% variation with distance from the axis, to within $1 / 4^{\prime \prime}$ of the wall. These tests were made only on the stainless steel chamber.

With properly matched glass plates the stainless steel chamber gave about $6 \%$ resolution for a single $5.3 \mathrm{MeV}$ Po 210 alpha source, and about $7 \%$ for three sources positioned on the cylinder axis, one in the center of the chamber and the other two at $\pm 2^{\prime \prime}$ along the axis. For similar conditions the 
aluminum chamber gave about $15 \%$ resolution, both for a single and for three sources.

4. Calibration of $\mathrm{He}^{4}$ recoil energy vs. PM pulse height. To measure the energy of a neutron scattered by the $\mathrm{He}$ it is necessary to convert the photomultiplier output for a $\mathrm{He}^{4}$ recoil into a corresponding energy. The $\mathrm{He}^{4}$ recoil is calibrated in energy by comparing the $\mathrm{He}^{4}$ pulse height with that for the $5.3 \mathrm{MeV}$ alphas of a Po 210 source. The Po 210 calibration is made with three sources separated by $2^{\prime \prime}$ on the gas chamber cylinder axis near the geometrical center of the gas chamber. The sources consist of $3^{\prime \prime} 1$ engths of $10 \mathrm{mil}$ dimaeter silver wire with $1 / 4^{\prime \prime}$ of one end coated with polonium.

The use of three axial sources made it easier to match the gains of the two PM's, particularly when the middle source was not precisely in the geometrical center of the gas chamber, and also weighted the calibration over a larger portion of the neutron-irradiated gas volume.

The calibration of the gas-counter is specified by two numbers, $\mathrm{C}_{\alpha}$ and $\mathrm{C}_{\alpha / 2} \cdot \mathrm{C}_{\alpha}$ corresponds to the pulse height of the Po-alphas and is the channel of a multichannel pulse height analyzer at which the peak for the Po-alphas falls. $C_{\alpha / 2}$ is the channel corresponding to the Po-alphas when the summed output of the PM's is attenuated by a factor of two. $\mathrm{C}_{\alpha}$ is the channel for a $5.3 \mathrm{MeV}$ Po-alpha, and $\mathrm{C}_{\alpha / 2}$ is taken as the channel for a 2.65 MeV alpha. The channel vs. alpha energy points so specified are referred to as the Po-alpha calibration points.

A check of the Po-alpha calibration was made with 14.7 $\mathrm{MeV}$ neutrons from the $\mathrm{t}(\mathrm{d}, \mathrm{n})$ reaction. For this check the 
$150 \mathrm{kv}$ Cockcroft-Walton accelerator of the M.I.T. Nuclear Engineering Dept. was used. From fig.II-7, which shows the experimental arrangement, one can see that the neutron beam irradiating the gas-counter is geometrically very nearly identical to that used during the photodisintegration runs (see fig.III-D). Side detectors were placed around the gas chamber at a number of neutron scattering angles. The peaks in the $\mathrm{He}^{4}$ recoil spectra then give channels corresponding to a specified set of $\mathrm{He}^{4}$ recoil energies, which are calculated from the neutron scattering angles of the side-detectors by. using eq. II. 8 .

The results of the calibration check are plotted in fig. II-8. The graph shows that pulse height (channeI) has a linear relationship to the $\mathrm{He}^{4}$ recoil energy, and that the Po-alpha calibration points are about $10 \%$ too low in channel number for true 5.3 and $2.65 \mathrm{MeV} \mathrm{He}^{4}$ recoils, which can be produced by scattering the $14.7 \mathrm{MeV}$ neutrons at the appropriate angles. The channel corresponding to zero pulse height is effectively the same for both the Po-alpha calibration and the $\mathrm{He}^{4}$ recoil calibration. Nevertheless, the Po alpha sources were used during actual experiments because of their great convenience.

The difference in the two calibrations requires that the Po-alpha calibration be corrected. As will be shown in chapter IV, the analysis of polarization data requires each run to have two calibration points, $\mathrm{C}_{\alpha}^{\prime}$ and $\mathrm{C}_{\alpha / 2}^{\prime}$, where $\mathrm{C}_{\alpha}^{\prime}$ is the analyzer channel corresponding to a true $5.3 \mathrm{MeV} \mathrm{He}^{4}$ recoil and $\mathrm{C}_{\alpha / 2}^{\prime}$ is the channel for a $2.65 \mathrm{MeV} \mathrm{He}^{4}$ recoil, which recoils are produced 


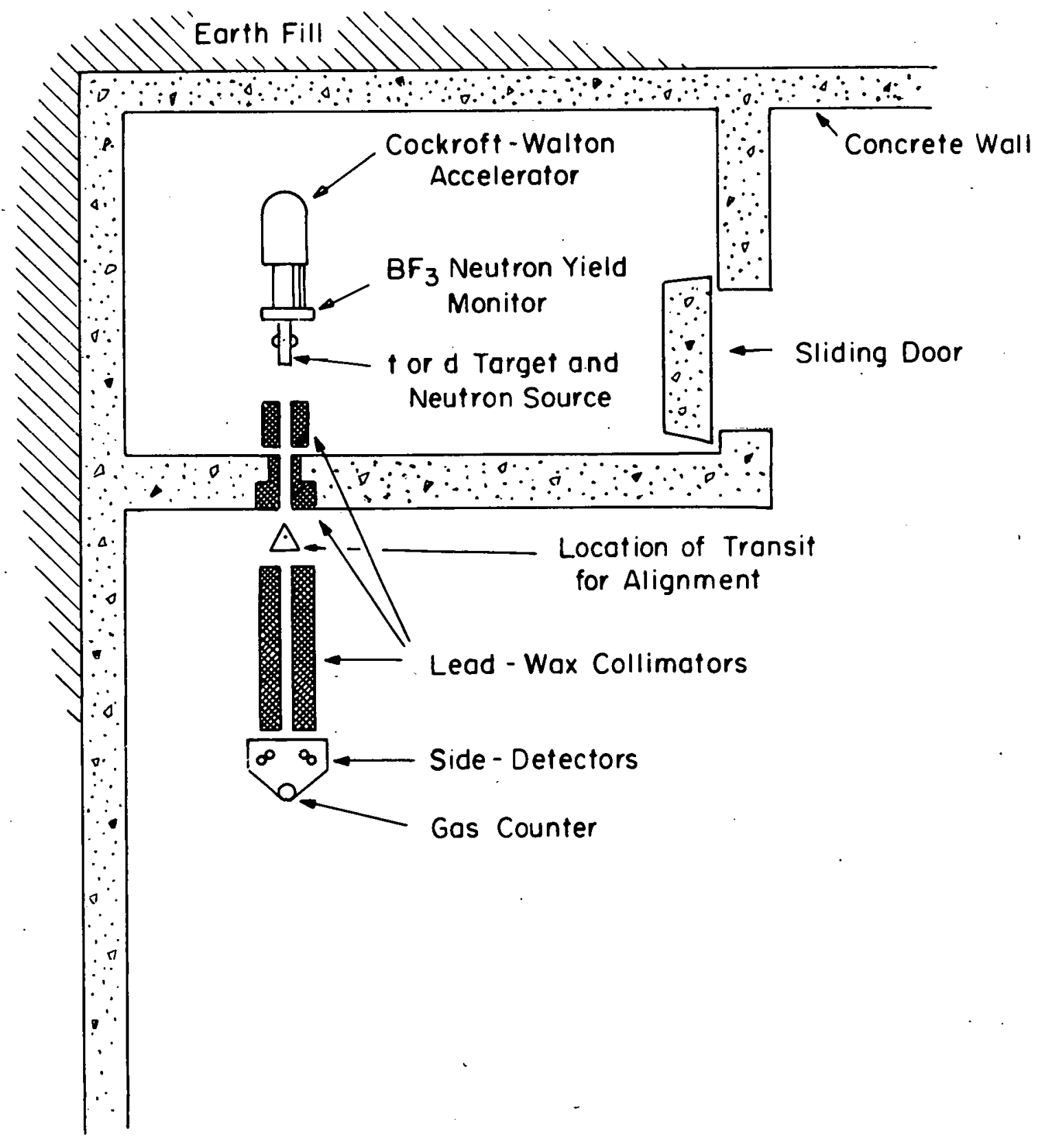

Layout for Irradiating the Polarimeter with $14.7 \mathrm{MeV}$ Neutrons

Fig. II -7 


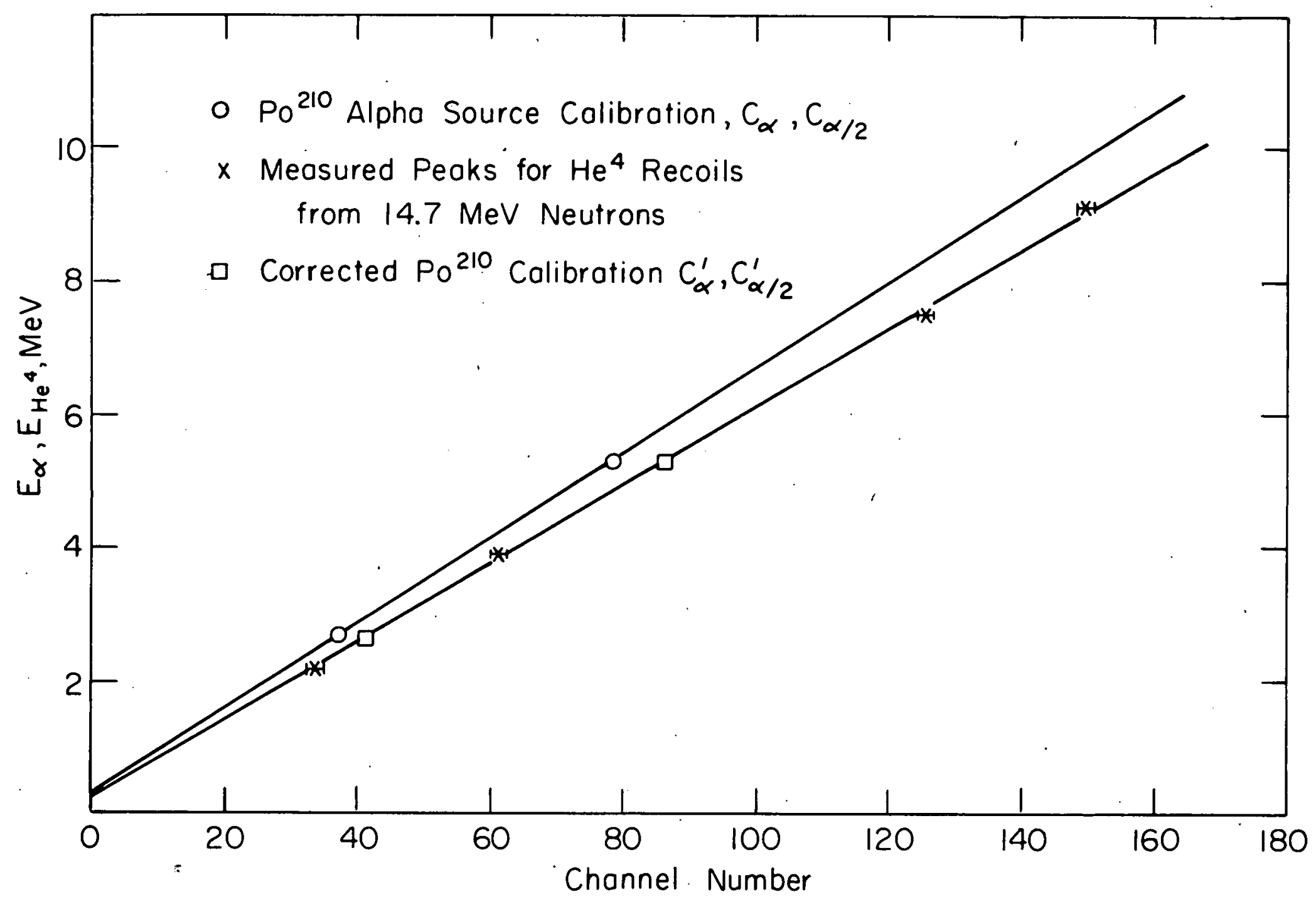

Plots of Po ${ }^{210}$ alpha Calibration and of the $\mathrm{He}^{4}$ Recoil Calibration'

Fig. II -8 
by scattering a neutron of energy $E_{n}$ at an appropriate angle $\theta_{0}$. Since the channel for zero energy is the same for both calibrations, the corrections can be made of the form

$$
\begin{aligned}
& C^{\prime} \alpha=C_{\alpha}+2 r\left(C_{\alpha}-C_{\alpha / 2}\right) \\
& C^{\prime}{ }_{\alpha / 2}=C_{\alpha / 2}+r\left(C_{\alpha}-C_{\alpha / 2}\right)
\end{aligned}
$$

where

$$
r=\frac{\left(\frac{\left(\frac{\Delta \text { channel })}{\Delta M e V}\right)}{\left(\frac{\Delta \text { channel })}{\Delta M e V}\right)} \text { Po - alpha calibration } \mathrm{He}^{4} \text { recoil calibration }\right)}{\left(\frac{1}{\Delta \mathrm{MV}}\right)}-1
$$

If we set

$$
\begin{aligned}
\mathrm{C}_{0}=2 \mathrm{C} \mathrm{a}_{2}-\mathrm{C}_{\alpha}= & \text { channel corresponding to zero pulse } \\
& \text { height } \\
\mathrm{C}_{\exp }= & \text { experimental measurement of the } \\
& \text { channel for a } \mathrm{He}^{4} \text { recoil of energy } \\
& \mathrm{E}_{\text {exp (obtained by scattering a neutron }} \\
& \text { of energy } \left.\mathrm{E}_{\mathrm{n}} \text { at an angle } \theta_{0}\right)
\end{aligned}
$$

then the calculated channel for the recoil, using the Poalpha calibration, is

$$
C_{\text {calc }}=C_{0}+\frac{E_{\exp }}{5.3}\left(C_{\alpha}-C_{0}\right)
$$

and this gives

$$
r=\frac{C_{\exp }-C_{c a 1 c}}{C_{c a l c}-C_{o}}
$$

The value of $r$ was measured for several He-Xe mixtures. Fig. II-9 shows the results plotted as $r$ vs. $P_{H e}$, the absolute helium partial pressure in the gas chamber, in psi. Fig. II-10 shows the results plotted as $\mathrm{r}$ vs. $\mathrm{P}_{\mathrm{Xe}}$, the absolute xenon 


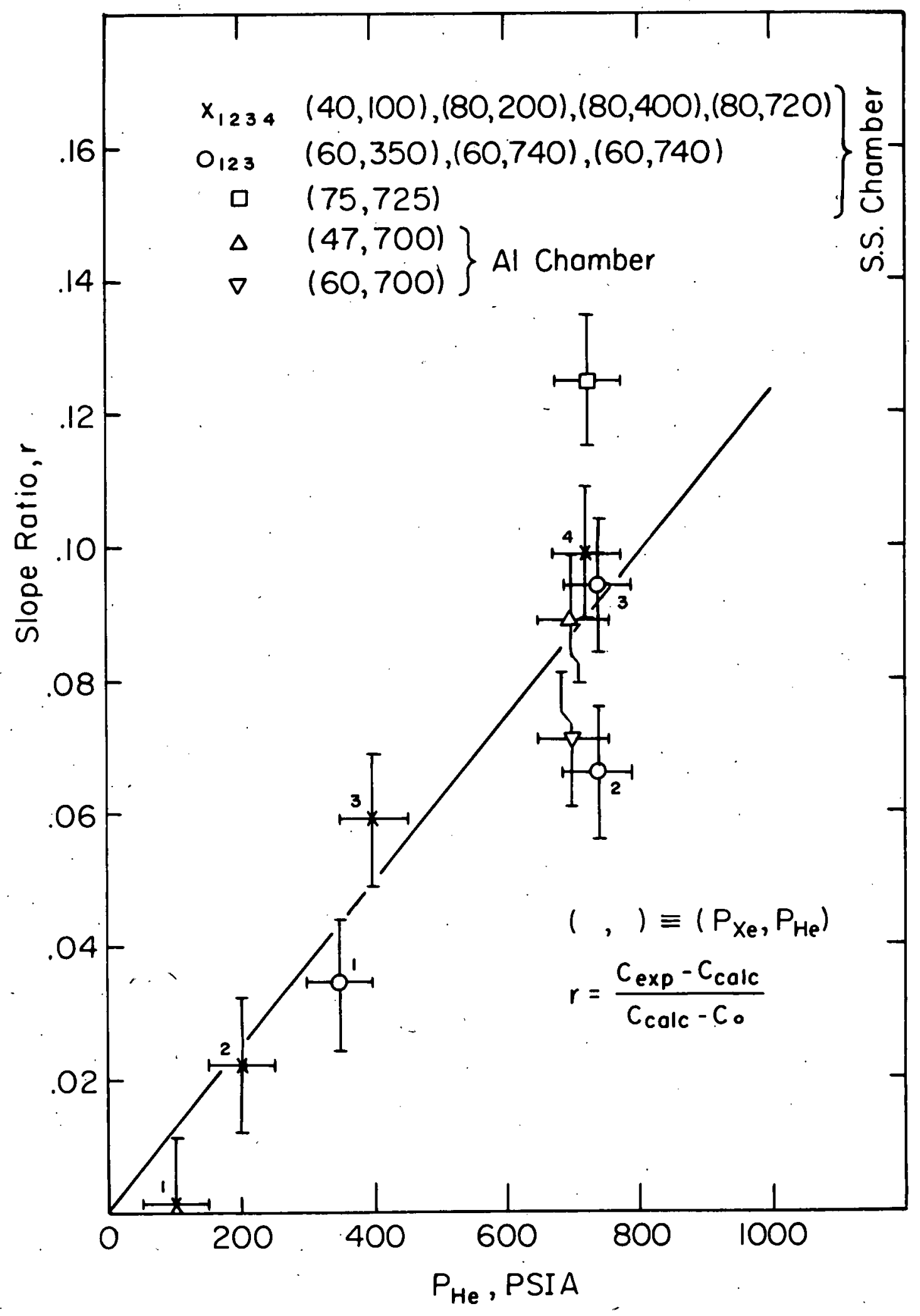

Plot of Slope Ratio vs. Helium Pressure

Fig. I I - 9 


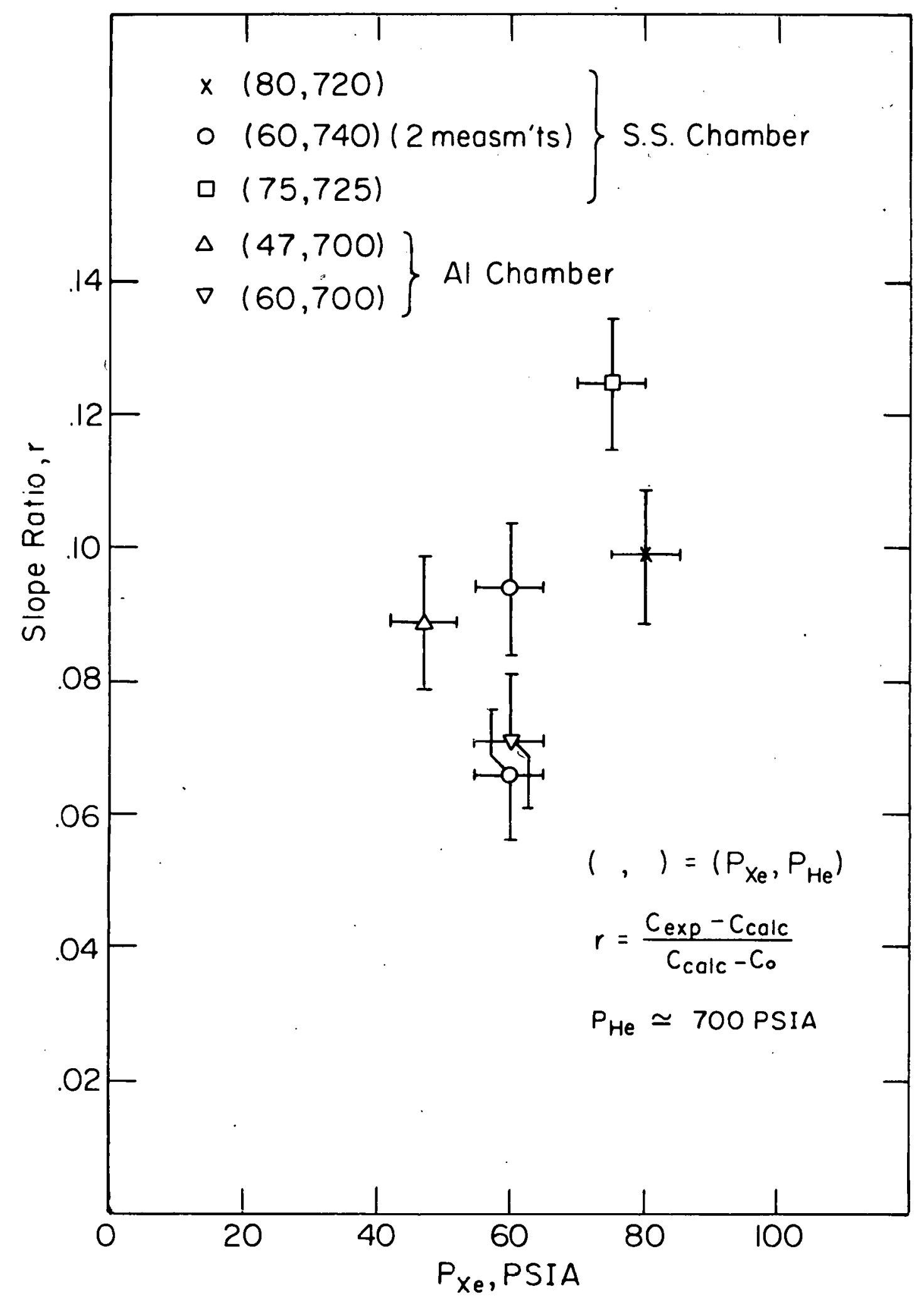

Plot of Slope Ratio vs. Xenon Pressure

Fig. II -10 
partial pressure, with $\mathrm{P}_{\mathrm{He}} \approx 700$ psia.

The results in figs. II-9 and II-10 indicate that the correction can be approximated by.

$$
r \simeq 0.082\left(\frac{\mathrm{P}_{\mathrm{He}}}{700}\right)
$$

The origin of the correction $r$ is not well understood. It could arise from the absorption of light by the Po alpha sources whose diameter is $0.025 \mathrm{~cm}$. This could be a significant offect, as the range of the $5.3 \mathrm{MeV}$ Po alphas is only about $0.16 \mathrm{~cm}$. for 60 psia of xenon and 700 psia of He: However, this can not explain the entire effect as the dependence on He pressure is too strong, (most of the stopping power is due to the $\left.\mathrm{Xe}_{.}\right)$. Perhaps some spectral changes are occurring as a function of He pressure, affecting the amount of absorption by the sources.

The correction given by Eq. II.20 is estimated to be accurate to $10 \%$ of its value, and since the correction was usually about $10 \%$ this gives the overall uncertainty in the corrected calibration as about $1 \%$. This is well with the resolution of the gas-counters, and the variations coming from non-uniform light collection and drifts in the gain of the electronics. Consequently eq. II.20 is sufficiently accurate to correct the gas-counter calibration for high pressure He-Xe mixtures, with

$$
\mathrm{P}_{\mathrm{He}} \simeq 700 \text { psia, and } \mathrm{P}_{\mathrm{Xe}} \simeq 60 \text { psia. }
$$

Eq. II.20 was only used to correct the Po-alpha calibration for polarization runs made with the aluminum gas chamber. The resolution of the stainless steel gas chamber was sufficient 
to resolve some of the peaks in the $0^{16}(r, n)$ spectrum, which peaks have known energies from time-of-flight measurements. These measured peaks were used to calibrate the energy of the $\mathrm{He}^{4}$ recoils for the data taken with the stainless steel chamber. C. The $\mathrm{He}^{4}$-scattered-neutron detectors (the side-detectors).

\section{Description}

The side-detectors used to detect the neutrons scattered from $\mathrm{He}^{4}$ in the He-Xe gas scintillation counter are illustrated in fig. II- 11. They are constructed from stainless stee1 pots $6 "$ in diameter and $7 "$ high. The scintillator used was analytical grade toluene activated with 4 gms/liter of $\mathrm{p}$-terphenyl and 0.2 gm/liter of POPOP. Light collection was improved by coating the inside surface of the pots with $\mathrm{MgO}$ in a binder of sodium silicate.

The fast (about 7 ns rise time) scintillations from recoil protons in the toluene were viewed by two RCA $6810 \mathrm{~A}$ photomultipliers mounted on top of the pots and with their faces in the toluene: The PM's and the pots were wrapped with u-metal to provide magnetic shielding.

To reduce the counting rate from gamma rays the sidedetectors were wrapped with a 1/4" thick sheet of lead. The gamma rays arise mainly from the Compton scattering of bremsstrahlung photons, first in the photoneutron target and then by the gas chamber cylinder walls, and from the inelastic scattering of neutrons by the metal gas chamber.

For polarization measurements eight side-detectors were used, forming four pairs for asymmetry measurements. With the aluminum gas chamber the four pairs were mounted at a 


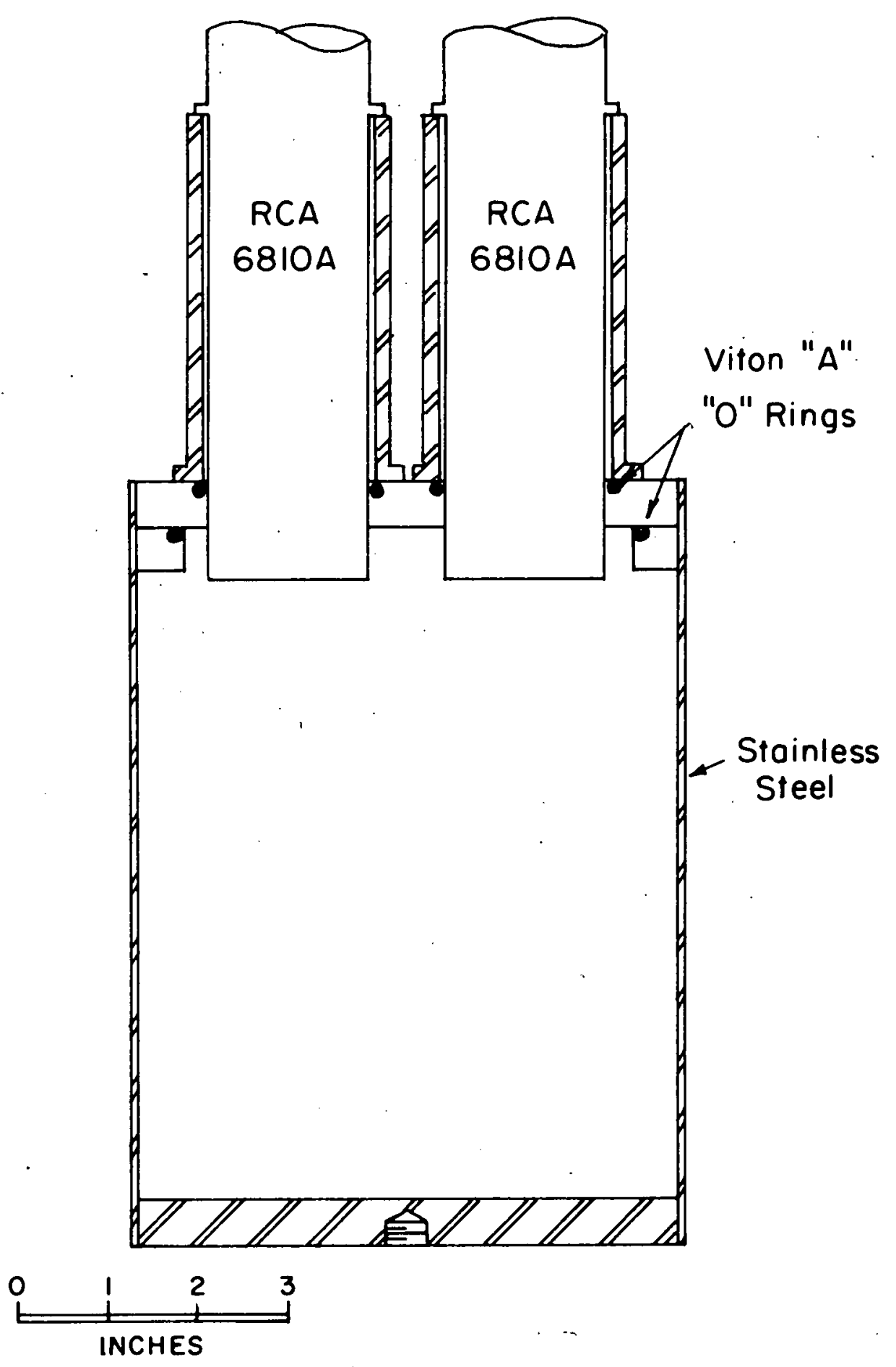

Proton Recoil Neutron Detectors

Fig. II-11 
few combinations of $47,67,88,117$ and 137 degrees, these being the center-to-center laboratory neutron scattering angles. The side-detectors were $20^{\prime \prime}$ from the aluminum chamber (center to center), and were mounted with the aid of accurately located holes in a 1/2" thick aluminum plate. The layout is shown in fig. II-12. Drilled and tapped holes in the center of the side-detector bases were used to bolt the detectors in the proper position on the aluminum plate. The aluminum plate was mounted to the base of the aluminum gas chamber by means of $1 / 4$ " steel dowel pins in accurately machined matching holes. Machining accuracy was $\pm 0.005^{\prime \prime}$ and this gives an accuracy in the position of the side-detectors of \pm 1 minute of arc in the central scattering angle.

A different aluminum plate for mounting the side-detectors was used with the stainless steel gas chamber. The lab angles were $113,127,141$, and 155 degrees, al1 30" from the gas chamber. For photoneutron polarization measurements the sidedetectors were mounted in the plane containing $\vec{k}_{\gamma}$ and $\vec{k}_{n}$.

2. Monitoring photomultiplier gains and setting detection thresholds.

Each side-detector had a Po 210 alpha source mounted in the toluene. The summed dynode-14 output of the PM's was pulse height analyzed and the gain of the PM's measured by finding the peak for the $5.3 \mathrm{MeV}$ alphas. Using this method of gain measurement the two PM's were adjusted to have equal gain by varying their high voltages. 


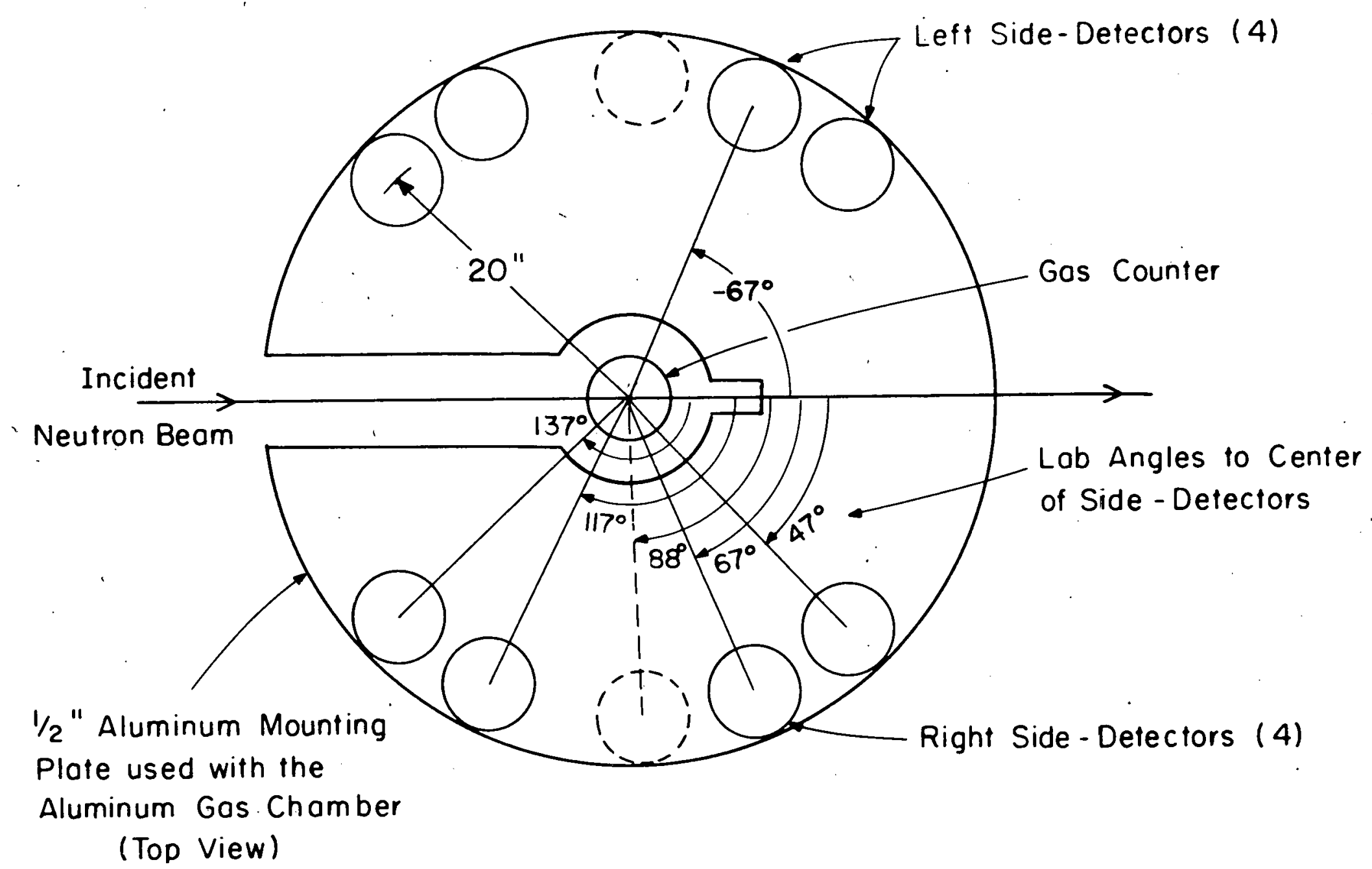

Mounting Plate for Side-detectors Used with the Aluminum Gas Chamber

Fig. II -12 
The anode output of the two PM's of a given sidedetector were summed, amplitude limited; and then clipped to provide time-width limiting. The resulting limited and clipped pulses were fed to a pulse shaper whose triggering level, together with the PM gains, set the threshold for the side-detector. To determine the threshold of a side-detector the dynode-14 pulse height spectrum of the alpha source was measured in coincidence with the anode pulse shaper output. This gave a spectrum of the $5.3 \mathrm{MeV}$ alphas with a low energy cut-off corresponding to the anode pulse shaper threshold. The cut-off was typically 4 channels wide ( $10 \%$ to $90 \%$ ) with the alpha peak in channel 40 (see fig. II-13). Using the known alpha to proton energy ratio for equal pulse heights, this cut-off was a measure of the neutron detection thresholds of the side-detectors. The neutron thresholds were then set by adjusting the photomultiplier gains and the anode pulse shaper thresholds.

When making neutron polarization measurements it is necessary that the detection efficiency of the side-detectors not change when the right and left members of a pair are interchanged. Since the detection efficiency is set by the PM gain and the anode pulse shaper threshold these were measured periodically to check their stability. As the dynode-14 output was otherwise unused, this dynode output was pulse height analyzed, and by measuring the channel of the alpha peak it was possible to monitor the PM gains during experiments. In this way the PM gains were checked before and after interchanging the members of a side-detector pair. The anode 


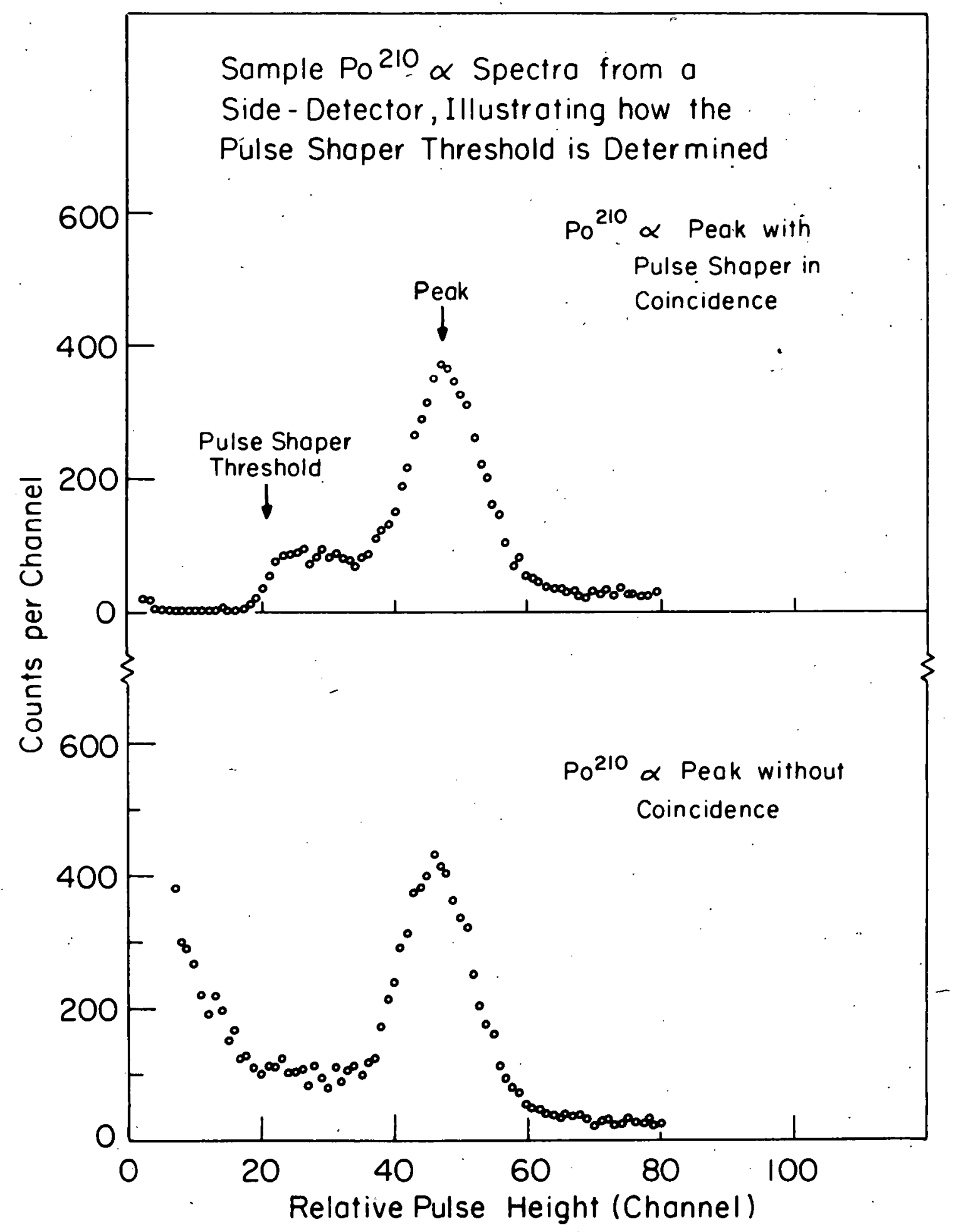

Po 210 Spectrum in a Side-detector, Illustrating the Threshold Fig. II-13 
pulse shaper thresholds were checked by measuring their threshold for pulses from a precision pulse generator. The absence of change in the alpha peak and in the anode pulse shaper threshold was taken to indicate a stable neutron detection efficiency.

3. Setting the gas-counter to side-detector coincidence timing.

In section A-4 it was stated that the gas-counter and side-detector must be triggered in coincidence, making allowance for the neutron flight times. The minimum flight time, $\tau_{\text {min }}$, was determined by the angle of the $\mathrm{He}^{4}(\mathrm{n}, \mathrm{n})$ scattering, the highest energy neutron of interest, and the gas-counter to side-detector distance. The maximum flight time, 'max, was determined by the lowest energy neutron of interest. The difference of these two flight times, $\tau_{\text {res }}={ }_{\max }{ }^{-}{ }_{\text {min }}$, is the total resolving time required by the coincidence circuit.

At this point it is necessary to describe the portion of the electronics which generates the gas-counter to sidedetector coincidence pulses. The anode outputs of the gast counter PM's and of the side-detector PM's both trigger pulse shapers which put out standard amplitude rectangular pulses, whose width is one half of the required resolving time, $\tau$ ps $=$ ${ }^{T}$ res/2. These pulses then go to a coincidence circuit which generates a standard output pulse for any input pulse overlap (see fig. II-14). The coincidence timing can be changed by adding to or taking out cable from the line traveled by the gas-counter or side-detector anode pulses. In practice this 
(A) Gos-Counter and Side-Detector PM's

Pulsed Simultaneously, Timing

Properly Set.

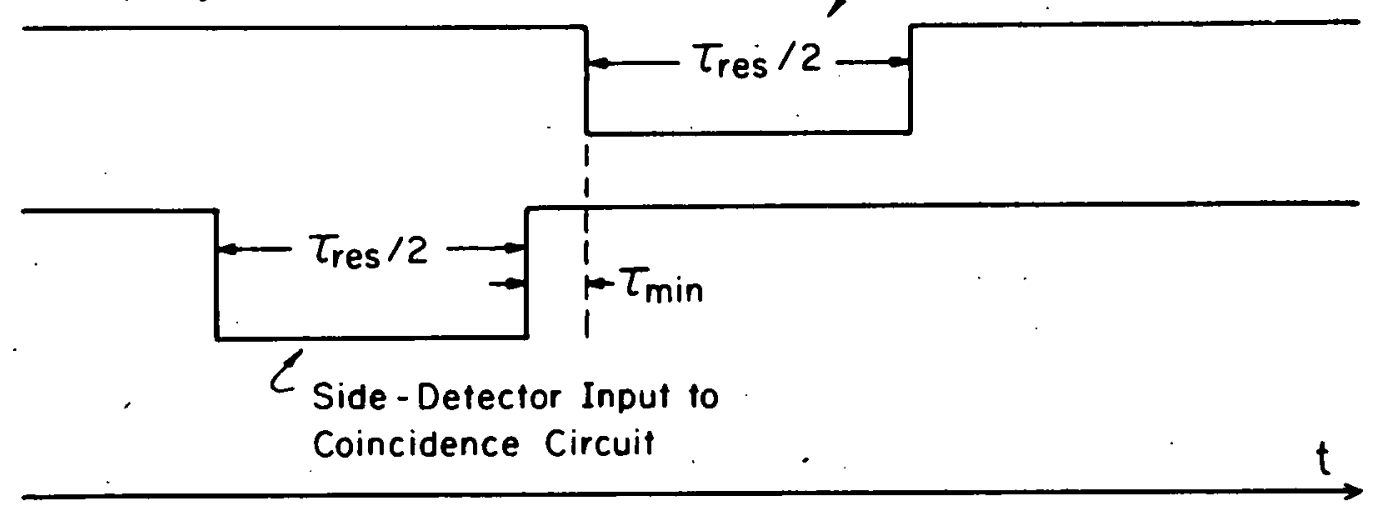

(B) Side-Detector Pulsed a Time $\tau_{\min }$ Later than the Gos-Counter

G - C input.

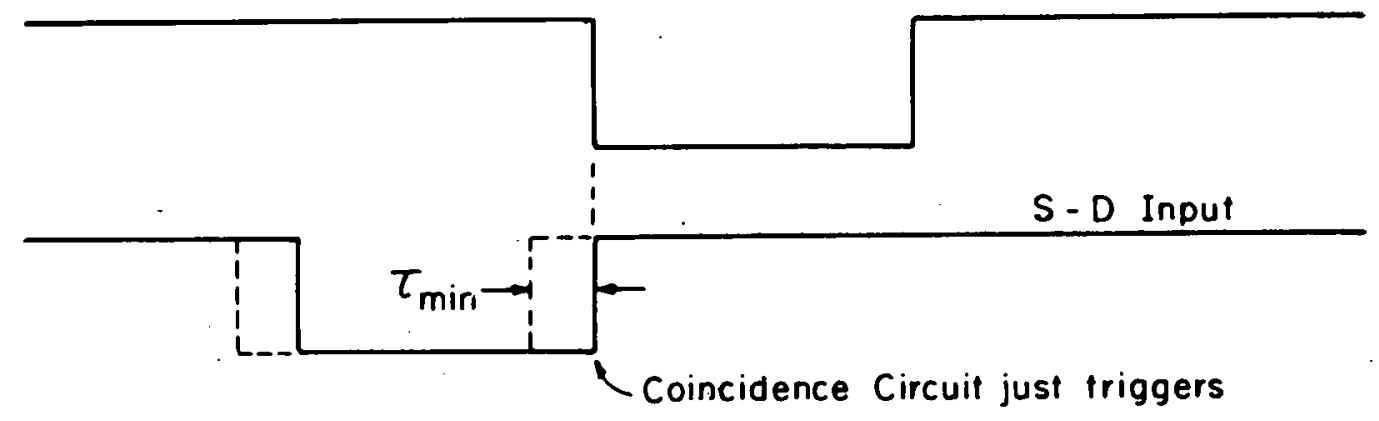

(C) Side-Detector Pulsed o Time $\tau_{\max }$

Loter thon the Gos-Counter

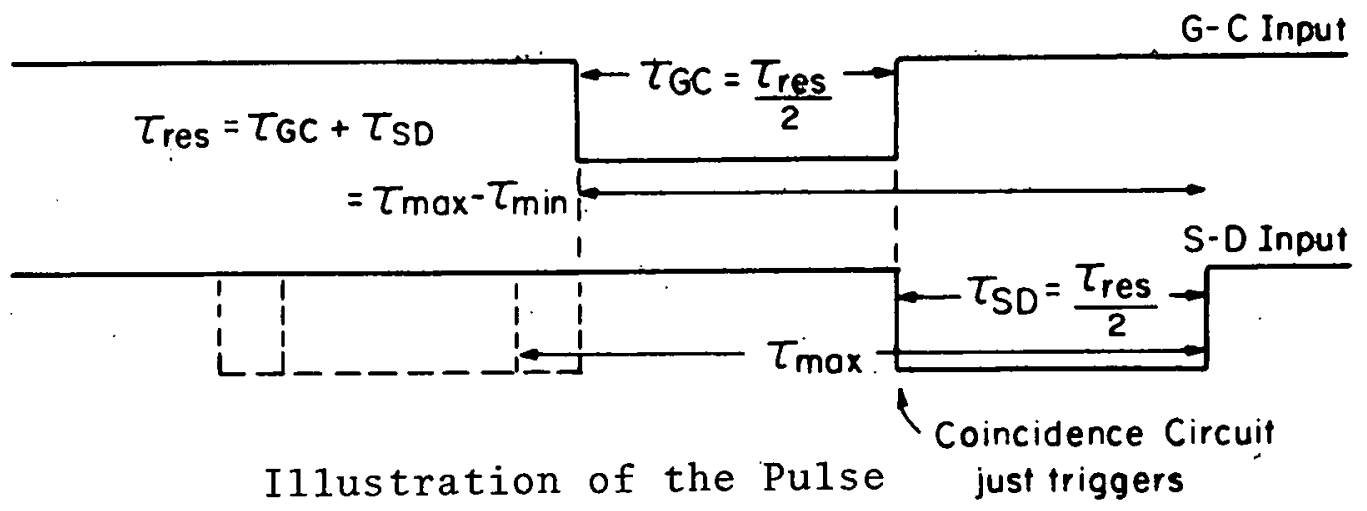

Relationship at the Input to the Coincidence Circuits

Fig. II -14 
timing adjustment was made in the cable connecting the side-detector PM's to their pulse shaper.

The gas-counter to side-detector timing was measured by triggering the PM's of the gas-counter and of a side-detector simultaneously, and observing the inputs to the coincidence circuit with an oscilloscope. The PM's were triggered with small fast flash tubes (52) and a sampling oscilloscope was usied to observe the coincidence circuit inputs. Fig. II-14 shows how the pulses appear and how the timing is measured. The timing is set so that the side-detector pulse ends a time $\tau_{\text {min. }}$. before the gas-counter pulse starts, as seen at the coincidence circuit input. This would give coincidence pulses for scattered neutron flight times of $\tau_{\min }$ to ${ }^{\tau} \min ^{+\tau} \operatorname{res}=\tau_{\max }$. Typical values of these times are $\tau_{\text {inin }}=7 \mathrm{~ns}, \tau_{\text {res }}=60 \mathrm{~ns}$, $\tau_{\max }=67 \mathrm{~ns}$, for a side-detector at 155 degrees, $30 "$ from the gas chamber.

The timing for a given side-detector is not a sharp. function, of neutron energy. There are variations in PM pulse height which cause triggering time jitter in the pulse shapers. Differences in neutron flight time because of the nonzero dimensions: of the gas-counter and side-detector also add some imprecision to the timing. For these reasons a safety factor generally was used to make the timing several ns broader than for the ideal limits.

D. Electronic Logic

The electronic logic used to carry out the operations of the $\mathrm{He}^{4}$ polarimeter-spectrometer will now be described. A block diagram of the circuit logic is shown in fig. II-15, 


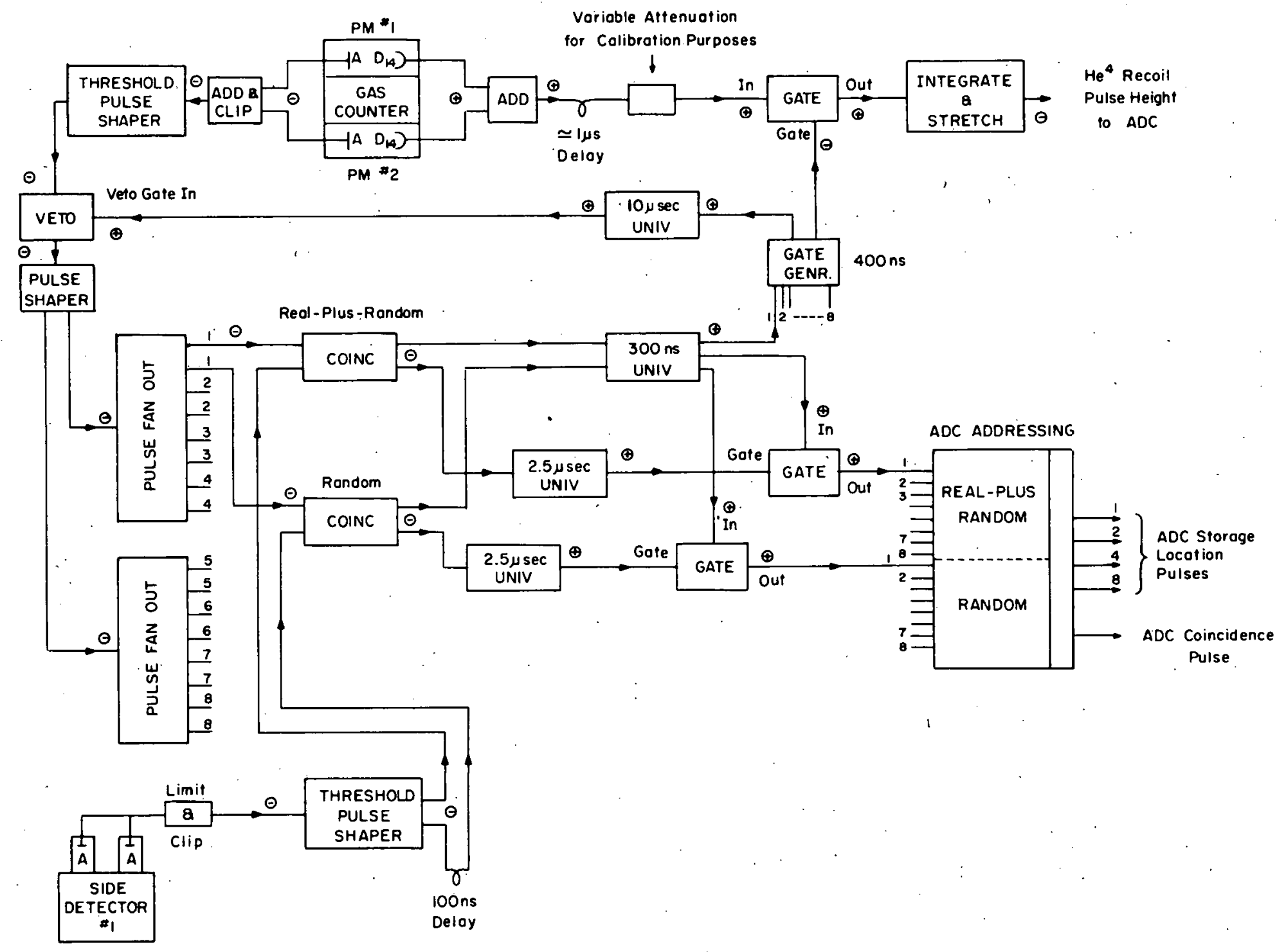

Polarimeter-Spectrometer Electronic Circuit Logic

Fig. II -15 
where the detailed logic is given for only one side-detector, the other 7 being identical. Circuit diagrams of the individual components are given in Appendix I .

The anode output of the gas counter PM's was used to set the $\mathrm{He}^{4}$ recoil detection threshold, and for timing with the side-detector pulses. The added and clipped anode output drove a threshold-setting pulse shaper whose output passed through a veto circuit which "turned off" the gas-counter when an event was being analyzed. The output from the veto circuit drove a pulse shaper which in turn drove two pulse fan-outs to provide 16 timing pulses for one input of each of 16 coincidence circuits.

The clipped and limited anode output of each sidedetector drove a threshold-setting pulse shaper, of which one output went to the real-plus-random coincidence circuit, and the other output went to the random coincidence circuit but with a delay of about 100 ns. The side-detector input to the real-plus-random coincidence circuit had its timing w.r.t. the gas-counter pulse set as described in section $\mathrm{C}-3$.

The triggering of a coincidence circuit indicated that an event was to be analyzed and the output of the coincidence circuit was required to perform three functions. First; signals were generated to specify which side-detector had. triggered a coincidence circuit, and whether the coincidence was real-plus-random or random. Second, a veto pulse was generated to block gas-counter anode pulses and prevent a second coincidence from interfering with the analysis of the first. And last, a gate pulse was generated that allowed the 
properly delayed gas-counter dynode pulse to be integrated and stretched. The integrated and stretched dynode pulse is proportional to the $\mathrm{He}^{4}$ recoil energy, and went to an anaiogue to digital converter (ADC) for pulse height analysis.

The gas-counter dynode pulses were gated to prevent pileup of the integrated and stretched pulses. The gate for the dynode pulses was 400 ns wide, which allowed the entire pulse to be integrated, and covered the about $30 \mathrm{~ns}$ spread in the relative positions of the gate pulse and the dynode pulse, which arises because of different neutron flight times.

During actual photoneutron polarization measurements. the neutron beam was produced in bursts about 4.5 usec wide, so the $A D C$ was required to accept pulses only during the times the neutron beam was irradiating the polarimeter. This helped reduce the background of neutrons from cosmic rays and natural radioactivity.

The gas-counter was calibrated with the Po 210 alpha sources in the He-Xe mixture by connecting two outputs from the fanout circuits to one coincidence circuit. This resulted in a gate pulse to allow the integration and stretching of all gas-counter pulses, and provided a coincidence pulse for the ADC to permit analysis of the dynode pulse. The analyzed dynode pulses gave a peak for the Po alphas, $C_{\alpha}$, which is one of the calibration channels introduced in section B-4. The second calibration channel, $\mathrm{C}_{\alpha / 2}$, was obtained in the same way, but with the dynode pulses attenuated by a factor of 2 just before the gate. 


\section{CHAPTER II I \\ EXPERIMENTAL PROCEDURE}

\section{A. Physical Layout.}

Fig. III-1 shows the physical layout of the experiment, which was performed at the RPI Linear Accelerator in Troy, New York. Electrons from the linac produced bremsstrahlung which were collimated in the forward direction and illuminated the photoneutron targets. Photoneutrons from the targets were collimated and illuminated the polarimeter, which was set up in an $8^{\prime}$ diameter tunnel. The polarimeter was shielded from the target room by an $8^{\prime}$ thick wall of heavy concrete, through which the collimator passed, and by earth fill surrounding the tunnel.

The electron beams for bremsstrahlung production consisted of bursts 4.5 usec wide with an average current in the bursts of 200 to $800 \mathrm{ma}$. The repetition rate of the bursts was 120 to 360 per second, and the electron energy was set at a. fixed value in the range of 20 . to $50 \mathrm{MeV}$. For neutron production at 90 degrees to the photon beam the undeflected linac beam was used, while for 45 degree production a bending magnet was used to bring the electrons to a second position for the bremsstrahlung targets.

The bremsstrahlung was produced by stopping the electrons in the beam stopper shown in fig. III-2. The beam stopper consisted of two $0.007^{\prime \prime}$ tantalum sheets separated by $1 / 8^{\prime \prime}$ of water and backed up by $8^{\prime \prime}$ of water to stop al1 of the electrons. For cooling, water was pumped through the beam stopper and a heat exchanger at about $20 \mathrm{gal} / \mathrm{min}$. The bremsstrahlung was 


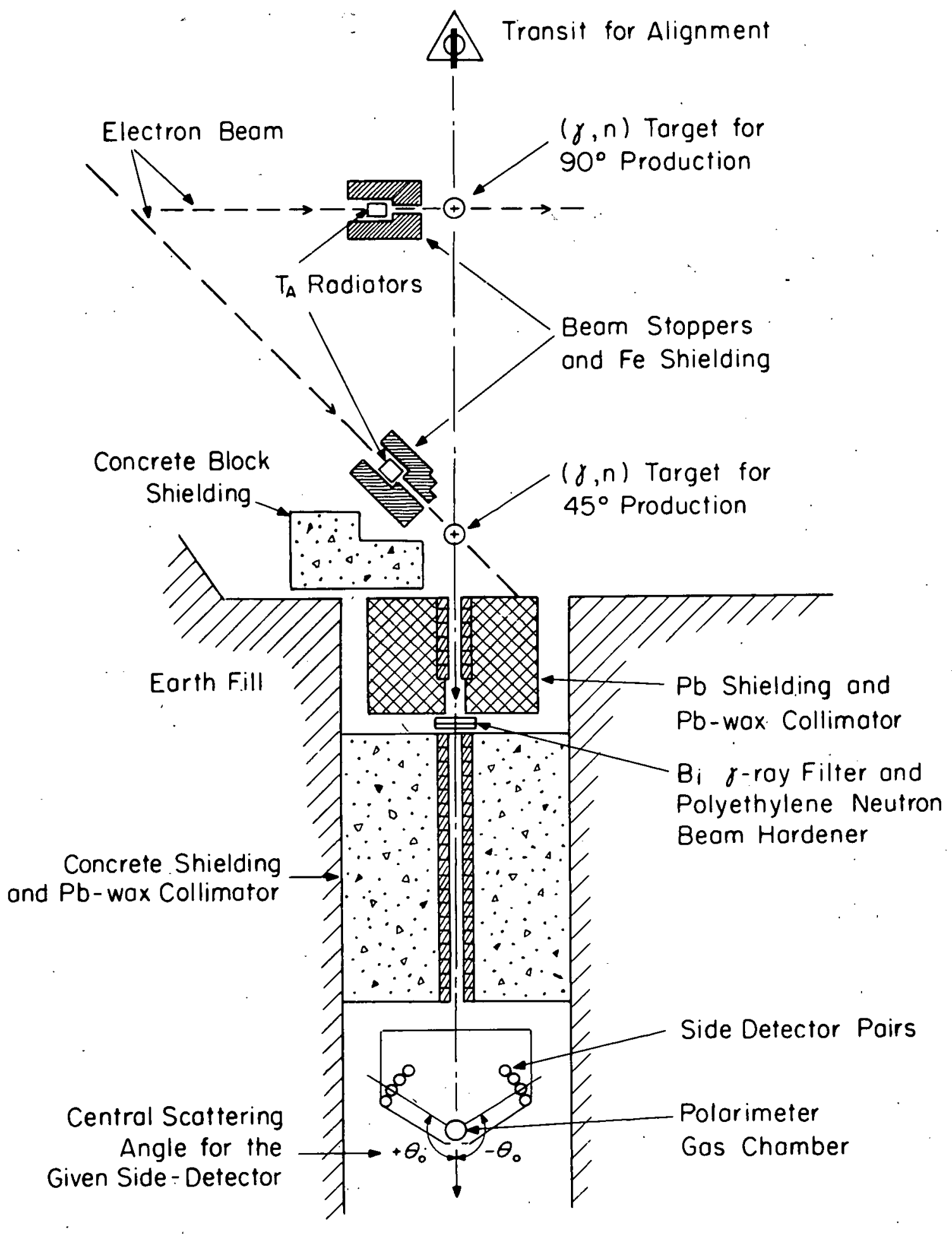

Physical Layout of the Polarization Experiments

Fig. III-I 


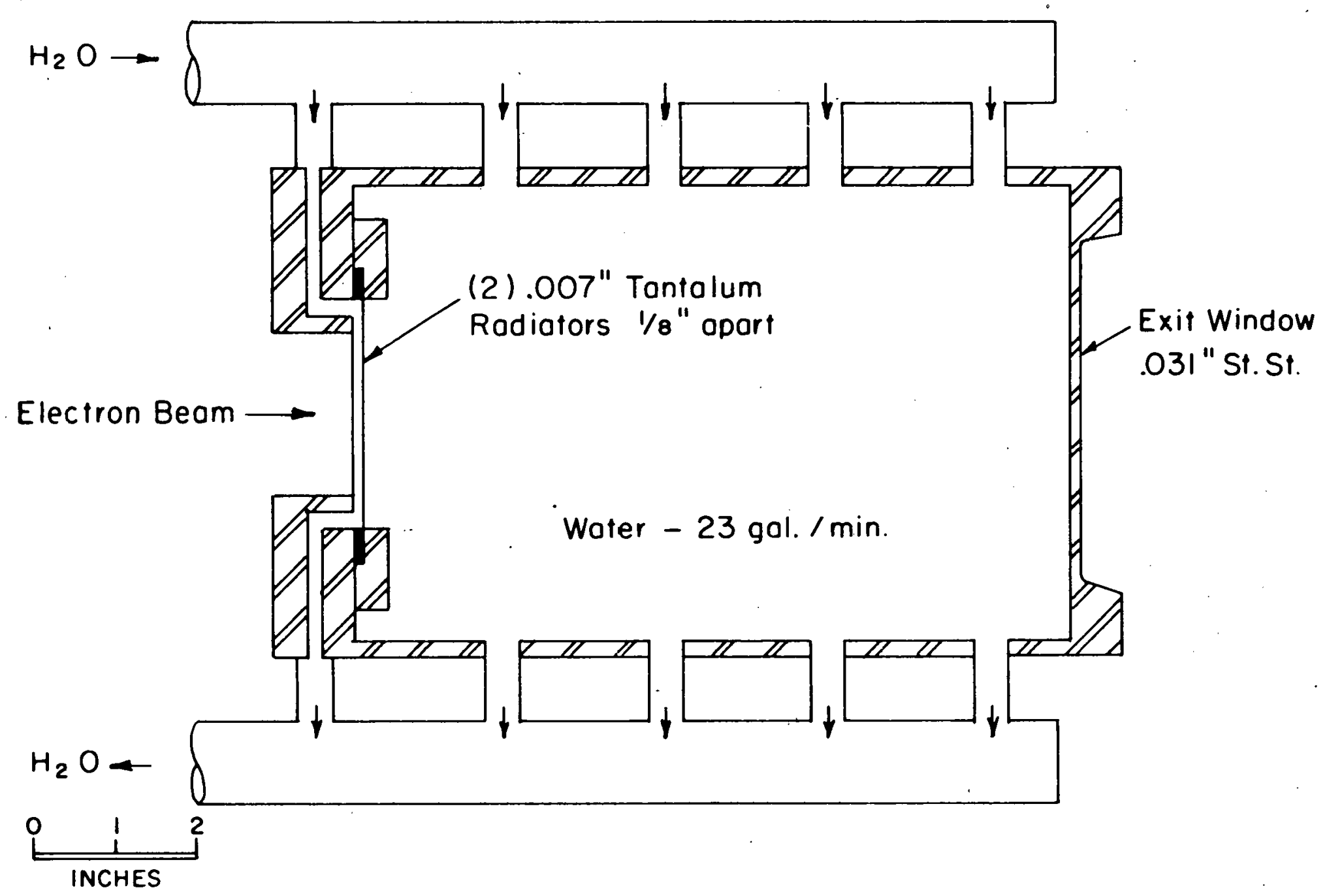

Bremsstrahlung Source and Electron Beam Stopper Fig. III -2 
collimated in the forward direction with about $11 / 2 \mathrm{ft}$ of iron which was designed to produce a beam about $3^{\prime \prime}$ in diameter at the photoneutron target, about 40" away. The remainder of the beam stopper was shielded with $1 / 2$ to $1 \mathrm{ft}$ of iron to reduce neutron production in the target room walls and to shield the polarimeter from the bremsstrahlung.

The photoneutrons were produced in targets suspended in the collimated forward bremsstrahiung beam. The photoneutron targets and several feet of lead and wax collimators defined a neutron beam about $6 "$ in diameter at the polarimeter. The neutron beams were usually filtered by $3^{\prime \prime}$ to $7^{\prime \prime}$ of lead and 1 " or 2 " of polyethylene. The lead was used to reduce the polarimeter background from Compton scattered photons in the neutron beam, while the polyethylene was used as a neutron beam hardener, reducing the number of low energy neutrons. (about $1 \mathrm{MeV}$ ) relative to the number of higher energy neutrons via the energy dependence of the hydrogen cross section. Neutron beam hardening was necessary in some cases to reduce the random counting rate of the polarimeter which was caused by the large number of low energy $\mathrm{He}^{4}$ recoils. This enabled the polarization of the higher energy neutrons to be measured in a reasonable time interval without swamping the polarimeter with low energy pulses.

The polarimeter, which has been described in chapter II, was mounted in the tunnel with the neutron beam irradiating the central portion of the gas-counter, and with the plane of the side-detectors the same as the plane defined by the collimated photon and neutron beams. In addition to the $8^{\prime}$ 
concrete wall, the polarimeter was shielded by a few feet of lead on the target room side of the concrete wall. This helped shield the gas-counter from the bremsstrahlung target, and the side-detectors from the bremsstrahlung and photoneutron targets. A few feet of concrete blocks near the bremsstrahlung target also helped shield the polarimeter. The shielding reduced the $r$-ray counting rate in the sidedetectors to about 1 in 75 machine bursts, and runs made with no photoneutron targets in place gave no measurements of real neutrons. The background levels were thus sufficiently low to make meaningful polarization measurements.

\section{B. Polarimeter Alignment.}

This section will describe the details of polarimeter alignment, and the accuracy of this alignment. In Chapter IV it will be shown that these alignment uncertainties may be neglected when compared with other uncertainties in the polarization measurements.

To make meaningful measurements the polarimeter must be properly aligned w.r.t. the neutron beam. First, the gas-counter must be centered in the neutron beam since otherwise the effective He scattering volume will be located asymmetrically w.r.t. the side-detectors, and thus introduce spurious asymmetry measurements. Second, the side-detectors must be situated at equal scattering angles and distances from the gas-counter since if the two side-detectors of a pair are located inadvertently at different neutron scattering angles a spurious asymmetry can be introduced by the angular dependence in the $\mathrm{He}^{4}(n, n)$ cross section. If the side- 
detectors are at, different distances from the gas-counter an asymmetry will then be introduced by differences in the effective solid angle subtended by each side-detector. Rotation of the side-detector and gas-counter plane about an axis defined by the neutron beam, $\vec{k}_{n}$, introduces an effect smaller than those just mentioned, and tends to reduce the magnitude of measured asymmetries.

To carry out most of the polarimeter alignment, a surveyor's transit was located to sight down the axis of the neutron collimator at the level of the electron beam. The transit was first used to center the gas-counter in the neutron beam. The vertical adjustment was made by raising or lowering the gas-counter until a pencil line on the chamber cylinder'midway between the flanges coincided with the transit cross hair. Horizontal positioning was done by adjusting the cylinder axis to be in line with cross hairs in the collimator.

The 1/2" aluminum plate for mounting the side-detectors was adjusted in height by making it fit properly onto the gas chamber flanges. Previously the flange position had been set by making the gas chamber cylinder axis vertical, which was done by checking with a level against the sides of the cylinder and on the flat portion of the flanges. The error in the right-left position of the gas-countèr w.r.t. the neutron beam is estimated to have been. less than $1 / 8$ "', and about the same for the maximum deviation of the side-detectors from their proper height.

The final adjustment was rotation of the side-detectors 
about the cylinder axis of the gas chamber to set the right and left side-detectors of a pair at equal neutron scattering angles. The alignment in angle was made by using the optical scheme shown in fig. III-3. One. side of a carpenter's level was checked for adequate flatness and then had an optically flat mirror mounted parallel to this side. Machined brass plugs were then placed in two accurately located holes in the $1 / 2 "$ aluminum plate, and the flat side of the level was then placed against the brass plugs with the mirror surface facing the target room. The holes in the aluminum plate were located so that the mirror surface should have been perpendicular to the neutron beam if the plate were aligned to give equal scattering angles. While sighting down the neutron collimator with the transit the aluminum plate was rotated until the reflected image of the transit was centered on its cross hairs. The maximum deviation of the reflected image was about $1 / 4^{\prime \prime}$, in a total light path of about 60', which gives an uncertainty in rotational alignment of about 1 minute of arc. In chapter IV it will be shown that all alignment uncertainties can be neglected in comparison with other sources of uncertainty in the final polarization measurements.

C. Taking Data - List of Targets Used.

To measure the polarization of photoneutrons from a specified element it is necessary to make several polarization measurements. Needed data are:

1) Runs using a target containing the desired element.

2) Runs to measure background from other elements contained in the target and from the target holder. 


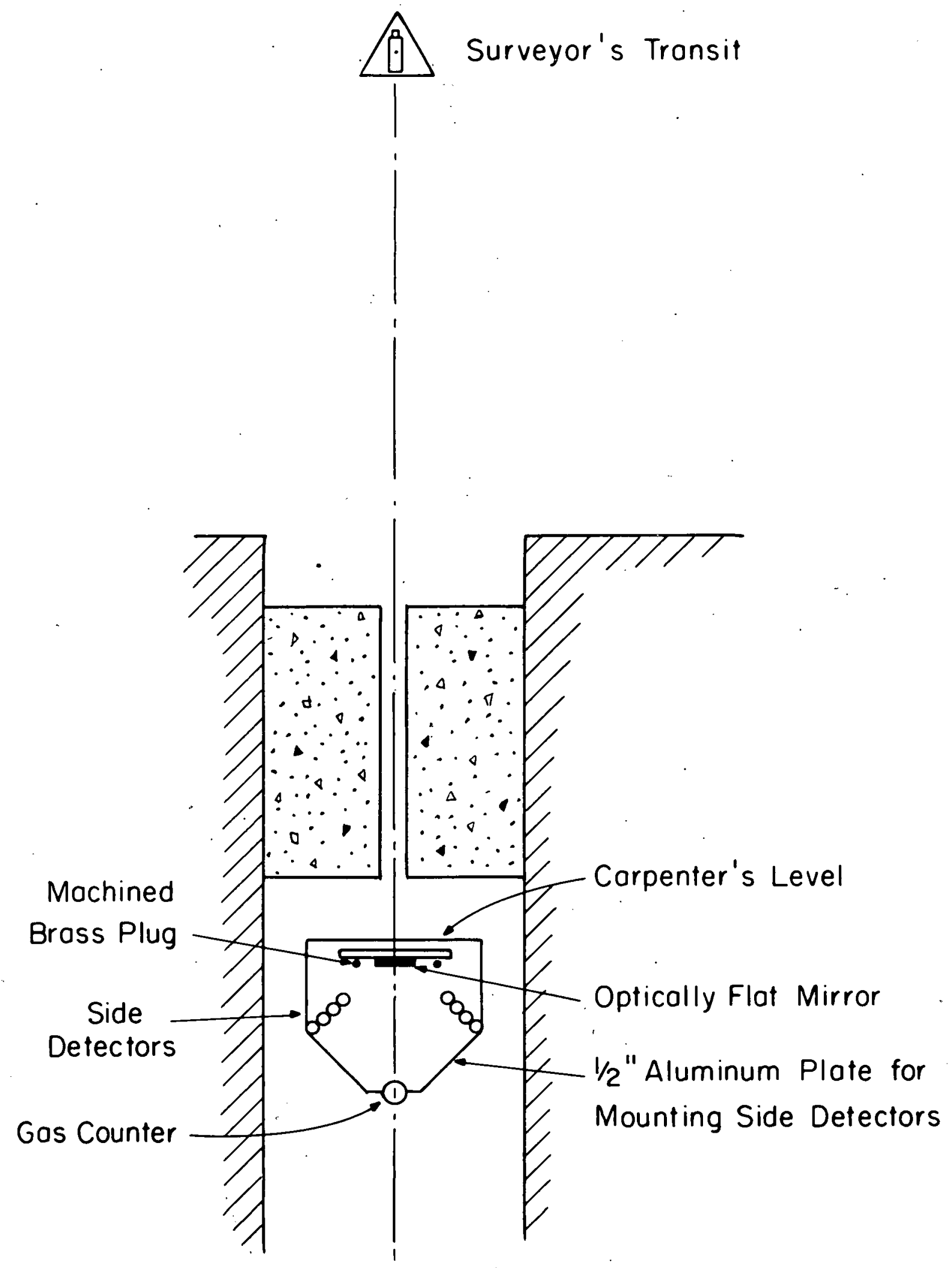

Optical Method for Aligning the Polarimeter

Fig. III -3 
3) Runs to measure background from neutrons produced in the target room walls.

4) Runs to measure background from cosmic rays and natural radioactivity.

5) Estimation of the effects from neutrons produced in the target room and then scattered by the photoneutron target.

To measure the relative yield per photon for proper normalization of background runs, a nitrogen filled ion chamber was mounted in the bremsstrahlung beam a few feet beyond the photoneutron target, and its output used as a measure of the relative photon fluxes.

The first type of runs may be of two forms. Usually a set of $A$ and $B$ runs (a run pair)is used, but as mentioned in Chapter II a single A or B run may also be used to measure polarization.

A second type of run was also of two forms, $A$ and $B$ run pairs, or single runs. They were necessary to subtract the contamination from neutrons produced by other elements in the target used for the first type of runs, and from neutrons produced by the target holder.

The third type of run was made to measure the background from neutrons coming down the collimator after being produced or scattered from the far target room wall which was visible through, the collimator, and from neutrons filtering through the shielding. Measurements showed this to be a negligible source of background.

The fourth type of run was made for several hours with the linac turned off and no coincidence requirements 
with the electron bursts. The results indicate a negligible background for the duty ratios imposed by the coincidence requirements with the accelerator.

The fifth type of background is difficult, usually impossible, to measure, and arises mainly from neutrons produced in the bremsstrahlung collimator being scattered by the photoneutron target. Calculations indicate that this is a negligible source of background.

Two types of runs were made to check for possible biases in the polarimeter electronics. For one case the logic circuits for right and left side-detectors were interchanged by switching cable inputs to the side-detector pulse shapers. In the second case the real-plus-random and random coincidence cijrcuits were interchanged for each side-detector. The first case tested for possible electronic misaddressing of the ADC, which would give rise to spurious polarizations of the opposite sign from the normal electronics arrangement. The second case tested for equality of the real-plus-random and the random coincidence circuit resolving times. These resolving times were also measured by cable insertions to be equal to within $1 / 4 \mathrm{~ns}$. The tests revealed no electronic biases which could produce a spurious polarization greater than about 0.005 .

A 1 ist of all the photoneutron data used in this thesis is given in table III-1. The table lists the desired element, the laboratory photoneutron production angle, the bremsstrahlung end-point energy, the actual target used (type 1 runs) and its shape; and the background target used (type 2 runs) and its 
shape. Where two or more actual targets were used for a single element they are all listed with their corresponding background targets. 


\begin{tabular}{|c|c|c|c|c|c|c|}
\hline $\begin{array}{l}\text { Desired } \\
\text { Element }\end{array}$ & $\begin{array}{l}\text { Lab neutron } \\
\text { production } \\
\text { angle, degrees }\end{array}$ & $\begin{array}{l}\text { Brems.end- } \\
\text { point } \\
\text { energy, } 1 \mathrm{eV} .\end{array}$ & $\begin{array}{l}\text { Actúa } 1 \\
\text { target } \\
\text { used }\end{array}$ & $\begin{array}{l}\text { Shape } \\
\text { of } \\
\text { tar- } \\
\text { get* }\end{array}$ & $\begin{array}{l}\text { Background } \\
\text { target } \\
\text { used }\end{array}$ & $\begin{array}{l}\text { Shape } \\
\text { of } \\
\text { target* }\end{array}$ \\
\hline $0^{16}$ & $\begin{array}{r}45 \\
\\
\\
\\
\\
90\end{array}$ & $\begin{array}{l}24 \\
30 \\
38 \\
30\end{array}$ & $\begin{array}{l}\text { Oxalic acid } \\
\text { Water } \\
\text { Oxalic acid } \\
\text { Water } \\
\text { Liquid oxygen } \\
\text { Liquid oxygen } \\
\text { Water } \\
\text { Oxalic acid } \\
\text { Water }\end{array}$ & $\begin{array}{l}\text { A } \\
\text { A } \\
\text { B } \\
\text { A } \\
\text { C } \\
\text { D } \\
\text { A } \\
\\
\text { B } \\
\text { A }\end{array}$ & $\begin{array}{l}\text { Polyethylene } \\
\text { Polyethylene } \\
\text { Carbon } \\
\text { Polyethylene } \\
\text { Mylar holder } \\
\text { Styrofoam } \\
\text { Polyethylene } \\
\text { none-taken } \\
\text { Polyehtylene }\end{array}$ & $\begin{array}{l}\text { G } \\
\text { G } \\
\text { B } \\
A^{\prime} \cdot \\
C^{\prime} \\
D^{\prime} \\
A^{\prime}(30 \\
\text { Mev run) } \\
\text { alculated } \\
A^{\prime}\end{array}$ \\
\hline $\mathrm{C}^{12}$ & $\begin{array}{l}45 \\
90\end{array}$ & $\begin{array}{l}30 \\
47 \\
50\end{array}$ & $\begin{array}{l}\text { Carbon } \\
\text { Carbon } \\
\text { Carbon }\end{array}$ & $\begin{array}{l}\text { B } \\
\text { B } \\
\text { B }\end{array}$ & $\begin{array}{l}-- \\
-- \\
--\end{array}$ & -- \\
\hline $\mathrm{Ca}^{40}$ & 90 & 28 & Calcium & $E$ & -- & - \\
\hline $\mathrm{Pb}^{208}$ & $\begin{array}{l}45 \\
90\end{array}$ & $\begin{array}{l}28 \\
21\end{array}$ & $\begin{array}{l}\mathrm{Pb}^{208 * *} \\
\mathrm{~Pb}^{208 * *}\end{array}$ & $\begin{array}{l}F \\
F\end{array}$ & -- & $\cdots$ \\
\hline
\end{tabular}

* Symbols signify A - target in a polyethylene bottle, about 2" in dịater and 4 " high.

$A^{\prime}$ - the empty polyethylene bottle used for $A$.

B - a spherical shell of material, about $2 "$ ID and 2 1/2" OD.

C - a cylindrical volume, about $2^{\prime \prime}$ in diameter and 6 " high, made from a mylar cylinder in a vacuum box.

$C^{\prime}$ - the empty mylar target holder for $C$.

D - a cylindrical volume, about 3 " in diameter and 6" high, made by boring a hole in a block of styrofoam.

$D^{\prime}$ - the empty styrofoam 'target holder for $D$.

$E$ - three rods, about 3/4" in diameter and 2" long, mounted next to each other.

F - a circular disc, about 2" in diameter and 1/2" thick.

G - a cylinder 4 " high and $2 "$ in diameter

About $958 \mathrm{~Pb}^{208}$ target obtained on loan from Oak Ridge National Laboratory.

TABLE I I I - I

List of Photoneutron Targets Used for Polarization Measurements 


\section{CHAPTER IV}

\section{METHOD OF DATA ANALYSIS}

Introduction:

The results presented in this thesis come from a total of 52 individual runs. The raw data for one run with four side-detector pairs consist of $16 \mathrm{He}^{4}$ recoil spectra, 8 realplus-random spectra and 8 random spectra. In this chapter we will deal with the treatment of these data to yield neutron polarizations.

In Section A there is a description of how the uncorrected asymmetries and their statistical uncertainties were calculated. In Section $B$ there is a description of how these uncorrected asymmetries were used to calculate the uncorrected polarizations. In Sections $C$ and $E$ these uncorrected poiarizations are treated for various scattering effects, which latter arise from the scattering of neutrons by the polarimeter and tunnel and from neutron scattering in the photoneutron target itself.

In Section D we deal with the problem of background subtraction. Here we describe two methods, which differ only in the order of subtracting background and making corrections for polarimeter and tunnel scattering.

Finally, in Section $F$ the other sources of uncertainty in the polarization are treated. These other uncertainties arise primarily from a possible polarimeter misalignment, and it is shown that they contribute negligibly to the total uncertainty. The results in section $E$ are thus final results of the polarization data analysis. 
The principal sources of uncertainty in the final polarizations are the statistical uncertainties of the uncorracted asymmetries and uncertainties arising from imprècise knowledge of the corrections for polarimeter and tunnel scattering.

A. Calculation of Uncorrected Asymmetries and Their Statistical Uncertainties.

The first stage in the analysis of polarization data is the calculation of uncorrected asymmetries. For neutrons in a specified energy interval, and for a specified side-detector pair, the raw data for a run pair consist of four numbers:

$\mathrm{R}_{\mathrm{RA}}=$ real-plus-random $\mathrm{He}^{4}$ recoils, right side-detector, A run

$A_{R A}=$ randon $\mathrm{He}^{4}$ recoils, right side-detector, $A$ run

$R_{L A}=$ real-plus-randon $\mathrm{He}^{4}$ recoils, left side-detector, A run

$A_{L A}=$ randon $\mathrm{He}^{4}$ recoils, left side-detector, $\mathrm{A}$ run.

For the $B$ run the numbers $R_{R B}, A_{R B}, R_{L B}$ and $A_{L B}$ are obtained. The true numbers of $\mathrm{He}^{4}$ recoils are then

$$
\begin{aligned}
\mathrm{N}_{\mathrm{RA}} & =\mathrm{R}_{\mathrm{RA}}-\mathrm{A}_{\mathrm{RA}} \\
\mathrm{N}_{\mathrm{LA}} & =\mathrm{R}_{\mathrm{LA}}-\mathrm{A}_{\mathrm{LA}} \\
\mathrm{N}_{\mathrm{RB}} & =\mathrm{R}_{\mathrm{RB}}-\mathrm{A}_{\mathrm{RB}} \\
\mathrm{N}_{\mathrm{LB}} & =\mathrm{R}_{\mathrm{LB}}-\mathrm{A}_{\mathrm{LB}} \\
\text { so from eqs } & \text { II.15 and II.14 } \\
\varepsilon & =\frac{\sqrt{\mathrm{N}_{\mathrm{RA} \times} \times{ }_{\mathrm{RB}}}}{\mathrm{N}_{\mathrm{LA}} \mathrm{N}_{\mathrm{LB}}}
\end{aligned}
$$

and

$$
A_{n}=\frac{1-\varepsilon}{1+\varepsilon}=\overline{P_{n} \bar{P}_{H e}}
$$


$\overline{\mathrm{P}_{n} \mathrm{P}_{\mathrm{He}}}$ denotes an appropriate average over the neutrons in the specified energy interval and over the range in scattering angles from the He because of the non-zero He and side-detector volumes. $A_{n}$ is the asymmetry for the specified side-detector pair for neutrons in the selected energy interval.

Since $R_{R A}$, $A_{R A}$, etc. are numbers of $\mathrm{He}^{4}$ recoils with normal counting statistics, the value of $A_{n}$ will have an uncertainty, $\Delta A_{n}$. Using $\sqrt{R_{R A}+1}$ for $\Delta R_{A}$, etc. a straightforward error analysis yields

$$
\begin{aligned}
& \Delta A_{n}=\frac{\varepsilon}{(1+\varepsilon)^{2}}\left[\frac{\left[R_{R A}+A_{R A}+2\right.}{\left(R_{R A}-A_{R A}\right)^{2}}+\frac{R_{R B}+A_{R B}+2}{\left(R_{R B}-A_{R B}\right)^{2}}+\frac{R_{L A}+A_{L A}+2}{\left(R_{L A}-A_{L A}\right)^{2}}\right. \\
& \left.+\frac{R_{L B}+A_{L B}+2}{\left(R_{L B}-A_{L B}\right)^{2}}\right] 1 / 2
\end{aligned}
$$

IV. 2

For a single $A$ or $B$ run the analysis must be modified, and requires the measured detection efficiency ratio from a run pair. If $N_{R A}, N_{L A}, N_{R B}$ and $N_{L B}$ are taken as the run pair, then from eq. II.16 the detection efficiency ratio is

$$
\frac{n_{R A}}{n_{L A}}=\frac{n_{L B}}{n_{R B}}=\sqrt{n_{R A}} \times \frac{\bar{N}_{L B}}{\bar{N}_{L A}}
$$

From the single $A$ run we have data $N_{R A}=R_{R A}-A_{R A}^{\prime}$ and ${ }^{N}{ }_{L A}=R_{L A}-A_{L A}$, and from eq. II.l1 we have

$$
A_{n}^{\prime}=\frac{N_{R A}^{\prime}-N_{L A}^{\prime}\left(\frac{{ }_{R A}}{n_{L A}}\right)}{N_{R A}^{\prime}+N_{L A}\left(\frac{n_{R A}}{n_{L A}}\right)}
$$


The uncertainty in $A_{n}^{\prime}$ is given by

$$
\begin{aligned}
& \Delta A_{n}^{\prime}=\frac{7}{\left(N_{R A}^{\prime \prime}+N_{L A}\left(\frac{n R A}{n L A}\right)\right.}\left[\left(2 N_{L A} \cdot \frac{\left(n_{R A}\right)}{\left(n_{L A}\right)}\right)^{2}\left(R_{R A}^{\prime}+A_{R A}^{\prime \prime}+2\right)\right. \\
& +\left(2 N_{R A}^{\prime}\left(\frac{(n R A)}{n_{L A}}\right)^{2}\left(R_{L A}^{\prime}+A_{L A}^{\prime}+2\right)+\left(2 N_{L A}^{\prime} N_{R A}^{\prime}\right)^{2}\left(\Delta \frac{\left(n_{R A}\right)}{\left(n_{L A}\right)}\right)^{2}\right] 1 / 2
\end{aligned}
$$

IV. 5

where

$$
\begin{aligned}
& \Delta \frac{\left(n_{R A}\right)}{\left(n_{L A}\right)}=\frac{1}{2} \frac{\left(n_{R A}\right)}{\left(n_{L A}\right)} \quad\left[\frac{R_{R A}+A_{R A}+2}{N_{R A}{ }^{2}}+\frac{R_{R B}+A_{R B}+2}{N_{R B}{ }^{2}}+\frac{R_{L A}+A_{L A}+2}{N_{L A}{ }^{2}}\right. \\
& \left.+\frac{R_{L B}+A_{L B}+2}{N_{L B}{ }^{2}}\right] 1 / 2
\end{aligned}
$$

IV. 6

The analysis of a single $B$ run proceeds in the same manner.

For actual polarimeter runs the results in eqs. IV.l and IV.2, or eqs. IV.4 and IV.5, are calculated for several intervals in incident neutron energy, and for each of the four sidedetector pairs. The set of neutron energy intervals is selected according to limitations set by the polarimeter resolution, the level of structure in neutron energy desired, and the minimum number of $\mathrm{He}^{4}$ recoils required to be in an energy interval to give reasonable values for $\Delta A_{n}$.

Since the $\mathrm{He}^{4}$ recoil spectra exist as pulse height spectra in a multichannel analyzer, the incident neutron energy interval set must be converted into analyzer channel sets. These channel sets depend on the central scattering angle of the side-detector pairs and on the corrected calibration channels $\underset{\alpha}{C_{\alpha}^{\prime}}$ and $\mathrm{C}_{\alpha}^{\prime} / 2$ of eq. II.17.

The channel corresponding to a neutron of energy $E_{n} \mathrm{MeV}$ is 


$$
C_{n}=\left[2 C_{\alpha / 2}^{\prime}-C_{\alpha}^{\prime}\right]+\frac{\left(C_{\alpha}^{\prime}-C_{\alpha / 2}^{\prime}\right)}{2.65}\left[E_{n}\left[1-\frac{\left(\sqrt{\cos ^{2} \cdot \theta_{0}+14.76}+\cos \theta_{0}\right)^{2}}{24.7}\right]\right]
$$

IV .7

where the results of ea. II.8 have been used with $\rho=\frac{m_{n}}{m_{H_{\mathrm{H}}}}=0.252$, and $\theta_{0}$ is the central laboratory scattering angle for the sidedetector pair in question. With eq. IV.7 the incident neutron energy interval set can be converted into channel-sums of the analyzer spectra, and the asymmetries calculated.

The final results of this stage of data analysis are four sets of asymmetries and uncertainties, one set for each side.. detector pair. The four sets will be called $A_{n i}\left(\theta_{j}\right) \pm \Delta A_{n i}$ $\left(\theta_{j}\right)$, where $i$ stands for the $i$ 'th neutron energy interval and $\theta_{j}$ is the central scattering angle of the $j^{\prime}$ th side-detector pair.

\section{B. Calculation of the Uncorrected Neutron Polarization} and Spectrum

The asymmetries are a suitably averaged product of the neutron polarization and the $\mathrm{He}^{4}(\mathrm{n}, \mathrm{n})$ polarizing power. From eq. IV.l we have for the side-detector pair with central scattering angle $\theta_{0}$

$$
A_{n}=\overline{\mathrm{P}_{n} \mathrm{P}_{\mathrm{He}}\left(\theta_{0}\right)}
$$

IV .8

If $\frac{d N}{d E_{n}}$ is the differential neutron spectrum incident on the $\mathrm{He}^{4}$ polarimeter, then

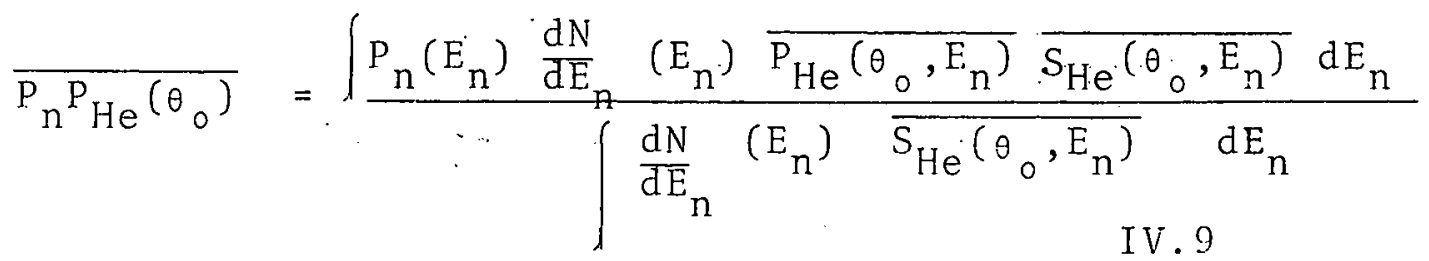


where $\overline{\mathrm{P}_{\mathrm{He}}\left(\theta_{0}, \mathrm{E}_{\mathrm{n}}\right)}$ and $\overline{\mathrm{S}_{\mathrm{He}}\left(\theta_{0}, \mathrm{E}_{\mathrm{n}}\right)}$ are the polarizing power and detection efficiency of the polarimeter, for incident neutrons of energy $E_{n}$ and the side-detector pair at scattering angle $\theta_{0}$. $\overline{\mathrm{P}_{\mathrm{He}}\left(\theta_{0}, E_{n}\right)}$ and $\overline{\mathrm{S}_{\mathrm{He}}\left(\theta_{0}, E_{n}\right)}$ are averaged over the scattering volume of the He and the detection volume of the side-detector. The details of this calculation are given in Appendix II. The integrations in eq. IV.9 go over the neutron energy interval in question.

If the neutron energy interval is such that the variations in $P_{n}, \frac{d N}{d E_{n}}, \overline{P_{H e}}$ and $\bar{S}_{H e}$ are sufficiently small, then eq.IV.9 can be rewritten as

$$
A_{n}\left(\overline{E_{n}}\right) \simeq \overline{P_{n}}\left(\overline{E_{n}}\right) \overline{P_{H e}}\left(\theta_{0}, \overline{E_{n}}\right) \quad \text { IV. } 10
$$

where $\overline{E_{n}}$ is the average energy of the neutrons in the given interval and

$$
\begin{aligned}
& \overline{P_{n}}\left(\overline{E_{n}}\right)=\int \frac{\int P_{n}\left(E_{n}\right) \frac{d N}{d E_{n}}\left(E_{n}\right) d E_{n}}{\int \frac{d N}{d E_{n}\left(E_{n}\right) d E_{n}}} \quad I V .11
\end{aligned}
$$

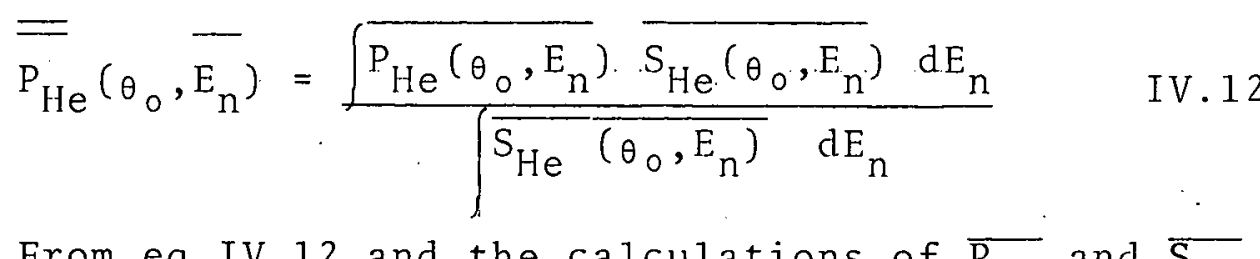

From eq.IV.12 and the calculations of $\overline{\mathrm{P}_{\mathrm{He}}}$ and $\overline{\mathrm{S}_{\mathrm{He}}}$ discussed in appendix II the values of $\overline{\overline{\mathrm{P}_{\mathrm{He}}}}$ can be calculated, and with eq.IV.10 give

$$
\overline{P_{n}}\left(\overline{E_{n}}\right)=\frac{A_{n}\left(\overline{E_{n}}\right)}{\overline{\overline{P_{H e}}}\left(\theta_{0}, \bar{E}_{n}\right)}
$$

The values of $\overline{\overline{\mathrm{P}_{\mathrm{He}}}}\left(\theta_{0}, \overline{\mathrm{E}_{\mathrm{n}}}\right)$ were calculated from the phase shifts of Hoop and Barschall (53), so eq.IV.13 gives $\bar{P}_{n}$ from a measured quantity and a calculated quantity. 
The relative neutron spectrum incident on the polarimeter can also be calculated.

If we set

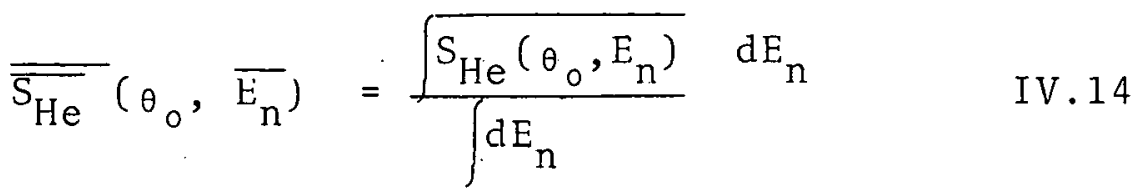

as the average detection efficiency of the polarimeter, for the given side-detector pair and neutron energy interval, then using the number of $\mathrm{He}^{4}$ recoils for a run pair as defined in Section A we have

$$
\bar{N}_{n}\left(\bar{E}_{n}\right)=\frac{N_{R A}+N_{L A}+N_{R B}+N_{L B}}{\overline{\bar{S}_{\mathrm{He}}}\left(\theta_{0}, \overline{\mathrm{E}}_{n}\right)} \quad \text { IV.15 }
$$

where $\overline{\mathrm{N}}_{\mathrm{n}}\left(\overline{\mathrm{E}}_{\mathrm{n}}\right)$ is the relative number of neutrons in the neutron. energy interval centered on $\bar{E}_{n}$.

In the absence of any corrections to the data, $\overline{\mathrm{P}}_{n}$ and $\overline{\mathrm{N}}_{\mathrm{n}}$ would be independent of the side-detector pair used to make. the measurement. However, polarimeter and tunnel scattering corrections make $\overline{\mathrm{P}}_{\mathrm{n}}$ and $\overline{\mathrm{N}}_{\mathrm{n}}$ also dependent on the side-detector scattering angle, so the uncorrected neutron polarization and spectrum for a run pair actually consist of four sets, one from each side-detector pair. These four sets, which will be labeled $\overline{P_{n}}\left(\theta_{j},{\overline{E_{n}}}_{i}\right)$ and $\bar{N}_{n}\left(\theta_{j}, \bar{E}_{n_{i}}\right)$, form the input for the analysis stage described in section $C$.

One final detail for this section is the calculation of uncertainties for $\overline{\mathrm{P}}_{\mathrm{n}}$ and $\overline{\mathrm{N}}_{\mathrm{n}}$. An error analysis (assuming $\Delta \overline{\mathrm{P}}_{\text {Ile }}=0$ ) yields

$$
\Delta \bar{P}_{n}=\frac{\Delta A_{n}\left(\bar{E}_{n}\right)}{\left.\overline{\overline{P_{H e}}}{ }^{i \theta_{0}}, E_{n}\right)}
$$

and

$$
\Delta \bar{N}_{n}=\frac{\left\{\left(R_{R A}+A_{R A}+2\right)+\left(R_{E A}+A_{L A}+2\right)+\left(P_{R B}+A_{R B}+2\right)+\left(R_{L B}+A_{L B}+2\right)\right\}}{\bar{S}_{H e}\left(\theta_{0}, \bar{E}_{n}\right)}
$$


In this thesis no error has been included for $\overline{\overline{\mathrm{Pe}}}$ and $\overline{\overline{\mathrm{He}}}$. In view of the analysis by $\mathrm{S}$. Kowalski (46) and the new $\mathrm{He}^{4}(\mathrm{n}, \mathrm{n})$ phase shifts of Hoop and Barschall(53), the uncertainties in $\overline{\overline{\mathrm{Pe}}}$ and $\overline{\overline{\mathrm{He}}}$ have been assumed small compared to those introduced by the polarimeter and tunnel scattering corrections.

Táble IV-1. shows the values of $\left(\frac{\Delta P}{P}\right)$ He which were used by S. Fowalski. For measurements made with the stainless steel gas chamber with side-detectors at $113^{\circ}, 127^{\circ}, 141^{\circ}$ and $155^{\circ}$, and about the same for the aluminum gas chamber which generally utilized the angles given in Table IV-1, the values from this table indicate that $\left(\frac{\Delta \mathrm{P}}{\mathrm{P}}\right)_{\mathrm{He}}$, averaging the data over all the angles, is roughly 0.05 . The largest measured polarization $\mathrm{P}_{\mathrm{n}}$ was about $0.50 \pm 0.05$. The uncertainty $\Delta \mathrm{P}_{\mathrm{n}}$ caused by. $\left(\frac{\Delta \mathrm{P}}{\mathrm{P}}\right)_{\mathrm{He}}=.05$, is \pm 0.025 . This contribution would only give a $12 \%$ increase to the uncertainty of \pm 0.05 for $\Delta \mathrm{P}_{\mathrm{n}}$ which is used above. Most measured polarizations were about 0.10 and have an uncertainty $\Delta \mathrm{P}_{\mathrm{n}}$ from $\left(\frac{\Delta \mathrm{P}}{\mathrm{P}}\right)_{\mathrm{He}}$ of about \pm 0.005 , which is negligible when combined with the $\Delta \mathrm{P}_{\mathrm{n}}$ caused by other sources (e.g. statistics).

C. Corrections Due to Polarimeter and Tunnel Scattering

The corrections due to polarimeter and tunnel scattering arise chiefly from double neutron scatterings. A neutron in the incident beam may scatter first from the He and then from the gas chamber or tunnel wall, with some of the second scatterings resulting in the eventual triggering of a sidedetector. Many of these double scatterings satisfy the timing conditions for a real-plus-random event and thus appear as part of the measured $\mathrm{He}^{4}$ recoil spectra. 


\section{$\mathrm{He}^{4}(\mathrm{n}, \mathrm{n}) \mathrm{He}^{4}$ Polarization Errors}

Estimated error in $\mathrm{P}_{\mathrm{He}}, \pm\left(\frac{\Delta \mathrm{P}}{\mathrm{P}}\right)$ (from S. Kowalski)

\begin{tabular}{|c|c|c|c|c|}
\hline $\begin{array}{c}\text { Neutron Energy / Side-detector } \\
\text { (MeV) }\end{array}$ & $47^{\circ}$ & $67^{\circ}$ & $117^{\circ}$ & $137^{\circ}$ \\
\hline 3.8 & - & .06 & .02 & .10 \\
\hline 5.6 & .06 & .06 & .02 & .10 \\
\hline $7.5^{\prime}$ & .06 & .06 & .02 & .10 \\
\hline 10.7 & .15 & .08 & .05 & .10 \\
\hline
\end{tabular}

TABLE IV-1 
Corrections to the measured polarization data will first be considered in a general form. This general form will be used for the background subtraction and the photoneutron target scattering corrections described in Sections $D$ and $E$, as well as for the polarimeter and tunnel scattering corrections described in the present section.

Corrections to measured neutron polarizations may be defined as follows. Assume a monoenergetic photoneutron beam of $\mathrm{N}_{n}$ neutrons with $\mathrm{E}_{\mathrm{n}}$ MeV polarized along $\underset{\mathrm{k}_{\gamma}}{\overrightarrow{\mathrm{N}}_{\mathrm{r}}} \times \overrightarrow{\mathrm{k}}_{\mathrm{n}}$, and let the numbers with spin projection $\pm 1 / 2$ along $\mathrm{k}_{\gamma} \times \mathrm{k}_{n}$ be $\mathrm{N}_{\mathrm{n} \pm}$. Then the polarization of this beam is -

$$
P_{n}=\frac{N_{n}-N_{n-}}{N_{n+}+N_{n-}-}
$$

Let us further assume that there is a second, contaminating, source of neutrons of apparent energy $\mathrm{E}_{\mathrm{n}} \mathrm{MeV}$, (they produce the same energy $\mathrm{He}^{4}$ recoil) and that this source has a measured polärization along $\vec{k}_{\gamma} \times \vec{k}_{n}$ of

$$
P_{c}=\frac{N_{c^{+}}-N_{c_{-}}}{N_{c^{+}}+N_{c_{-}}}
$$

The polarimeter will be unable to distinguish the two kinds of neutrons, as both have apparent energy $E_{n}$, so the measured polarization along $\vec{k}_{\gamma} \times \vec{k}_{n}$ will be

$$
P_{m}=\frac{\left(N_{n+}+N_{c^{+}}\right)-\left(N_{n-}+N_{c_{-}}\right)}{\left(N_{n+}+N_{c^{+}}\right)+\left(N_{n-}+N_{c_{-}}\right)}
$$


which can be rewritten as

$$
\mathrm{F}_{\mathrm{m}}=\left(1-\mathrm{k}_{\mathrm{c}}\right) \mathrm{P}_{\mathrm{n}}+\mathrm{k}_{\mathrm{c}} \mathrm{P}_{\mathrm{c}} \quad \text { IV. } 21
$$

where

$$
k_{c}=\frac{N_{c+}+{ }^{N}{ }_{c-}}{N_{n+}+N_{n-}+N_{c+}+N_{c-}} \quad \text { IV. } 22
$$

Eq. IV.21 can be solved for $P_{n}$ to give

$$
P_{n}=\frac{P_{m}-k_{c} P_{c}}{1-k_{c}}
$$

so if $\mathrm{P}_{c}$ and $k_{c}$ can be measured or calculated, $P_{n}$ may bé found from $P_{m} \cdot k_{c}$ is the fraction of contaminating neutrons and $P_{C}$ is the polarization of this fraction.

The measured polarization and spectra of Section $B$, $\bar{P}_{n}\left(\theta_{j}, \bar{E}_{n i}\right)$ and $\bar{N}_{n}\left(\theta_{j}, \bar{E}_{n i}\right)$, are corrected for polarimeter and tunnel scattering with eq. IV. 23. For a specified side-detector pair we may consider $\theta_{j}$ as fixed and define.

$$
\begin{aligned}
& P_{m}(i)=P_{n}\left(\theta_{j}, E_{n i}\right) \\
& N_{m}(i)=N_{n}\left(\theta_{j}, E_{n i}\right)
\end{aligned}
$$

The fraction of contaminating neutrons $k_{c}$ and their polarization $P_{c}$ must be calculated from $P_{m}(i)$ and $N_{m}(i)$, where $i$ is the index which labels the neutron energy spectrum.

The fraction $k_{c}\left(\equiv k_{s c}\right)$ and polarization $P_{C}\left(\equiv P_{s c}\right)$ for polarimeter and tunnel scattering corrections are calculated from four matrices $M_{S C^{+}}(i, j), M_{s C^{-}}(i, j), P_{s c^{+}}(i, j)$ and $P_{s c-}(i, j)$. The details of calculating these matrices are given in chapter VI. $M_{s c_{+}}(i, j), M_{s c_{-}}(i, j), P_{s c^{+}}(i, j)$ and 
$P_{S C-}(i, j)$ relate the measured polarization and spectrum, $\mathrm{P}_{\mathrm{m}}(\mathrm{i})$ and $\mathrm{N}_{\mathrm{m}}$ (i), to the polarization and spectrum of the neutron beam incident on the polarimeter, $\mathrm{P}_{\text {in }}(\mathrm{i})$ and $\mathrm{N}_{\text {in }}(\mathrm{i})$, by the equations

$$
\begin{aligned}
& N_{m}(i)=\frac{N_{i n}(i)}{1-k_{s c}(i)} \\
& P_{m}(i)=P_{s c}(i) k_{s c}(i)+P_{i n}(i)\left(1-k_{s c}(i)\right)
\end{aligned}
$$

where

$$
\begin{aligned}
& N_{S C^{ \pm}}(i)=\sum_{j} N_{i n}(j) M_{S C^{ \pm}}(i, j) \cdot\left[\frac{1 \pm P_{i n}(j)}{2}\right] \\
& P_{S C \pm}(i)=\sum N_{j}(j) M_{S C \pm}(i, j,) P_{S C \pm}(i, j)\left[\frac{1 \pm P_{\text {in }}(j)}{2}\right] / N_{S C \pm}(i) \\
& P_{s C}(i)=\frac{N_{s c+}(i) P_{s C+}(i)+N_{s c-}(i) P_{s c-}(i)}{N_{s c+}(i)+N_{s c-}(i)} \\
& k_{s c}(i)=\frac{N_{s C+}(i)+N_{s c-}(i)}{N_{i n}(i)+N_{s c+}(i)+N_{s c-}(i)}
\end{aligned}
$$

Using eqs. IV.25 and IV.26 the polarization and spectrum of the incident neutron beam, corrected for polarimeter and tunnel scattering, are

$$
\begin{aligned}
P_{\text {in }}(i) & =\frac{P_{m}(i)-P_{s c}(i) k_{s c}(i)}{1-k_{s c}(i)} \\
N_{\text {in }}(i) & =N_{m}(i)-N_{s C+}(i)-N_{s C-}(i) \\
& =N_{m}(i)\left(1-k_{s c}(i)\right)
\end{aligned}
$$

IV. 27

In practice eqs. IV.27 cannot be solved directly for $\mathrm{P}_{\text {in }}(\mathrm{i})$ and $N_{\text {in }}(i)$ since, $P_{S C}(i)$ and $k_{s c}(i)$ are functions of $P_{\text {in }}(i)$ 
$\mathrm{N}_{\text {in }}(\mathrm{i}) \cdot$ Thus a reiterative method was used to solve eqs. IV. 27 .

A zero order solution for $\left(\mathrm{P}_{\text {in, }} \mathrm{N}_{\text {in }}\right)$ was taken to be $\left(\mathrm{P}_{\mathrm{m}}, \mathrm{N}_{\mathrm{m}}\right)$, and used to obtain zero order values for $\mathrm{k}_{\mathrm{sc}}$ and $\mathrm{P}_{\mathrm{sc}}$ with eqs. IV.26. These zero order $k_{s c}$ and $P_{s c}$ were then used on the right side of eqs. IV.27, yielding a first order estimate to $\left(\mathrm{P}_{\text {in }}, \mathrm{N}_{\text {in }}\right)$, which may be labeled $\left(\mathrm{P}_{1}, \mathrm{~N}_{1}\right)$. These $\left(\mathrm{P}_{1}, \mathrm{~N}_{1}\right)$ were then used in eqs. IV.26 to obtain first order $\mathrm{k}_{\mathrm{Sc}}$ and $\mathrm{Ps}_{\mathrm{c}}$, which were then used in eq.IV.27 to obtain second order estimates for $\left(\mathrm{P}_{i_{n}}, \mathrm{~N}_{\text {in }}\right)$. The reiteration was repeated twice more to obtain the final estimate to the corrections. The difference in the values of $\left(\mathrm{P}_{3}, \mathrm{~N}_{3}\right)$ and $\left(\mathrm{P}_{4}, \mathrm{~N}_{4}\right)$ were a few percent or less so the final solution of eqs. IV.27 for $\left(\mathrm{P}_{\text {in }}, \mathrm{N}_{\text {in }}\right)$ was consistent to a few percent in the magnitude of $P_{\text {in }}$ and $N_{\text {in }}$.

The final corrections with eqs. IV. 27 were made with $\mathrm{k}_{\mathrm{SC}}$ and $\mathrm{P}_{\mathrm{SC}}$ in eq.IV.26 calculated from the average of $\left(\mathrm{P}_{4}, \mathrm{~N}_{4}\right)$ over. the four side-detector pairs. Since $\left(\mathrm{P}_{\text {in }}, \mathrm{N}_{\text {in }}\right)$ are properties of the neutron beam incident on the polarimeter the four sets of $\left(\mathrm{P}_{4}, \mathrm{~N}_{4}\right)$ are separate estimates of the same quantities and their average should be a better estimate. The agreement among the four sets of $\left(\mathrm{P}_{4}, \mathrm{~N}_{4}\right)$ is shown in fig. IV-1 where they are plotted along with the average, $\left(\overline{\mathrm{P}}_{4}, \overline{\mathrm{N}}_{4}\right)$, for an actual run pair. The average, $\left(\overline{\mathrm{P}}_{4}, \overline{\mathrm{N}}_{4}\right)$, was used in eqs.IV.26 to make the final corrections in eq.IV. 27

Once the polarimeter and tunnel scattering corrections have been made to the polarization measurements it is necessary 


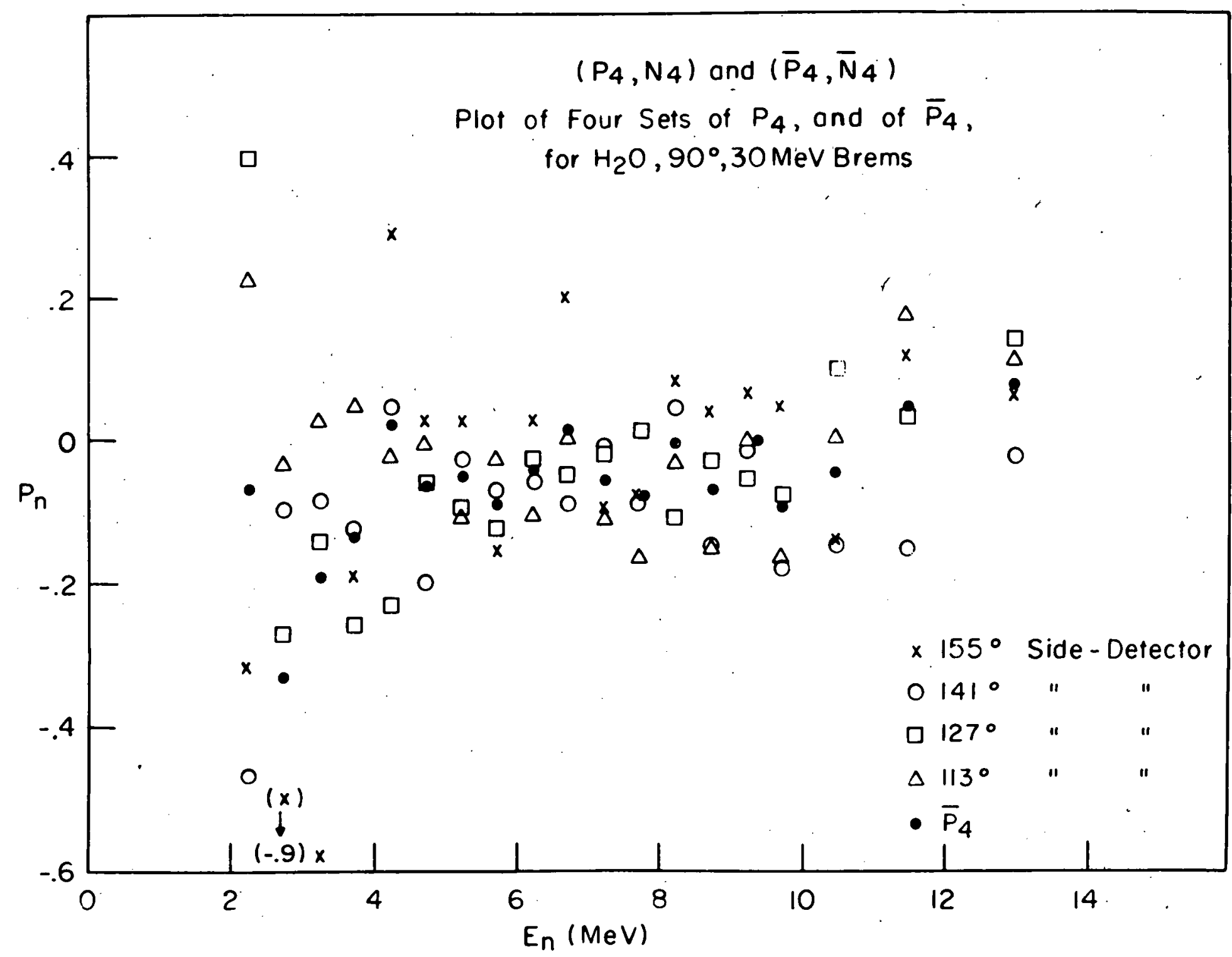

Plots of the Four Sets of $\left(\mathrm{P}_{4}, \mathrm{~N}_{4}\right)$ and of $\left(\overline{\mathrm{P}}_{4}, \overline{\mathrm{N}}_{4}\right)$

Fig. IV-1a 


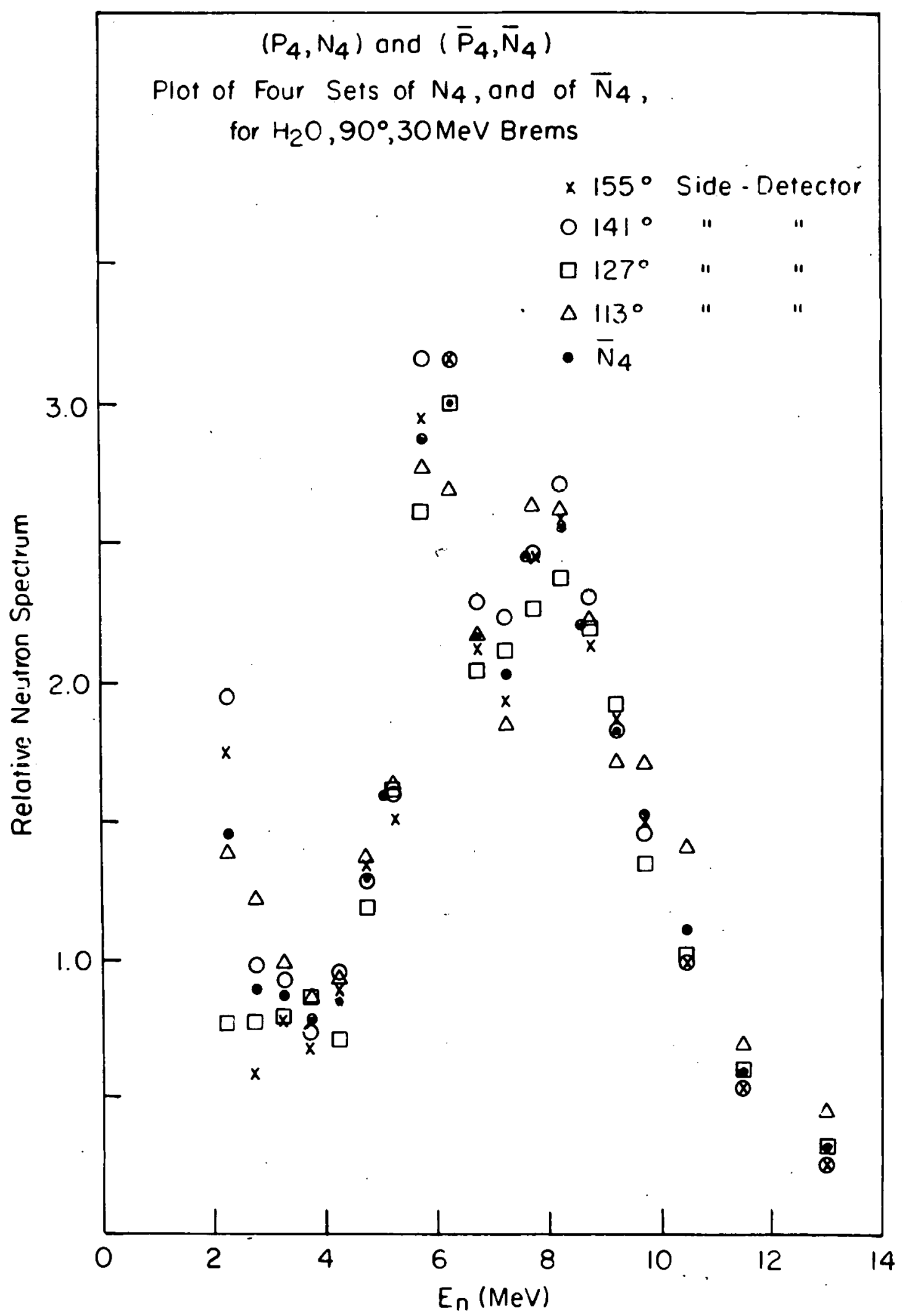

Plots of the Four Sets of $\left(\mathrm{P}_{4}, \mathrm{~N}_{4}\right)$ and of $\left(\overline{\mathrm{P}}_{4}, \overline{\mathrm{N}}_{4}\right)$

Fig. IV-Ib 
to calculate uncertainties for the corrected quantities. Eqs. IV.16 and IV.17 give the statistical êrrors, $\Delta \bar{P}_{n}$ and $\Delta \bar{N}_{n}$, for the four sets of measured $\left(\mathrm{P}_{m}, N_{m}\right)$. If the uncertainties in $\mathrm{P}_{S C}(i)$ and $\cdot k_{S C}(i)$ are $\Delta P_{S C}(i)$ and $\Delta k_{S C}(i)$, then for a specified side-detector pair we have

$$
\begin{aligned}
\Delta P_{\text {in }}(i)_{s t}= & \frac{\Delta \bar{P}_{n}(i)}{1-k_{s c}(i)} \\
\Delta P_{\text {in }}(i)_{s y s}= & {\left[\left[\frac{k_{s c}(i)}{l-k_{s c}(i)}\right)^{2}\left(\Delta P_{s c}(i)\right)^{2}+\left(\frac{P_{m}(i)-P_{s c}(i)}{\left(1-k_{s c}(i)\right)^{2}}\right)^{2}\right.} \\
\times & \left.\left(\Delta k_{s c}(i)\right)^{2}\right] 1 / 2 \\
\Delta P_{\text {in }}(i)= & {\left[\left(\Delta P_{i n}(i) s t\right)^{2}+\left(\Delta P_{i n}(i) s y s\right)^{2}\right] 1 / 2 }
\end{aligned}
$$

IV. 28

and

$$
\begin{aligned}
& \Delta N_{\text {in }}(i)_{s t}=\Delta \bar{N}_{n}(i)\left(1-k_{s C}(i)\right) \\
& \Delta N_{\text {in }}(i)_{\text {sys }}=\Delta N_{m}(i) \Delta k_{s c}(i) \quad \text { IV. } 29 \\
& \Delta N_{\text {in }}(i)=\left[\left(\Delta N_{\text {in }}(i)_{s t}\right)^{2}+\left(\Delta N_{\text {in }}(i)_{\text {sys }}\right)^{2]}\right] 1 / 2
\end{aligned}
$$

where the subscript "st" stands for statistical error component and "sys". for systematic error component.

The uncertainties were split into two components to distinguish which part came from the counting statistics and which part came from the scattering corrections. The systematic 
error in the polarization measurements arises from uncertainties in the scattering corrections. As is shown in chapter VI, the scattering correction matrices are estimated to be uncertain by about $30 \%$, so

$$
\begin{aligned}
& \Delta k_{S C}=0.3 \cdot k_{S C} \\
& \Delta P_{S C}=0.3 P_{S C}
\end{aligned}
$$

IV. 30

Since the scattering correction matrices are caiculated in the same manner for each side-detector pair the uncertainties $\Delta \mathrm{k}_{\mathrm{SC}}$ and $\Delta \mathrm{P}_{\mathrm{SC}}$ have a correlated component for the four sidedetector pairs, and this must be taken into account when averaging the four results to obtain the averaged and corrected polarization measurements for a given run set.

A general method of combining a set of measurements with statistical (or uncorrelated) and systematic (or correlated) components in their uncertainties will now be described. We consider a specific neutron energy interval centered on $\overline{\mathrm{E}}_{\mathrm{n}}$, and label the four side-detector pairs with a subscript $j$. The four final polarization measurement results for $\bar{E}_{n}$ are then $P_{j}, \Delta P_{j}$, $\Delta \mathrm{P}_{\text {stj }}$, and $\Delta \mathrm{P}_{\text {sys }}{ }_{j} \quad$ We weight the polarizations with the inverse square of the statistical uncertainty, and then an error analoysis gives, assuming complete correlation of the systematic error components,

$$
\begin{aligned}
w_{j} & =\frac{1}{\left(\Delta P_{s t_{j}}\right)^{2}} \\
\bar{P} & =\frac{\Sigma_{j}{ }_{j} P_{j}}{\Sigma_{j} w_{j}} \\
\Delta \bar{P}_{s t} & =\frac{1}{\left[\Sigma_{j} w_{j}\right] 1 / 2}
\end{aligned}
$$




$$
\begin{aligned}
\Delta \overline{\mathrm{P}}_{\text {sys }} & =\frac{\sum_{i} w_{j} \Delta \mathrm{P}_{\text {sys }}}{\bar{\Sigma}_{j} \mathrm{w}_{j}} \\
\Delta \overline{\mathrm{P}} & =\left[\left({ } \overline{\mathrm{P}}_{s t}\right)^{2}+\left(\Delta \overline{\mathrm{P}}_{\text {sys }}\right)^{2}\right] 1 / 2
\end{aligned}
$$

The same method is used for $\bar{N}, \Delta \bar{N}_{s t}, \Delta \bar{N}_{s y s}$, and $\Delta \bar{N}$, the corrected and averaged spectrum of the neutrons incident on the polarimeter.

The final results of subtracting the corrections for polarimeter and tunnel scattering are the polarization and spectrum

$$
\begin{aligned}
\overline{\mathrm{P}}\left(\overline{\mathrm{E}}_{\mathrm{n} i}\right) & \pm \Delta \overline{\mathrm{P}}\left(\overline{\mathrm{E}}_{n \dot{\mathrm{i}}}\right) \\
\Delta \overline{\mathrm{P}}\left(\overline{\mathrm{E}}_{\mathrm{n} i}\right) & =\left[\left(\Delta \overline{\mathrm{P}}_{\mathrm{st}}\left(\overline{\mathrm{E}}_{\mathrm{n} \dot{\mathrm{P}}}\right)\right)^{2}+\left(\Delta \overline{\mathrm{P}}_{\mathrm{sys}}\left(\overline{\mathrm{E}}_{n i}\right)\right)^{2] 1 / 2 .}\right.
\end{aligned}
$$

IV. 32

and

$$
\begin{aligned}
& \bar{N}\left(\bar{E}_{n i}\right) \pm \Delta \bar{N}\left(\bar{E}_{n i}\right) \\
& \Delta \bar{N}\left(\overline{E_{n i}}\right)=\left[\left(\Delta \bar{N}_{s t}\left(\overline{E_{n i}}\right)\right)^{2}+\left(\Delta \bar{N}_{s y s}\left(\overline{E_{n i}}\right)\right)^{2}\right] 1 / 2
\end{aligned}
$$

IV. 33

where $i$ goes.over the entire range of neutron energy intervals selected in the first stage of data analysis. These results are the starting point for later stages of analysis; now to be described in Sections $\Omega$ and $E$.

D. Subtraction of Background

If the photoneutron target has some contaminating neutronproducing element in it, or if the target holder produces neutrons, 
then these neutrons form background. Background is best illustrated by the oxalic acid $\left(\mathrm{C}_{2} \mathrm{H}_{2} \mathrm{O}_{2} \cdot 2 \mathrm{H}_{2} \mathrm{O}\right)$ targets which were used for some of the $0^{16}(\gamma, n)$ polarization measurements, where the carbon also produces neutrons. The neutron polarization measurements on oxalic acid must be corrected for the carbon neutrons before results for oxygen are obtained. Background is measured by making runs with the desired target (e.g. oxalic acid), and runs with either a target of the contaminating element (carbon for the oxalic acid) or with the empty target holder. The relative integrated bremsstrahlung fluxes were measured with the ion-chamber monitor described in chapter III.

The method of background subtraction will now be illustrated with the example of subtracting the carbon contamination of the oxygen photoneutrons from an oxalic acid target. Let the results of eqs. IV.32 and IV.33 for a set of oxalic acid runs be $\overline{\mathrm{P}}_{\text {ox }}{ }^{ \pm}$ $\Delta \overline{\mathrm{P}}_{o x}, \bar{N}_{o x} \pm \Delta \bar{N}_{o x}$, and for the background carbon runs $\overline{\mathrm{P}}_{C}{ }_{\Delta} \overline{\mathrm{P}}_{\mathrm{C}}$, $\bar{N}_{C} \pm \Delta \bar{N}_{C}$, where we have dropped the dependence on $\bar{E}_{n i}$ as only one neutron energy interval at a time need be considered. Let the integrated ion chamber readings for the two targets be $I_{o x}$ and $I_{c}$, and the masses of carbon in the two targets be $m_{o x}$ and $\mathrm{m}_{\mathrm{c}}$. Then the fraction of the neutrons incident on the polarimeter which came from the carbon in an oxalic acid target run is

$$
k_{c}=\frac{\bar{N}_{C} I_{o x} m_{o x}}{\bar{N}_{o x}{ }_{c} m_{c}}
$$

We can then subtract the carbon component of $\mathrm{P}_{\mathrm{Ox}}$ to obtain 


$$
\begin{aligned}
& P_{0} 16=\frac{\bar{P}_{o x}-k_{c} \bar{P}_{c}}{1-k_{c}} \\
& N_{0} 16=\bar{N}_{o x}\left(1-k_{c}\right)
\end{aligned}
$$

By the same method used previously equations may be derived for the statistical (or uncorrelated) and systematic (or correlated) components of the uncertainties in $\mathrm{P}_{0} 16$ and $\mathrm{N}_{0} 16$. The statistical uncertainties in $\mathrm{P}_{0} 16$ and $\mathrm{N}_{0} 16$ arise from the. statistical uncertainties in the measured quantities in eqs. IV.34 and IV.35. while the systematic uncertainties arise from the previously described polarimeter-tunnel scattering correction uncertainties. The final equations for $\Delta^{\mathrm{P}} 0^{16}=\left[\left(\Delta_{\mathrm{P}} 1 \mathrm{C}_{\mathrm{st}}\right)^{2}+\left(\Delta \mathrm{P}_{0} 16_{\text {sys }}\right)^{2}\right]^{1 / 2}$ and $\Delta N_{0} 16=\left[\left(\Delta N_{0} 16_{s t}\right)^{2}+\left(\Delta N_{0} 16_{s y s}\right)^{2}\right] 1 / 2$ are cumbersome and are given in Appendi-x III.

The corrections for polarimeter and tunnel scattering, and background, were subtracted in two ways, these differing mainly in the order of their subtraction. The procedure described thus far involves first subtracting the corrections for polarimeter and tunnel scattering and then using the results of this calculation for background subtraction. The second procedure involved first subtracting the background, and then subtracting the corrections for polarimeter and tunnel scattering. The former method proved simpler to use for computations, but unecessarily introduced the systematic uncertainties involved in subtracting the corrections for polarimeter and tunnel scattering from the background run. The latter method avoided this problem, but involved handling more data as the background must be subtracted separately for each side-detector pair because of differences in the as-yet- 
not-made polarimeter and tunnel scattering corrections. In practice the first method was used when the background formed less than $10 \%$ of the neutron beam and its contribution to the uncertainties was less than $10 \%$. When analyzing data by the second method the corrections for polarimeter and tunnel scattering are made as shown in section $E$, except that the background has already been subtracted from each side-detector pair, with a corresponding increase in the statistical uncertainties.

\section{E. Subtraction of the Corrections Due to Scattering in the}

\section{Photoneutron Target}

Since the photoneutron targets generally had dimensions which were an appreciable fraction of a neutron mean free path; it was necessary to make corrections for the scattering of neutrons in the target. Photoneutrons may be produced at an. arbitrary angle and then scattered toward the polarimeter, thus affecting the measured polarization. The effect of the scattered neutrons must be calculated and the measured photoneutron polarization corrected for these effects. The details of the calculations will be given in chapter VI, with the present discussion only describing the use of the results of the calculations.

For a specified target, bremsstrahlung end point, and photoneutron production angle the calculation of the corrections due to scattering in the photoneutron target yield a set of polarizations and fractions, $P_{t s}\left(\bar{E}_{n i}\right)$ and $k_{t s}\left(\bar{E}_{n i}\right)$ which are a function of the energy interval of the neutron beam incident on the polarimeter, $\bar{E}_{n i} \cdot P_{t s}\left(\dot{\bar{E}}_{n i}\right)$ is the polarization of the 
component of the neutron beam which underwent scattering in the photoneutron target, while $k_{t s}\left(\bar{E}_{n i}\right)$ is the fraction of the beam incident on the polarimeter, in the interval $E_{n i}$, which these neutrons form.

The target scattering corrections are subtracted from the data resulting from the stage of analysis described in section D. After the polarimeter and tunnel scattering corrections, and background, have been subtracted we have the results $\mathrm{P}_{\mathrm{t}}$ and $N_{t}$, which are generalizations of the $0^{16}$ example, i.e. $\mathrm{P}_{0} 16$ and $\mathrm{N}_{0} 16$ of eqs. IV.35. The polarization results may be expressed as

$$
\begin{aligned}
\mathrm{P}_{\mathrm{t}} & \pm \mathrm{P}_{\mathrm{t}} \\
\Delta \mathrm{P}_{\mathrm{t}} & =\left[\left(\Delta \mathrm{P}_{\mathrm{tst}}\right)^{2}+\left(\Delta \mathrm{P}_{\mathrm{t} s y s}\right)^{2}\right] 1 / 2
\end{aligned}
$$

which then allows the target scattering corrections to be subtracted as in eq. IV.23, to give the final results

$$
P_{F}=\frac{P_{t}-k_{t s} P_{t s}}{1-k_{t s}}
$$

An error analysis, separating the statistical and systematic uncertainties, yields

$$
\begin{aligned}
\Delta \mathrm{P}_{\text {Fst }}= & \frac{\Delta \mathrm{P}_{\mathrm{tst}}}{1-\mathrm{k}_{\mathrm{ts}}} \\
\Delta \mathrm{P}_{\mathrm{Fsys}}= & {\left[\left(\frac{\mathrm{k} \mathrm{ts}}{1-\mathrm{kts}}\right)^{2}\left(\Delta_{\mathrm{ts}}\right)^{2}+\frac{1}{\left(1-\mathrm{k}_{\mathrm{ts}}\right)^{2}}\left(\Delta_{\mathrm{tsys}}\right)^{2}\right.} \\
& \left.+\left(\frac{\mathrm{P}_{\mathrm{t}}-\mathrm{P}_{\mathrm{ts}}}{\left(1-\mathrm{k}_{\mathrm{ts}}\right)^{2}}\right)^{2}\left(\Delta_{\mathrm{ts}}\right)^{2}\right] 1 / 2 \\
\Delta \mathrm{P}_{\mathrm{F}}= & {\left[\left(\mathrm{\Delta P}_{\mathrm{Fst}}\right)^{2}+\left(\Delta_{\mathrm{Fsys}}\right)^{2]} 1 / 2 .\right.}
\end{aligned}
$$

IV .38

where the target scattering correction uncertainties 
are assumed to be entirely systematic. The uncertainties in $P_{t s}$ and $k_{t s}$ are taken to be (see chapter VI)

$$
\begin{aligned}
\Delta \mathrm{P}_{\mathrm{ts}} & =0.3 \mathrm{P}_{\mathrm{ts}} \\
\Delta \mathrm{k}_{\mathrm{ts}} & =0.3 \mathrm{k}_{\mathrm{ts}}
\end{aligned}
$$

The results in eq. IV.37 are the final values for the polarization of the photoneutrons from a given target. The results come from the raw polarizations, with corrections for polarimeter and tunnel scattering, background neutrons, and corrections for internal scattering in the photoneutron target, all subtracted. 'The uncertainties in eqs. IV.38 are final except tor polarımeter misalıgnment effects which are discussed in section $F$. As will be shown in Section F, polarimeter misalignment effects make a negligible contribution to the uncertainties, so eqs. IV.38 give effectively the final results for the uncertainty in the polarizations. F. Effects of Polarimeter Misalignment

The final source of uncertainty in the polarization measurements to be discussed is that arising from misalignment of the polarimeter. There are two types of such misalignment. First, the gas chamber may be located asymmetrically in the neutron beam, and second, the side-detector plane may be slightly rotated about the vector $\vec{k}_{\gamma} \times \vec{k}_{n}$ so that the two side-detectors in a pair are not at the same central scattering angle. Rotation of the polarimeter about $\vec{k}_{n}$ by a few degrees or less has a negligible effect and will not be considered further. The first type of misalignment arises principally from 
the gas chamber not being located in the center of the neutron beam. The gas chamber position was checked against cross hairs on the neutron collimator with a transit located in the target room, and it is estimated that the placement error in the gas chamber was less than $1 / 8 "$. Since the neutron beam was collimated to about 6" diameter at the gas chamber with the penumbra bringing it effectively up to 7" diameter, and the gas volume is a 5" diameter cyclinder, this gives an effective displacement of the scattering He volume w.r.t. the side-detectors of about 0.016" to the right or to the left. This possible displacement introduces an uncertainty in the equality of the central scattering angles and the effective solid angles of the two side-detectors in a pair.

The second type of misalignment comes from several causes:

1) Improper positioning of the side-detectors because of incorrectly located holes for bolting the side-detectors to the aluminum mounting plate. Because the bolt holes and the 10" diameter holes for fitting the mounting plates around the gas chamber base are machined to $\pm 0.005^{\prime \prime}$ this produces a maximum error in the side-detector scattering angles of about 1 min. of arc.

2) The mirror and carpenter's level used with the transit to align the polarimeter may introduce some errors because of imperfections. Also, the reflected image of the transit scope may not be lined up with the scope's vertical cross hairs. The mirror was first surface and optically flat, while the level was checked for flatness to $0.001^{\prime \prime}$. Thus the contacting surfaces of the level and mirror may be non-parallel to the contact points of the level and the brass plugs in the mounting 
plate by about $0.001 "$ in $10^{\prime \prime}$, the width of the mirror, and this introduces an error of about $1 / 4 \mathrm{~min}$. of arc in the side-detector angles. The image of the transit scope was displaced from the vertical cross hairs by less than $1 / 4 "$, with the total light path being about $60 \mathrm{ft}$, which gives about 1 min. of arcalignment accuracy. Thus the total error in the optical alignment was about $1 \mathrm{~min}$. of arc in the side-detector angle.

3) The center of the photoneutron target was positioned to about 1/8". For the 45 degree data this gives about 2 min. of arc, and for the 90 degree data about 1 min. of arc uncertainty in the side-detector angle. The shift of the effective center of the target because of the attenuation of the bremsstrahlung beam in the target is negligible. For a $2 "$ thick target a $10 \%$ attenuation of the photon beam only shifts the effective center of neutron production by $1 / 50$ " from the true center-of-mass. This corresponds to about the worst case in actual targets used, and results in a negligible shift compared with the $1 / 8$ " uncertainty in true center-of-mass position.

Combining all the errors in angular misalignment gives an expected uncertainty of about $2 \mathrm{~min}$. of arc in the sidedetector angles.

The calculation of misalignment effects starts with eq. I I. 9 ,

$$
\begin{array}{ll}
N_{R}=N n_{R} \frac{d \sigma}{d \Omega}(\theta 0)\left[1+P_{n} P_{H e}\left(\theta^{\circ}\right)\right] & \\
N_{L}=N n_{L} \frac{d \sigma}{d \Omega}\left(\theta_{0}\right)\left[1-P_{n} P_{H e} e_{0}^{(\theta)}\right] & \text { IV. } 40
\end{array}
$$

If we consider a small shift $\delta$ of the gas volume to the right 
of, and perpendicular to, the neutron beam, then to first order in $\delta$ and $\Delta \theta=-\frac{\delta}{r_{0}} \cos \theta_{0}$

$$
\left.\left.\left.N_{(}^{R}\right)=N_{(}^{R}\right)\left(\theta_{L}^{R}\right)+\Delta N_{(}^{R}\right)
$$

where

$$
\Delta N_{\left(\begin{array}{l}
R \\
L
\end{array}\right)}= \pm N\left(\begin{array}{l}
R \\
L
\end{array}\right)\left(\theta_{0}\right) \quad\left[\left.\left[\frac{\frac{\partial}{\theta}\left(\frac{d \sigma}{d \Omega}\right)}{\left(\frac{d \sigma}{d \Omega}\right)}+\frac{P_{n}-\frac{\partial P_{H e}}{\partial \theta}}{1 \pm P_{n} P_{H e}}\right]\right|_{\theta=\theta_{0}}+\frac{\left.2 \delta \sin \theta_{\theta}\right]}{r_{0}}\right]
$$

In eq. IV.42, $\theta_{0}$ is the central scattering angle for the sidedetector pair in question and $r_{0}$ is the center-to-center distance of the gas chamber to side-detector. The derivation of eq.IV.42 takes account of the variation of $\eta_{(}^{\left(\frac{R}{L}\right)}$ with $\delta$ because the solid angle of the side-detectors changes with $\mathrm{r}_{0}$. Eq. IV.42 also holds for a rotation of the side-detectors by $\Delta \theta$, if $\delta$ is set to zero.

From eq. IV. 42 it can be shown that

$$
\begin{aligned}
A_{n} & =\frac{N_{R}-N_{L}}{N_{R}+N_{L}} \\
& =A_{n}\left(\theta_{0}\right)+\Delta A_{n}
\end{aligned}
$$

where

$$
\begin{aligned}
& A_{n}\left(\theta_{0}\right)=\frac{N_{R}\left(\theta_{0}\right)-N_{L}\left(\theta_{0}\right)}{N_{R}\left(\theta_{0}\right)+N_{L}\left(\theta_{0}\right)} \\
& \Delta A_{n} \simeq\left[\frac{2 \delta \sin \theta_{0}}{r_{0}}+\left.\left[\frac{\left[\frac{\partial}{\partial \theta}\left(\frac{d \sigma}{d \Omega}\right)\right.}{\left(\frac{d \sigma}{d \Omega}\right)}+P_{n\left(-\frac{\partial P_{2}}{\partial \theta}\right)}\right]\right|_{\left.\theta=\theta_{0}\right]}\right]
\end{aligned}
$$

In deriving eq. IV.44 the denominator $1 \pm{ }_{P_{n}} P_{H e}$ of eq. IV.42 has been set equal to unity.

The estimates of $\Delta A_{n}$ must be made with $\delta \simeq 0.016^{\prime \prime}$, and 
with $\Delta \theta \simeq 2$ min. of arc. With $\delta=0.016^{\prime \prime} \mathrm{a} \Delta \theta=-\frac{\delta}{r_{0}} \cos \theta_{0}$ in eq. IV.44 is also generated. The results of eq. IV.44 for $\delta=0.016^{\prime \prime}$, $\Delta \theta=-\frac{\delta}{r_{0}} \cos \theta 0$, are shown in table IV-2 for all side-detector angles used, with $\mathrm{r}_{\mathrm{o}}=20^{\prime \prime}$ or $30^{\prime \prime}$ depending on the angle, and for incident neutron energies of $3,6,10$ and $14 \mathrm{MeV}$. Table IV-3 also shows the results of eq. IV.44 for $\Delta \theta=2 \mathrm{~min}$. of arc when $\delta=0$. The results have been calculated with $\mathrm{P}_{\mathrm{n}}= \pm 0.5$, whichever value gave the larger $\left|\Delta A_{n}\right|$.

Experimental tests of misalignment effects were made with 14.7 MeV neutrons from the $t(d, n)$ reaction. Since the zero degree produced neutrons are expected to have zero polarization, measurements were made on the forward beam with the polarimeter properly aligned, with the side-detectors misaligned by 2 degrees $\left(\delta=0, \Delta \theta=-2^{\circ}\right)$, and with the entire polarimter displaced $1^{\prime \prime}$ perpendicular to the neutron beam $\left(\delta=-l^{\prime \prime}, \Delta \theta=\frac{\delta}{r_{0}} \cos \theta 0\right)$. The measurements were made with $r_{0}=30^{\prime \prime}, \theta_{0}=113$ and 155 degrees. The results for $A_{n}\left(=\Delta A_{n}\right)$ are given in table IV-4 along with the calculated values from eq. IV.44. The results of experiments are in reasonable agreement with the calculations, so eq. IV.44 can be used to estimate the uncertainties arising from polarimeter misalignment.

The values of $\Delta A_{n}$ shown in tables $I V-2$ and $I V-3$ indicate that uncertainties from misalignment may be neglected compared with the statistical and systematic uncertainties calculated as described earlier in this chapter. The maximum value of $\Delta A_{n}$ is 0.0028 for $r_{0}=20^{\prime \prime}$ and 0.0017 for $r_{0}=30^{\prime \prime}$. When divided by the appropriate average of $\overline{\mathrm{P}}_{\mathrm{He}}\left(\theta \circ, \overline{\mathrm{E}}_{\mathrm{n}}\right)(\mathrm{eq}$. IV.12), the resulting values of $\Delta \mathrm{P}_{n}$ are negligible compared with the uncertainties 
calculated with eq. IV. 38 which accounts for statistical errors and uncertainties in the various corrections. Thus the results for $P_{F} \pm \Delta P_{F}$, the measured and corrected neutron polarization and its uncertainty, are adequately given by eqs. IV.37 and IV.38 which allow only for statistical errors in the uncorrected asymmetries, and errors arising from imprecise knowledge of the corrections for scattering by the polarimeter and tunnel and for internal scattering in the photoneutron target. 


\begin{tabular}{|c|c|c|c|c|c|c|c|c|c|c|}
\hline $\begin{array}{l}\delta=.016^{\prime \prime} \\
\mathrm{r}_{\mathrm{o}}=20^{\prime \prime} \\
\mathrm{E}_{\mathrm{n}} ; \mathrm{MeV}\end{array}$ & \multicolumn{2}{|c|}{$\theta 0=47^{\circ}$} & \multicolumn{2}{|c|}{$\theta_{0}=67^{\circ}$} & \multicolumn{2}{|c|}{$\theta_{0}=88^{\circ}$} & \multicolumn{2}{|c|}{$\theta_{0}=117^{\circ}$} & \multicolumn{2}{|c|}{$\theta_{0}=137^{\circ}$} \\
\hline 3 & -.0018 & +.5 & -.0021 & -.5 & -.0017 & -.5 & -.0016 & +.5 & -.0014 & -.5 \\
\hline $6^{\circ}$ & -.0022 & +.5 & -.0022 & +.5 & -.0017 & -.5 & -.0018 & -.5 & -.0019 & -.5 \\
\hline 10 & -.0024 & +.5 & -.0025 & +.5 & -.0018 & -.5 & -.0023 & -.5 & -.0022 & -.5 \\
\hline 14 & -.0025 & +.5 & -.0026 & +.5 & -.0018 & -.5 & -.0025 & -.5 & -.0022 & -.5 \\
\hline
\end{tabular}

TABLE IV-2

\begin{tabular}{|c|c|c|c|c|c|c|c|c|}
\hline $\begin{aligned} \delta & =.016^{\prime \prime} \\
\mathrm{r}_{\mathrm{O}} & =30^{\prime \prime} \\
\mathrm{E}_{\mathrm{n}}, & \mathrm{MeV}\end{aligned}$ & $\begin{array}{c}\theta_{0}= \\
\Delta \mathrm{A}_{\mathrm{n}}\end{array}$ & $\mathrm{P}_{\mathrm{n}}$ & $\begin{array}{l}\theta_{0}= \\
\Delta A_{n}\end{array}$ & $P_{n}$ & $\Delta \mathrm{A}_{\mathrm{n}}$ & $141^{\circ}$ & $\Delta A_{n}$ & $\begin{array}{c}155^{\circ} \\
\mathrm{P}_{\mathrm{n}}\end{array}$ \\
\hline 3 & -.0011 & +.5 & -.0011 & -.5 & -.0011 & -.5 & -.0009 & -.5 \\
\hline 6 & -.0012 & +.5 & -.0013 & -.5 & -.0012 & -.5 & -.0010 & -.5 \\
\hline 10 & -.0014 & +.5 & -.0016 & -.5 & -.0014 & -.5 & -.0011 & -.5 \\
\hline 14 & -.0016 & -.5 & -.0017 & -.5 & -.0014 & -.5 & -.0010 & -.5 \\
\hline
\end{tabular}




\begin{tabular}{|c|c|c|c|c|c|c|c|c|c|c|}
\hline \multirow{2}{*}{$\frac{\Delta \theta=2^{\prime}}{\mathrm{E}_{\mathrm{n}}, \mathrm{MeV}}$} & \multicolumn{2}{|c|}{$\begin{array}{c}\theta_{0}=47^{\circ} \\
\Delta A_{n} \cdot P_{n}\end{array}$} & \multicolumn{2}{|c|}{$\begin{array}{c}\theta_{0}=67^{\circ} \\
\Delta A_{n} \quad P_{n}\end{array}$} & \multicolumn{2}{|c|}{$\begin{array}{c}\theta_{0}=88^{\circ} \\
\mathrm{A}_{\mathrm{n}} \quad \mathrm{P} \\
{ }_{\mathrm{n}}\end{array}$} & \multicolumn{2}{|c|}{$\begin{array}{c}\theta_{0}=117^{\circ} \\
\Delta \mathrm{A}_{\mathrm{n}} \quad \mathrm{P}_{\mathrm{n}}\end{array}$} & \multicolumn{2}{|c|}{$\begin{array}{l}\theta_{0}=137^{\circ} \\
\Delta A_{n} \cdot P_{n}\end{array}$} \\
\hline & -.0007 & +.5 & -.0011 & -.5 & -.0012 & -.5 & +.0002 & +.5 & +.0005 & -.5 \\
\hline 6 & -.0011 & +.5 & $\therefore .0012$ & +.5 & -.0017 & -.5 & +.0006 & -.5 & +.0008 & -.5 \\
\hline 10 & -.0012 & +.5 & -.0019 & +.5 & -.0025 & -.5 & +.0014 & -.5 & +.0011 & -.5 \\
\hline 14 & -.0013 & +.5 & -.0020 & +.5 & -.0028 & -.5 & +.0017 & -.5 & +.0011 & -.5 \\
\hline
\end{tabular}

\section{TABLE IV -3}

\begin{tabular}{|c|c|c|c|c|c|c|c|c|}
\hline $\begin{array}{c}\Delta \theta=2^{\prime} \\
\mathrm{E}_{\mathrm{n}}, \mathrm{MeV}\end{array}$ & \multicolumn{2}{|c|}{$\theta_{0}=113^{\circ}$} & \multicolumn{2}{c|}{$\theta_{0}=127^{\circ}$} & \multicolumn{2}{|c|}{$\theta_{0}=141^{\circ}$} & \multicolumn{2}{|c|}{$\theta_{0}=155^{\circ}$} \\
\hline 3 & +.0003 & +.5 & +.0004 & -.5 & +.0006 & -.5 & +.0004 & -.5 \\
6 & +.0007 & +.5 & +.0008 & -.5 & +.0007 & -.5 & +.0006 & -.5 \\
10 & +.0012 & +.5 & +.0014 & -.5 & +.0010 & -.5 & +.0006 & -.5 \\
14 & +.0017 & -.5 & +.0015 & -.5 & +0010 & -.5 & +.0006 & -.5 \\
\hline
\end{tabular}


14.7 MeV neutron beam, zero polarization

\begin{tabular}{|c|c|c|}
\hline \multirow[b]{2}{*}{$\begin{array}{l}\text { Proper Alignment, } \\
\text { measured } A_{n}\end{array}$} & $113^{\circ}$ & $155^{\circ}$ \\
\hline & $-.013 \pm .007$ & $+.010 \pm .005$ \\
\hline $\begin{aligned} \text { Displaced } 1^{\prime \prime} & - \text { experimental } A_{n} \\
& - \text { calculated } A_{n}\end{aligned}$ & $\begin{array}{l}-.026 \pm .008 \\
-.020\end{array}$ & $\begin{array}{l}-.014^{+} .005 \\
-.011\end{array}$ \\
\hline $\begin{aligned} \text { Rotated } 2^{\prime \prime} & - \text { experimental } A_{n} \\
& - \text { calculated } A_{n}\end{aligned}$ & $\begin{array}{l}-.054 \pm .007 \\
-.098 \\
.\end{array}$ & $\begin{array}{l}-.010 \pm .005 \\
-.024\end{array}$ \\
\hline
\end{tabular}

$\underline{T A B L E} \cdot \mathrm{IV}-4$ 


\section{CHAPTER V}

\section{RESULTS}

The results of the photoneutron polarization measurements are plotted in figs. V-1 to $\mathrm{V}-24$. Table $\mathrm{V}-1$ is a tabulation of the plots according to desired element, photoneutron production angle, and bremsstrahlung end-point energy. For each combination of element, production angle, and bremsstrahlung energy the results for each of three stages of data analysis are plotted for most of the actual targets used. The last plot for each group shows the averaged final results for a given element, photoneutron production angle, and bremsstrahlung energy. A11 plots show neutron polarization vs. neutron energy, while the plots of the final results also have a second energy scale showing the ground state transition photon energies for photoneutron production on the desired element.

The three stages of data analysis plotted are: a) the uncorrected polarization, b) the polarization after background has been subtracted, and c) the polarization after background has been subtracted and corrections made for polarimeter and tunnel scattering. The order in which this analysis was performed is shown by the numbers, appearing in the appropriate columns of table V-1. The following is a more detailed commentary on the results 1 isted in table $V-1$.

A. $0^{16}, 45$ degrees, $24 \mathrm{MeV}$ bremsstrahilung

These results were derived from four runs using an oxalic acid target, and one $B$ run using a water target. The background run was made with a polyethylene cyclinder for a target. 
runs were made with the stainless steel gas chamber. The results for the oxalic acid runs are plotted in fig. V-l, in the three stages of analysis indicated in table V-1. The run with a water target was short and had poor statistical errors, and is only included in the final results in fig. V-2.

The plots in fig. V-1 show that the corrections for polarimeter and tunnel scattering produce the largest effect for neutrons in the range $2-5 \mathrm{MeV}$. This arises because the $0^{16}$ $(\gamma, n)$ giant resonance peak is in the neutron energy range of $6-8 \mathrm{MeV}$. An $\mathrm{O}^{16}$ bremsstrahlung photoneutron spectrum thus has more neutrons in the 6-8 MeV range compared to the 2-5 MeV range, and the corrections for scattering by the 6-8 MeV neutrons tend to wash out the polarization measurements on the lower energy neutrons.

B. $0^{16}, 45$ degrees, $30 \mathrm{MeV}$ bremsstrahlung

These results were derived from five runs with a water target and four runs with liquid oxygen targets, all taken with the stainless steel gas chamber, and two runs with oxalic acid taken with the aluminum gas chamber. The background runs (Iisted in table III-1, of chapter III) were: one run with an empty polyethylene bottle for the water runs; one run with an empty styrofoam dewar for three of the liquid oxygen runs; one run with an empty mylar target holder for the fourth liquid oxygen run; and a run with a spherical carbon shell target for the oxalic acid runs.

Fig. V-3 shows three plots of the data from three of the water runs: the uncorrected results; with the background subtracted; and with background subtracted and corrections made 
for scattering by the polarimeter and tunnel. Fig. V-4 shows the same three stages of analysis for two of the liquid oxygen runs with the styrofoam dewar. These two figures illustrate the large scattering corrections for the stainless steel gas chamber, especially in the region of $2-5^{-} \mathrm{MeV}$ neutron energy, where errors become quite large after the corrections have been made. Some of the low energy points have been left out of the final plots because of error bars so large as to render the data meaningless.

Fig. V-5 shows three plots for the oxalic acid runs made with the aluminum gas chamber. The first plot is the uncorrected data, the second shows the data after corrections have been made for polarimeter and tunnel scattering, and the third shows the data after making corrections for polarimeter and tunnel scattering and subtracting background. The smaller polarimeter scattering corrections with the aluminum gas chamber are obvious by comparing these plots with figs. V-3 and V-4. However, the aluminum gas chamber gives poorer energy resolution than the stainless stee1 chamber.

The final results after making corrections for scattering within the photoneutron targets and adding all the data together are shown in fig. V-6.

C. $0^{16}, 45$ degrees, 38 MeV bremsstrahlung

These results were derived from four runs with a water target. Background was taken from a $30 \mathrm{MeV}$ run on an empty polyethylene bottle. Fig. V-7 shows plots of the raw data, after background subtraction, and after background subtraction and correction for scattering by the polarimeter and tunnel. The final results, including corrections for scattering within the photoneutron target are plotted in fig. V- 8 . The $38 \mathrm{MeV}$ data 
were analyzed in $1 / 2 \mathrm{MeV}$ neutron energy intervals to look for structure in the polarization.

D. $0^{16}, 90$ degrees, $30 \mathrm{MeV}$ bremsstrahlung

These results were derived from four runs with a water target, and two runs with a spherical shell of oxalic acid for a target. The background for the water runs was a single run with an empty polyethylene bottle target, while for the oxalic acid runs it was calculated from the $\mathrm{C}^{12}$ and $\mathrm{O}^{16}$ photoneutron cross sections. The water runs were made with the stainless steel gas chamber while the oxalic acid runs were made with the aluminum gas chamber.

Fig. V-9 shows plots of the water data, first with no corrections, second with corrections made for scattering by the polarimeter and tunnel, and third with corrections made for scattering by the polarimeter and tunnel and the experimental background subtracted. Fig. V-10 shows the same sequence of three plots for the oxalic acid runs. The background for the oxalic acid runs was calculated from the $\mathrm{C}^{12}$ and $\mathrm{O}^{16}$ photoneutron cross section measurements of Verbinski and Courtney . The averaged final results, corrected for scattering within the photoneutron target, are plotted in fig. V-11. A theoretical discussion of the $0^{16}$ results is given in Chapter VII.

E. $\mathrm{C}^{12}, 45$ degrees, $30 \mathrm{MeV}$ bremsstrahlung

These results were derived from two $30 \mathrm{MeV}$ runs on a spherical carbon shell target, taken with the stainless steel gas chamber. A third run was made with the carbon shell and the aluminum gas chamber. Figs. V-12 and V-13 show the two results, first uncorrected, and second with corrections made for scattering by the polarimeter and tunnel. The average of the two sets, with 
corrections made for scattering within the photoneutron target, forms the final results shown in fig. $\mathrm{V}-14$.

F. $\mathrm{C}^{12}, 45$ degrees, $47 \mathrm{MeV}$ bremsstrahlung

These results were derived from 9 runs with a target of a spherical shell of graphite and one run with a spherical shell of polyethylene. All runs were made with the aluminum gas chamber. Fig. V-15 shows the results for four of the carbon runs before and after making corrections for scattering by the polarimeter and tunnel. Fig. V-16 shows the final results, including corrections for scattering within the photoneutron target, averaged over all runs.

G. $\mathrm{C}^{12}, 90$ degrees, $50 \mathrm{MeV}$ bremsstrahlung

These results were derived from one run with a spherical carbon shell target, taken with the stainless steel gas chamber. Fig. V-17 shows the uncorrected results, and the results after making corrections for scattering by the polarimeter and tunnel. Fig. V-18 shows the final results after also making corrections for scattering within the photoneutron target. The results in fig. V-18 are averaged over larger neutron energy intervals than those in fig. V-17.

The $\mathrm{C}^{12}$ polarization at 90 degrees to a 30 MeV bremsstrahlung beam, as measured by S. Kowalski , is also plotted in fig. V-18. These results indicate that $\mathrm{C}^{12}$ has a polariation near zero for photoneutron production at 90 degrees. A theoretical interpretation of these $\mathrm{C}^{12}$ measurements will be given in Chapter VII.

H. $\mathrm{Ca}^{40}, 90$ degrees, $28 \mathrm{MeV}$ bremsstrahlung

These results were derived from one run made with the aluminum gas chamber. Fig. V-'19 shows the uncorrected data 
and the data corrected for scattering.by the polarimeter and tunnel. Fig. V-20 shows the final results after making corrections for scattering within the photoneutron target.

I. $\mathrm{Pb}^{208}, 45$ degrees, $28 \mathrm{MeV}$ bremsstrahlung

These results were derived from two runs made with the stainless steel gas chamber. Fig. V-21 shows the uncorrected data, and the data corrected for scattering by the polarimeter and tunnel.

The large errors for low.energy neutrons are mostly statistical because of the large random counting rate from the high flux of low energy neutrons.

Fig. V-22 shows the final results after making corrections for scattering within the photoneutron target.

J. $\mathrm{Pb}^{208}, 90$ degrees, $21 \mathrm{MeV}$ bremsstrahlung

These results were derived from two runs made with the stainless steel chamber. Fig. V-23 shows the uncorrected data, and the data corrected for scattering by the polarimeter and tunne1. Fig. V-24 shows the final results, with the data corrected for'scattering within the photoneutron target. 


\begin{tabular}{|c|c|c|c|c|c|c|c|c|c|}
\hline \multirow{2}{*}{$\begin{array}{l}\text { DESIRED } \\
\text { ELEMENT }\end{array}$} & \multirow{2}{*}{$\begin{array}{l}\text { Neutron } \\
\text { Production } \\
\text { Angle (lab), Deg: }\end{array}$} & \multirow{2}{*}{$\begin{array}{l}\text { Brems. } \\
\text { End-Point. } \\
\text { yev }\end{array}$} & \multirow{2}{*}{$\begin{array}{l}\text { Target } \\
\text { Used }\end{array}$} & \multirow{2}{*}{$\begin{array}{l}\text { Cias* } \\
\text { Chamber }\end{array}$} & \multirow{2}{*}{$\begin{array}{l}\text { Graph } \\
\text { Number }\end{array}$} & \multicolumn{3}{|c|}{$\begin{array}{l}\text { nrder of Data Analvsis } \\
\text { And Graphic Nisplay of Results }\end{array}$} & \multirow{2}{*}{$\begin{array}{c}\text { FINAL } \\
\text { RESULTS } \\
\text { Inc. Target } \\
\text { Scat. Corr. }\end{array}$} \\
\hline & & & & & & \begin{tabular}{|l|} 
Raw \\
Polarization \\
\end{tabular} & $\begin{array}{l}\text { Rackeround } \\
\text { Suhtracted } \\
\end{array}$ & $\begin{array}{l}\text { Pol. Tunnel } \\
\text { Scat. Corr. }\end{array}$ & \\
\hline \multirow[t]{4}{*}{$0^{16}$} & \multirow[t]{3}{*}{45} & $\therefore$ & $\begin{array}{l}\text { Oxalic } \\
\text { Acid } \\
\text { Water }\end{array}$ & $\begin{array}{l}\text { ss } \\
\text { ss }\end{array}$ & $\begin{array}{c}v-1 \\
v-2\end{array}$ & 1 & 2 & 3 & $x$ \\
\hline & & 30 & $\left\{\begin{array}{l}\text { water } \\
\text { Liquid } \\
\text { Oxygen } \\
\text { (styrf. } \\
\text { dwr. } \\
\text { Oxalic } \\
\text { Acid }\end{array}\right.$ & $\begin{array}{l}\text { SS } \\
\text { SS } \\
\text { Al. }\end{array}$ & $\begin{array}{l}v-3 \\
v-4 \\
v-5 \\
v-6\end{array}$ & $\begin{array}{l}1 \\
1 \\
1\end{array}$ & $\begin{array}{l}2 \\
2 \\
\\
3\end{array}$ & $\begin{array}{l}3 \\
3 \\
2\end{array}$ & $x$ \\
\hline & & 38 & Water & ss & $\begin{array}{l}V-7 \\
V-8\end{array}$ & 1 & 2 & 3 & $x$ \\
\hline & 90 & 30 & $\begin{array}{l}\text { Water } \\
\text { Oxalic } \\
\text { Acid }\end{array}$ & $\begin{array}{l}\text { SS } \\
\text { AI. }\end{array}$ & $\begin{array}{l}v-9 \\
v-10 \\
v-11\end{array}$ & $\begin{array}{l}1 \\
1\end{array}$ & $\begin{array}{l}3 \\
3\end{array}$ & $\begin{array}{l}2 \\
2\end{array}$ & $x$ \\
\hline \multirow[t]{3}{*}{$c^{12}$} & \multirow[t]{2}{*}{45} & 30 & $\begin{array}{l}\text { Carbon } \\
\text { Carbon }\end{array}$ & $\begin{array}{l}\text { SS } \\
\mathrm{Al} \text {. }\end{array}$ & $\begin{array}{l}v-12 \\
v-13 \\
v-14 \\
\end{array}$ & $\begin{array}{l}1 \\
1\end{array}$ & - & 2 & $x$ \\
\hline & & 47 & Carbon & Al. & $\begin{array}{l}v-15 \\
v-16 \\
\end{array}$ & 1 & - & 2 & $x$ \\
\hline & 90 & 50 & Carhon & ss & $\begin{array}{l}v-17 \\
v-18\end{array}$ & 1 & - & 2 & $x$ \\
\hline $\mathrm{Ca}^{40^{-}}$ & 90 & 28 & Calcium & $A L$ & $\begin{array}{l}v-19 \\
v-20\end{array}$ & 1 & - & 2 & $x$ \\
\hline \multirow[t]{2}{*}{$P_{b}^{208}$} & 45 & 28 & $\mathrm{P}_{\mathrm{b}}{ }^{208 * *}$ & sS & $\begin{array}{l}v-21 \\
v-22 \\
\end{array}$ & 1 & . & 2 & $x$ \\
\hline & 90 & 21 & $P_{b}^{208 * *}$ & SS & $\begin{array}{l}V-23 \\
V-24\end{array}$ & 1 & - &. & $x$ \\
\hline
\end{tabular}

* SS EStainless Steel Gas Chamber

AL ミNluminum lias Chamber

TABLE V-i

** About 958 P 208 target on loan from Dak Ridge
National Laboratory.

Tabulation of the Plots of the Photoneutron Polarization Results 


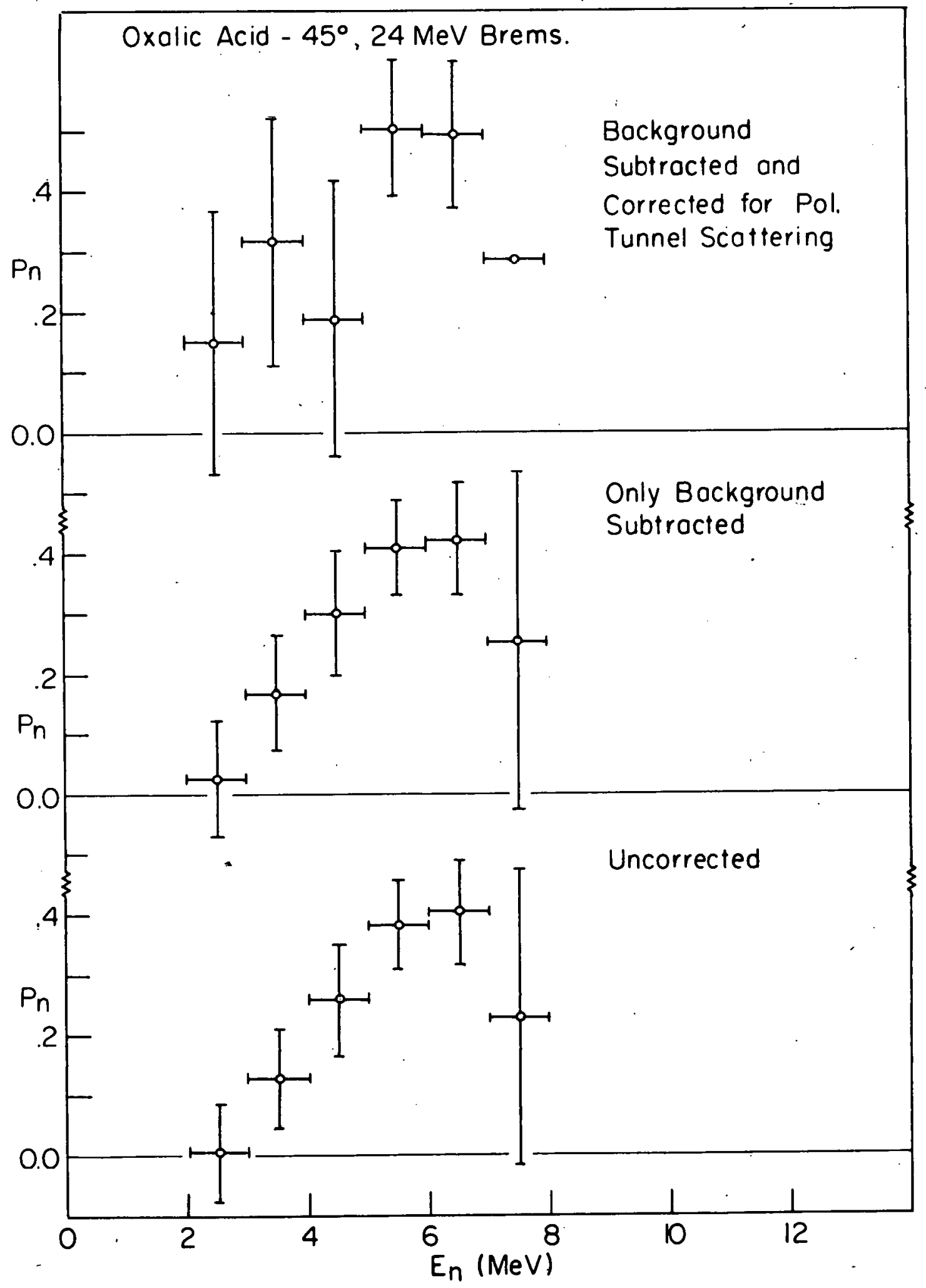

Fig. V-1 


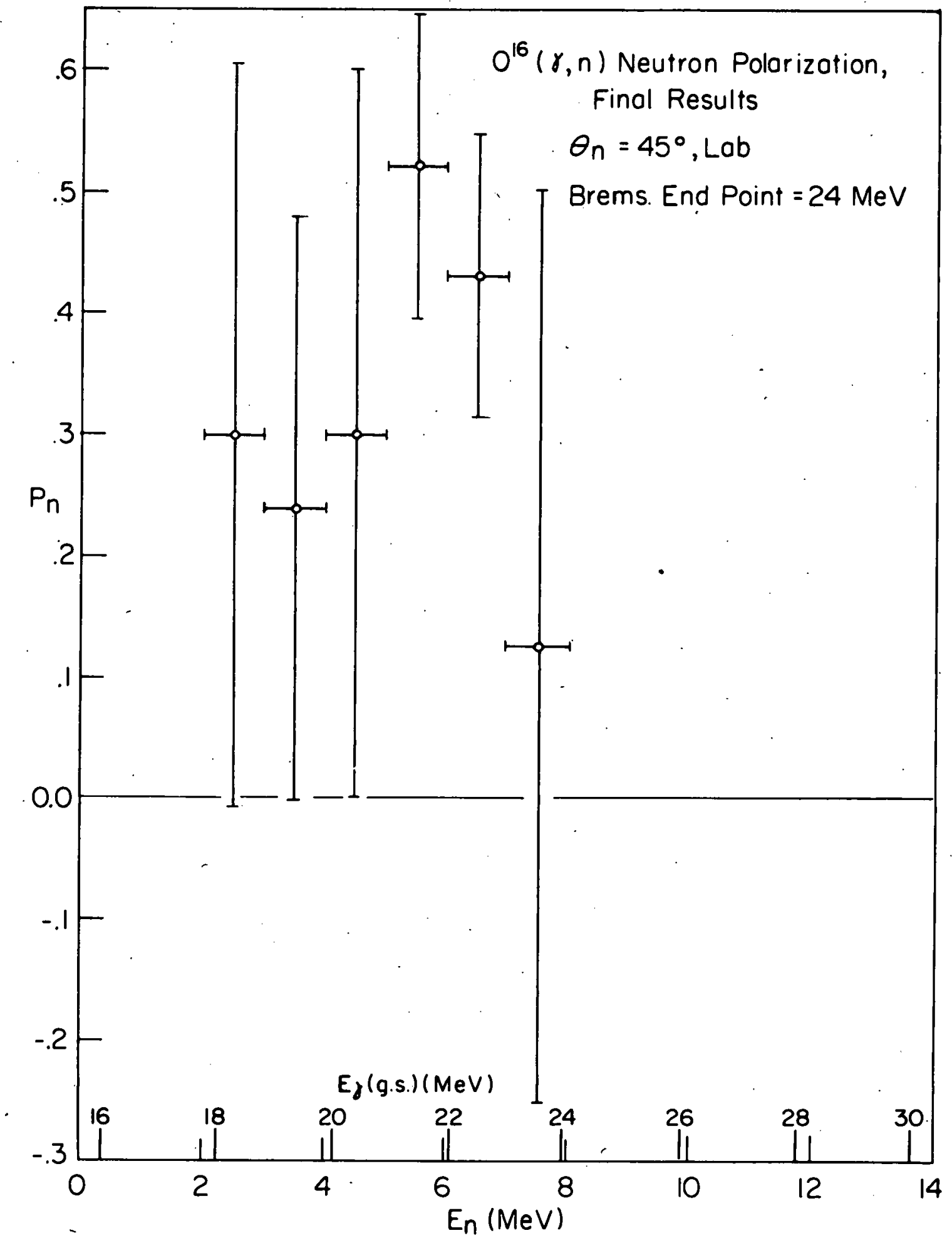

Fig. V-2 


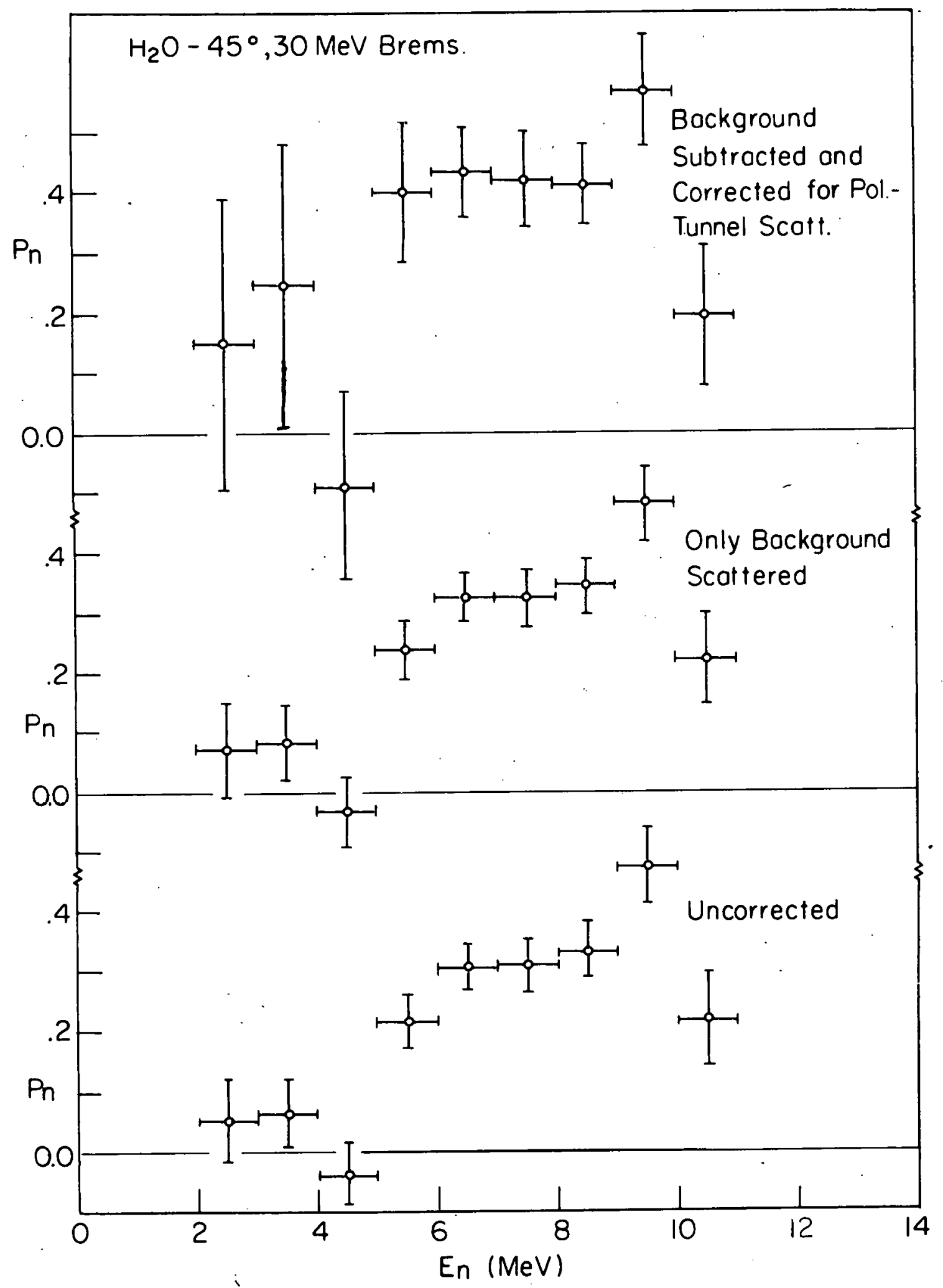

Fig. V-3 


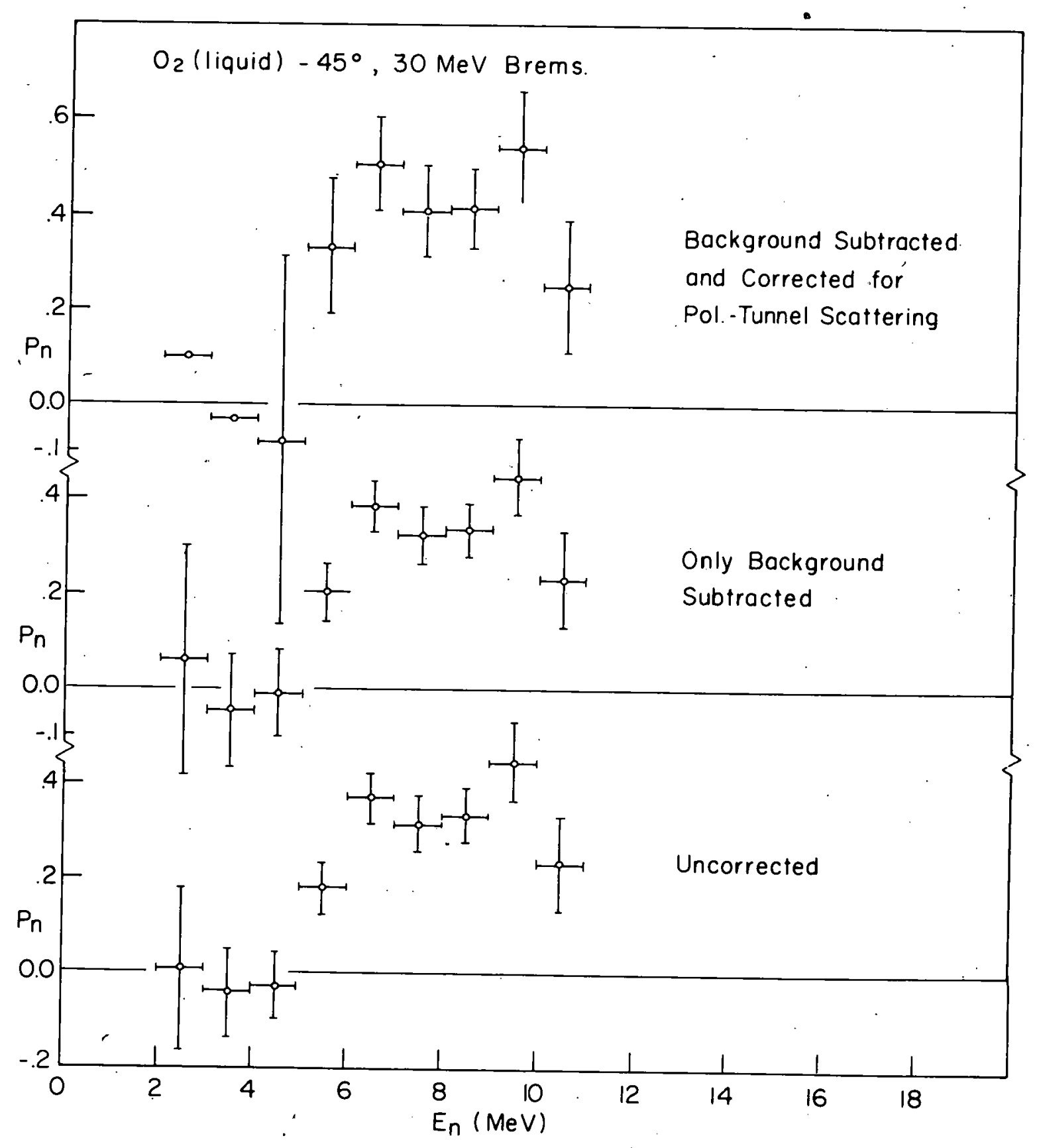

Fig. V-4 


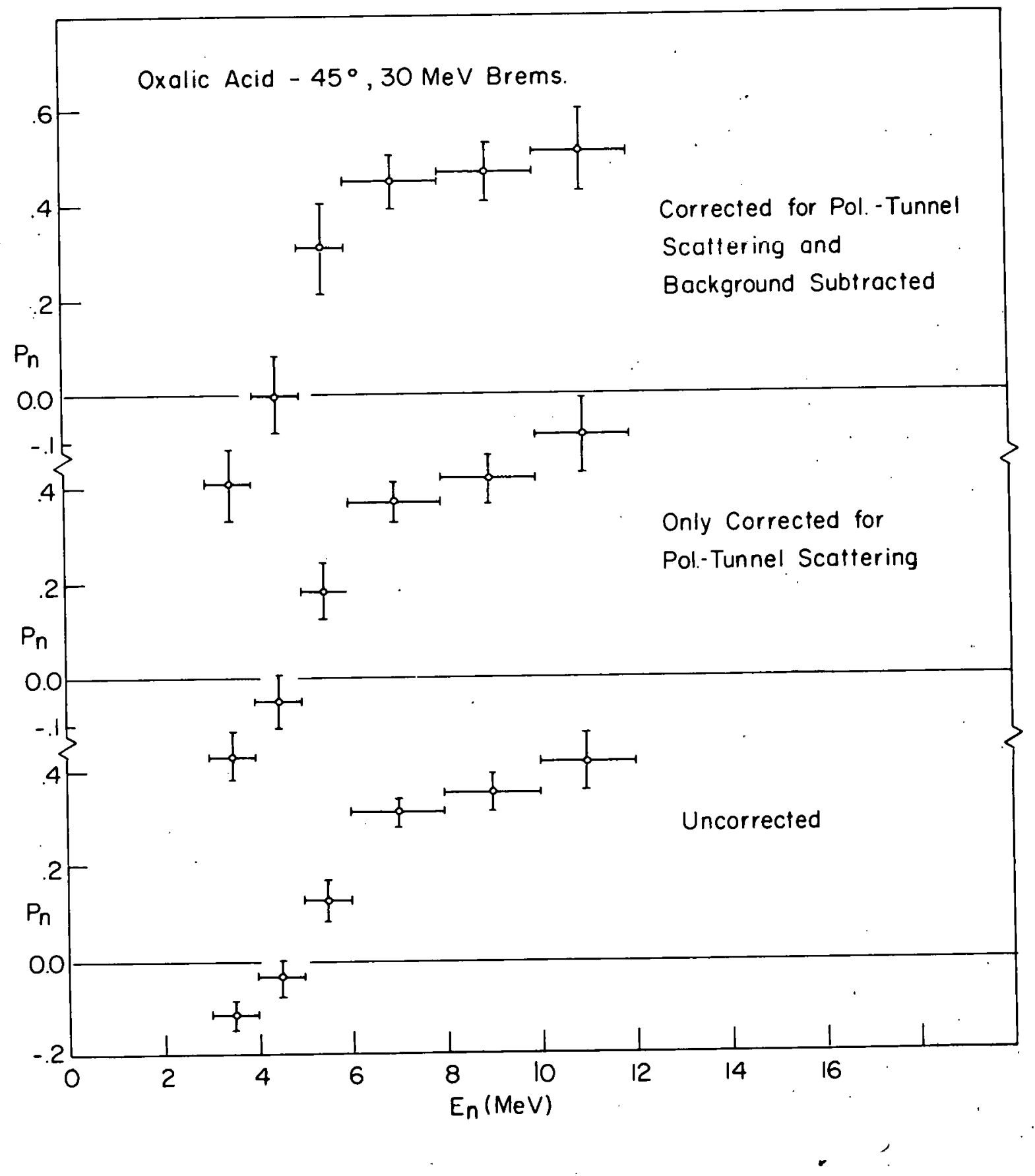

Fig. V-5 
$-125-$

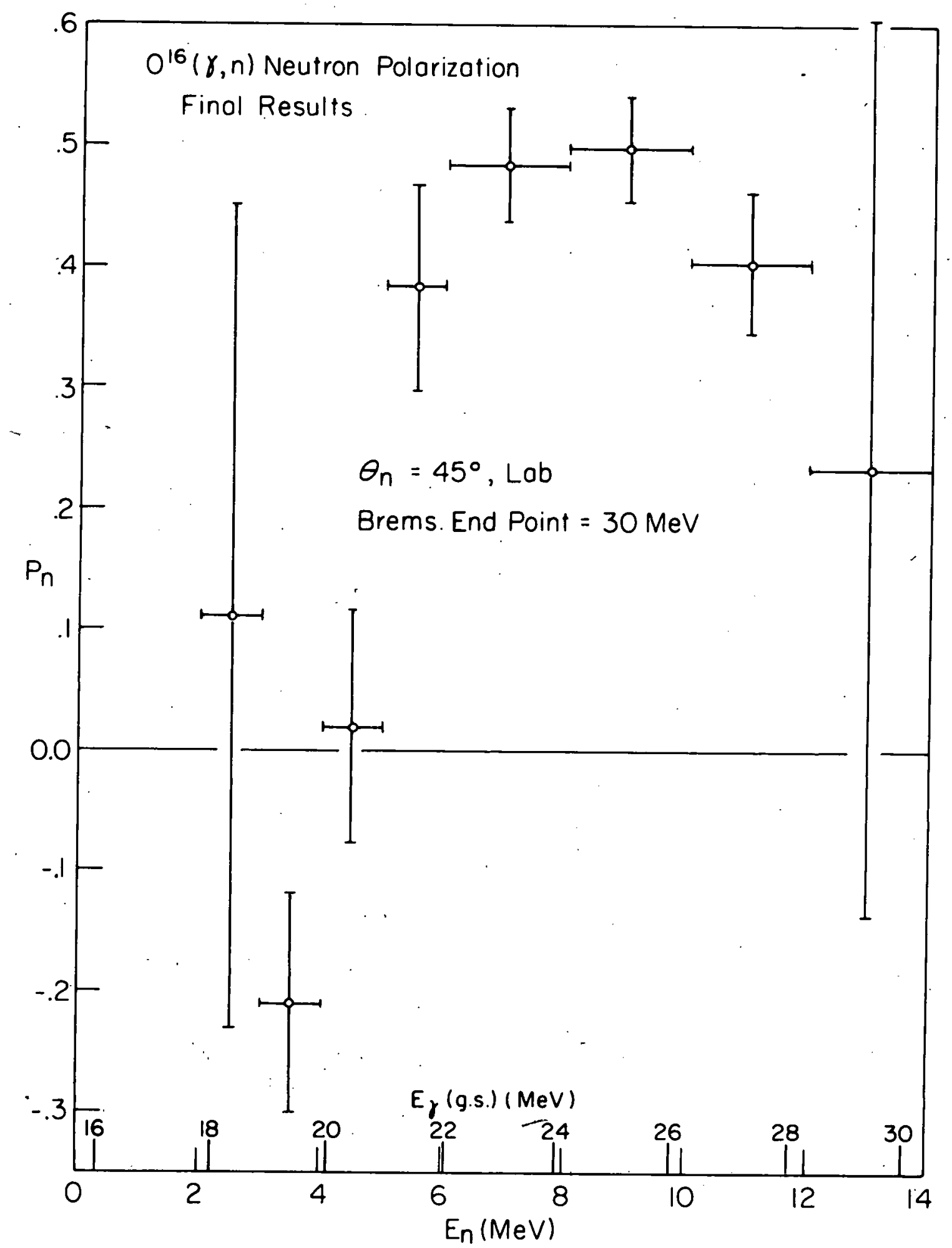

Fig. V-6 


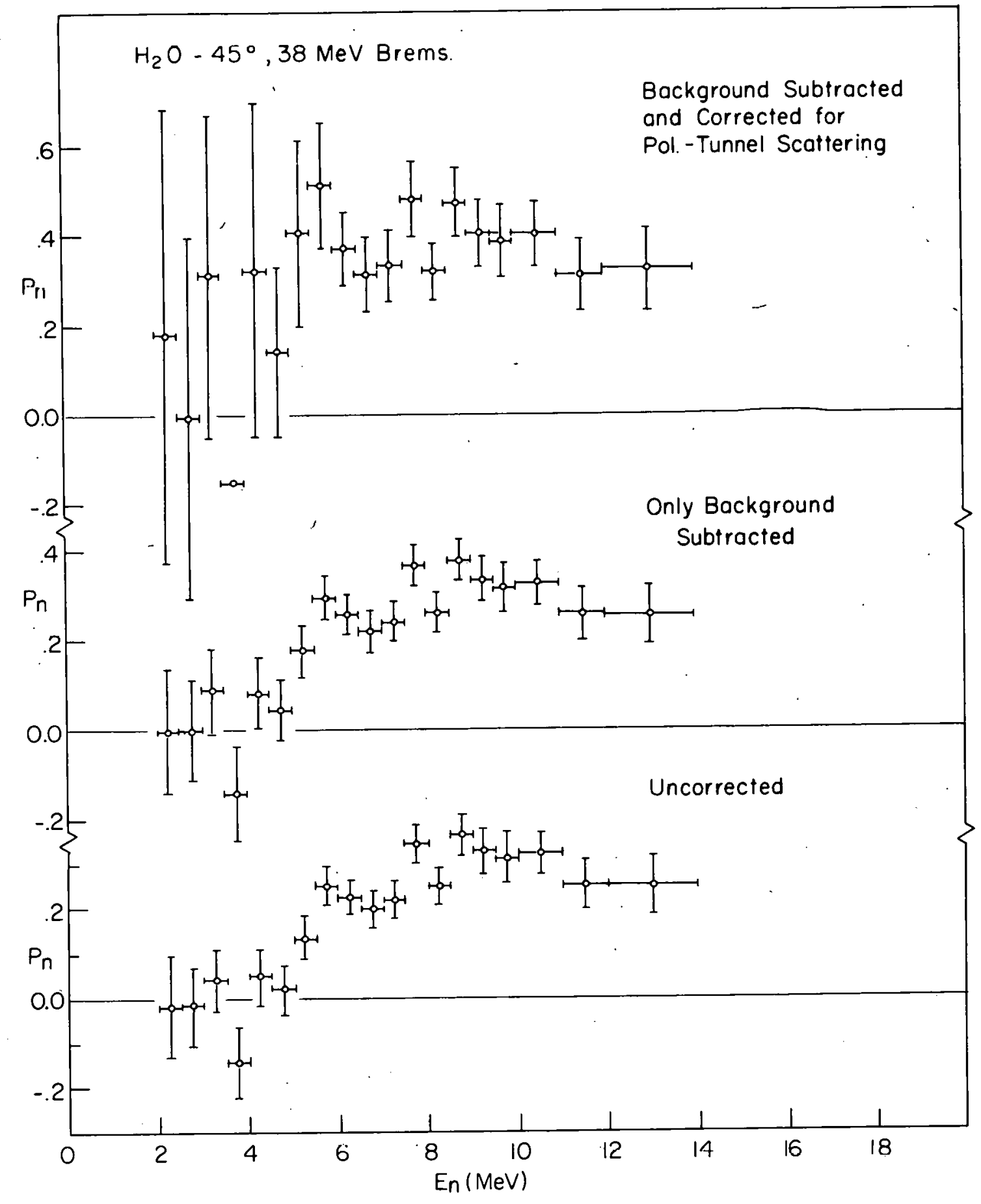

Fig. V-7 


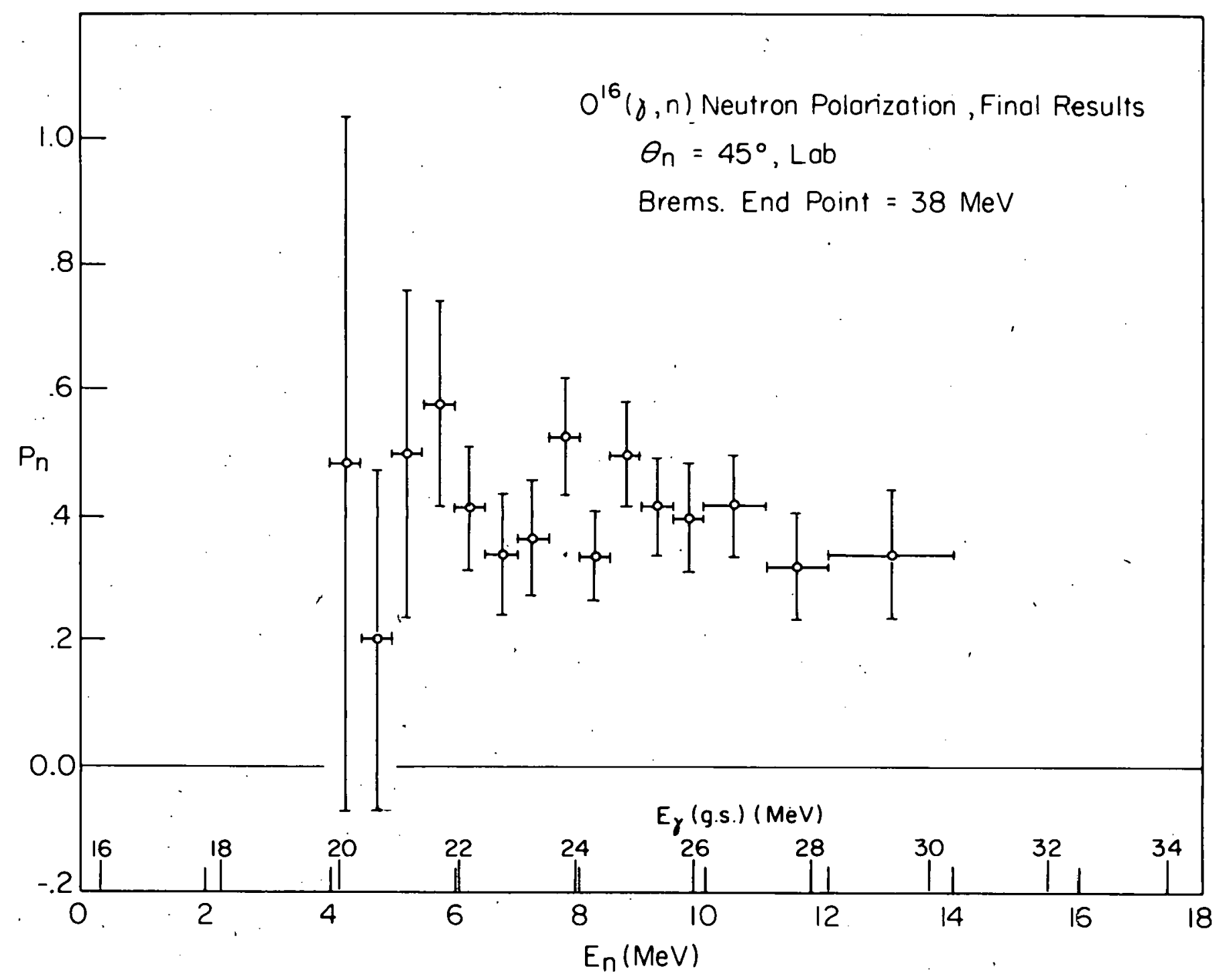

Fig. V-8 


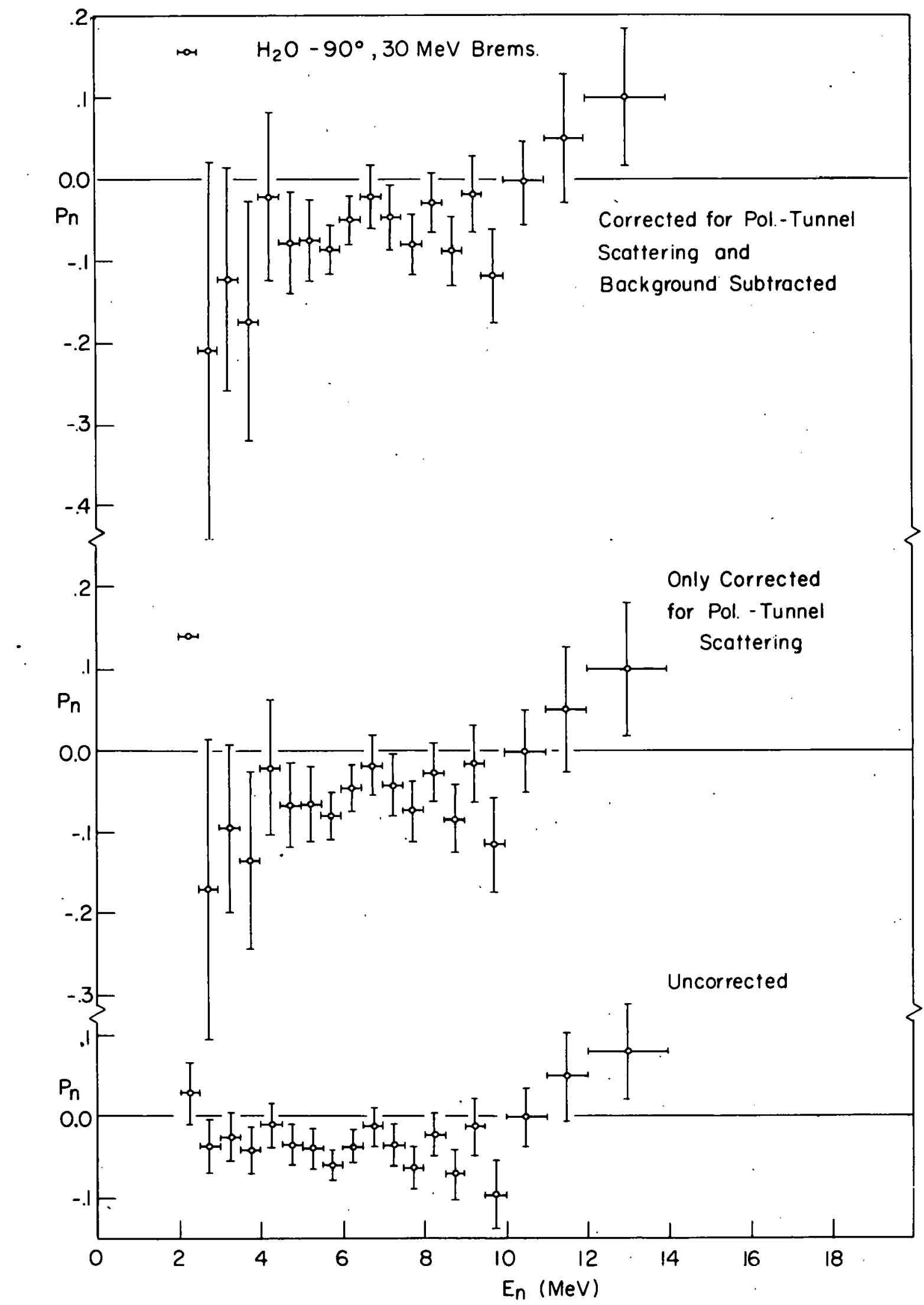

Fig. V-9 
$-129=$

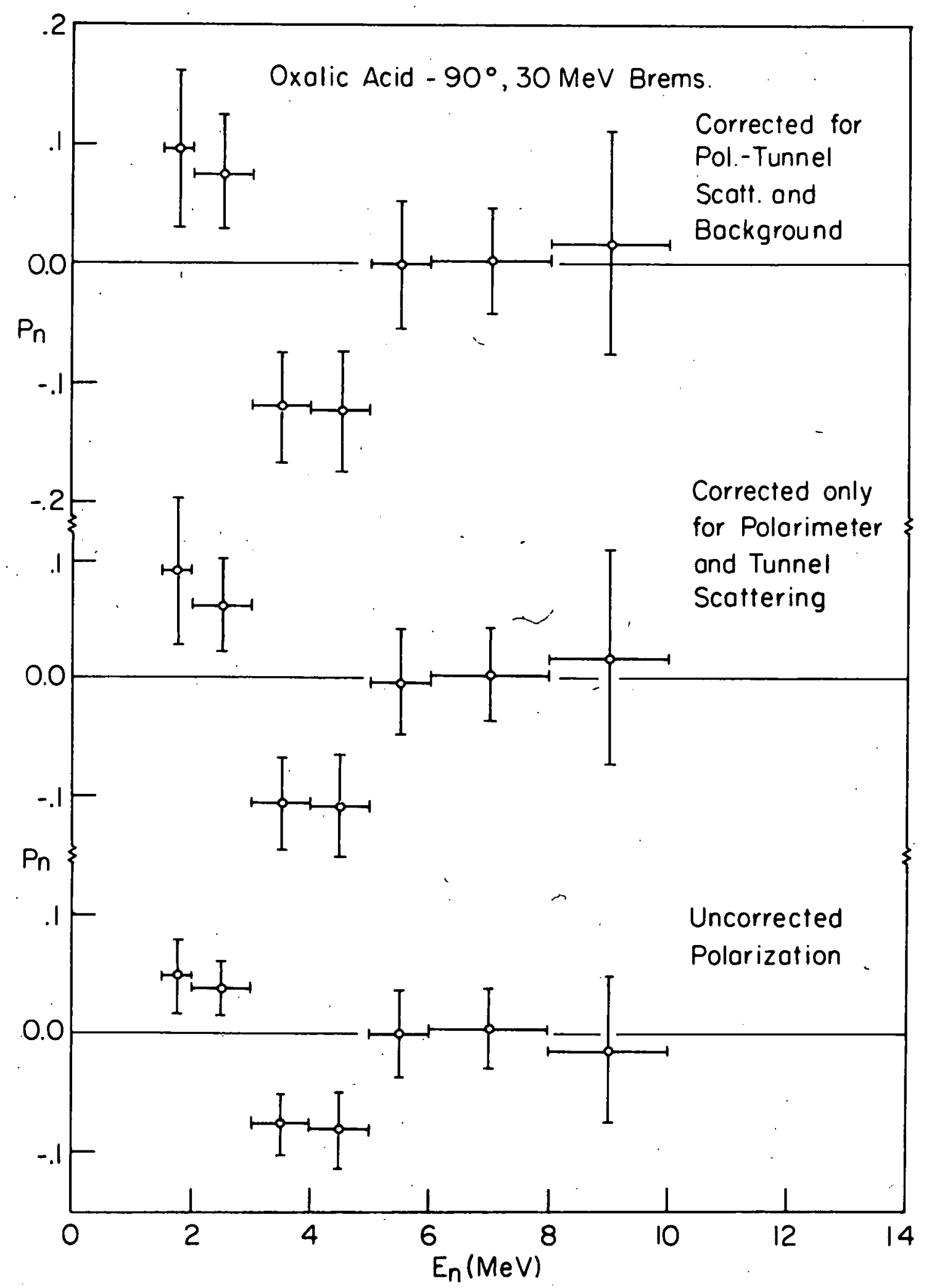

Fig. V-10 


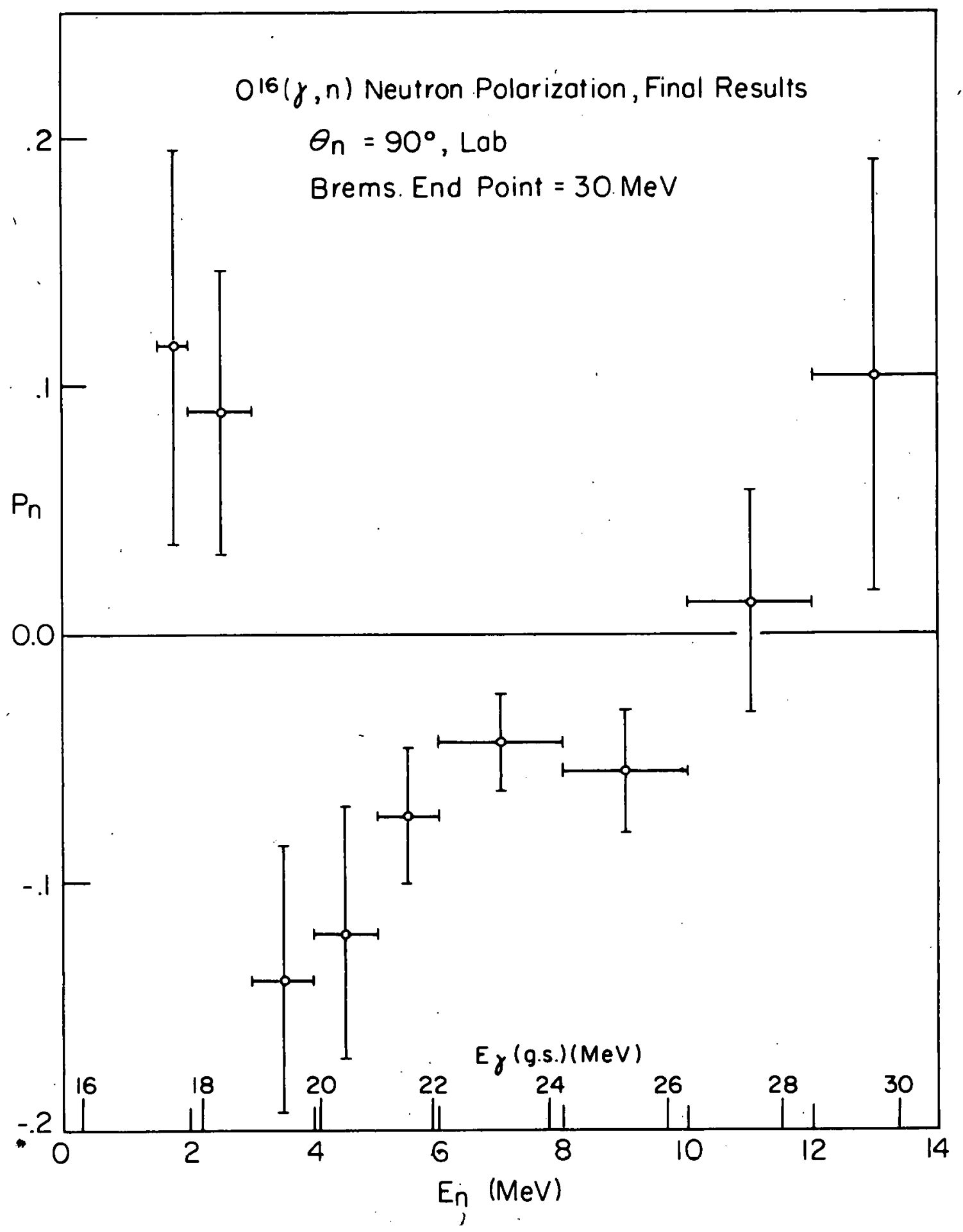

Fig. V-11 


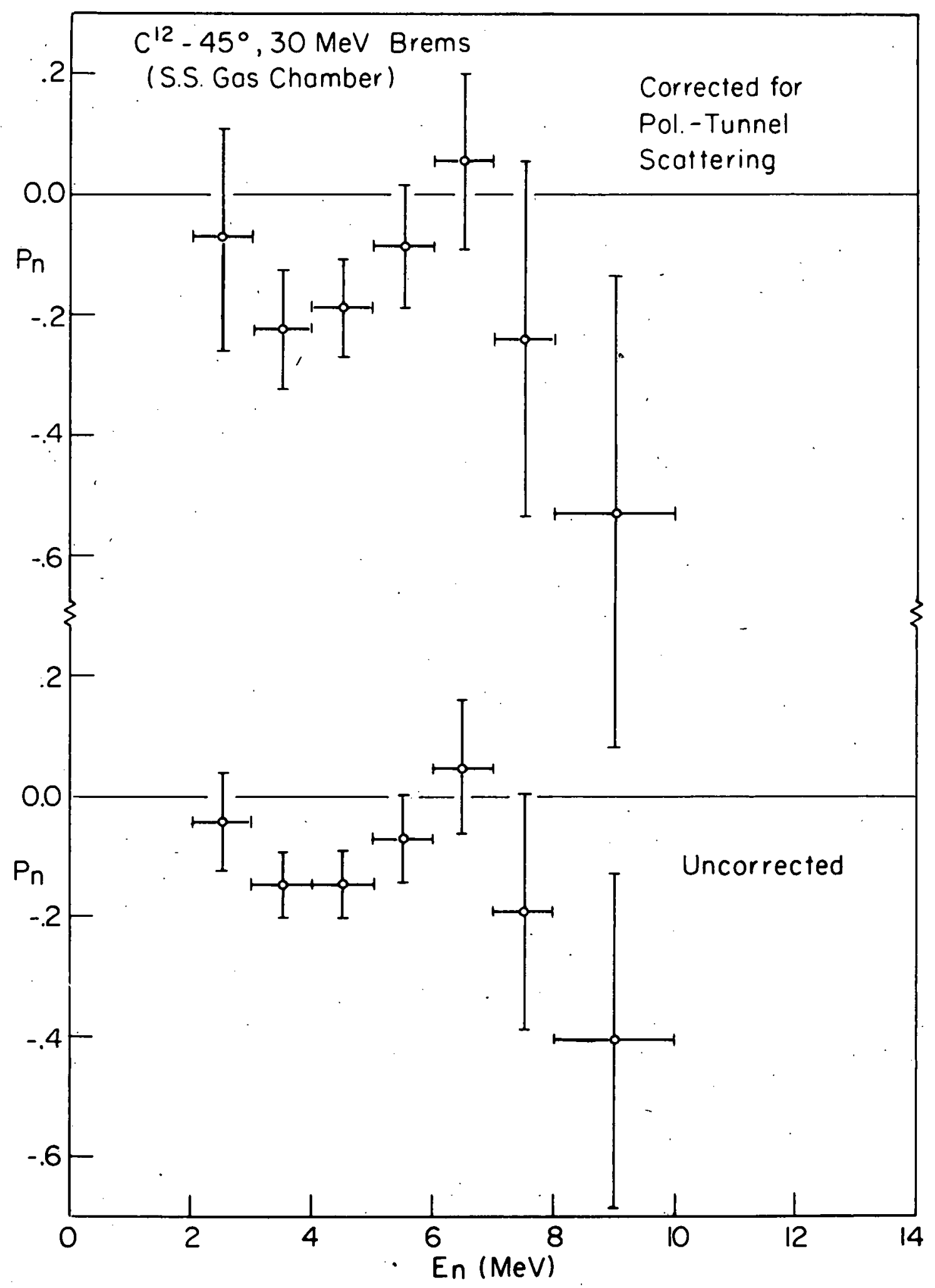

Fig. V-12 


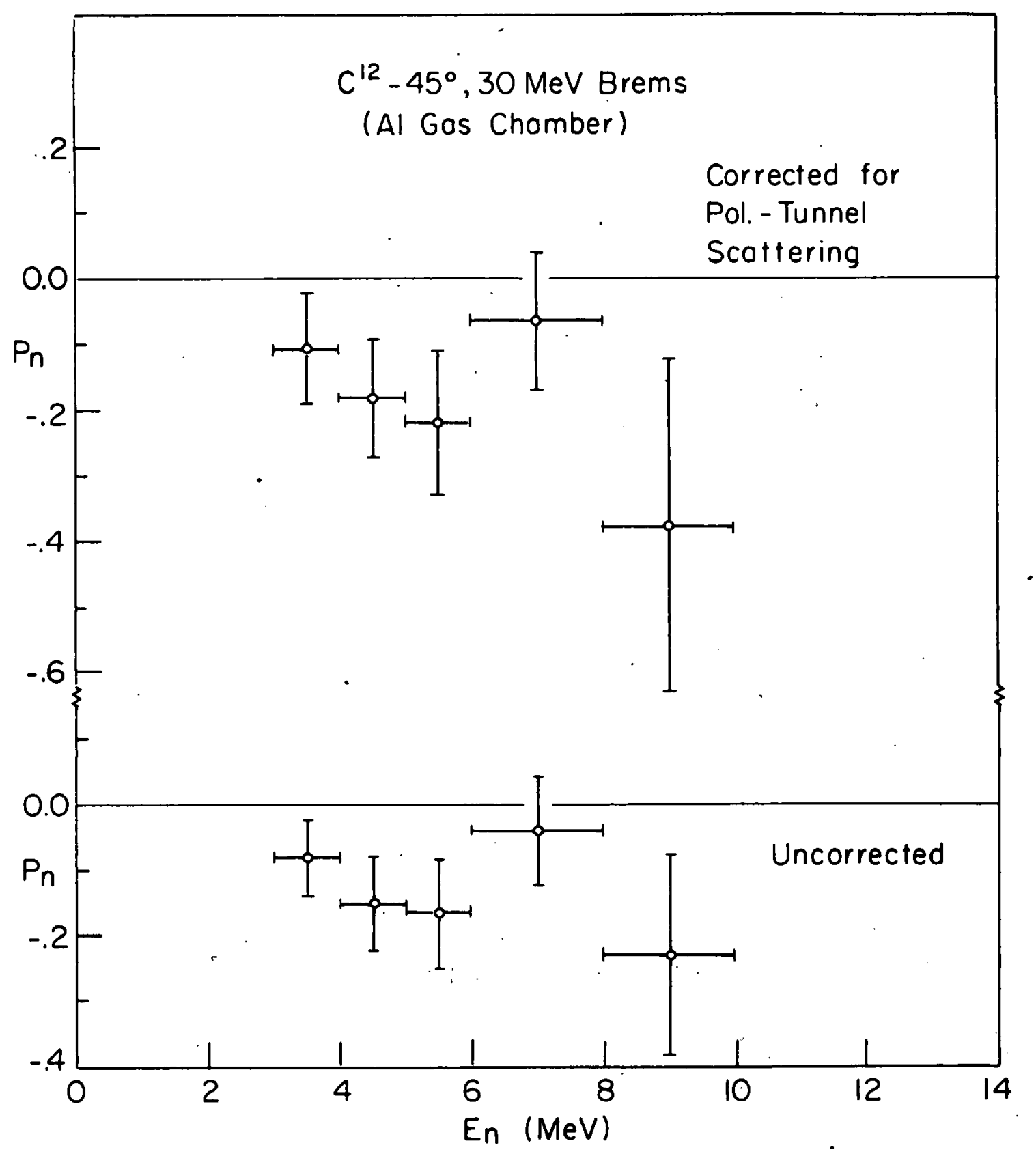

Fig. V-1.3 


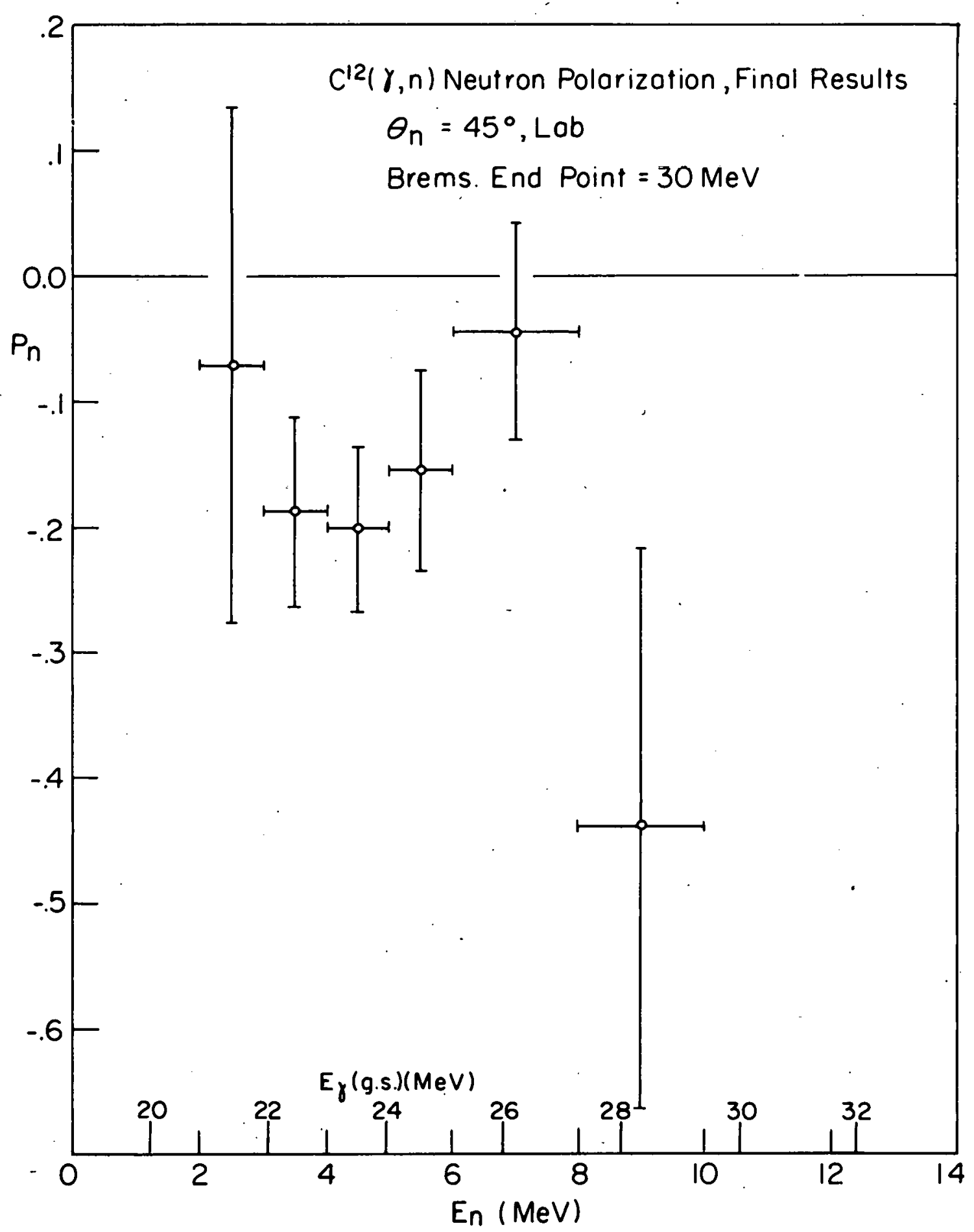

Fig. V-14 


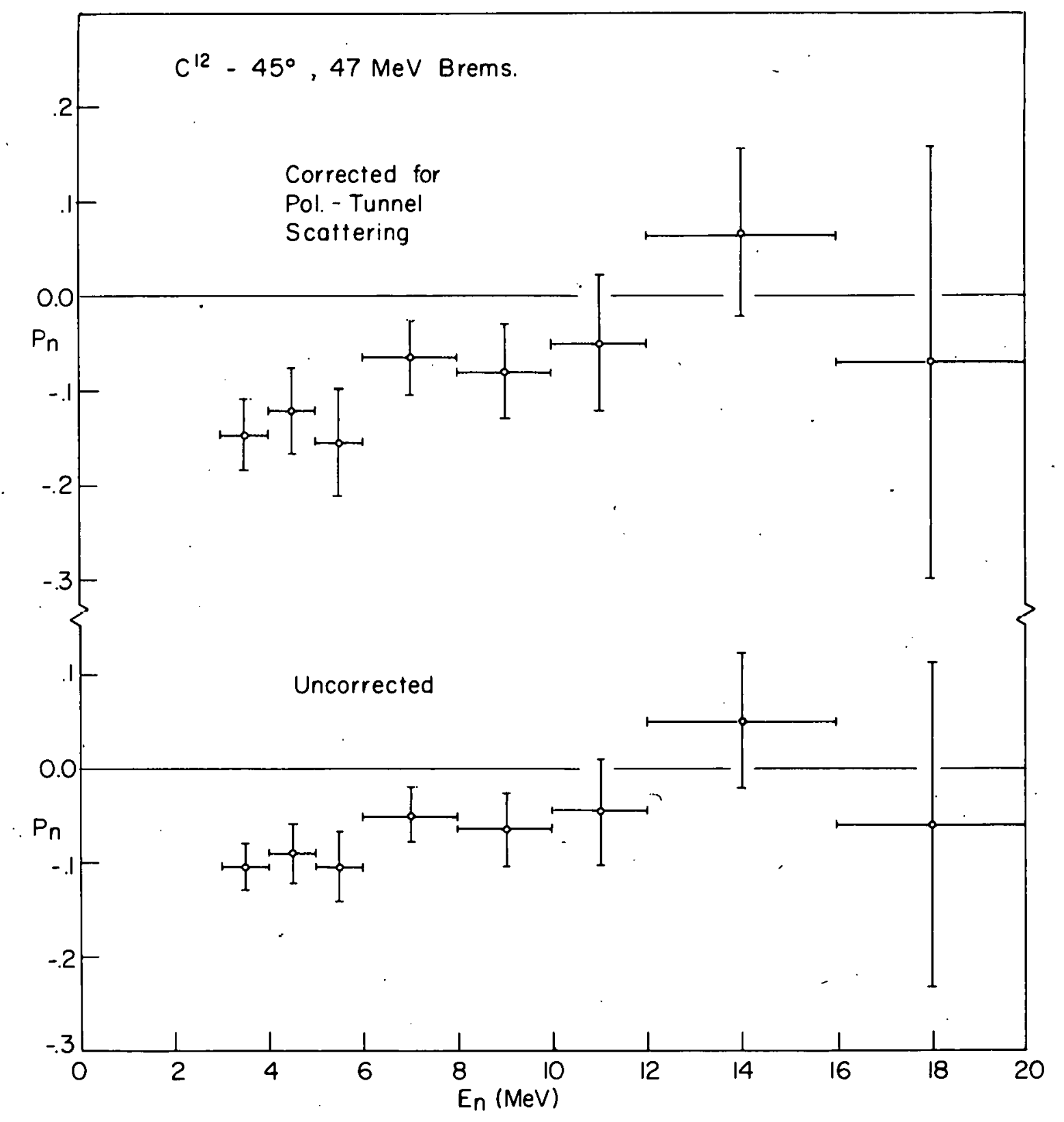

Fig. V-15 


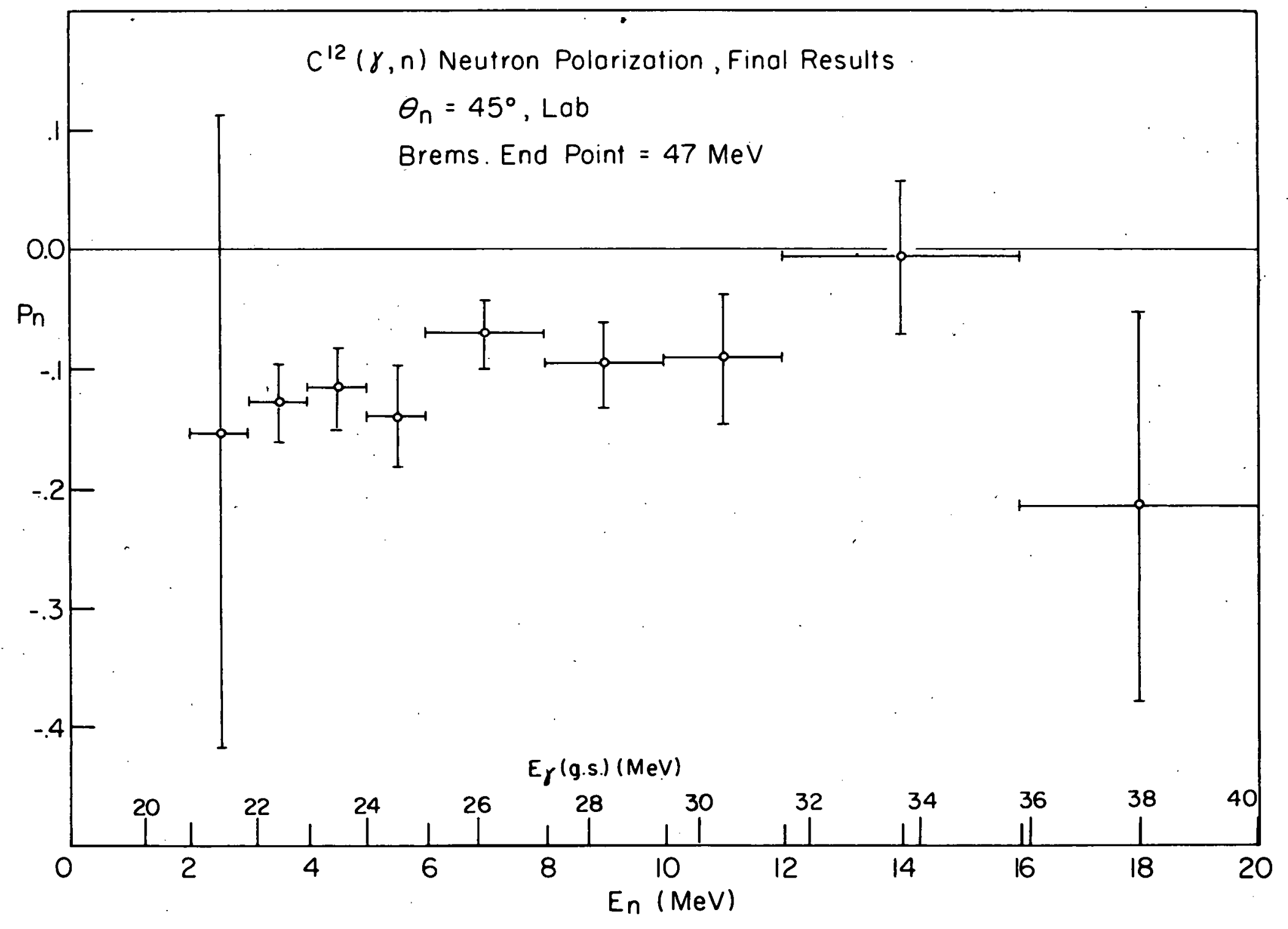

Fig. V-16 


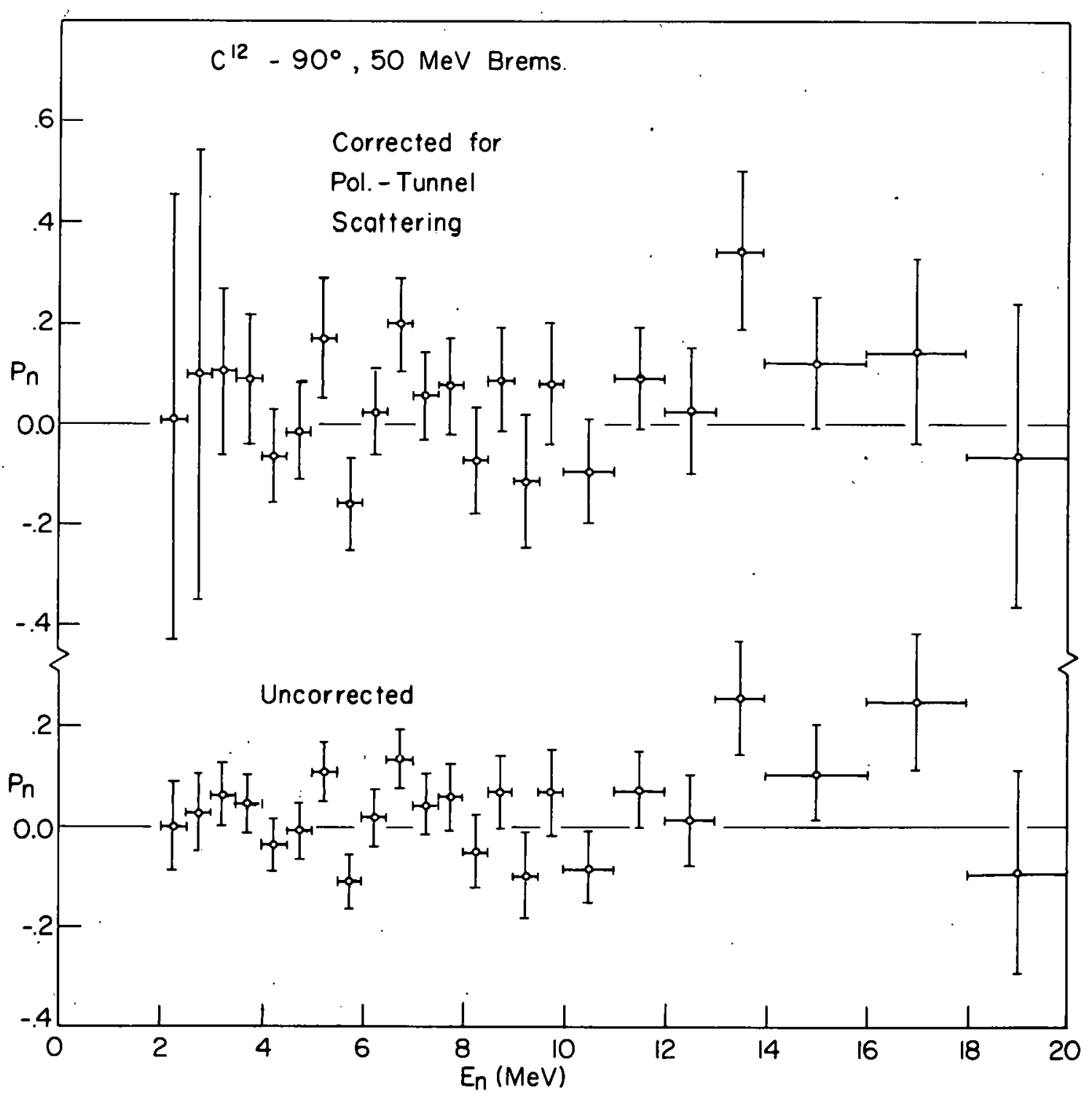

Fig. V-17 


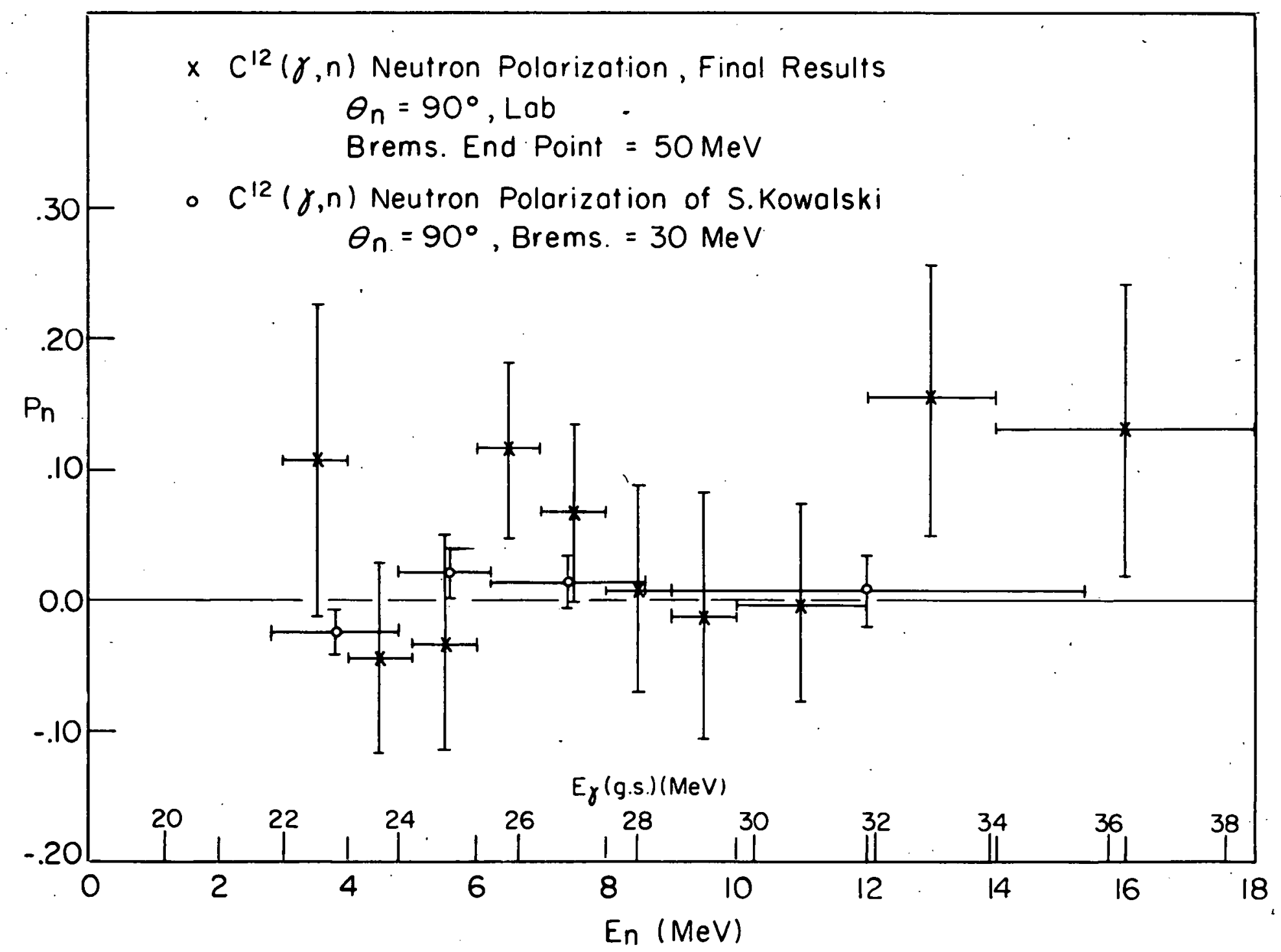

Fig. V-18 


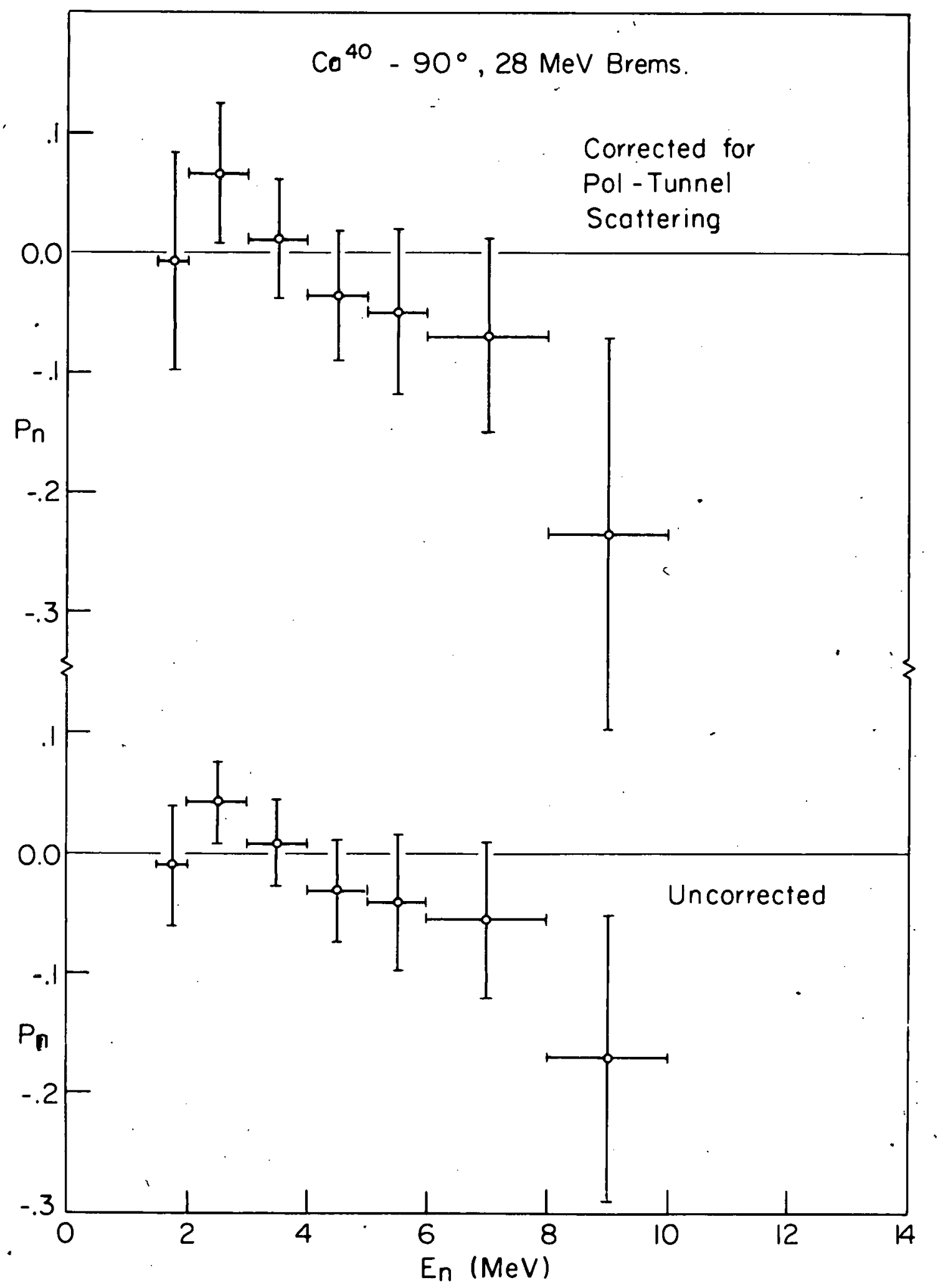

Fig. V-19 


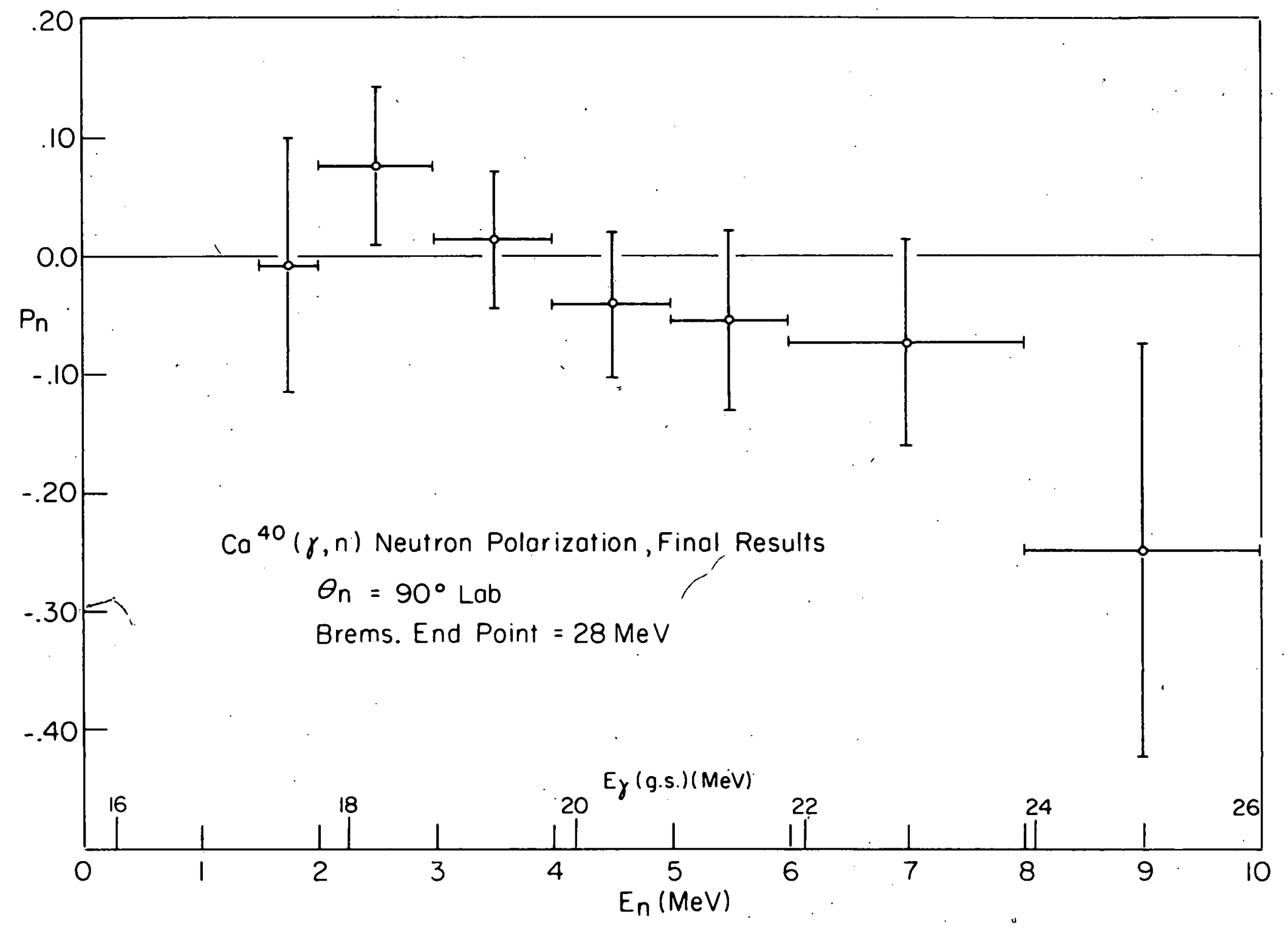




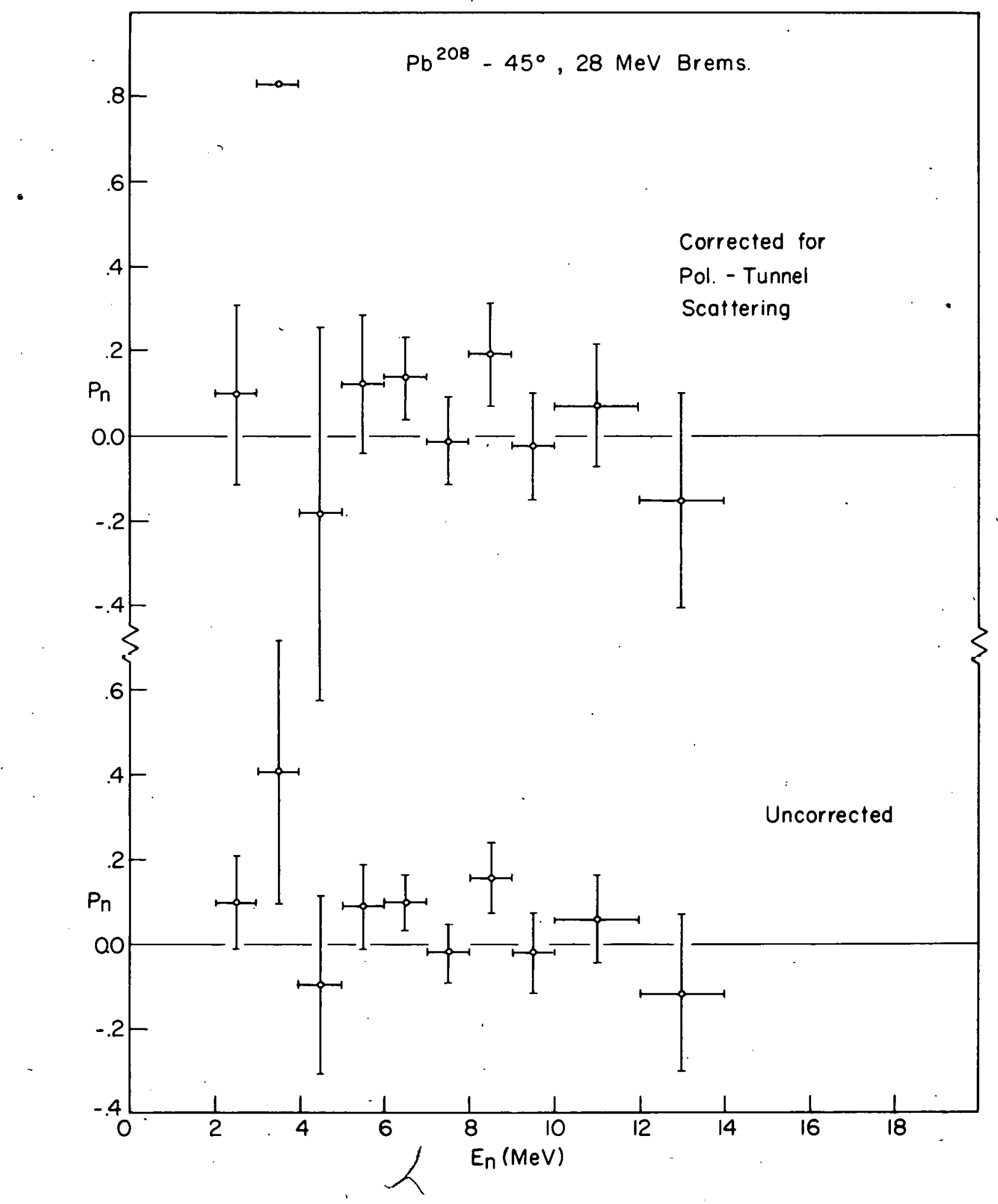

Fig. V-.21 


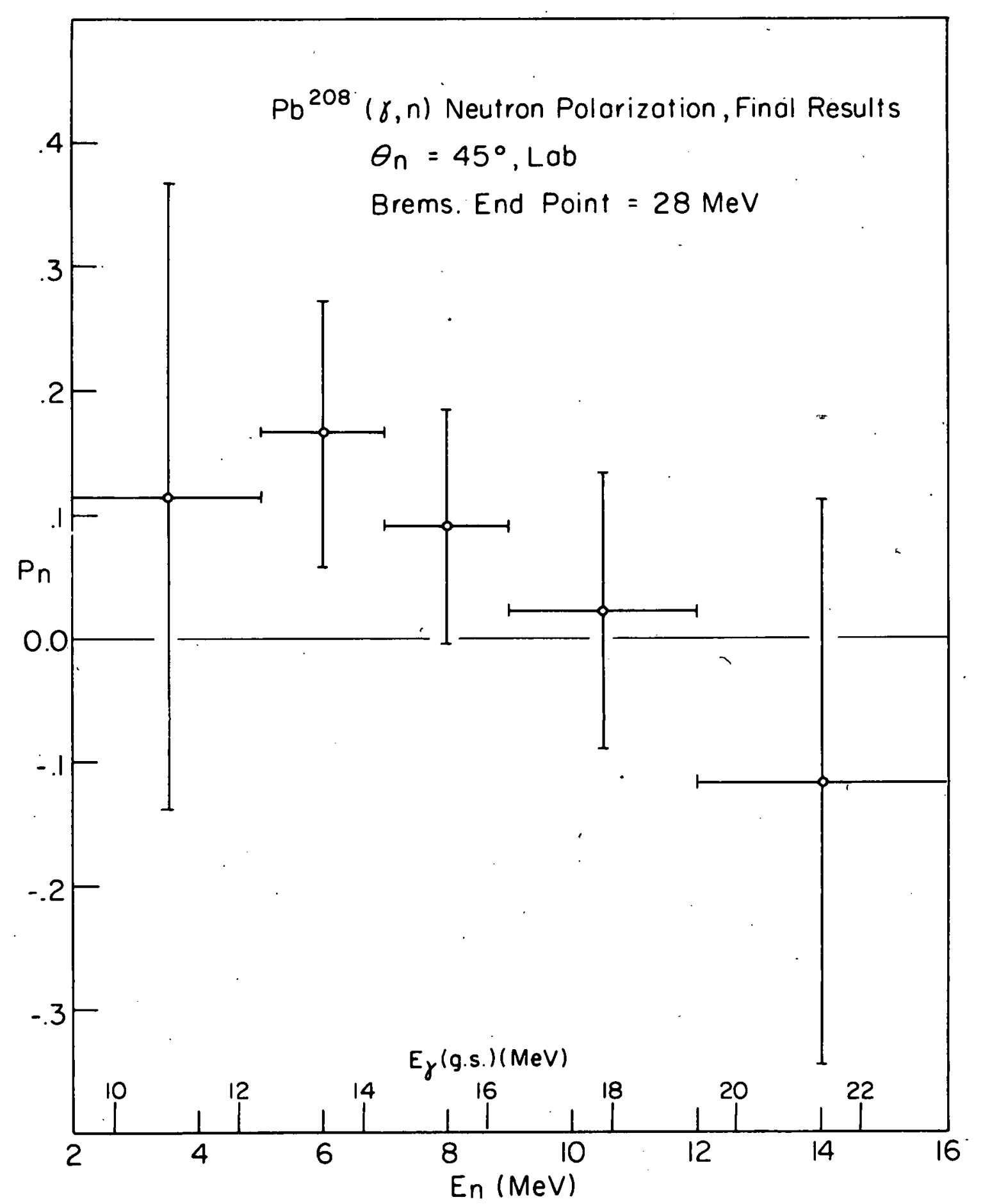

Fig. V-22 


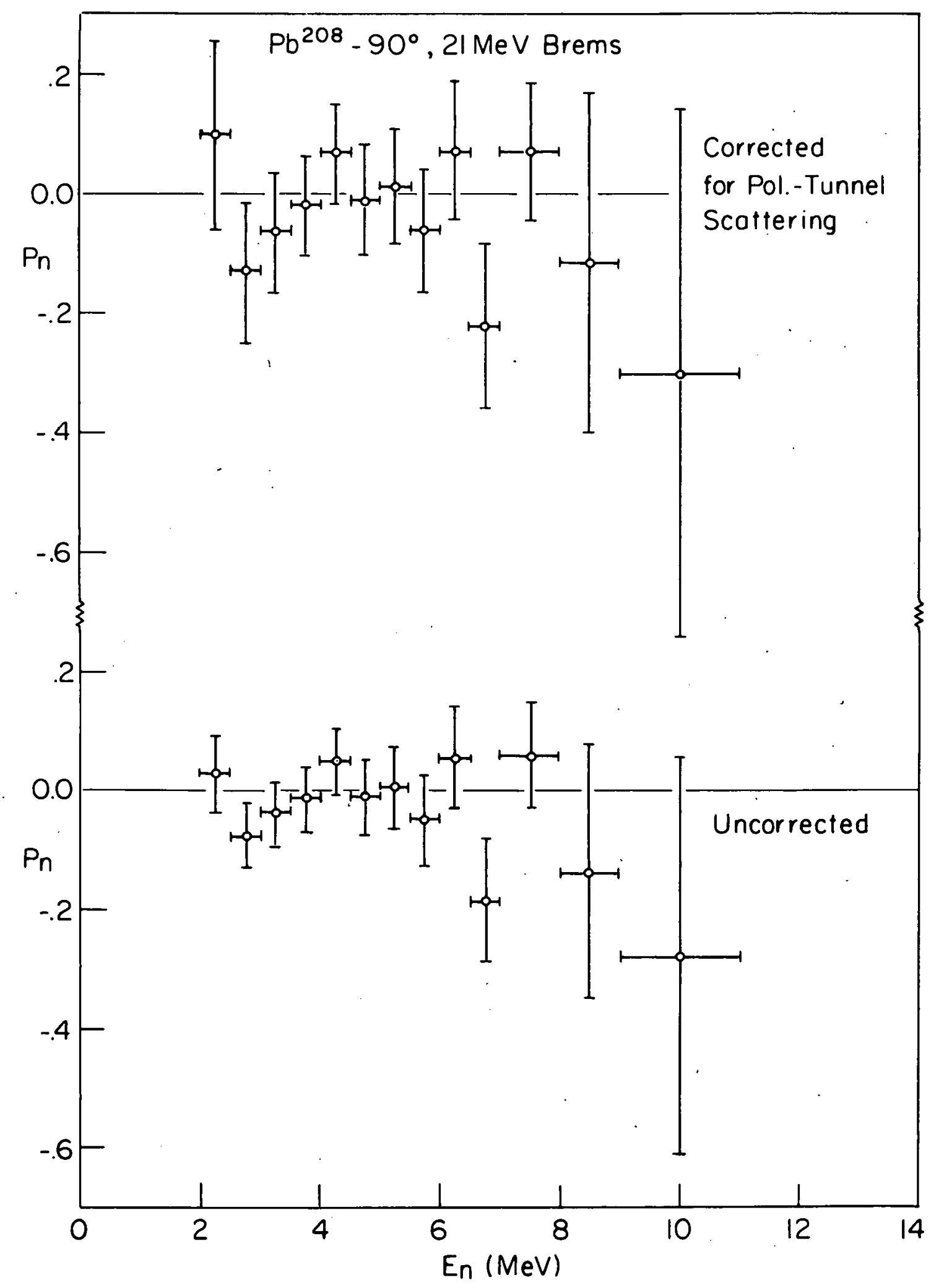

Fig. V-23 


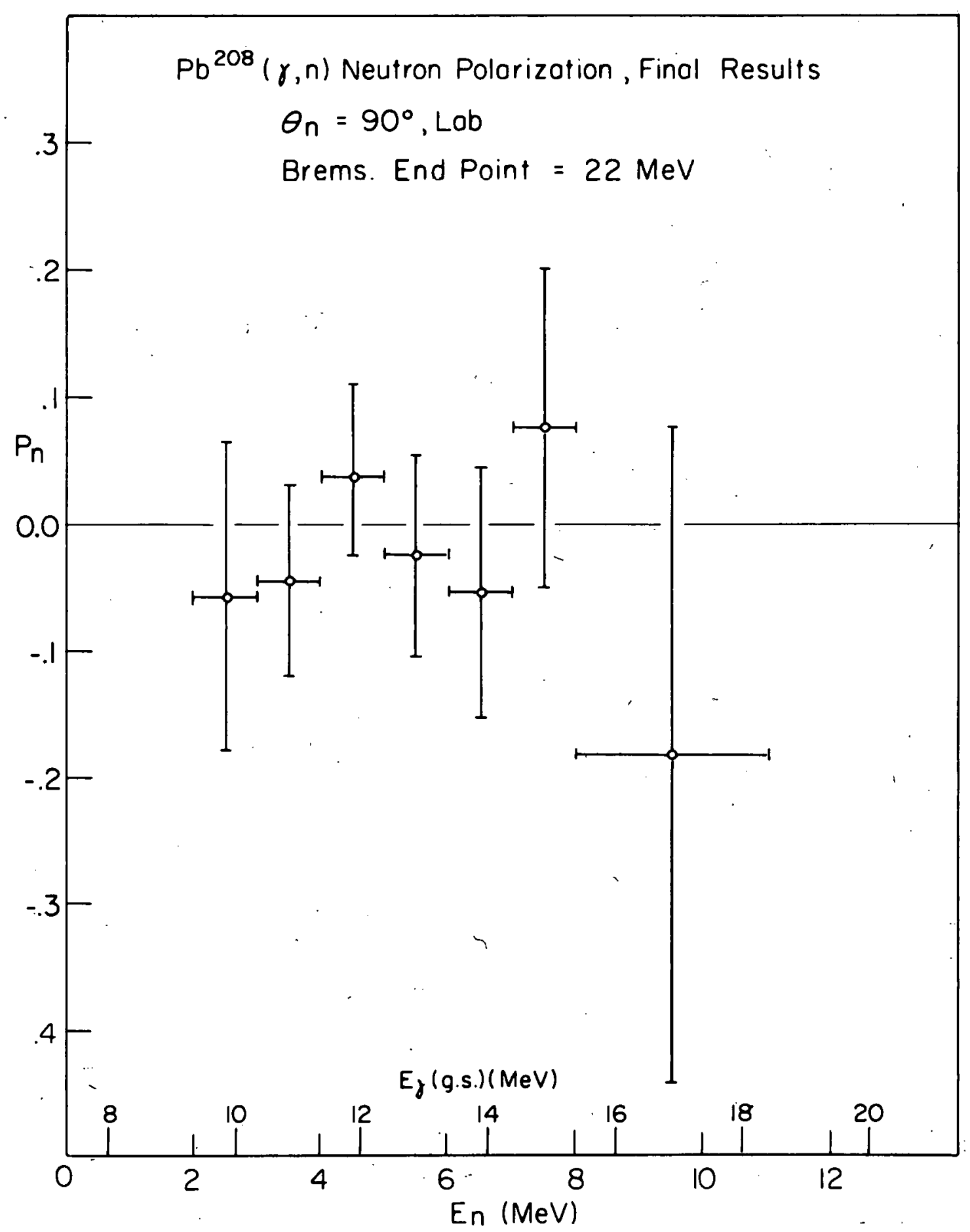

Fig. V-24 


\section{CHAPTER VI}

RESPONSE FUNCTION OF THE POLARIMETER; CORRECTIONS FOR SCATTERING BY THE POLARIMETER AND TUNNEL; CORRECTIONS FOR SCATTERING WITHIN THE PHOTONEUTRON TARGET.

A. Calculation of the Corrections for Scattering by the Polarimeter and Tunnel

1. General Description of the Scattering Corrections The neutron polarization measurements must be corrected for scattering from the polarimeter gas chamber and the tunnel walls, a general case of which iș illustrated in fig. VI-1. Many of the doubly scattered neutrons like that in fig. VI-1 will satisfy the timing requirements of the gas-counter and side-detector triggerings, and thus will be indistinguishable from neutrons scattering only from the He. The polarization measurements must be corrected for the presence of these dubly scattered neutrons.

The effect of the doubly scattered neutrons on the measured $\mathrm{He}^{4}$ recoil spectrum for a specified side-detector is illustrated in the two graphs of fig. VI-2. A monoenergetic neutron beam of $\mathrm{E}_{\text {in }}=14.7 \mathrm{MeV}$ is incident on the gas-counter and the plot shows the $\mathrm{He}^{4}$ recoil spectrum measured in coincidence with a side-detector at a central scattering angle $\theta_{0}=155^{\circ}$. The energy scale for the $\mathrm{He}^{4}$ recoils is labeled with the energy of an incident neutron which would give rise to that energy recoil by scattering at angle $\theta_{0}=155^{\circ}$. The first graph shows the expected recoil spectrum in the absence of any doubly scattered neutrons, with the width of the peak being determined by the gas-counter resolution for the $\mathrm{He}^{4}$ recoil energy and the spread 


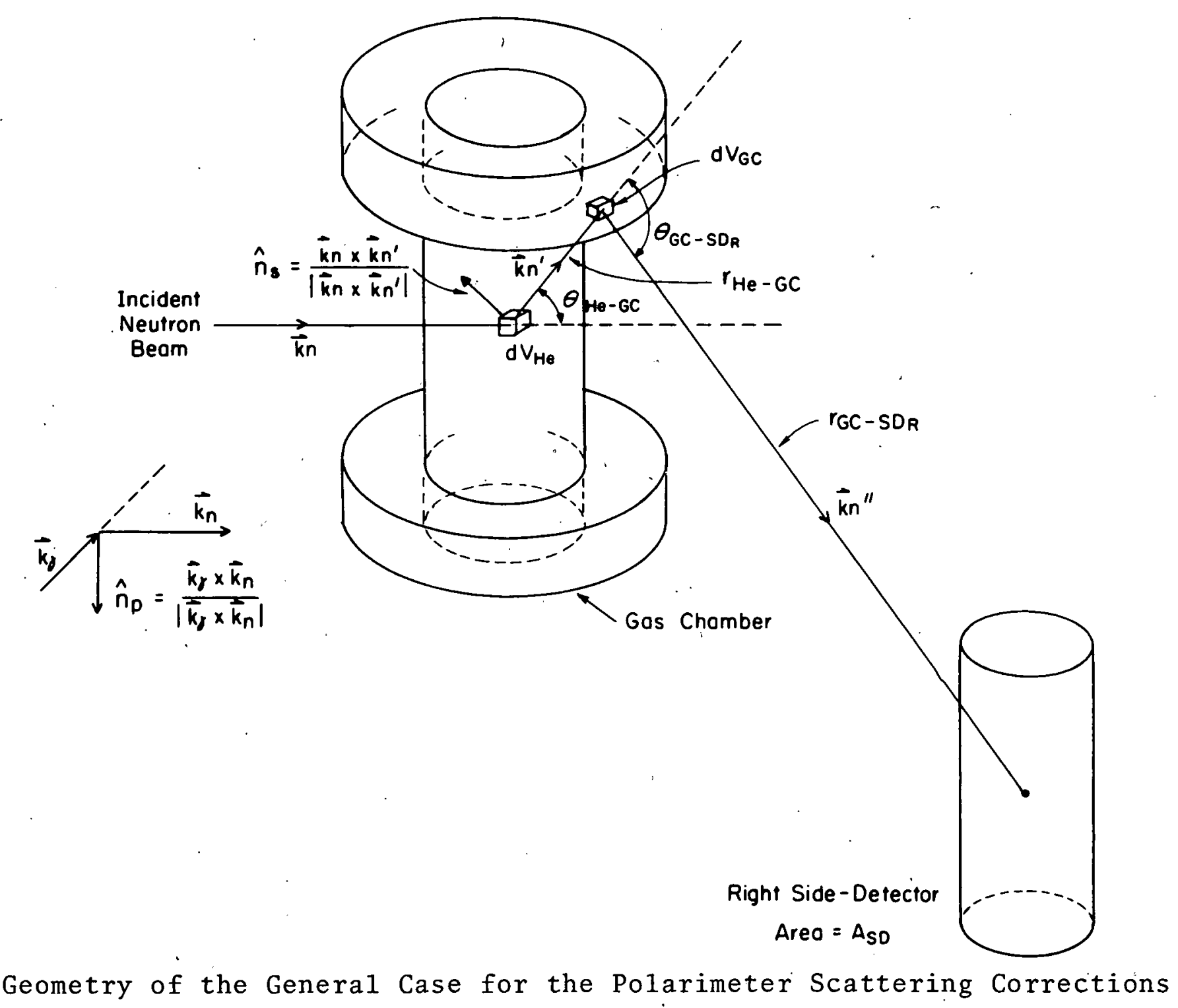

Fig. VI-1 


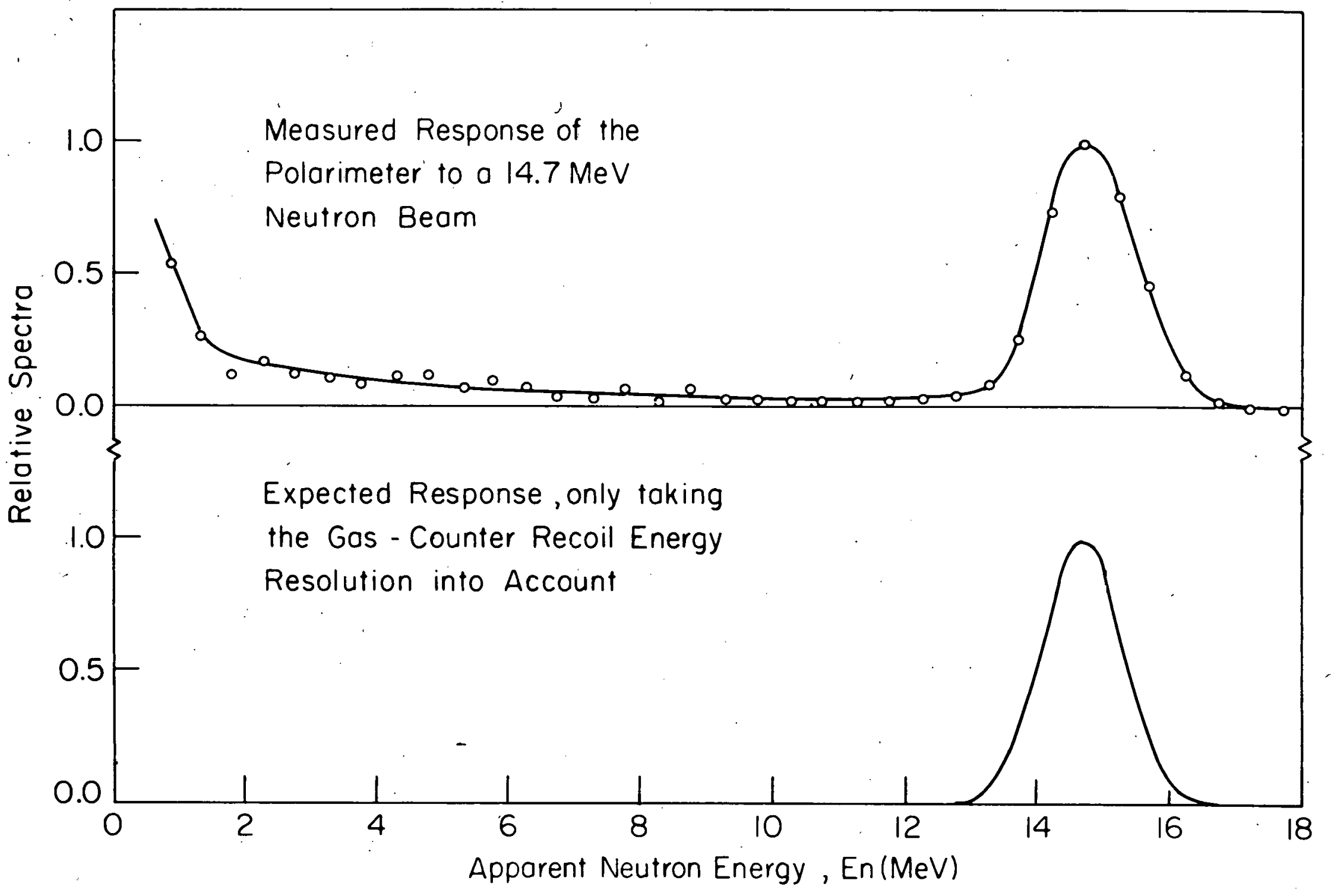

Measured and Ideal Response for $14.7 \mathrm{MeV}$ Neutrons

Fig. VI-2 
in scattering angles because of the non-zero dimensions of the gas-counter and the side-detector. The second graph shows an actual measured spectrum for $14.7 \mathrm{MeV}$ neutrons and a side-detector at $\theta_{0}=155$ degrees.

For purposes of calculation the corrections for doubly scattered neutrons are split into three types. Type I corrections, which are described in detail in section A-2, come from double neutron scatterings involving the cylindrical walls of the gas chamber. Since the gas chamber walls are illuminated by the incident neutron beam there are two cases for Type I corrections: case $A$, when the neutron scatters first from the He and then from the gas chamber wall; case. B, illustrated in fig. VI-3, when the neutron scatters first from the gas chamber wall and then from the He. Type II corrections, described in section A-3, come from scattering first from the He and then from the flanges of the gas chamber. Calculation of these corrections must take account of multiple neutron scatterings in the flanges, since the flange dimensions are generally greater than a neutron mean free path. Type III corrections, described in section A-4, arise from neutrons scattering first from the He and then from the walls of the tunnel in which the polarimeter was located.

It is now necessary to define the scattering corrections more exactly, and to relate the notation of this chapter with that in eqs. IV.24 to IV.27 of chapter IV. We consider a polarization measurement made with a side-detector pair located at a neutron scattering angle $\theta_{0}$ and a center to center distance from the gas chamber of $r_{0}$. The neutrons incident on the polarimeter in the course of an experiment are split into a set of energy intervals, 


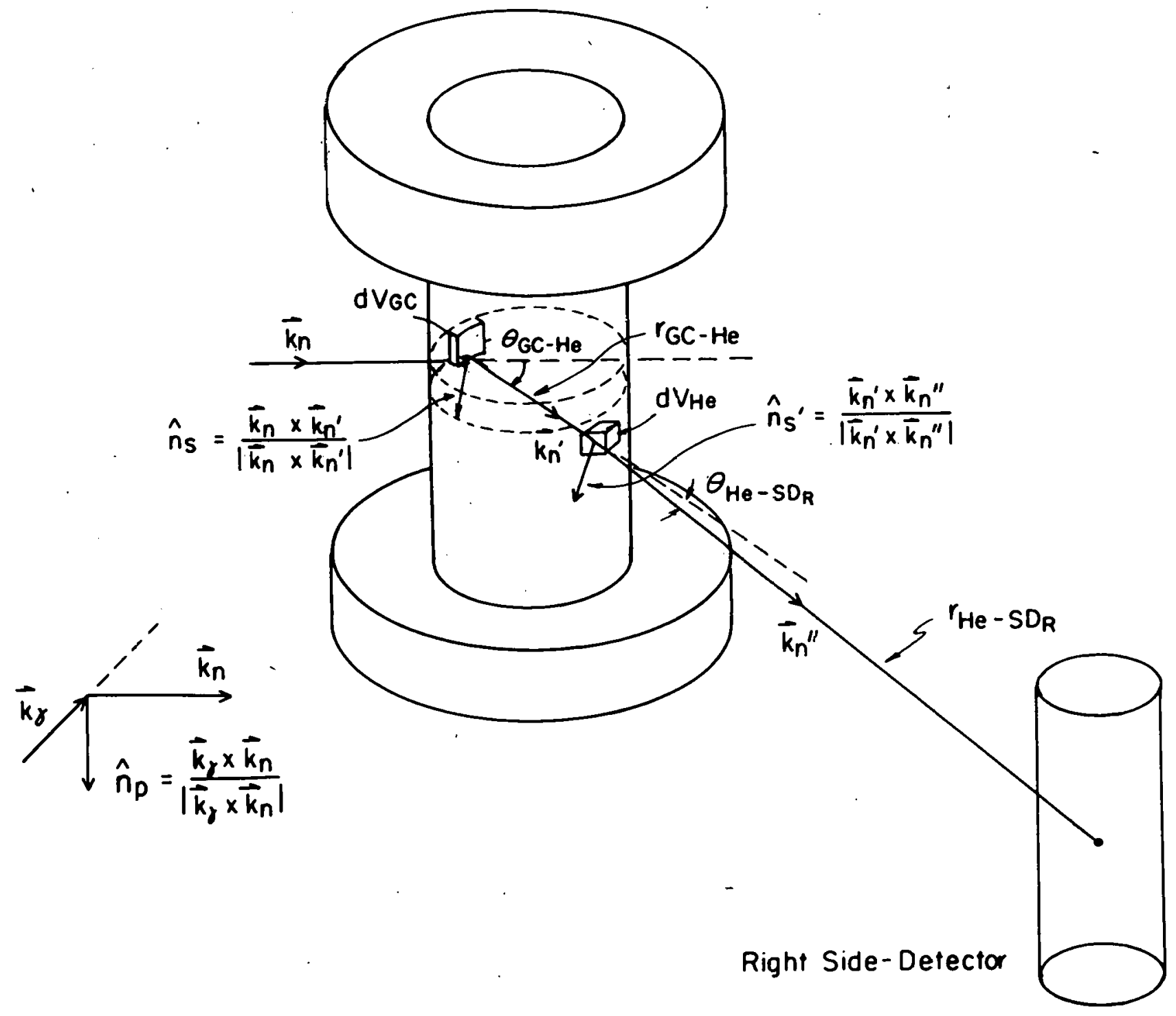

Geometry of the General Case when the Neutron Scatters first from the Gas Chamber Wall Fig. VI - 3 
the $i$ 'th interval having $\mathrm{N}_{\text {in }}$ (i) neutrons with an average energy of $\mathrm{E}_{\text {in }}(\mathrm{i})$, and polarization $\mathrm{P}_{\text {in }}(\mathrm{i})$. The measured neutron spectrum and polarization can be written as (eqs. IV.25 and IV.26)

$$
\begin{aligned}
& N_{m}(i)=\frac{N_{\text {in }}(i)}{1-k_{s c}(i)} \\
& P_{m}(i)=P_{s c}(i) k_{s c}(i)+P_{\text {in }}(i)\left(1-k_{s c}(i)\right)
\end{aligned}
$$

where

$$
\begin{aligned}
& k_{s c}(i)=\frac{N_{s C_{+}}(i)+N_{s C_{-}}(i)}{N_{\text {in }}(i)+N_{s C^{+}}(i)+N_{s C_{-}}(i)} \\
& P_{S_{C}}(i)=\frac{N_{S C}(i) P_{S C+}(i)+N_{S C_{-}}(i) P_{S C_{-}}(i)}{N_{S C^{+}}(i)+N_{S_{-}}(i)} \\
& N_{s C \pm}(i)=\sum_{j}^{\sum} N_{i n}(j) M_{S C \pm}(i, j)\left[\frac{1 \pm P_{j n}(j)}{2}\right] \\
& P_{S C \pm}(i)=\sum_{j} N_{i n}(j) M_{S C \pm}(i, j) P_{S C \pm}(i, j)\left[\frac{1 \pm P_{i n}(j)}{2}\right] / N_{S C \pm}(i)
\end{aligned}
$$

The matrices $M_{S C \pm}(i, j), P_{S C \pm}(i, j)$ can be calculated from equations of the form

$$
\begin{aligned}
N_{R \pm}(i, j)= & \Sigma^{\prime} n_{H e} d V_{H e} \frac{d \sigma}{d \Omega} H e\left(\theta_{H e-G C}\right)\left[1 \pm P_{H e}\left(\theta_{H e-G C}\right) \hat{n}_{\hat{p}} \cdot \hat{n}_{S}\right] \\
X & \frac{n_{G C} d V_{G C}}{r^{2} H e-G C} \frac{d \dot{\sigma}}{d \Omega} G C\left(\theta_{G C}-S D_{R}\right) \frac{A S D}{r^{2} G C-S D_{R}}{ }^{n} S D \\
& \delta\left(E_{a p}-E_{i n}(i)\right) \mid E_{i n}(j)
\end{aligned}
$$

VI. 3

where eq. VI.3 refers to fig. VI-1 and

$$
\begin{aligned}
\mid E_{i n}(j)- & \text { signifies that the calculation is carried out } \\
& \text { for incident neutrons of energy } E_{i n}(j) \text {, with }
\end{aligned}
$$


unit integ $\underset{\vec{k}}{a} \times \vec{k}$ \pm 1 along $\frac{\vec{k}_{\gamma} \times \vec{k}_{n}}{\mid \frac{k}{k} \times \vec{k}_{n}} \mid$.

$\delta\left(E_{a p}-E_{i n}(i)\right)$ - signifies that the $\mathrm{He}^{4}$ recoil energy is such that the apparent neutron energy $E_{\text {ap }}$, lies in the interval $E_{\text {in }}(i)$.

$\mathrm{N}_{\mathrm{R}_{ \pm}}(i, j) \quad-$ signifies that this is a scattering detected by the right side=detector, with an apparent neutron energy of $\mathrm{E}_{\text {in }}(\mathrm{i})$, and incident neutron energy $E_{i n}(j)$ with polarization \pm 1 .

$\mathrm{n}_{\mathrm{He}} \quad=$ number of $\mathrm{He}^{4}$ nuclei/cm ${ }^{3}$ in the gas volume.
$\mathrm{dV}_{\mathrm{He}} \quad=$ volume of a small He element.
$\mathrm{n}_{\mathrm{GC}} \quad=$ number of scattering nuclei/ $/ \mathrm{cm}^{3}$ in the gas chamber wall and flanges, or the tunnel wall.
$\mathrm{A}_{\mathrm{SD}}$
= effective area of the side-detectors.
$\mathrm{dV}_{\mathrm{GC}}$ = volume of a small element of the gas chamber wall and flanges, or the tunnel wall.

$\frac{\mathrm{d} \sigma}{\mathrm{d} \Omega} \mathrm{He}\left(\theta_{\mathrm{He}-\mathrm{GC}}\right), \mathrm{P}_{\mathrm{He}}\left({ }^{(\theta} \mathrm{He}-\mathrm{GC}\right)=1$ aboratory differential cross section and polarizing power for $\mathrm{He}^{4}(\mathrm{n}, \mathrm{n})$ scattering of neutrons of incident energy $E_{i n}(j)$ and at an angle $\theta_{\mathrm{He}-\mathrm{GC}}$.

${ }^{\theta} \mathrm{He}-\mathrm{GC} \quad=$ laboratory scattering angle for an incident neutron, from $\mathrm{dV}_{\mathrm{He}}$ to $\mathrm{dV}_{\mathrm{GC}}$. 
$\hat{n}_{p} \cdot \hat{n}_{s}=\frac{\left(\vec{k}_{\gamma} \times \vec{k}_{n}\right)}{\left|\vec{k}_{\gamma} \times \vec{k}_{n}\right|} \cdot \frac{\left(\vec{k}_{n} \times \vec{k}_{n}^{\prime}\right)}{\left|\vec{k}_{n} \times \vec{k}_{n}^{\prime}\right|}$, where $\vec{k}_{\gamma}$ is the photon wave vector, $\vec{k}_{n}$ the photo-produced neutron wave vector, and $k_{n}$, the $\mathrm{He}^{4}$ scattered neutron wave vector going from $\mathrm{dV}_{\mathrm{He}}$ to $\mathrm{dV}_{\mathrm{GC}} \cdot$

$\frac{\mathrm{d}}{\mathrm{d} \Omega} \mathrm{GC}\left({ }_{\mathrm{G}} \mathrm{GC}-\mathrm{SD}_{\mathrm{R}}\right)=$ laboratory differential czoss section for the gas chamber or tunnel material for a neutron of energy $E_{n}^{\prime}$ scattering at angle ${ }^{\theta} \mathrm{GC}_{-} \mathrm{SD}_{\mathrm{R}} ; \mathrm{E}_{\mathrm{n}}{ }^{\prime}$ is the neutron energy after scattering from $\mathrm{He}^{4}$.

${ }^{\theta} \mathrm{GC}-\mathrm{SD}_{\mathrm{R}}$

$=$ laboratory scattering angle for $\overrightarrow{\mathrm{k}}_{\mathrm{n}}{ }^{\prime} \rightarrow \overrightarrow{\mathrm{k}}_{\mathrm{n}}{ }^{\prime \prime}$, from $a V_{G C}$ to the right side-detector.

$\mathrm{r}_{\mathrm{He}-\mathrm{GC}}=$ distance between $\mathrm{dV}_{\mathrm{He}}$ and $\mathrm{dV}_{\mathrm{GC}}$.

${ }_{\mathrm{GC}-S D_{\mathrm{R}}}=$ distance between $d V_{G C}$ and the right side-detector.

$n_{S D} \quad=\quad$ detection efficiency per unit area of the side detector for a neutron of energy , $\mathrm{E}_{\mathrm{n}}^{\prime \prime} ; \mathrm{E}_{\mathrm{n}}^{\prime \prime}$ is the energy of the neutron after the second scattering.

The summation in eq.VI.3 goes over all volume elements $\mathrm{dV}_{\mathrm{He}}$ and $\mathrm{dV}_{\mathrm{GC}}$, with the prime indicating that a scattering is counted only if the He scattering and the detection in the side-detector obey the proper time relationship as set by the coincident circuit timing. A similar equation gives $\mathrm{N}_{L \pm}(i, j)$.

From the matrices $\mathrm{N}_{\mathrm{R} \pm}(i, j)$ and $\mathrm{N}_{\mathrm{L} \pm}(i, j)$, the matrices $M_{\text {Sct }}(i, j)$ and $P_{s c \pm}(i, j)$ can be calculated as 


$$
\begin{aligned}
& M_{S C \pm}(i, j)=\frac{N_{R \pm}(i, j)+N_{L \pm}(i, j)}{\overline{\bar{S}}_{H e}\left(\theta_{0}, E_{i n}(i)\right)} \\
& P_{S C \pm}(i, j)=\frac{1}{\overline{\bar{P}}_{H e}\left(\theta_{0}, E_{i n}(i)\right)}\left[\frac{N_{R+}(i, j)-N_{L \pm}(i, j)}{\left.N_{R \pm}(i, j)+N_{L \pm}(i, j)\right]}\right.
\end{aligned}
$$

where $\overline{\overline{\mathrm{S}}}_{\mathrm{He}}\left(\theta_{0}, \mathrm{E}_{\text {in }}(\mathrm{i})\right)$ and $\overline{\mathrm{P}}_{\mathrm{He}}\left(\theta_{0}, \mathrm{E}_{\text {in }}(\mathrm{i})\right)$ are the averaged detection efficiency and polarizing power defined in eqs. IV.14 and IV.12, and calculated from quantities defined in Appendix II. The meaning of $M_{S C \pm}(i, j)$ and $P_{S C \pm}(i, j)$ is that if one neutron $/ \mathrm{cm}^{2}$ in the energy interval $E_{i n}(j)$ is incident on the gas chamber with polarization \pm 1 , then this produces, by double scattering, $M_{S C t}(i, j)$ neutrons of apparent energy in the interval $E_{\text {in }}(i)$, and with apparent poiarization $\mathrm{P}_{S C \pm}(i, j)$.

The next three sections, A-2, A-3, and A-4, describe how different contributions like eqs. VI.3 and VI.4 were calculated, but first some additional comments will be made about some of the quantities used in eq.VI.3.

The $\mathrm{He}^{4}(\mathrm{n}, \mathrm{n})$ cross section and polarizing power, $\frac{\mathrm{d} \sigma}{\mathrm{d} \Omega} \mathrm{He}\left(\theta_{\mathrm{He}-\mathrm{GC}}\right)$ and $\mathrm{P}_{\mathrm{He}}\left(\theta_{\mathrm{He}-\mathrm{GC}}\right)$, were.calculated from the phase shifts used by S. Kowalski(46), the DGS phase shifts ${ }^{(54)}$ with the d-wave phase shifts set to zero. The newer phase shifts of Hoop and Barschall(53) were received after the scattering correction matrices had been calculated, and since the differences in the $\mathrm{He}^{4}(\mathrm{n}, \mathrm{n})$ cross section and polarizing power were not significant in view of the other approximations made in calculating these matrices, the calculations were not redone.

The neutron scattering cross section of the gas chamber material, $\frac{d_{\sigma}}{d \Omega} G C\left({ }_{G C}{ }_{G}-S D_{R}\right)$, had one of three forms. First was 
the elastic neutron scattering cross section, which was obtained by interpolation from a table of experimental cross sections

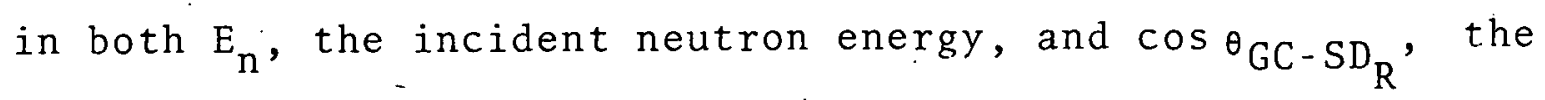
cosine of the neutron scattering angle. Since aluminum, the lightest nucleus comprising the gas chamber material, is 27 times as massive as the neutron, all elastic scattering was assumed not to alter the neutron's energy.

The second form of cross section was inelastic neutron scattering. The neutrons were taken to have an evaporation spectrum

$$
\frac{d N}{d E_{n}^{\prime \prime}}=C \underset{T^{Z}}{E_{n}^{\prime \prime}} \quad e^{-E_{n}{ }^{\prime \prime} / T}
$$

VI. 5

where

$$
T=\frac{1+\sqrt{1+4 \mathrm{a}\left(\mathrm{E}_{\mathrm{n}}{ }^{\prime \prime}-\mathrm{E}_{\mathrm{n}}{ }^{\prime \prime}\right)}}{2 \mathrm{a}}
$$

$$
\text { VI. } 6
$$

and

$\mathrm{C}=\mathrm{a}$ constant. related to the total inelastic cross, section.

$\mathrm{E}_{\mathrm{n}}{ }^{\prime}=$ energy of the incident neutron

$E_{n}^{\prime \prime}=$ energy of the outgoing neutron.

$a=a$ constant for a given nucleus; the value of a used for aluminum and iron (stainless steel) is $8 \mathrm{MeV}^{-1}$.

Eqs. VI.5 and VI.6 are based on the results of several papers on inelastic neutron scattering $(61-64)$, and àre a compromise on several possible forms. Here the total inelastic cross section was split up into six groups of different energy outgoing neutrons. The six inelastic groups were obtained from eq. VI.5 by neglecting the dependence of $T$ on $E_{n} "$. The outgoing 
spectrum was split into groups centered on $E_{n}^{\prime \prime}=.5 \mathrm{~T}, \mathrm{E}_{\mathrm{n}}^{\prime \prime}=1.0 \mathrm{~T}$, etc. If we set $E_{n}^{\prime \prime}=f T$ then eq. VI.6 can be solved to give

$$
E_{n}^{\prime \prime}=\frac{f}{2 a} \cdot\left[(1-f)+\sqrt{(1-f)^{2}+4 a E_{n}^{\prime}} \quad \text { VI. } 7\right.
$$

Eq. VI.7 gives $\mathrm{E}_{\mathrm{n}}$ ". the inelastic scattered neutron energy, for a given value of $f$. The fraction of the inelastic cross section appropriate for, neutrons of this energy is given in Table VI-1. The scattering is taken to be isotropic. Since little data on the polarization of elastic and inelastic neutron scattering exist, polarization in the second scattering was neglected. For incident neutron energies $E_{n}^{\prime}$ greater than the $(n, 2 n)$ threshold the inelastic cross section had the $(n, 2 n)$ cross section added to it to account for the second emitted neutron, and was atmost a $10 \%$ correction.

The third type of cross section for $\frac{d_{\sigma}}{d_{\Omega}} G C$ was inelastic scattering of the neutron with emission of a $\gamma$-ray and subsequent detection of the $r$-ray in tne siae-detector. lhe cross section for $\left(n, n^{\prime} \gamma\right)$ used is the total inelastic and absorption neutron cross section, and isotropic emission of the $\gamma$-rays is assumed. For this case the side-detector efficiency, ${ }_{S D}$, is replaced by a $\gamma$-ray detection efficiency. The average energy and multiplicity of the $r$-rays is a function of the incident neutron energy, $E_{n}{ }^{\prime}$, with the values used for aluminum and iron being given in table $\mathrm{VI}-2$

The side detector neutron detection efficiency, " ${ }_{S D}$, was calculated using the hydrogen scattering cross section for neutrons, the density of hydrogen in toluene, and an assumed proportional counter energy dependence of the form $\left(E_{n^{\prime \prime}}-E_{t h}\right) / E_{n^{\prime \prime}}$, 
SPECTRUM OF INELASTICALLY

SCAIITERED NEUTRONS

$$
E_{n}{ }^{\prime}=f T\left(E_{n}\right)
$$

\begin{tabular}{|c|c|}
\hline$f$ & $\begin{array}{l}\text { Fraction of } \\
\text { Inelastic o used }\end{array}$ \\
\hline & \\
0.5 & 0.173 \\
1.0 & 0.182 \\
1.5 & 0.167 \\
2.0 & 0.191 \\
3.0 & 0.151 \\
4.0 & 0.119 \\
& $\sum=0.983$ \\
\hline
\end{tabular}

TABLE VI - 2

$\gamma$-RAY ENERGIES AND MULTIPLICITIES.

USED FOR INELASTIC NEUTRON SCATTERING

ALUMINUM

\begin{tabular}{|c|c|c|}
\hline$E_{n}$ & $E_{\gamma}$ & $N_{\gamma}$ \\
\hline 1.0 & - & - \\
1.5 & 0.84 & 1.0 \\
2.0 & 1.0 & 1.0 \\
2.5 & 1.1 & 1.0 \\
3.0 & 1.3 & 1.1 \\
4.0 & 1.3 & 1.3 \\
5.0 & 1.7 & 1.3 \\
6.0 & 2.1 & 1.3 \\
7.0 & 2.5 & 1.3 \\
14.0 & 2.0 & 1.0 \\
24.0 & 2.5 & 2.0 \\
\hline
\end{tabular}

STAINLESS STEEL

\begin{tabular}{|l|l|l|}
\hline$E_{n}$ & $E_{\gamma}$ & $N_{\gamma}$ \\
\hline 1.0 & 0.85 & 1.0 \\
1.5 & 0.85 & 1.0 \\
2.0 & 0.85 & 1.0 \\
2.5 & 0.85 & 1.05 \\
3.0 & 0.90 & 1.10 \\
4.0 & 1.0 & 1.2 \\
5.0 & 1.1 & 1.4 \\
6.0 & 1.2 & 1.6 \\
7.0 & 1.3 & 2.0 \\
14.0 & 1.0 & 1.0 \\
24.0 & 3.0 & 5.0 \\
\hline
\end{tabular}


where $E_{t h}$ is the threshold energy. More accurate calculations for such counters have been made by Verbinski. et al. (55) accounting for multiple collisions and other processes. The rough values for ${ }^{n} D^{d o}$ not differ significantly from the more accurate results of Verbinski et. al. considering the other approximations being made for these corrections.

2. Calculation of the Corrections for Scattering from the Cylinder Wall.

These corrections arise from two cases. Case A comes from scattering first from the He and then from the gas chamber cylinder wall. These corrections were calculated with eq.VI.3, the sum over $\mathrm{dV}_{\mathrm{GC}}$ only going over the volume of the cylinder wall.

Case B corrections, illustrated in fig. VI-3, come from scattering. first from the cylinder wall and then from the He. They are calculated from

$$
\begin{aligned}
& N_{R^{ \pm}}(i, j)=\Sigma \cdot \frac{n_{G C} d V_{G C}}{r^{2} G C-H e} \frac{d \sigma}{d \Omega} G C\left(\theta_{G C-H e}\right) n_{H e} d V_{H e} \frac{d \sigma}{d \Omega} H e\left(\theta_{H e}-S D_{R}\right) \\
& \left.x\left[1 \pm P_{H e}\left(\theta_{H e}-S D_{R}\right)\left(\hat{n}_{p} \cdot \hat{n}_{s}\right)\left(\hat{n}_{s} \cdot \hat{n}_{s}^{\prime}\right)\right] \frac{A_{S D}{ }^{n} S D}{r_{H e}^{2}-S D_{R}} \delta\left(E_{a p}-E_{i n}(i)\right)\right|_{E_{i n}(j)}
\end{aligned}
$$

\section{VI . 8}

where most symbols have already been defined in eq.VI.3, and the new ones are as shown in fig. VI-3. A similar equation gives $\mathrm{N}_{L \pm}(i, j)$.

The results from calculations for Case A and Case $B$ corrections give a component, $M_{c y \pm}(i, j)$ and $P_{c y \pm}(i, j)$, of the matrices $\mathrm{M}_{\mathrm{SC} \pm}(i, j)$ and $\mathrm{P}_{\mathrm{SC} \pm}(i, j)$, which later have the flange and tunnel corrections added to form the complete scattering correction matrices. 
3. Calculation of the Corrections for Scattering from the Flanges - Multiple Scattering Effects.

For neutrons in the energy region 1 to $20 \mathrm{MeV}$ all

flange dimensions are greater than a neutron mean free path, so two new aspects come into the calculations of the scattering corrections. First, the doubly scattered neutrons must be attenuated as they enter and leave the flanges, and second, multiple scattering of the neutrons becomes significant and the contribution from double and triple scattering in the flanges may exceed that from single scatterings. For the purpose of simplifying the calculations, the glass plates in the flanges were replaced by an equi-nuclear amount of material identical to the gas chamber i.e. aluminum or stainless steel. This is expected to be a good approximation, since the neutron cross sections are about the same, and the glass plates do not contribute greatly to the scattering corrections because any neutrons scatter'd by them must be attenuated by the material in the flanges before being able to strike a side-detector.

The corrections for flange scattering are calculated from eq. VI.3, with modifications for neutron attenuation on entering and leaving the flange. This gives (see fig. VI-1 for the geometry)

$$
\begin{gathered}
N_{R \pm}(i, j)=\Sigma^{\prime}\left(\text { terms in eq.VI.3) } e^{-d_{i n / \lambda i n} e^{-d} \text { out } / \lambda \text { out }}\right. \\
\text { VI. } 9
\end{gathered}
$$

where

$e^{-d_{i n} / \lambda i n}=$ transmission factor for the neutrons entering the flange. $d_{i n}$ is the distance through flange material which the incoming neutron travels. $\lambda_{\text {in }}$ is the mean free path for an incoming neutron 


$$
\text { of energy } E_{n}^{\prime} \text {. }
$$

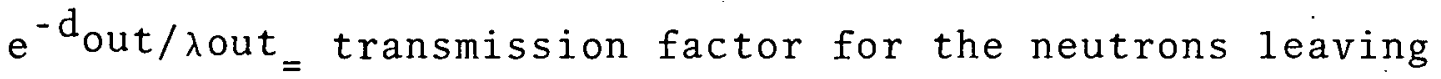
the flange. $d_{\text {out }}$ is the distance through flange material which the outgoing neutron travels. $\lambda$ out is the neutron (or $\gamma$-ray, when the third type of $\frac{\mathrm{d} \sigma}{\mathrm{d} \Omega} \mathrm{GC}$ is used) mean free path for an outgoing neutron of energy $E_{n}^{\prime \prime}\left(\gamma\right.$-ray of energy $\left.E_{\gamma}\left(E_{n}^{\prime}\right)\right)$. The neutron mean free paths, $\lambda_{\text {in }}$ and $\lambda_{\text {out }}$, are calculated using only the inelastic cross sections. Since elastic scattering from aluminum or iron leaves the neutron with most of its energy, and the elastic cross section for neutrons greater than a few MeV tends to be mostly in the forward direction, this should be a somewhat better approximation to the actual attenuation of the neutrons than using the total cross section. An equation similar to VI.9 gives $\mathrm{N}_{\mathrm{Lt}}(i, j)$ for the flange scattering. The results of these calculations give the flange component of $M_{S C \pm}(i, j)$ and $P_{S C \pm}(i, j)$ which will be called $M_{f 1 \pm}(i, j)$ and $P_{f 1 \pm}(i, j)$. The matrices $M_{f 1 \pm}(i, j)$ are too small since they do not include the contribution from multiple neutron scatterings in the flanges. An attempt was made to take multiple scattering into account by multiplying $M_{f 1 \pm}(i, j)$ by a factor, $C_{f 1}$. Experimental measurements, described in Section B, show that this gives a good fit between experiment and calculations if $C_{f 1} \simeq 2.5$ for the stainless steel flanges and $C_{f 1} \simeq 3.3$ for the aluminum flanges (see figs. VI-4, VI-5, VI-6). Rough calculations of double and triple scattering in a block $9 \times 18 \times 18 \mathrm{~cm}$, the approximate dimensions of the flanges, indicate that these values of $\mathrm{C}_{f 1}$ are reasonable. The calculated value of $C_{f l}$ for aluminum is slightly 
greater than that for stainless steel, and in both cases the calculated $C_{f 1}$ is not strongly dependent on the energy of the neutrons incident on the block, for neutrons in the energy range 1 to $15 \mathrm{MeV}$. Using the results of the rough calculations to justify using a $\mathrm{C}_{f 1}$ which is not dependent on the neutron energy, the values of $\mathrm{C}_{f l}$ used for the aluminum and stainless steel gas chambers were chosen to give the best fit of the calculated to the experimental scattering corrections described in section $B$. Thus the flange scattering corrections are given by the matrices $C_{f 1} M_{f 1 \pm}(i, j)$ and $P_{f 1 \pm}(i, j)$, with $C_{f 1}=3.3$ for the aluminum chamber and $C_{f l}=2.5$ for the stainless steel chamber.

4. Calculation of the Corrections for Scattering from the Tunne1 Wa11.

These calculations were done in a manner similar to that for the gas chamber flanges. The neutrons were attenuated on entering and.leaving the tunnel wall, with the attenuation cross section being taken as the total cross section with the forward elastic peak subtracted. The scattering geometry is similar to that shown in fig. VI-1, and $\mathrm{N}_{\mathrm{R} t}(i, j)$ are calculated with an equation like VI.9, with the sum over $d_{\mathrm{GC}}$ covering a section of the tunnel wall about $10 \mathrm{ft}$. long and $2 / 3 \mathrm{ft}$. thick. The tunnel wall material (concrete) was taken to be $\mathrm{SiO}_{2}$ with a density of 3 , and with the neutron scattering cross section being an average of those for oxygen and silicon, weighted in the ratio $2: 1$. The number of scattering nuclei/cm ${ }^{3}$ was taken to be the total number of silicon and oxygen nuclei/cm ${ }^{3}$. The final matrices for the tunnel scattering will be labeled $\mathrm{M}_{\mathrm{TU} \pm}(i, j)$ and $\mathrm{P}_{\mathrm{TU}}(i, j)$. 
The tunnel scattering calculations had no corrections made for multiple scattering of neutrons in the tunnel wa11. Multiple scattering in the tunnel wall is assumed to be a small effect because most of the multiply scattered neutrons will be cut off by the coincidence circuit timing. This arises because the flight paths involved in scattering from the tunnel wall were generally quite long (several feet), and multiply scattered neutrons tend to have low energies. The contribution from $\gamma$ - rays from inelastic scattering is also assumed to be little affected by multiple scattering, primarily because of the large uncertainties in the $\gamma$-ray contribution because of the poorly known $\left(n, n^{\prime} \gamma\right) \gamma$-ray multiplicities.

5. Final Form of the Scattering Correction Matrices, and Their Uncertainty.

The total scattering correction matrices, used in Chapter IV, are

$$
\begin{aligned}
M_{S C \pm}(i, j)= & M_{C Y \pm}(i, j)+C_{f 1} M_{f 1 \pm}(i, j)+M_{T U \pm}(i, j) \\
P_{S C \pm}(i, j)= & {\left[P_{C Y \pm}(i, j) M_{C Y \pm}(i, j)+C_{f 1} P_{f 1 \pm}(i, j) M_{f 1 \pm}(i, j)+\right.} \\
& \left.P_{T U \pm}(i, j) M_{T U \pm}(i, j)\right] / M_{S C \pm}(i, j) .
\end{aligned}
$$$$
\text { VI. } 1.0
$$

The matrices VI.10 were used as shown in chapter IV to correct the polarimater data for the scattering of neutrons by the gas chamber cylinder and flanges and by the tunnel wall. It should be noted that the matrices VI.10 depend on the central scattering angle of the side-detector pair, and thus the analysis of polarization data generally required four sets of 
matrices, one set for each side-detector pair.

The uncertainty in the matrices VI.I0 is assumed to be $30 \%$. This is based on a comparison of the experimental and theoretical results to be made in section $B$ of this chapter, and includes an estimate of the errors made with some of the approximations used in the calculations. The estimate of $30 \%$ error gives the results of eq. IV.30 in chapter IV, and is felt to be more likely an over-than an under-estimate of the error for the polarimeter and tunnel scattering corrections.

\section{B. Measurement of the Corrections for Polarimeter}

$\underline{\text { Scattering for } 14.7 \mathrm{MeV} \text { and } 2.86 \mathrm{MeV} \text { Neutrons }}$

1. Results of the Measurements.

The corrections for polarimeter scattering were measured for $14.7 \mathrm{MeV}$ neutrons from the $t(d, n) \mathrm{He}^{4}$ reaction, and are plotted in figs. VI-4 and VI-5. The graphs show the relative $\mathrm{He}^{4}$ recoil spectrum, with the 14.7. MeV peak normalized to unity and the $\mathrm{He}^{4}$ recoil energy plotted as apparent neutron energy. Fig. VI-4 shows the results for the aluminum gas chamber, for side-detectors at central neutron scattering angles of 113 degrees and 155 degrees with a gas counter to side-detector distance of 30 inches. The theoretical calculations for the response to $14.7 \mathrm{MeV}$ neutrons are also plotted, with $\mathrm{C}_{\mathrm{f} 1}=3.3$. Fig. VI-5 shows the results for the stainless steel gas chamber, for side-detector angles of $113,127,141$, and 155 degrees, and a gas-counter to sidedetector distance of 30 inches. The calculated responses, with $C_{f l}=2.5$, are also plotted in fig. VI-5. The experimental results 


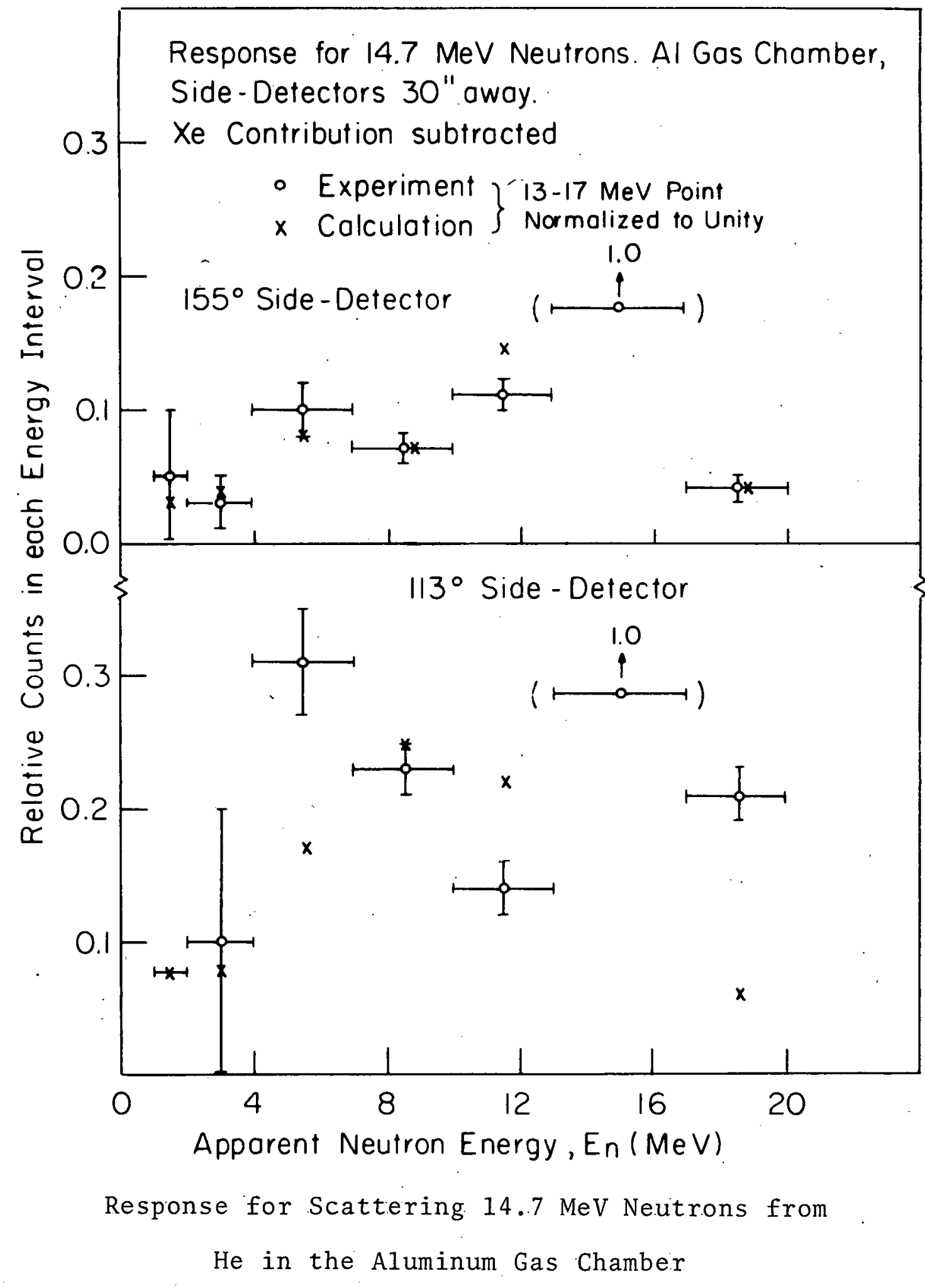

Fig. VI-4. 


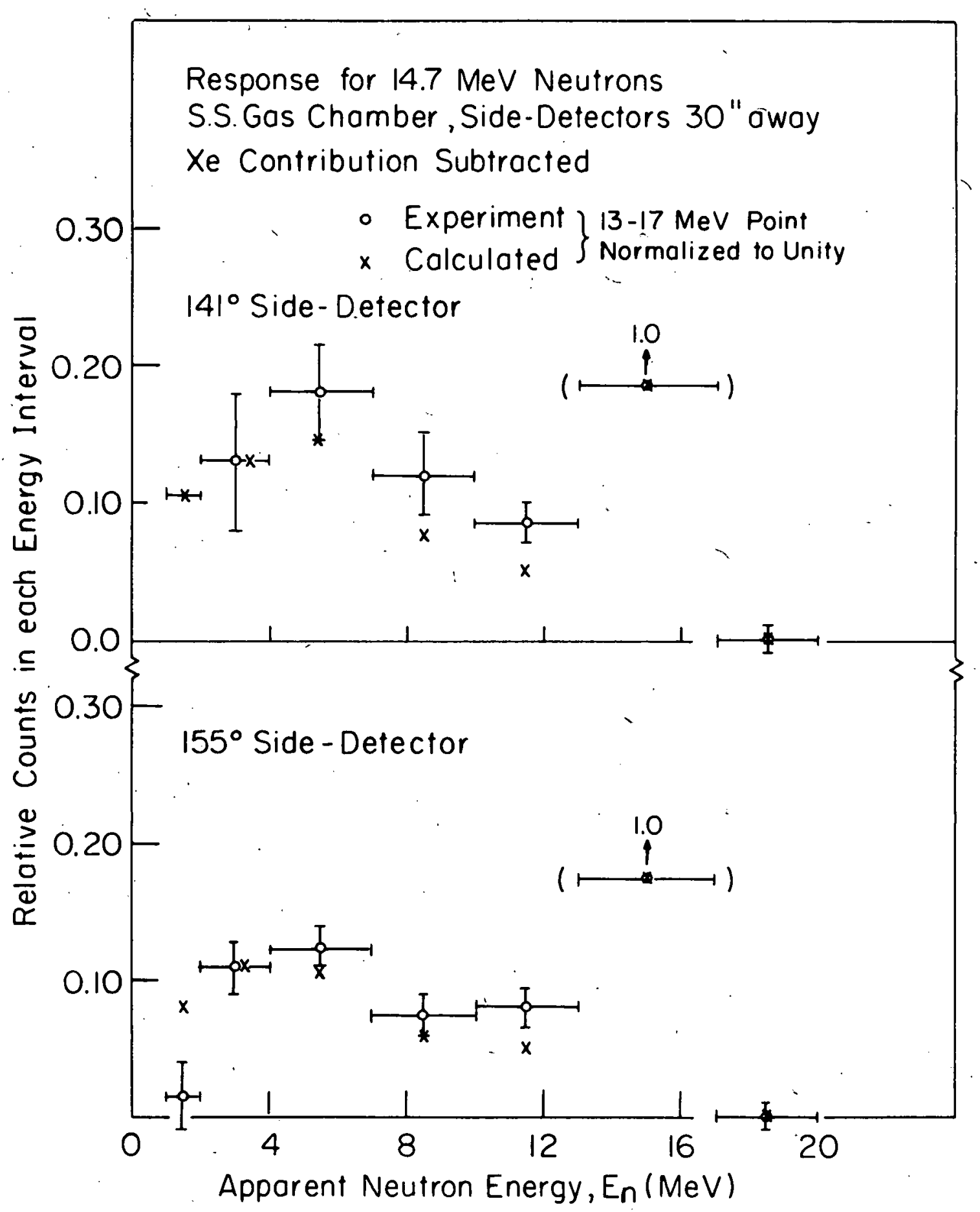

Response for Scattering $14.7 \mathrm{MeV}$, Neutrons from He in the Stainless Steel Gas Chamber Fig. VI $-5 a$ 


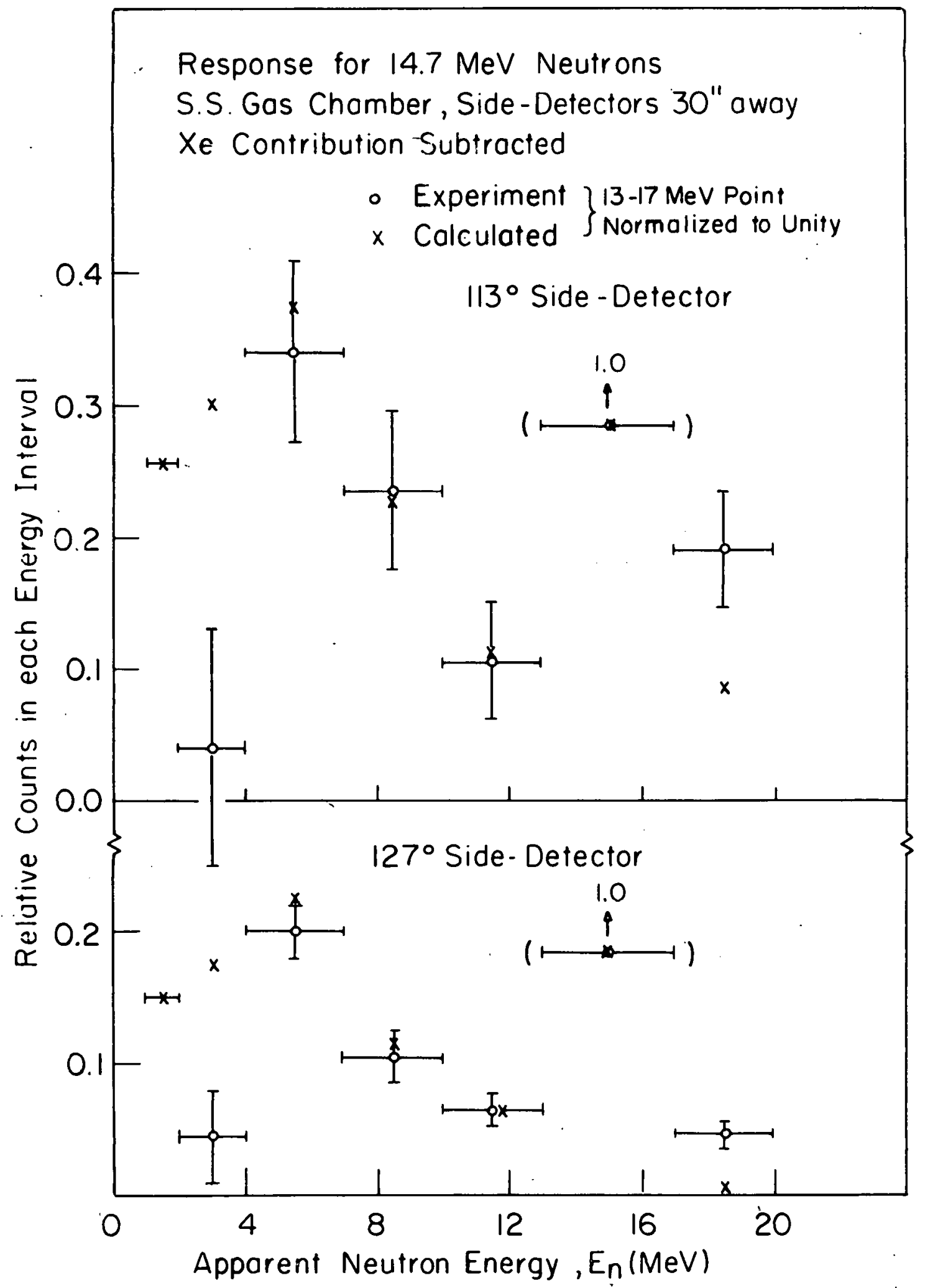

Response for Scattering 14..7 MeV Neutrons. from He in the Stainless Steel Gas Chamber

Fig. VI $-5 b$ 
shown in figs. VI-4 and VI-5 were used to find empirical values for $\mathrm{C}_{\mathrm{fl}}$ for the aluminum and stainless steel gas chambers.

The corrections for polarimeter scattering of $2.86 \mathrm{MeV}$ neutrons from the $d(d, n) H^{3}$ reaction are plotted in fig. VI-6, along with the calculations with $C_{f 1}=2.5$. The results are shown for the stainless steel gas chamber with side-detectors at 113 and 155 degrees, 30 inches from the gas chamber, and with a side-detector at 67 degrees, 20 inches from the gas chamber. These results indicate that the value of $\mathrm{C}_{f 1}$ obtained from the $14.7 \mathrm{MeV}$ neutrons is still quite accurate for 2.86 $\mathrm{MeV}$ neutrons, and substantiate the rough calculations which indicated that $\mathrm{C}_{f l}$ should not depend strongly on the neutron energy.

By placing a second steel cylinder around the stainless steel gas chamber and finding the difference in the response with and without the extra cylinder, it was possible to measure the scattering corrections from the gas chamber cylinder alone. The results for $14.7 \mathrm{MeV}$ neutrons are shown in fig. VI-7, along with the calculated response.for the stainless steel cylinder. The results are for side-detectors at 113, 127 and 155 degrees, all 30 inches from the stainless steel gas chamber. Since there is little multiple scattering in the gas chamber cylinder wall these results are a good check on the accuracy of the scattering calculations.

The calculated results plotted in figs. VI- 4 to. VI-7 are

$$
\frac{N_{R+}(i, j)+N_{R-}(i, j)}{2 \bar{S}_{H e}\left(\theta_{0}, E_{n}(j)\right)} \equiv \frac{N_{L^{+}}(i, j)+N_{L-}(i, j)}{2 \bar{S}_{H e}\left(\theta_{0}, E_{n}(j)\right)}
$$

with the experimental resolution of the gas-counter folded into 


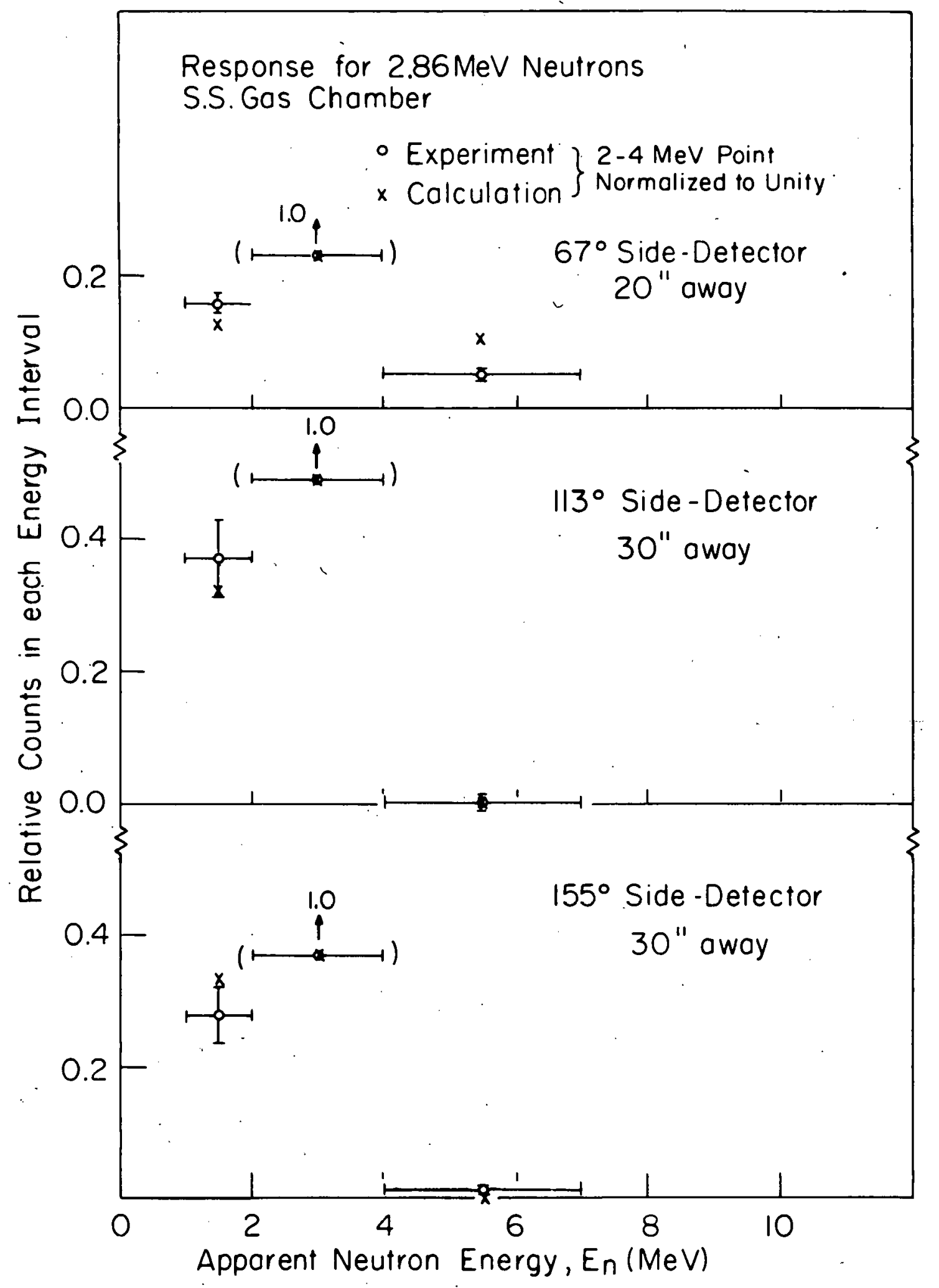

Response for Scattering $2.86 \mathrm{MeV}$. Neutrons from $\mathrm{He}$ in the Stainless Steel Gas Chamber

Fig. VI- 6 


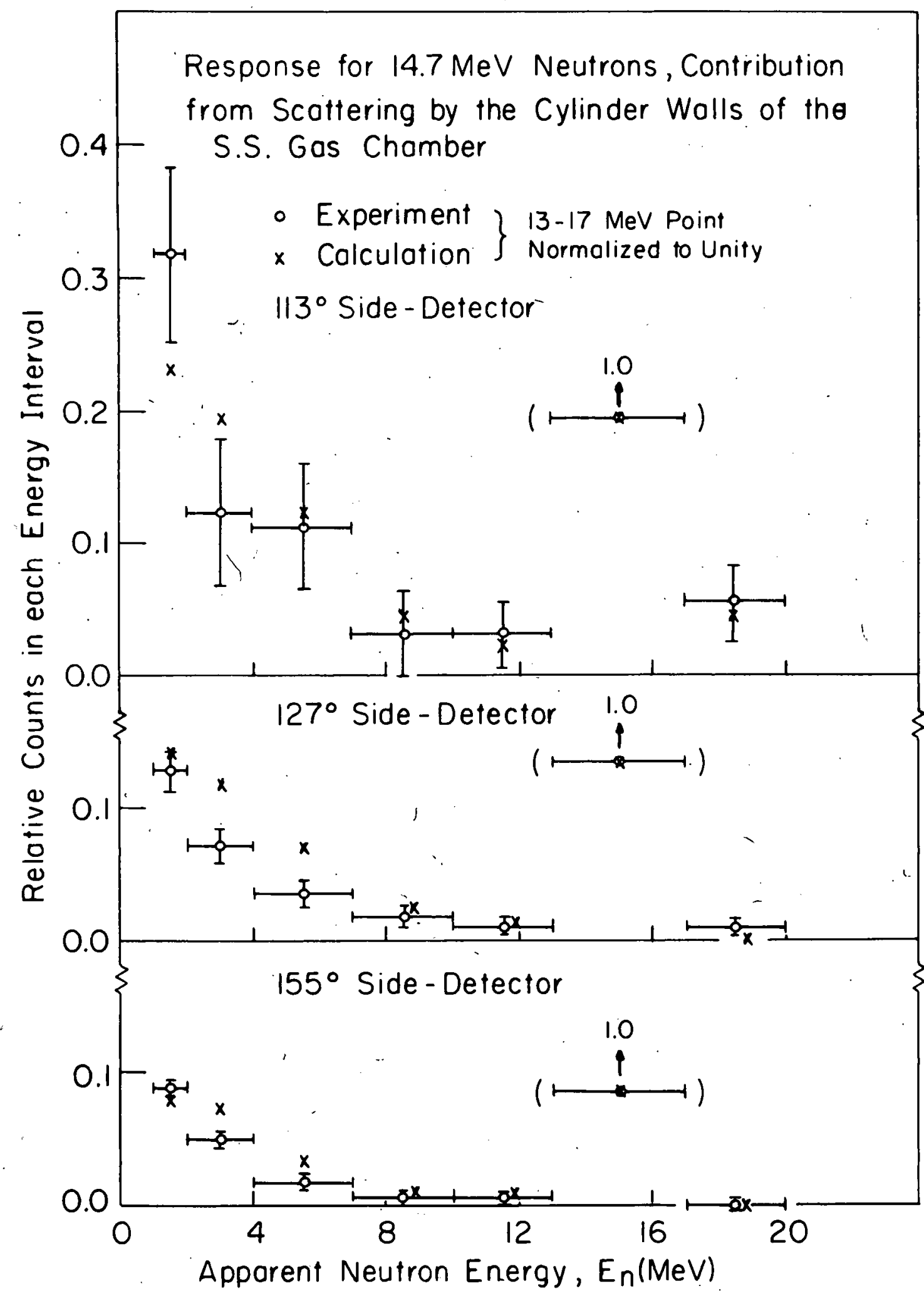

Cylinder Component of the Response for Scattering $14.7 \mathrm{MeV}$ Neutrons from He in the Stainless Steel Gas Chamber Fig. VI -7 
the calculated spectra. In eq. VI.11 $\mathrm{E}_{\mathrm{n}}(\mathrm{j})=14.7 \mathrm{MeV}$, as is required, and the index $i$ describes the $\mathrm{He}^{4}$ recioil spectrum. The values of $\mathrm{N}_{\mathrm{R}_{ \pm}}(\mathrm{i}, j)$ are summed results of the cylinder and flange scattering, except for fig. VI-7 where only the cylinder contribution is taken.

The polarimeter response for $14.7 \mathrm{MeV}$ neutrons has an appreciable contribution from scattering from the xenon in the gas chamber. Fig. VI-8 shows the measured response for a filling of 80 psia of xenon. The measured response for a filling of 80 psia of xenon and 720 psia of helium is shown in fig. VI-9. These results are for side-detectors at $113,127,141$ and 155 degrees, located 30 inches from the stainless steel gas chamber. The results of fig. VI-5 are the differences of the plots in fig. VI-9 and fig. VI-8.

The polarization data presented in this thesis have had no corrections made for the xenon scattering. The results in fig. VI-8 show that xenon scattering is significant only for high energy incident neutrons, and produce corrections at low apparent neutron energies. Since none of the polarization measurements gave significant data at low neutron energies ( $<3 \mathrm{MeV}$ ) while also having high energy (> $10 \mathrm{MeV}$ ) neutrons present the absence of xenon scattering corrections has no effect on the final polarization measurements presented here.

\section{Method of Making the Measurements}

The $14.7 \mathrm{MeV}$ neutrons from $t(d, n) \mathrm{He}^{4}$ and the $2.86 \mathrm{MeV}$ neutrons from $d(d, n) \mathrm{He}^{3}$ were obtained from the Cockroft-Walton accelerator of the MIT Nuclear Engineering Dept. The physical layout of the accelerator, the neutron collimator, and the 


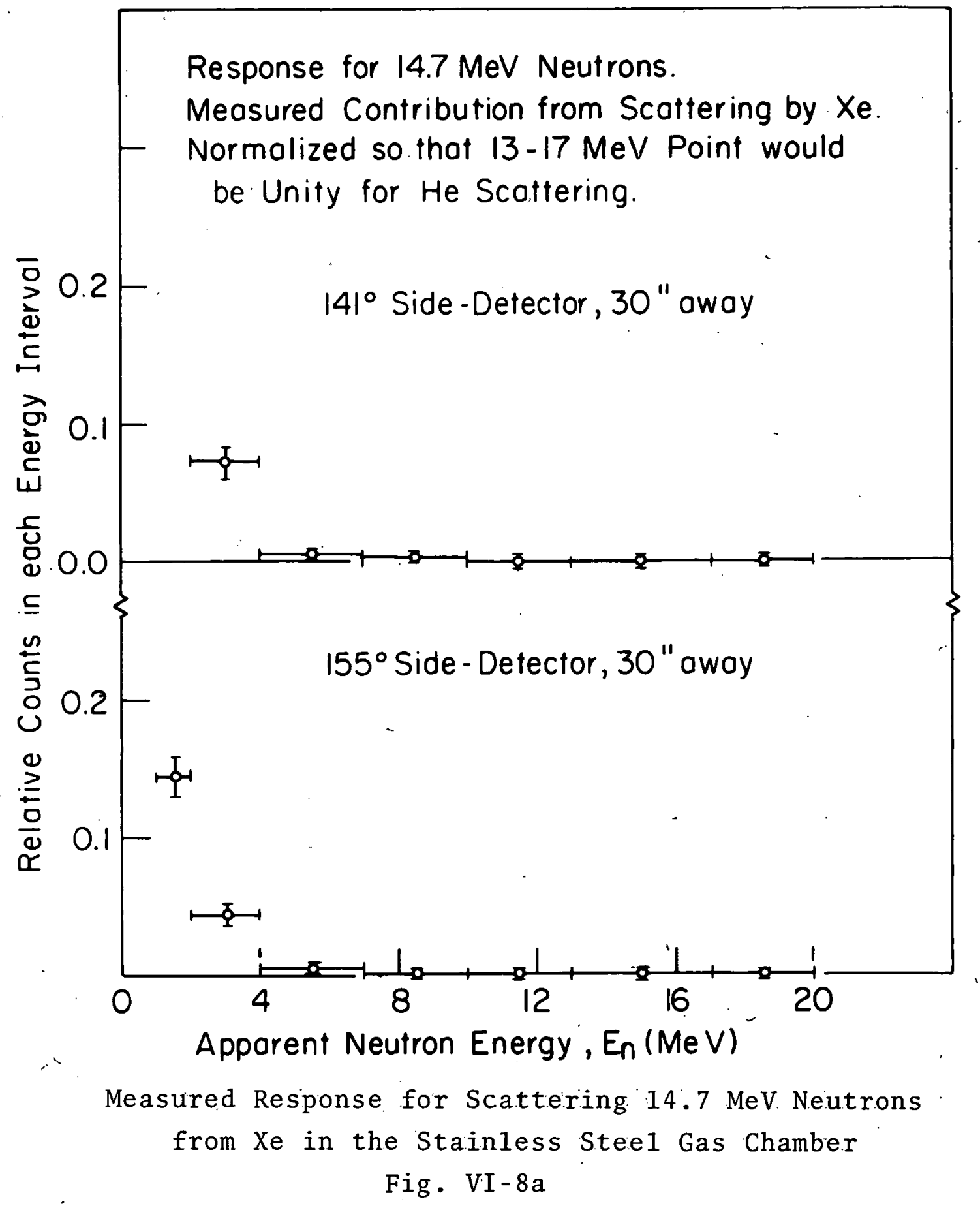




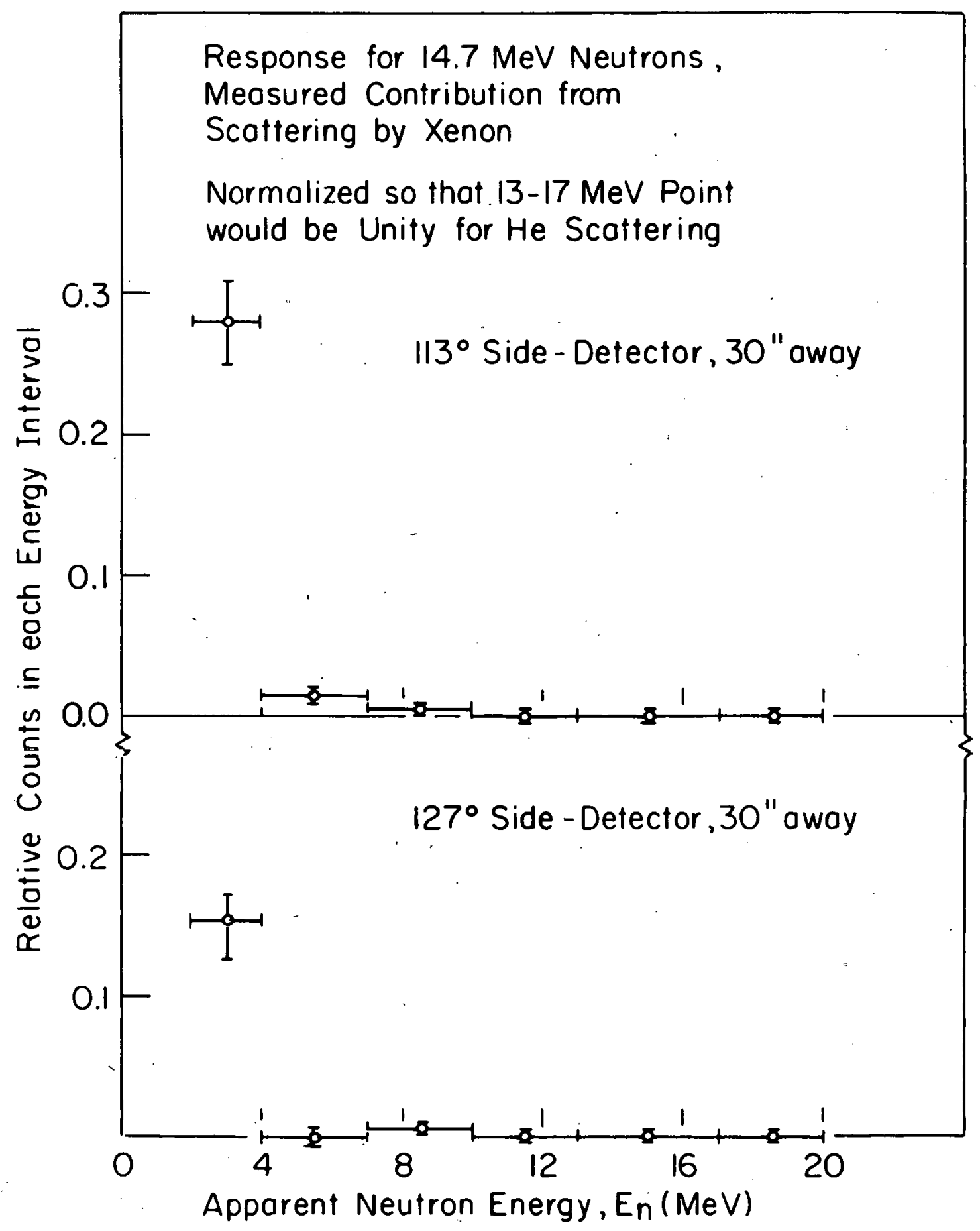

Measured Response for Scattering 14.7 MeV Neutrons from $\mathrm{Xe}$ in the Stainless Steel Gas Chamber.

Fig. VI-8b 


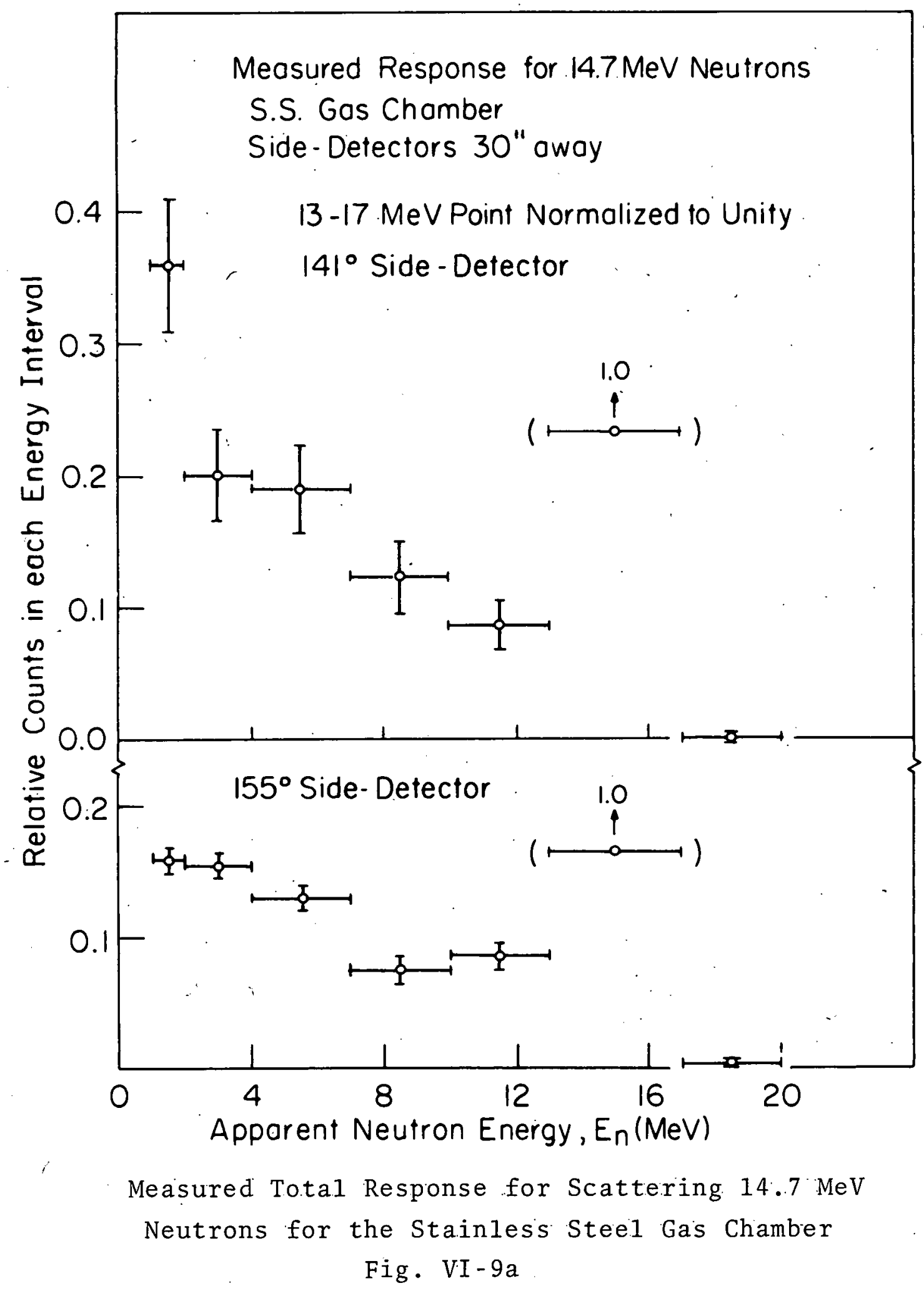




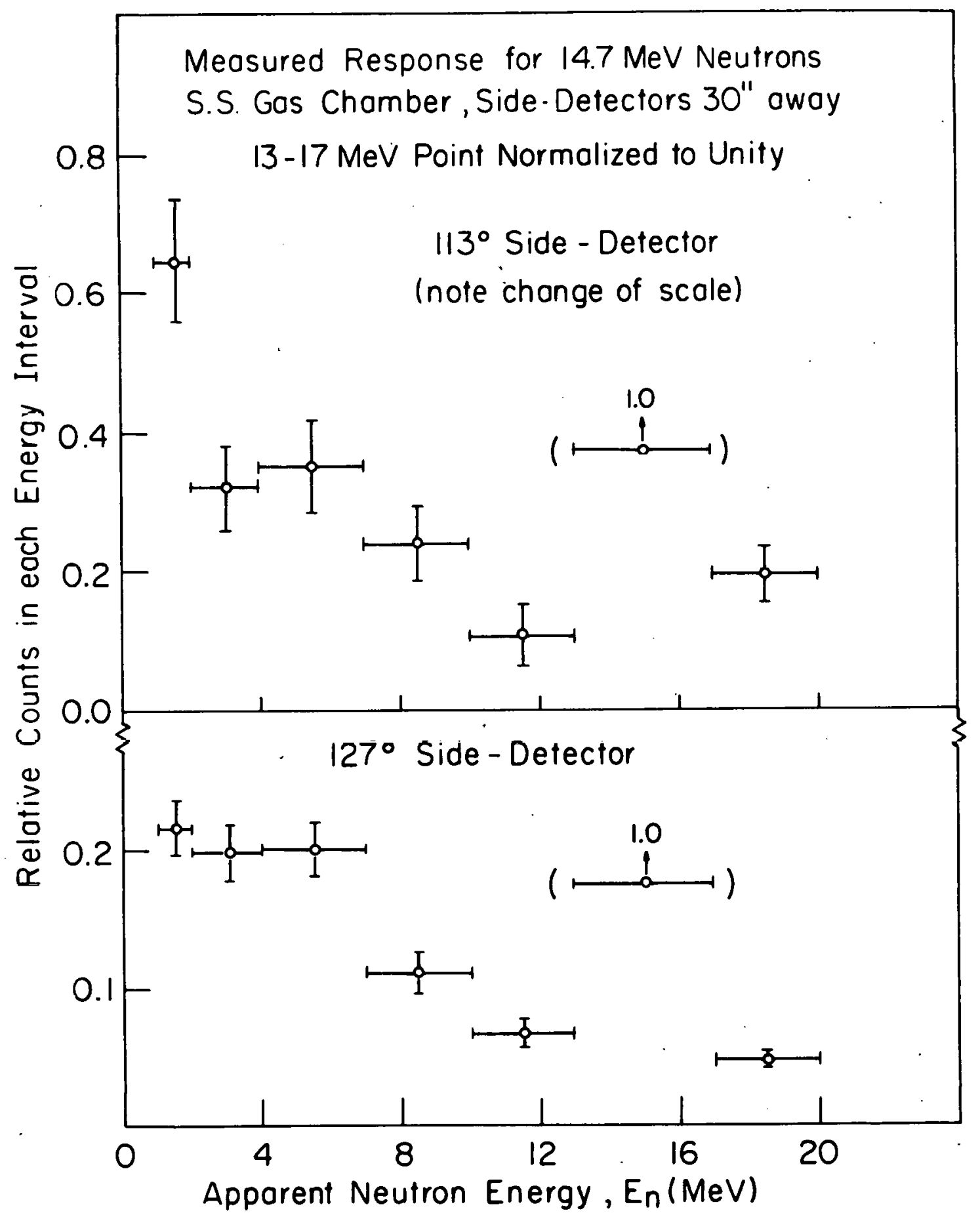

Measured Total Response for Scattering $14.7 \mathrm{MeV}$ Neutrons for the Stainless Steel Gas Chamber

Fig. VI-9b. 
polarimeter is shown in fig. II-7. The neutron beam was collimated to about $61 / 2 "$ diameter, at the gas chamber. A $\mathrm{BF}_{3}$ tube in the accelerator room was used to monitor the relative yield for each run. The forward (or zero degree) neutron beam was used, which gave a zero polarization for the beam and thus enabled scattering corrections to be measured by using only one side-detector at a given angle. The scattering corrections for the $2.86 \mathrm{MeV}$ neutrons were measured with the direct neutron beam. The measurements with the $14.7 \mathrm{MeV}$ beam were made with $5^{\prime \prime}$ of wax in the beam to filter out low energy neutrons, and it is estimated that under these conditions the region 1-12 MeV contained less than 5\% as many neutrons as did the $14.7 \mathrm{MeV}$ peak.

The accuracy with which the corrections for $14.7 \mathrm{MeV}$ neutrons can be measured depends on the purity of the beam. For the measured scattering corrections there are about $50 \%$ as many apparent neutrons in the interval $1-12 \mathrm{MeV}$ as are observed in the $14.7 \mathrm{MeV}$ peak. If we neglect the energy dependence of the polarimeter detection efficiency, then this' requires that $95 \%$ of the neutrons incident on the polarimeter should be in the $14.7 \mathrm{MeV}$ peak, if we wish to measure the scattering corrections with less than $10 \%$ systematic error from contamination.

Because of the variation with energy of the transmission of neutrons by wax it is possible, for a given apparent neutron energy interval, to separate the scattering correction component of the $14.7 \mathrm{MeV}$ neutrons and the direct component from actual neutrons in this energy interval. The first component will be attenuated as the $14.7 \mathrm{MeV}$. neutrons, and the second as neutrons 
in the given interval. This procedure neglects scattering correction contributions from al1 except the $14.7 \mathrm{MeV}$ neutrons. The scattering corrections were measured first with the direct neutron beam, and then with the beam attenuated by $11 / 4^{\prime \prime}, 5^{\prime \prime}$, and $15^{\prime \prime}$ of wax. The wax was about half way between the neutron source and the polarimeter, with collimators on both sides, so good geometry attenuation of the neutron beam should result. For reference, the neutron transmission coefficient for $5^{\prime \prime}$ of wax is plotted against neutron energy. in fig. VI-10.

The results of the attenuation tests are shown in semi-1og plots for four apparent neutron energy intervals in fig. VI-11. The plots show the number of detected neutrons appearing in the energy interval per unit count of the $\mathrm{BF}_{3}$ monitor, this quantity being plotted on a log scale against the thickness of wax in the neutron beam. The run with the direct beam has been normalized to unity in each interval. The curves show the expected transmission for $14.7 \mathrm{MeV}$ neutrons, and for neutrons in the interval shown on the individual plots.

On the basis of the results of fig. VI-11 it is estimated that $5^{\prime \prime}$ of wax has sufficiently lowered the relative number of neutrons below about $7 \mathrm{MeV}$ to give meaningful scattering correction measurements. For the region above $7 \mathrm{MeV}$ neutron energy the attenuation is too close to that for $14.7 \mathrm{MeV}$ neutrons to give a useful measurement of the components, within'the counting statistics used for the given runs.

A second test, useful mainly for higher energy neutrons, was made by measuring the pulse height spectrum in a $1 / 8$ " $x 2^{\prime \prime}$ diameter piece of plastic scintillator (Pilot B) mounted on an RCA 6810A photomultiplier. The measurements were made with no 


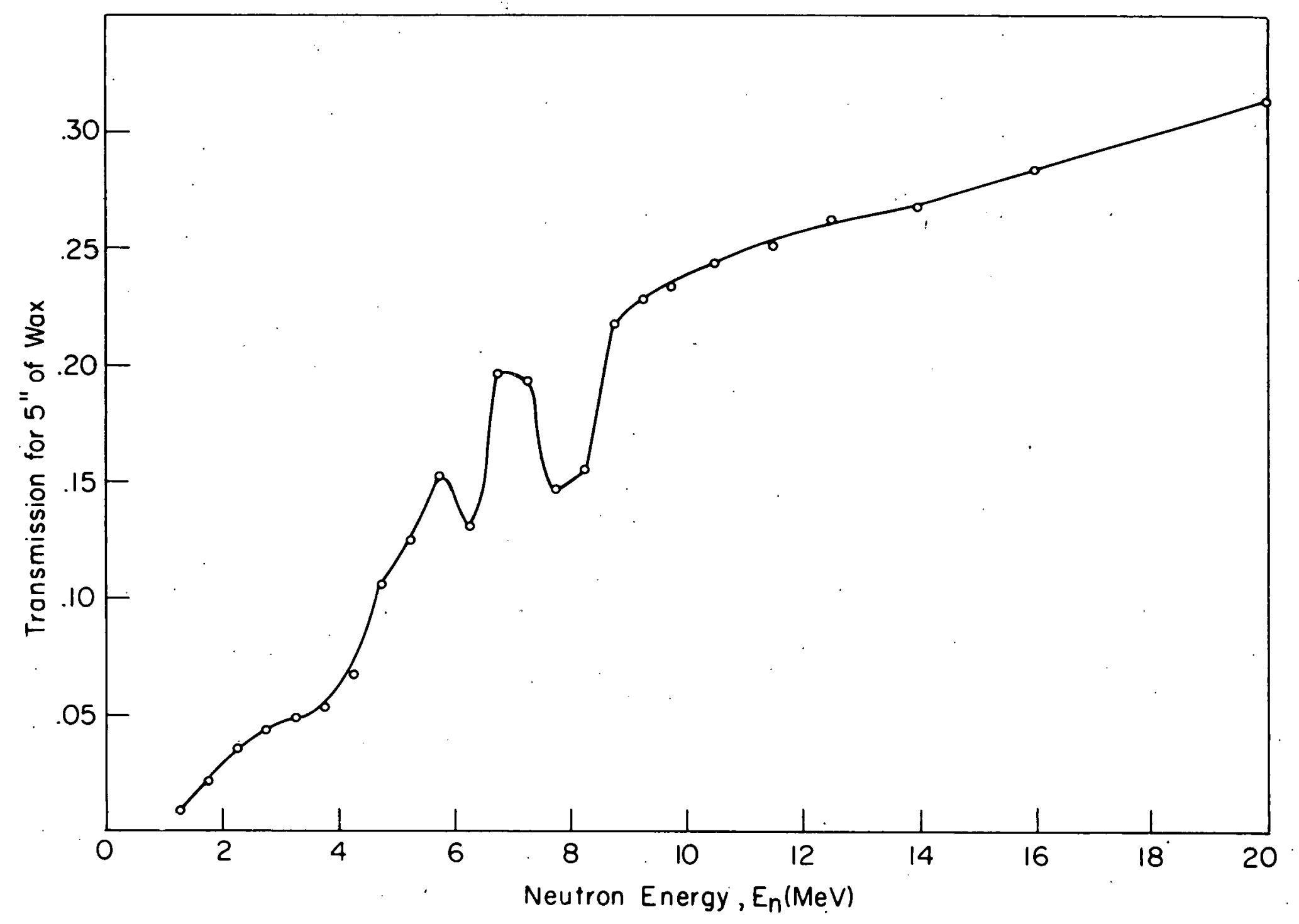

Neutron Transmission by $5^{\prime \prime}$ of Wax

Fig. VI-10. 


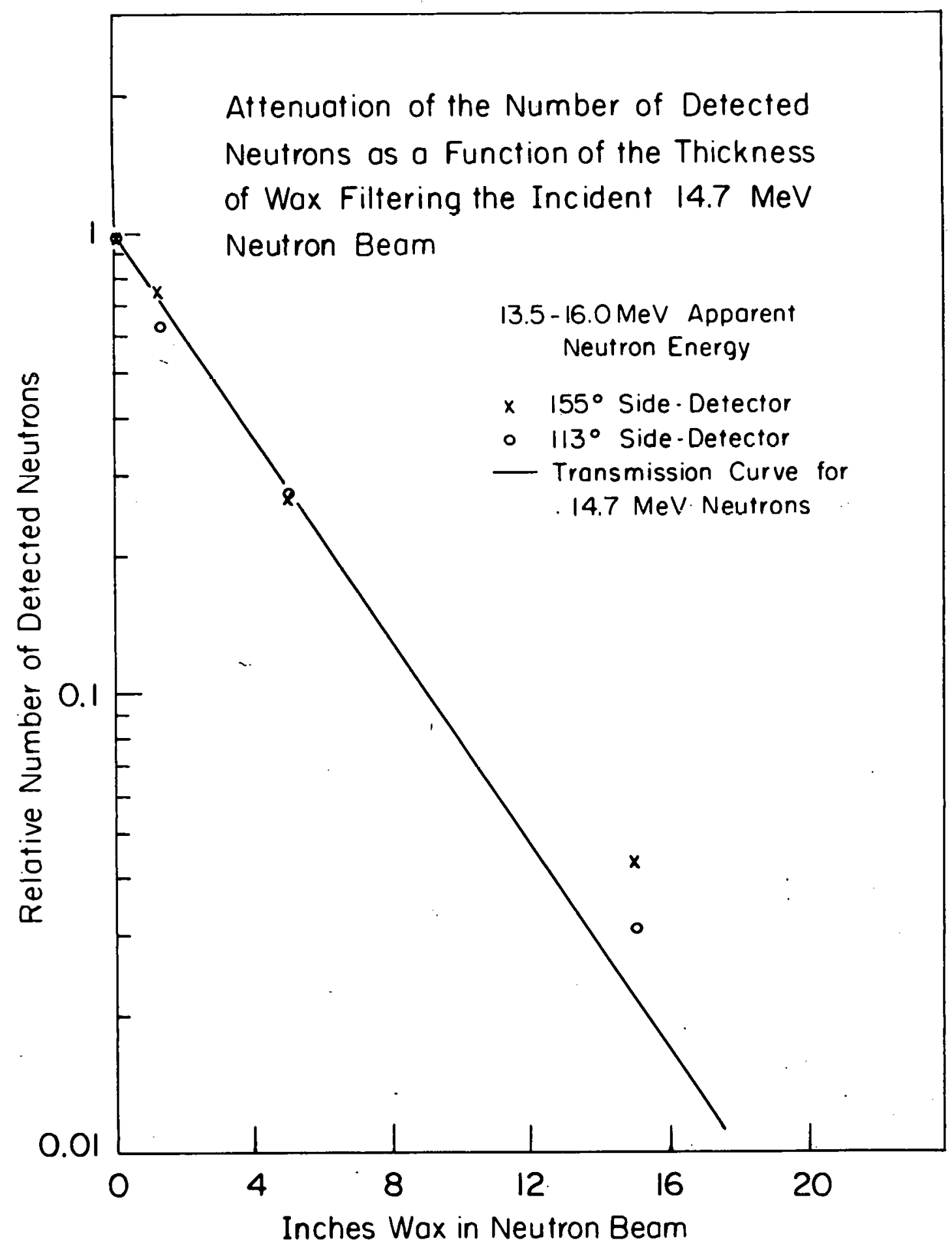

Attenuation of Polarimeter Response as a Function of Thickness of Wax in the $14.7 \mathrm{MeV}$ Neutron Beam

Fig. VI-11a 


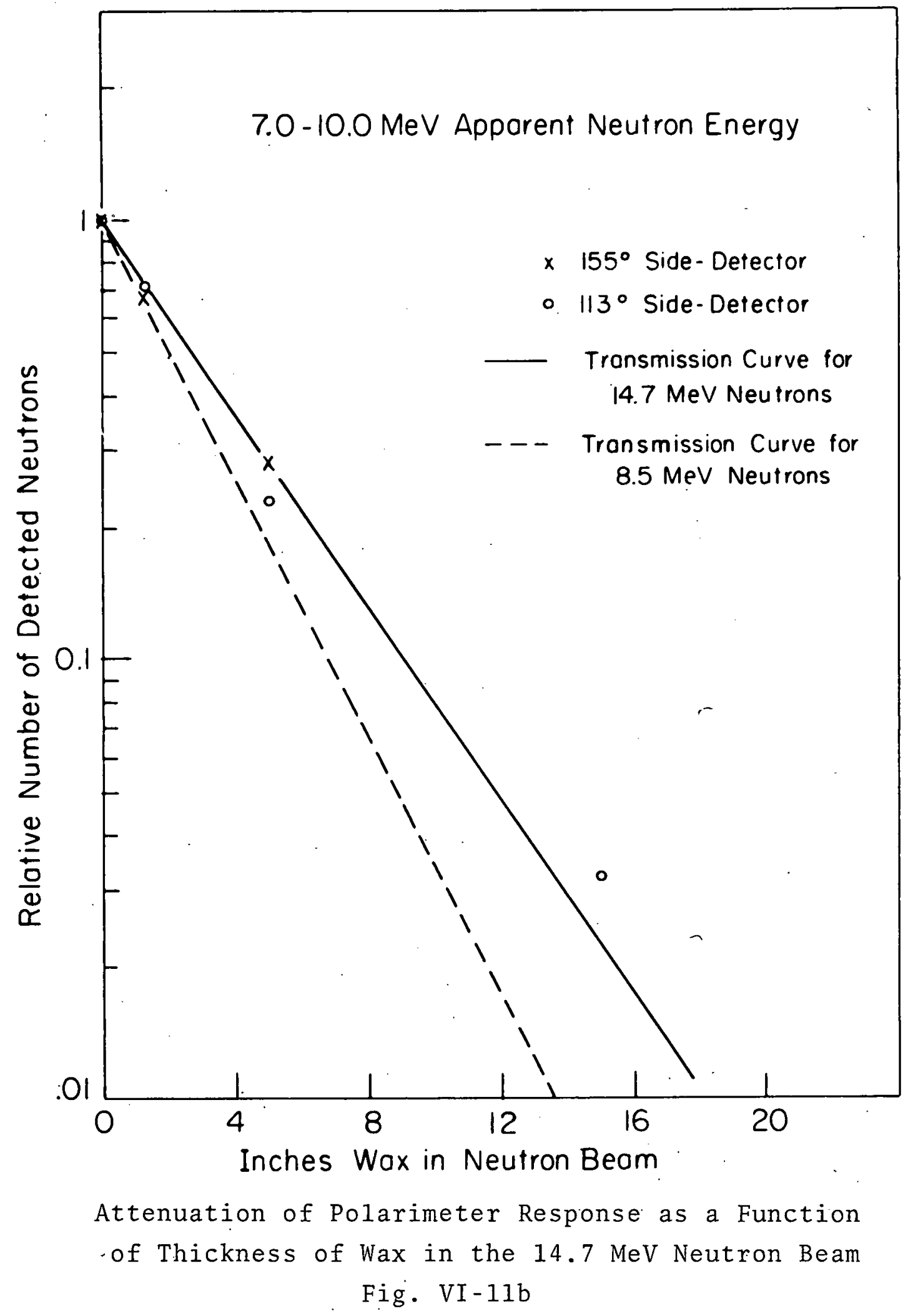




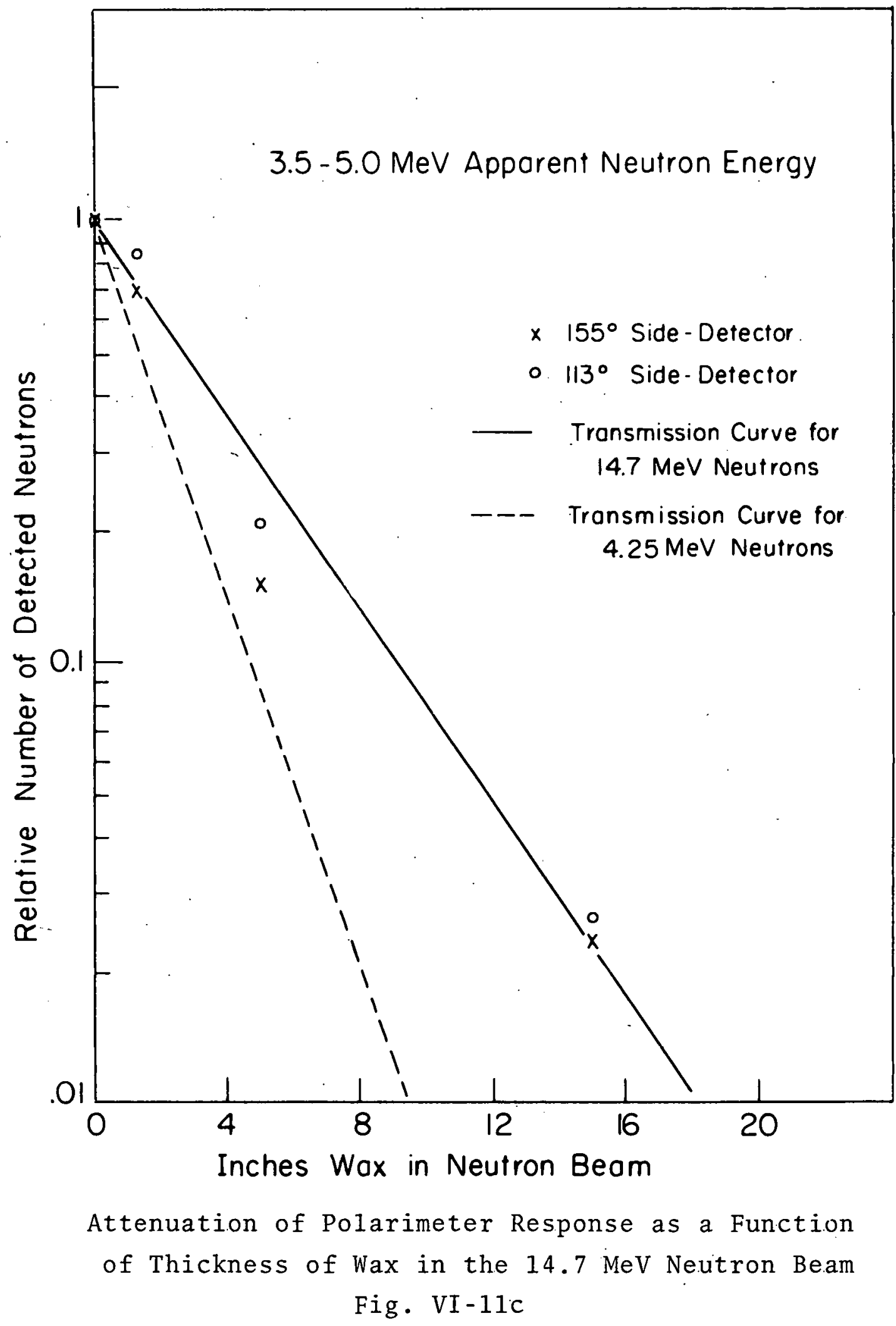




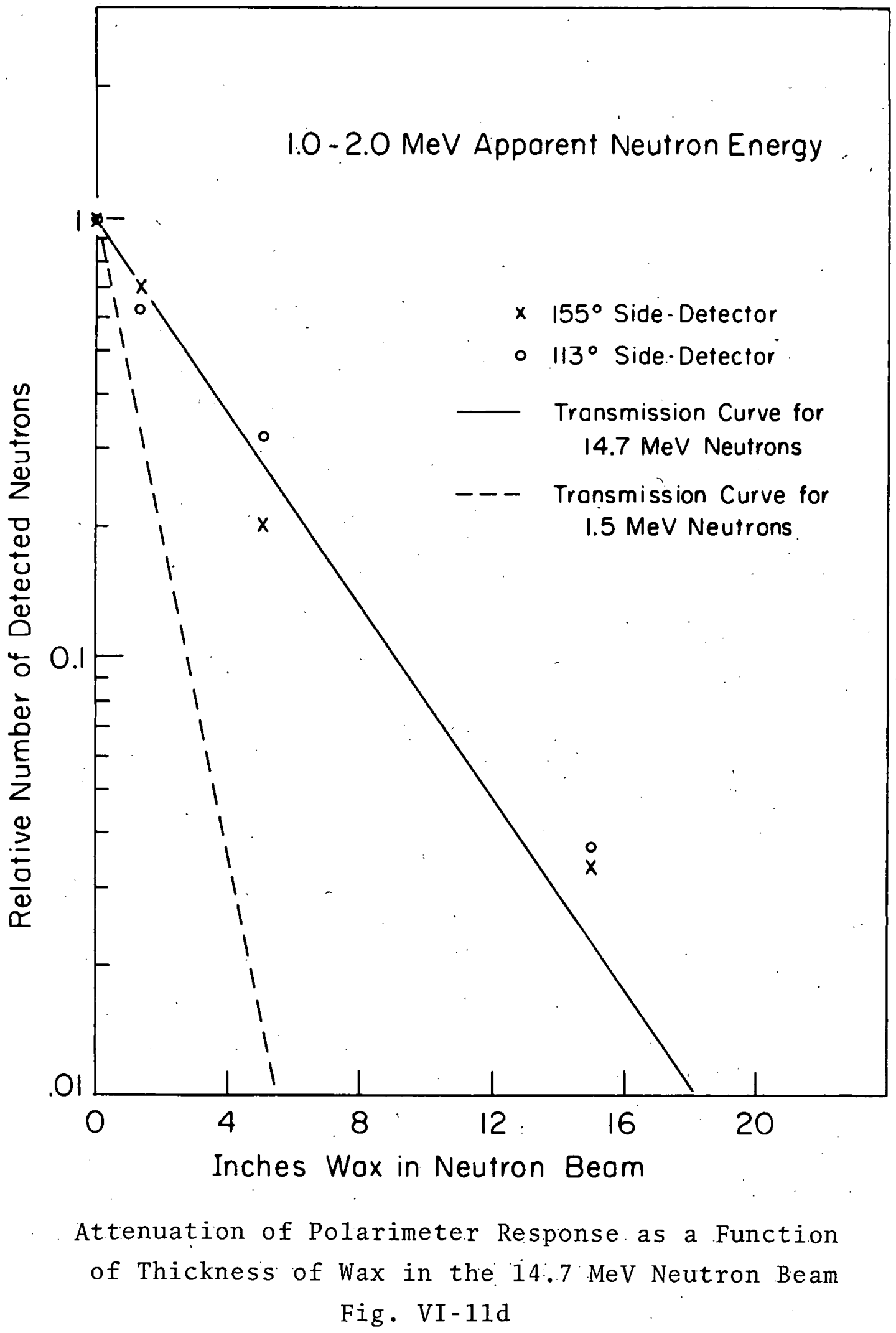


wax filtering the collimated $14.7 \mathrm{MeV}$ neutron beam. The pulse height spectrum given by this piece of plastic in the $14.7 \mathrm{MeV}$ neutron beam is shown in fig.VI-12, where it is compared with calculations and measurements by Batchelor et al. (56) for 14 $\mathrm{MeV}$ neutrons incident on a $21 / 2^{\prime \prime} \times 2^{\prime \prime}$ diameter volume of NE213 liquid scintillator. Their source of neutrons was the $t(d, n)$ $\mathrm{He}^{4}$ reaction, and their measurements were made with a time of flight method, and with a pulse shape discrimination method.

The response of the liquid scintillator and the plastic are not expected to be significantly different.

At the time the tests for beam purity were made only the $1 / 8$ " plastic disc was available. To check whether edge effects would allow the response of Batchelor et al for a $21 / 2$ " thick cylinder to be applied to the $1 / 8^{\prime \prime}$ disc tests were later made with a $2 "$ thick $x 2^{\prime \prime}$ diameter piece of plastic scintillator. The response of the $2 "$ thick plastic cylinder could not be used in fig. VI-12 as the collimation system had been dismantled before such tests could be made. Therefore the response for the $1 / 8^{\prime \prime}$ plastic and the $2 "$ thick plastic were compared by measuring their response when irradiated in the $C-W$ target room. The results indicate that the $1 / 8^{\prime \prime}$ plastic has a slightly decreasing response at the higher pulse heights when compared to the $2^{\prime \prime}$ plastic, this result probably arising from the larger edge effects in the smaller plastic. Thus the 1/8" plastic would give a response for a pure $14.7 \mathrm{MeV}$ neutron beam which is closer to the response for a contaminated beam, and any conclusions about the beam composition made from fig. VI-12 will overestimate the contamination.

From the experimental response for the $1 / 8^{\prime \prime} \mathrm{plastic}$ and the two curves for calculated response shown in fig. VI-12 it is 

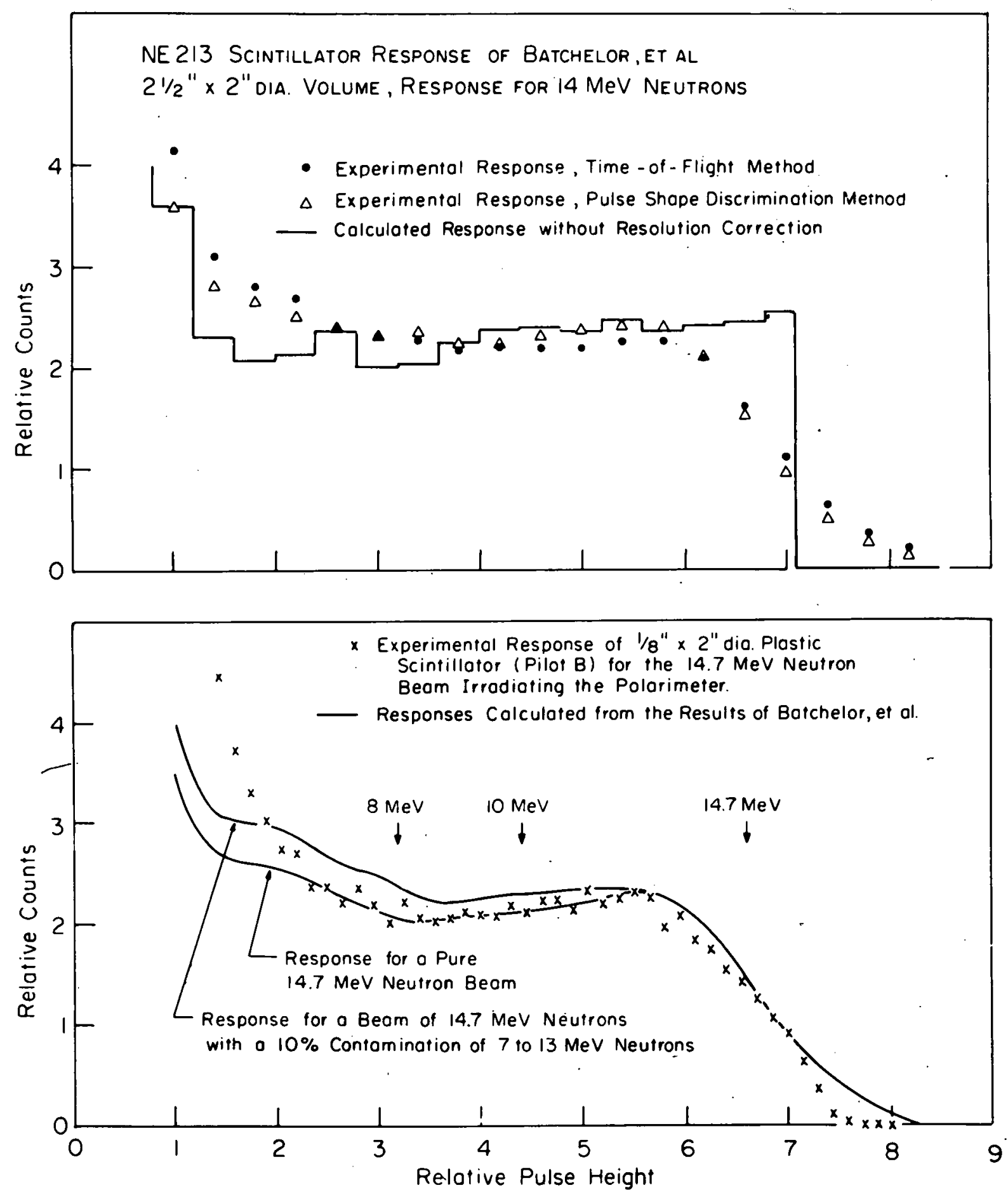

Response of a Plastic Scintillator to the $14.7 \mathrm{MeV}$ Neutron Beam Fig. VI-12 
estimated that the contamination of the $14.7 \mathrm{MeV}$ neutron beam is definitely less than $10 \%$, and probably less than $5 \%$, for neutrons in the range 7 to $13 \mathrm{MeV}$. Since most neutrons in the range 7 to $13 \mathrm{MeV}$ come from the $14.7 \mathrm{MeV} t(\mathrm{~d}, \mathrm{n})$ neutrons scattering in the tritium target assembly, the contamination was also estimated by calculating the amount of this scattering. The calculations yield about $3 \%$ for the range 7 to $13 \mathrm{MeV}$ neutron energy, and provide a check on the experimental method.

The scattering correction measurements were al1 made with side-detector thresholds of about $0.75 \mathrm{MeV}$ and with coincidence circuit timing of $8 \mathrm{~ns}$ to $60 \mathrm{~ns}$. To determine the effect of timing changes on the scattering corrections several runs were made with different timings. If we label the timing hy the shortest delay, 8 ns for the case just mentioned, then we can plot the relative response in a given neutron energy interval as a function of delay time. Fig. VI-13 shows such a plot for four neutron energy intervals for a side-detector at 155 degrees, 30" from the stainless steel gas chamber. The plots show a negligible dependence of the measured response on the delay timing, for the shortest delay being in the range of about 0 ns to $20 \mathrm{~ns}$. This result should apply to the other side-detectors also, and is taken to indicate that the scattering corrections are not critically dependent on the timing since all measurements reported here were made in this range.

C. Corrections for Scattering Within the Photoneutron Target

The final correction to be made to the polarization data comes from internal scattering in the photoneutron target. There are two aspects of this scattering. First, neutrons 


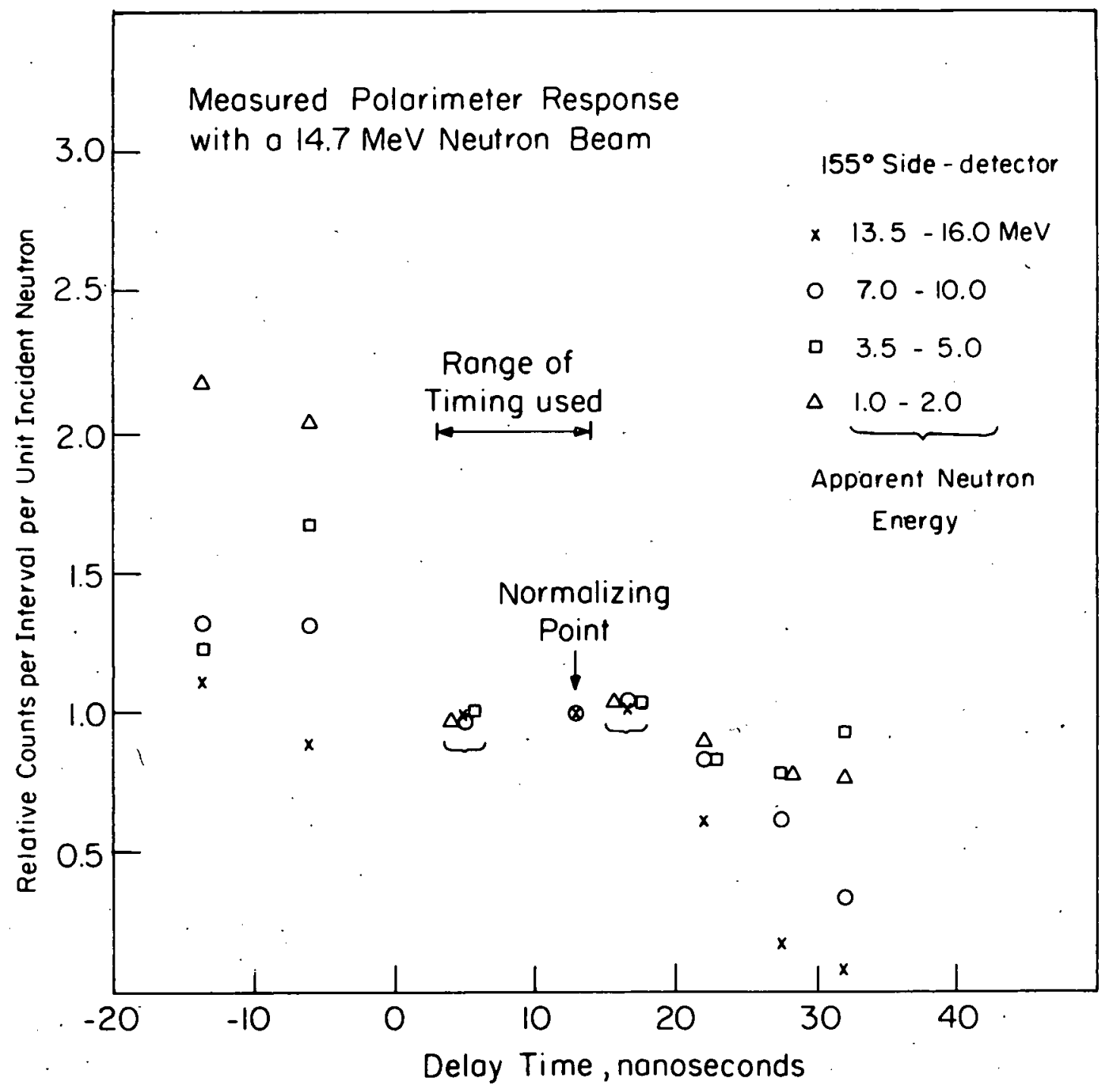

Polarimeter Response as a Function of Coincidence Circuit Delay Timing, Using a $14.7 \mathrm{MeV}$ Neutron Beam

Fig. VI -13 
produced in the direction of the polarimater may be scattered. This reduces the intensity of the photoneutron beam but does not affect its polarization. Second, the neutrons produced in directions other than that of the polarimeter may be scattered toward the polarimeter. In this case both the intensity and the polarization of the photoneutron beam are changed. The latter type of scattering also mixes neutrons of different energy, since higher energy neutrons may scatter off a light nucleus and lose sufficient energy to contribute to the neutrons in a lower energy bin.

To find the target scattering corrections for production at angle $\theta_{0}$ it is necessary to calculate three numbers for each neutron energy interval. $\mathrm{N}_{t d}(i)$ is the number of neutrons in the $i$ 'th energy interval, produced at angle $\theta_{0}$ and leaving the target without scattering. $N_{t}$ ind $(i)$ is the number of neutrons in the $i^{\prime}$ th energy interval which leave the target heading for the polarimeter, but have been scattered within the target; $\mathrm{P}_{t}$ ind $(\mathrm{i})$ is the polarization of the neutrons, $N_{t}$ ind $(i)$. The target scattering corrections can be subtracted by using eq.IV.37 of chapter IV with

$$
\begin{aligned}
& k_{\text {TS }}=\frac{N_{t \text { ind }}(i)}{N_{t d}(i)+N_{t \text { ind }}(i)} \\
& \mathrm{P}_{\text {TS }}=\mathrm{P}_{\text {Tind }}(i)
\end{aligned}
$$$$
\text { VI. } 12
$$

The photoneutron targets used to obtain the data for this thesis were generally one of two shapes, either a spherical shell or a solid cylinder. Accordingly, computer programs were developed to calculate scattering corrections for these two geometrical target shapes. The geometry used for the calculations for a cylindrical target is shown in fig. VI-14. We have for 


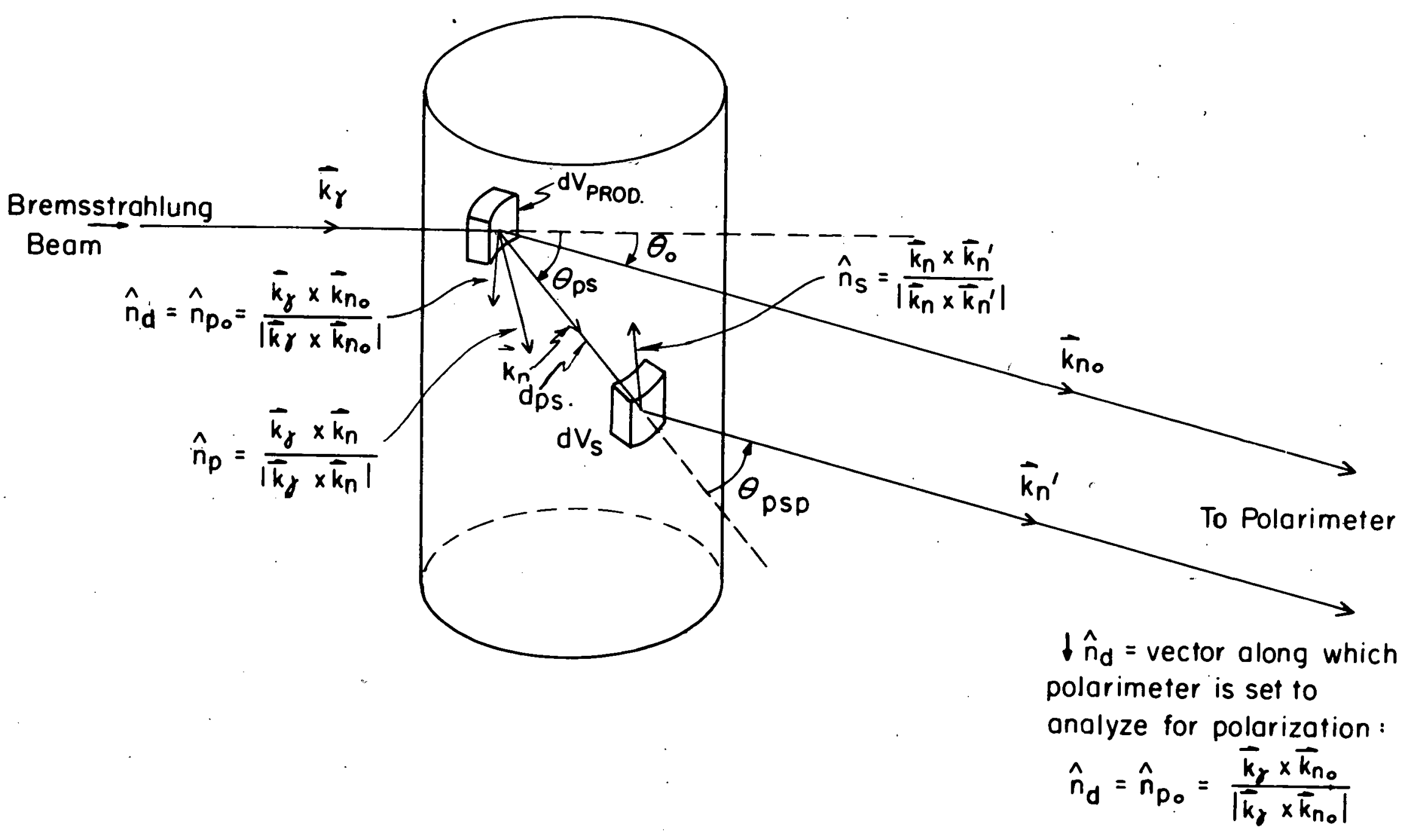

Geometry for Scattering within the Photoneutron Target

Fig. VI-14 
$\mathrm{N}_{\text {td }}(\mathrm{i})$ the equation

$$
N_{t d}(i)=\sum e^{-\left(a_{\gamma} d_{B}\right)^{2}} N_{\gamma}(i) e^{-d_{\gamma} / \lambda} \gamma n_{p r o d} d V_{\text {prod }} \frac{d \sigma}{d \Omega} \gamma n_{0}(\theta) e^{-d_{o u t} / \lambda_{n_{0}}}
$$

VI. 13

where

$N_{\gamma}(i)=$ the number of $\gamma$-rays $/ \mathrm{cm}^{2}$ incident on the center of the photoneutron target; $N_{\gamma}(i)$ includes all $\gamma^{\text {-rays }}$ which produce neutrons at $\theta_{0}$ in the energy interval $E_{\text {in }}(i)$.

$e^{-\left(a_{\gamma} d_{B}\right)^{2}}=$ a factor to account for the forward peaking of the bremsstrahlung beam. $\left(a_{\gamma}\right)^{-1}$ is the distance off the bremsstrahlung axis, at the target, where the bremsstrahlung intensity falls to $\frac{1}{e}$ of the central intensity. $d_{B}$ is the distance from the bremsstrahlung axis to the center of the production volume element $\mathrm{dV}_{\text {prod }} \cdot$

$\mathrm{e}^{-\mathrm{d}_{\gamma} /_{\lambda \gamma}}=$ the transmission of the bremsstrahlung by the target material. $\lambda_{y}$ is the mean free path in the target material for $\gamma$-rays of energy $E_{\gamma} ; d_{\gamma}$ is the distance through target material which the bremsstrahlung must pass to get to $\mathrm{dV}_{\text {prod }}$. $\mathrm{n}_{\text {prod }}=$ number of production nuclei $/ \mathrm{cm}^{3}$; this refers to the target nucleus of interest, since other production nuclei have already been subtracted as background. $\mathrm{dV}_{\text {prod }}=$ volume element in which production takes place.

$\frac{d \sigma}{d \Omega} \gamma_{0} n_{0}\left(\theta_{0}\right)=$ laboratory photoneutron cross section, for $E_{\gamma}$, with the neutron coming out at a laboratory angle of $\theta_{0}$. 
$\mathrm{e}^{-\mathrm{d} \text { out } / \lambda_{n_{0}}}=$ transmission factor for the outgoing neutron. $\lambda_{n_{0}}$ is the mean free path of the outgoing neutron; $\mathrm{d}_{\text {out }}$ is the distance from $d V_{p r o d}$ to the edge of the target, which the neutron Iravels.

The sum.in eq. VI.13 goes over all volume elements $\mathrm{dV}_{\text {prod. }}$

For $N_{\text {tind }}(i)$ the equation is

$$
\begin{aligned}
& N_{t} \text { ind }(i)=\Sigma e^{\prime}-\left(a_{\gamma} d_{B}\right)^{2} N_{\gamma}(j) e^{-d_{\gamma / \lambda_{\gamma}} n_{p r o d} d V_{p r o d} \frac{d \sigma}{d \Omega \gamma} n\left(\theta_{p s}\right) \frac{e^{-d_{p s} / \lambda}}{r^{2}} p s} \\
& \dot{x} n_{s} d V_{s} \frac{d_{\theta g} n}{d_{\Omega}}\left(\theta_{p s p}\right) e^{-d_{o u t} / \lambda n^{\prime}}\left[\frac{1}{\left[1+P_{s}\left(\theta_{p s p}\right) P_{n}\left(\theta_{p s}\right)\left(\hat{n}_{p} \cdot \hat{n}_{s}\right)\right]}\right]
\end{aligned}
$$

\section{14}

where the extra terms are

$$
\begin{aligned}
& { }^{\theta} \text { ps }=\text { the photoneutron production angle. } \\
& e^{-d} \text { ps/ } n=\text { transmission factor for the photoneutron going from } \\
& d V_{\text {prod }} \text {, the production volume, to } \mathrm{dV}_{\mathrm{s}} \text {, the scattering } \\
& \text { volume. } d_{p s} \text { is the distance through target material } \\
& \text { from } d V_{\text {prod }} \text { to } \mathrm{dV}_{\mathrm{s}} \text {, which equals } \mathrm{r}_{\mathrm{ps}} \text { for'the solid } \\
& \text { cylinder, but may be less than } r_{p s} \text { for the spherical } \\
& \text { she } 11 \text {. } \\
& r_{\text {ps }}=\text { the distance from } d V_{\text {prod }} \text { to } d V_{S} \text {. } \\
& \mathrm{n}_{\mathrm{S}} \quad=\text { the number of scattering nuclei/ } \mathrm{cm}^{3} \text {. } \\
& \mathrm{dV}_{\mathrm{S}}=\text { the volume element in which the neutron scattering } \\
& \text { takes place. } \\
& \frac{d \sigma}{d \Omega} n\left(\theta_{\text {psp }}\right)=\text { the neutron scattering differential cross section for } \\
& \text { nuclei corresponding to } \mathrm{n}_{\mathrm{s}} ; \theta_{\text {psp }} \text { is the scattering } \\
& \text { angle, } d V_{\text {prod }} \text { to } \mathrm{dV}_{\mathrm{S}} \text { to the polarimeter. }
\end{aligned}
$$


$e^{-d_{\text {out }} / \lambda^{\prime}} n=$ transmission factor for the outgoing neutron, of energy $E_{n^{\prime}}=E_{i n}(i)$.

The sum in eq. VI.14 goes over all $N(j), d V_{\text {prod }}$ and $d V_{s}$, with the prime indicating that the outgoing neutron $E_{n}$ ', must be in the $i$ 'th neutron energy interval.

The polarization is obtained from

$$
\begin{aligned}
& P_{t_{\text {ind }}}(i)=\frac{1}{N_{\text {tind }}(i)} \Sigma^{\prime} e^{-\left(a \gamma d_{B}\right)^{2}} N_{\gamma}(j) e^{-d \gamma} / \lambda_{\gamma} n_{\text {prod }} d V_{\text {prod }}
\end{aligned}
$$

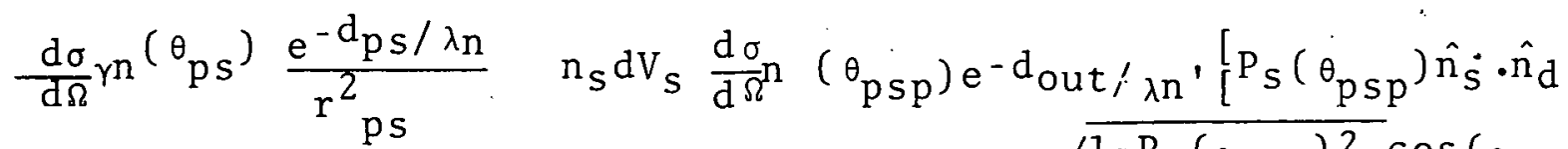

$$
\begin{aligned}
& +P_{n}\left(\theta_{p s p}\right)\left[D_{s}\left(\theta_{p s p}\right)\left(\hat{n}_{s} \cdot \hat{n}_{d}\right)\left(\hat{n}_{p} \cdot \hat{n}_{s}\right)+\frac{\sqrt{1-P_{s}\left(\theta_{p s p}\right)^{2}} \cos \left(\theta_{p s p}\right)}{1-\cos ^{2} \theta_{p s p}}\right.
\end{aligned}
$$

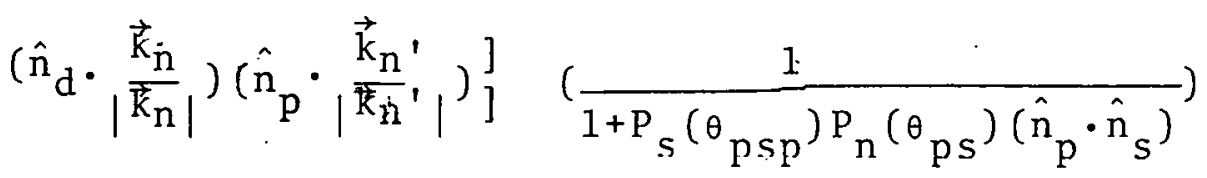

VI .15

where the extra terms are

$$
\begin{aligned}
& \mathrm{P}_{\mathrm{n}}\left({ }_{\mathrm{ps}}\right) \quad=\text { the production polarization of neutrons, at angle. } \\
& { }^{\theta} \text { ps, and for } \gamma \text {-rays of energy } E_{\gamma}(j) \text { giving neutrons } \\
& \text { of energy } E_{n} \text {. } \\
& \mathrm{P}_{\mathrm{s}}\left({ }_{\mathrm{psp}}\right) \quad=\text { the polarizing power of the scattering nuclei, at } \\
& \text { angle }{ }_{\mathrm{psp}} \text {, for neutrons of energy } \mathrm{E}_{\mathrm{n}} \text {. } \\
& D_{s}\left(\theta_{p s p}\right) \quad=\text { the depolarization parameter of the scattering } \\
& \text { nuclei, at angle } \theta_{\text {psp }} \text {, for neutrons of energy } E_{n} \text {. } \\
& \hat{\mathrm{n}}_{\mathrm{d}}=\text { the direction along which the polarimeter is set } \\
& \text { to measure polarization. }
\end{aligned}
$$




$$
\begin{aligned}
& \hat{n}_{p}=\frac{\vec{k}_{\gamma} \times \vec{k}_{n}}{\left|\vec{k} \vec{k}_{\gamma} \times \vec{k}_{n}\right|} \\
& \hat{n}_{s}=\frac{\vec{k}_{n} \times \vec{k}_{n^{\prime}}}{\left|\vec{k}_{n} \times \vec{k}_{n}^{\prime}\right|}
\end{aligned}
$$

$\overrightarrow{\mathrm{k}}_{\mathrm{n}}, \overrightarrow{\mathrm{k}}_{\mathrm{n}}{ }^{\prime}=$ the neutron wave vectors, before and after the neutron scattering.

The polarization terms in eq. VI.15 are taken from Williams, An Introduction to Elementary Particles ${ }^{(57)}, \mathrm{p} 187$.

The sums for $\mathrm{N}_{t d}(\mathrm{i}), \mathrm{N}_{\text {tind }}(\mathrm{i})$, and $\mathrm{P}_{\text {tind }}(\mathrm{i})$ were calculated using the uncorrected measurements for $\mathrm{P}_{\mathrm{n}}\left(\theta_{\mathrm{ps}}\right)$. The angular distribution of $P_{n}\left(\theta_{p s}\right)$ was assumed to be of the form

$$
\mathrm{P}_{\mathrm{n}}\left(\theta_{\mathrm{ps}}\right)=\mathrm{P}_{1} \sin \left(\theta_{\mathrm{ps}}\right)+\mathrm{P}_{2} \sin \left(2 \theta_{\mathrm{ps}}\right)
$$

and where possible, fitted by the uncorrected experiments at 45 and 90 degrees. The photoneutron cross sections were obtained from the measurements of Verbinski and Courtney (26) for $0^{16}$ and $\mathrm{C}^{12}$. $\mathrm{Ca}^{40}$ had its total photoneutron cross section fitted to an angular distribution of $\left(1+3 / 2 \sin ^{2} \theta_{\mathrm{ps}}\right)$. This is the angular distribution for $0^{16}$ and $\mathrm{C}^{12}$, and was chosen arbitrarily. Since the target scattering corrections for $\mathrm{Ca}^{40}$ are insignificant compared with other errors, this assumption is not critical. The photoneutron cross section angular distribution for $\mathrm{Pb}^{208}$ was obtained from the results of G. Mutchler (58).

The photon spectra used in the calculations were thick target bremsstrahlung spectra attenuated by the $8^{\prime \prime}$ of water of the beam stopper. The photons were distributed over the target in a Gaussian shape, with the theoretical angular width obtained 
from ref.(65)

The polarization parameters of the neutron scattering, $\mathrm{P}_{\mathrm{s}}\left(\theta_{\mathrm{psp}}\right)$ and $\mathrm{D}_{\mathrm{s}}\left(\theta_{\mathrm{psp}}\right)$, were generally set to 0 and 1 respectively, for lack of information. $D_{s}\left(\theta_{p s p}\right)$ should be 1 for spin zero nuclei. For $\mathrm{C}^{12}$ neutron scattering the phase shifts are available $(59,60)$ to calculate $\mathrm{P}_{\mathrm{S}}\left(\theta_{\mathrm{psp}}\right)$ and these calculated values were used in eqs. VI.14 and VI.15.

For targets composed of a number of elements, e.g. oxalic acid, with oxygen, carbon and hydrogen, the sums of eqs. VI.14 and VI.15 were carried out for all types of scattering nuclei. The loss of energy in elastic neutron scattering was always calculated, unlike the polarimeter and tunnel scattering calculations. This was necessary since scattering from hydrogen in the oxalic acid and water targets produces large losses in neutron energy, so $E_{n}{ }^{\prime}$ can be very much less than $E_{n}$.

The error in the target scattering corrections is taken to be $30 \%$ of their magnitude. As given in chapter IV, this means that (eq. IV.39)

$$
\begin{aligned}
& \Delta P_{t s}=0.3 P_{t s} \\
& \Delta k_{t s}=0.3 k_{t s}
\end{aligned}
$$$$
\text { VI. } 16
$$

With eq. 'VI.16, the target scattering correction calculations are complete, to be used as shown in Chapter IV. 
CHAPTER VII

\section{DISCUSSION OF THE RESULTS AND CONCLUSIONS}

\section{A. General Considerations on Photonuclear Reactions}

When $\left(\frac{R}{\lambda}\right)<<1$ the interaction of a photon beam and a nucleus may be conveniently described in terms of the interaction of photons in a specific angular momentum state: Thus photoabsorption is described as El $\left(\mathrm{L}^{\pi}=1^{-}\right), \mathrm{M} 1\left(\mathrm{~L}^{\pi}=1^{+}\right), \mathrm{E} 2\left(\mathrm{~L}^{\pi}=2^{+}\right)$, etc., where $L$ th is the photon angular momentum absorbed by the nucleus and $\pi= \pm$ indicates whether the nuclear parity has changed. We can also define $\mathrm{g}=\mathrm{L}, \pi=(-1)^{\mathrm{g}+1-\mathrm{p}}$ where $\mathrm{p}=0$ for magnetic(M) transitions and $p=1$ for electric (E) transitions, and where $(g, p)$ is the notation used by Baldin et a1 in Kinematics of Nuclear Reactions. $(66)$

In a photonuclear reaction a nucleus with spin-parity $\left(j_{\Pi}, \pi_{I}^{\prime}\right)$ (initial nuclear angular momentum $=j_{\Pi} \mathrm{h}$ ) absorbs a photon $(\mathrm{g}, \mathrm{p})$. to give an intermediate state $(J, \pi)$, which then emits a particle with spin-parity $\left(j_{1}, \pi_{1}^{i}\right)\left(j_{1}{ }^{{ }^{\prime}}=1 / 2^{+}\right.$for a neutron $)$and orbital angular momentum $\ell$, leaving a final nucleus with spin-parity $\left(j_{2}, \pi_{2}^{\prime}\right) \cdot\left(j_{2}{ }^{\pi} 2^{\prime}=1 / 2^{-}\right.$for the $\left.0^{16} \mathrm{~g} . \mathrm{s}.\right)$. The final state spin is $s$, where $\vec{s}=\vec{j}_{1}+\vec{j}_{2}$ and the total angular momentum is $J$, where $\vec{J}=\vec{s}+\vec{\ell}$ (angular momenta are expressed in units of $t$ ). If we let $\theta$ be the angle between the incoming photon $\left(\vec{k}_{\gamma}\right)$ and the outgoing neutron $\left(\vec{k}_{n}\right)$, then from conservation of angular momentum and the properties of the electromagnetic field we obtain for the cross section and polarization for this reaction 


$$
\begin{aligned}
\frac{\mathrm{d} \sigma}{\mathrm{d} \Omega}= & \frac{\pi^{2}}{2\left(2 j_{I I}+1\right)} \sum \frac{(-1)^{s^{\prime}-j_{\Pi^{-1}}}}{4} \operatorname{Re}\left\{\left(\ell_{1}^{\prime} s^{\prime} \alpha^{\prime}\left|R_{\Pi_{1}}^{J_{1}}\right| p_{1} g_{1} \alpha\right)\right. \\
& \left.\times\left(\ell_{2}^{\prime} s^{\prime} \alpha^{\prime}\left|R_{\Pi_{2}}^{J} 2\right| p_{2} g_{2} \alpha\right)\right\}(-1)^{p_{1}+p_{2}}(i)-\ell_{2}^{\prime}+\ell_{1}^{\prime}-L \\
& \times Z\left(\ell_{1}^{j} J_{2} \ell_{2}^{\prime} J_{2} ; s^{\prime} L\right) Z_{\gamma}\left(g_{1} j_{1} g_{2} J_{2} ; j_{I I} L\right) P_{L}(\cos \theta)
\end{aligned}
$$

VII. I

and

$$
\begin{aligned}
& \frac{d \vec{P}}{d \Omega}=\frac{\vec{k}_{\gamma} \times \vec{k}_{n}}{\left|\vec{k}_{\gamma} \times \vec{k}_{n}\right|} \frac{\pi^{2}}{4} \frac{\left\{2 j_{1}\left(j_{1}+1\right)\left(2 j_{1}+1\right)\right\}^{1 / 2}}{2\left(2 j_{I I}+1\right)} \\
& \times\left[\operatorname{Re}\left\{i\left(\ell_{1}^{\prime} s_{1} \alpha^{\prime}\left|R_{\Pi_{1}}^{J}\right| p_{1} g_{1} \alpha\right)^{*}\left(\ell_{2}^{\prime} s_{2}^{\prime} \alpha^{\prime}\left|R_{\Pi_{2}}^{J} 2\right| p_{2} g_{2} \alpha\right)\right\}\right. \\
& \times(-I)^{j_{2}-j_{1}-j_{I I}+J_{2}+s i_{2}+l_{2}^{\prime}-1} z_{\gamma}\left(g_{1} J_{1} g_{2} J_{2} ; j_{I I} L\right) \\
& \times(-1)^{p_{1}+p_{2}} w\left(j_{1} s_{1} j_{1} s_{2}^{\prime} ; j_{2} 1\right)\left\{\left(2 J_{1}+1\right)\left(2 \ell_{1}+1\right)\left(2 s_{1}+1\right)\right. \\
& \left.\times\left(2 J_{2}+1\right)\left(2 \ell_{2}^{\prime}+1\right)\left(2 s_{2}^{\prime}+1\right)\right\}^{1 / 2}\left(\ell_{1}^{\prime} 0 \ell_{2}^{\prime} 0 \mid L 0\right) \\
& \times X\left(J_{1} \ell_{1}^{\prime} s_{1}^{\prime} ; J_{2} \ell_{2}^{\prime} s_{2}^{\prime} ; L L 1\right) \overline{\mathrm{P}_{L}^{1}}(\cos \theta)
\end{aligned}
$$

VI I . 2

where the sums are over $J_{1}, J_{2}, g_{1}, g_{2}, p_{1}, p_{2}, \ell_{1}^{\prime}, \ell_{2}^{\prime}, s_{1}^{\prime}$, $s_{2}^{\prime}, s^{\prime}$, and $L$. These formulas are obtained from Baldin et a1 ${ }^{(66)}$, pp 185-6. For definitions of the $z, z_{\gamma}$, and $X$ coefficients see Baldin et al. The matrix elements $\left(\ell^{\prime} s^{\prime} \alpha^{\prime}\left|R_{\Pi}^{J}\right| p g \alpha\right)$ depend on the purely internal nuclear quaritum numbers $\alpha^{\prime}$ and $\alpha$, and on the coupling of these states with the E-M field via the intermediate state $(J, \pi)$ to the final state $\left(l^{\prime}, s^{\prime}\right)$. The matrix elements also depend on $j_{1}$, the intrinsic spin of the outgoing particle, and $j_{2}$, the spin of the final nulceus, the channel spin $s$ being the vector sum of these, $\vec{s}=\vec{j}_{1}+\vec{j}_{2}$. 
The polarization defined in eq. VII.2 is the expectation value of the spin operator for the outgoing nucleon, and must be modified to give the polarization of the neutron as defined earlier in this thesis. For photoneutrons the polarization we have been discussing is defined by

$$
\vec{P}_{n}=\frac{1}{j_{1}} \frac{\left(\frac{d \vec{P}}{d \Omega}\right)}{\left(\frac{d \sigma}{d \Omega}\right)}
$$

VII. 3

where $j_{1}=1 / 2$. This is the relative polarization as defined by Baldin et a1. The differential cross section of eq. VII.1 and the polarization of eq. VII.3 are the quantities to be compared with experimental measurements. The next three sections will discuss the application of eqs. VII.1, VII.2 and VII.3 to the $0^{16}(\gamma, n), C^{12}(\gamma, n)$ and $\mathrm{Pb}^{208}(\gamma, n)$ results, using the polarizations presented in this thesis and cross section measurements of other experimenters.

B. Interpretation of the $0^{16}(\gamma, n)$ Results

The $0^{16}\left(\gamma, n_{0}\right) 0^{15}$ reaction has an initial nuclear spinparity of $0^{+}$for $0^{16}$ and a final nuclear spin of $1 / 2^{-}$for $0^{15}$. The intermediate state-spin-parity is completely determined by the multipole, i.e. $(J, \pi)$ is determined by $(g, p)$, and thus the notation of eqs. VII.I and VII.2 can be simplified. The results of eqs. VII.l and VII.3 for pure E1 transitions are

$$
\frac{d \sigma}{d \Omega}=\frac{\pi^{2}}{8}\left\{3.0\left[A_{S}^{2}+A_{d}^{2}\right]+\left[4.24 A_{S} A_{d} \cos \Delta-1.5 A_{d}^{2}\right]\left(\frac{3 \cos 2 \theta+1}{4}\right)\right\}
$$




$$
\overrightarrow{\mathrm{P}}_{n}=\left(\frac{\overrightarrow{\mathrm{k}}_{\gamma} \times \overrightarrow{\mathrm{k}}_{n}}{\left|\overrightarrow{\mathrm{k}}_{\gamma} \times \overrightarrow{\mathrm{k}}_{n}\right|}\right) \frac{\left[-3.286 \mathrm{~A}_{s} \mathrm{~A}_{\mathrm{d}} \sin \Delta\right](\sqrt{15 / 16} \sin 2 \theta)}{\left\{3\left[\mathrm{~A}_{\mathrm{s}}^{2}+\mathrm{A}_{d}^{2}\right]+\left[4.24 \mathrm{~A}_{s} A_{d} \cos \Delta-1.5 A_{d}^{2}\right]\left(\frac{3 \cos 2 \theta+1}{4}\right)\right\}}
$$

VII. 5

where we have set $\left(0,1, \alpha^{\prime}\left|R_{-}^{1}\right| 1,1, \alpha\right)=A_{S} e^{i \delta},\left(2,1, \alpha^{\prime}\left|R_{.}^{1}\right| 1,1, \alpha\right)$ $=\mathrm{A}_{\mathrm{d}} \mathrm{e}^{\mathrm{i} \delta} \mathrm{d}$ and $\Delta=\delta_{\mathrm{d}^{-}} \delta_{\mathrm{s}}$. The general results of eqs. VII.1 and VII.2 with all E1, M1 and E2 terms included are given in appendix IV for reference. Using a neutron time-of-flight method with bremsstrahlung from $34 \mathrm{MeV}$ electrons Verbinski and Courtney $(26)$ measured the photoneutron spectra at $55^{\circ}$, $93^{\circ}$ and $141^{\circ}$ to the photon beam. The $6.2 \mathrm{MeV}$ neutron peak from the $22.1 \mathrm{MeV}$ excitation of $0^{16}$ was normalized to a $\left(1+\frac{3}{2} \sin ^{2} \theta\right)$ angular distribution, and on this basis they found that all neutrons in the energy range 2 to $9 \mathrm{MeV}$ also have a $\left(1+\frac{3}{2} \sin ^{2} \theta\right)$ angular distribution. Their assumption of a $\left(1+\frac{3}{2} \sin ^{2} \theta\right)$ distribution for the $6.2 \mathrm{MeV}$ neutron peak was motivated by the results of Earle, Tanner and Thomas (29) for. the $\mathrm{N}^{15}\left(\mathrm{p}, \gamma_{0}\right) \mathrm{O}^{16}$ reaction, and by the $\mathrm{O}^{16}\left(\mathrm{e}, \mathrm{e}^{\prime} \mathrm{p}\right)$ measurements of Dodge and Barber (33).

The angular distribution results thus give

$$
\begin{aligned}
\frac{\mathrm{d} \sigma}{\mathrm{d} \Omega} & =a\left(1+\frac{3}{2} \sin ^{2} \theta\right) \\
& =a\left[2-\left(\frac{3 \cos 2 \theta+1}{4}\right)\right] \\
& =a\left[2-P_{2}(\cos \theta)\right]
\end{aligned}
$$

which from eq. VII.4 gives

$$
\frac{3\left(A_{s}^{2}+A_{d}^{2}\right)}{\left(4.24 A_{s} A_{d} \cos \Delta-1.5 A_{d}^{2}\right)}=\frac{2}{(-1)}
$$


The polarization meaşurements at $45^{\circ}$ give(see fig. VII-1) $P_{n}=+0.50 \pm 0.05$ along $\frac{\vec{k}_{\gamma} \times \vec{k}_{n}}{\left|\vec{k}_{\gamma} \times \vec{k}_{n}\right|}$ for $E_{n} \simeq 5$ to $9 \mathrm{MeV}$. From eq. VII. 5 this gives

$$
\begin{gathered}
+(0.50 \pm 0.05)=\frac{3.18 A_{s} A_{d}(-\sin \Delta)}{3\left(A_{S}^{2}+A_{d}^{2}\right)+\left(4.24 A_{S} A_{d} \cos \Delta-1.5 A_{d}^{2}\right)(1 / 4)} \\
\text { VII. } 8
\end{gathered}
$$

Eqs. VII.7 and VII.8 can both be solved to give the two solutions

$$
\begin{array}{r}
A_{S}=\left(1.39 \begin{array}{r}
-0.26 \\
+0.21
\end{array}\right) A_{d}, \Delta=\delta_{d}-\delta_{s}=-\left(118 \begin{array}{r}
+4 \\
-6
\end{array}\right)^{\circ} \\
\text { VII. } 9
\end{array}
$$

and

$$
A_{S}=\left(0.55 \begin{array}{l}
+0.15 \\
-0.10
\end{array}\right) A_{d}, \Delta=\delta_{d}-\delta_{S}=-\left(\begin{array}{rr}
101 & +3 \\
-2
\end{array}\right)^{\circ}
$$

VI I. 10

where only the errors in the polarization have been included.

The measured polarization at $45^{\circ}$ is close to the maximum possible value for El photon absorption. Assuming a $\left(1+\frac{3}{2} \sin ^{2} \theta\right)$ angular distribution for $\frac{d \sigma}{d \Omega}$ the maximum value of $\mathrm{P}_{\mathrm{n}}\left(45^{\circ}\right)$ in eq VII.8 is $\mathrm{P}_{\mathrm{n}} \approx+0.57$, for $\mathrm{A}_{\mathrm{s}}=\mathrm{A}_{\mathrm{d}}$ and $\Delta=\delta \mathrm{d}^{-\delta} \mathrm{s}$ $=-1.11^{\circ}$. If no restrictions are applied to $\frac{\mathrm{d} \sigma}{\mathrm{d} \Omega}$ then the maximum is $\mathrm{P}_{\mathrm{n}}=+0.577$ for $\mathrm{A}_{\mathrm{s}}=0.936 \mathrm{~A}_{\mathrm{d}}$ and $\Delta=-100.9^{\circ}$.

Eq. VII.7 alone can be solved by setting $A_{S}=0$, which is effectively what Verbinski and Courtney ${ }^{(26)}$ concluded when they assumed pure d-wave neutron emission. This conclusion is not unique, however, as a large $s$-wave component can be present and give the same angular distribution if $\cos \Delta=$ $-0.354\left(A_{s} / A_{d}\right)$. The maximum ratio of $\left(A_{s} / A_{d}\right)$ consistent with 
the angular distribution is thus 2.82 when $\cos \Delta=-1$, which is largely s-wave neutron emission. Similarly, the conclusions of Earle et al ${ }^{(29)}$ that the reaction $N^{15}\left(p, \gamma_{0}\right)$ goes primarily by $d$-wave proton absorption is questionable, as their angular distribution cannot yield both the $s$ and $d$ wave amplitude ratio and phase difference.

Calculations of the polarization of $0^{16}$ photoneutrons at $45^{\circ}$ have been made by Weiss ${ }^{(15)}$ and Buck and Hill(16), using sheli model states in a finite well. Fig. VII-I shows the results of their calculations compared with some of the experimental results for $0^{16}$ with 24,30 , and $38 \mathrm{MeV}$ bremsstrahlung. For reference fig. VII-1 also shows the $90^{\circ}$ photoneutron cross section of Verbinski and Courtney(26). The experimental $45^{\circ}$ neutron polarization for $0^{16}\left(\gamma, n_{0}\right)$ displays a large positive polarization of about +0.50 , with a slight peaking over the giant resonance of 21 to $26 \mathrm{MeV}$ photon energy. For the giant resonance Weiss, and Buck and Hill both calculate a large positive polarization. Weiss. calculates a sharp drop in the polarization on each side of the giant resonance, while Buck and Hill give a slow decrease for energies above the giant resonance and a sharp drop below the resonance which occurs about $2 \mathrm{MeV}$ lower than that of Weiss. The experimental results show substantial agreement with the calculations of Buck and Hill, and do not show the sharp changes in polarization calculated by Weiss. Raphael and Überall ${ }^{(67)}$ have also calculated the polarization at $45^{\circ}$, but only for the $22.3 \mathrm{MeV}$ excitation for which they obtain $P_{n}=+0.39$. 


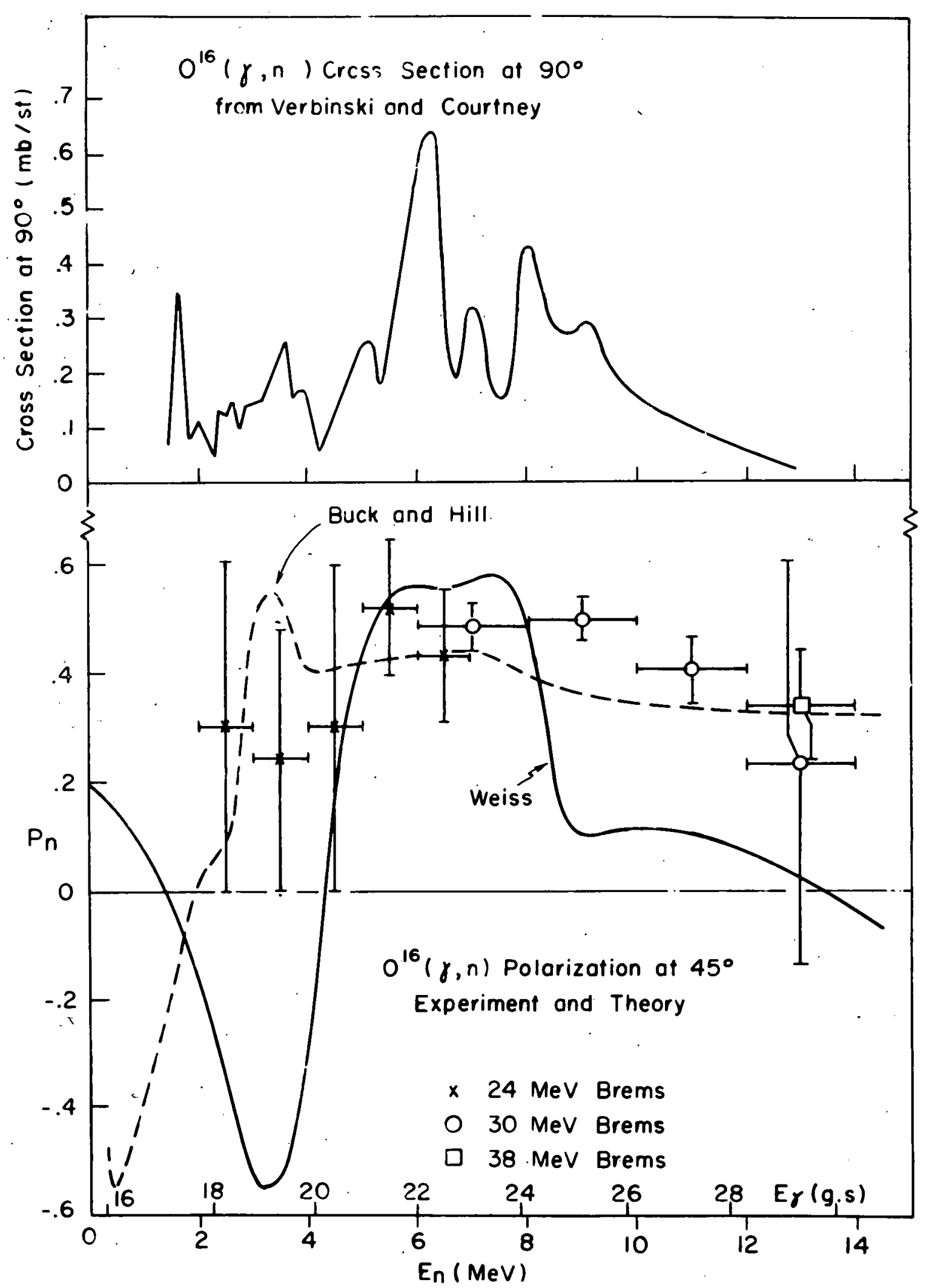

Experimental and Theoretical Polarizations of $0^{16}(\gamma, n)$ Neutrons at $45^{\circ}$

Fig. 'VI I - I 
The calculations of Weiss and of Buck and Hill show the same very general features, a large positive polarization for the giant resonance which decreases for energies above and below the resonance. The better agreement of experiment with the calculations of Buck and Hill probably reflects the more general average potential and particle-hole interaction which they used. Weiss used a square well and a particlehole interaction which was a $\delta$-function at the nuclear surface, while Buck and Hill used a Saxon-Woods shape and a particle-hole interaction which was a $\delta$-function in their relative coordinates and thus not confined to the nuclear surface.

Fig. VII-2 shows all the experimental $0^{16}$ polarization results at $45^{\circ}$ for 24,30 and $38 \mathrm{MeV}$ bremsstrahlung and a plot of the results at $90^{\circ}$ for $30 \mathrm{MeV}$ bremsstrahlung. The polarization measurements using $3.0 \mathrm{MeV}$ bremsstrahlung should give the polarization of the $\mathrm{g} . \mathrm{s}$. decay of the $0^{16} \mathrm{giant}$ resonance ( 21 to $25 \mathrm{MeV}$ photon energy) with little contamination from non-g.s. transitions. Caldwell et al (22) have measured the g.s. neutron emission cross section as well as the cross section for the $6.17 \mathrm{MeV} 3 / 2^{-}$state in $0^{15}$. The sum of the cross sections for the $5.17 \mathrm{MeV} 1 / 2^{+}$and $5.24 \mathrm{MeV} 5 / 2^{+}$states of $0^{15}$ was found to be negligible, about 0.5 barns for 21 to $21.5 \mathrm{MeV}$ photons ( $\simeq 1 / 2 \mathrm{MeV}$ neutrons) and zero elsewhere. Thus for $30 \mathrm{MeV}$ bremsstrahlung only the transitions to the $6.17 \mathrm{MeV}$ state of $0^{15}$ are important when calculating the purity of neutrons from g.s. transitions of the $0^{16}$ giant resonance. The $8.1 \mathrm{MeV}$ neutrons come from the peak at 24.3 


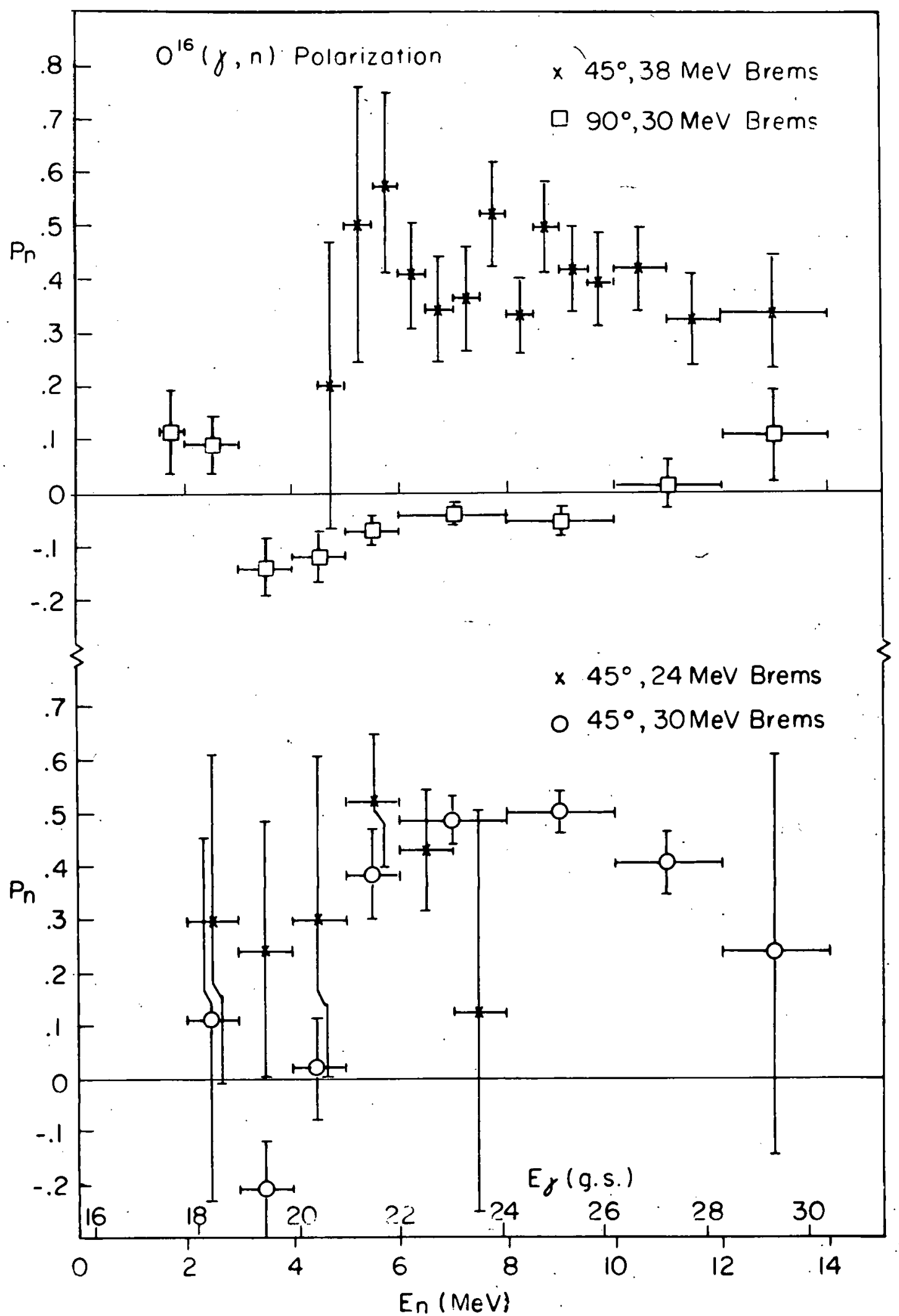

Comparison of Experimental $0^{16}(\gamma, n)$ Neutron Polarizations at $45^{\circ}$ and $90^{\circ}$

Fig. VII-2 
MeV excitation and are essentially pure, since transitions to the $6.17 \mathrm{MeV}$ level require $30.5 \mathrm{MeV}$ photons if they are to emit an $8.1 \mathrm{MeV}$ neutron. The $6.2 \mathrm{MeV}$ neutrons from the $22.3 \mathrm{MeV}$ excitation may have a $20 \%$ contamination from transitions to the $6.17 \mathrm{MeV}$ state in $0^{15}$. The $6.2 \mathrm{MeV}$ neutrons from the latter transition arise from $28.5 \mathrm{MeV}$ photons, which are about half as intense as the $22.3 \mathrm{MeV}$ photons and have about half the cross section. This conclusion assumes that both types of neutrons(g.s. and excited state)have the same angular distribution, as the only cross section comparison is between the total cross sections of Caldwell et al.

The $45^{\circ}$ polarization measurements with $38 \mathrm{MeV}$ bremsstrahlung exhibit a slight structure over the giant resonance, which may be due to a contamination from non-g.s. transition neutrons. The partial results for $30 \mathrm{MeV}$ shown in figs. V-3 and V-4(pp 122-3) exhibit less structure, though the energy scale is broken into only 1 MeV widths as compared with $1 / 2 \mathrm{MeV}$ widths for the $38 \mathrm{MeV}$ results. These data were taken with the stainless steel gas chamber, which had sufficient resolution to separate the $6.2 \mathrm{MeV}$ and $8.1 \mathrm{MeV}$ neutron groups from the $0^{16}$ excitations at $22.3 \mathrm{MeV}$ and 24.3 $\mathrm{MeV}$, as is shown by the measured neutron spectra in fig. IV-1b ( 96$)$.

The polarization of neutrons emitted by transitions to the $6.17 \mathrm{MeV}$ excited state of $0^{15}$ may be obtained by comparing the measured $45^{\circ}$ polarizations for 24 and $30 \mathrm{MeV}$ bremsstrahlung. We confine our considerations to the measurements for neutrons of 3-4 MeV, which arise from $19.4 \mathrm{MeV}$ photons for g.s. 
transitions, and from $25.6 \mathrm{MeV}$ photons for transitions to the $6.17 \mathrm{MeV}$ excited state. The cross. section measurements of Caldwe 11 et al (22) show that these are the only transitions that need be considered for the bremsstrahlung energies in question. The polarization measurements for $24 \mathrm{MeV}$ bremsstrahlung thus yield $\mathrm{P}_{\mathrm{gs}}(19.4)=+0.24 \pm 0.24$ for the $\mathrm{g} . \mathrm{s}$. neutron polarization. Using the measured cross sections of Verbinski and Courtney (26) and of Caldwell et al (22), and assuming a $\left(1+\frac{3}{2} \sin ^{2} \theta\right)$ angular distribution for both, we can obtain

$$
\left.\mathrm{P}_{\mathrm{ex}}(25.6)\right|_{45^{\circ}}=-0.35 \pm 0.20 \quad \text { VII.11 }
$$

The calculations to obtain eq. VII.11 are given in appendix $V$, where it is also stated that the error is derived only from the errors in the polarization measurements and thus may be too small. Weiss (15) has calculated the $6.17 \mathrm{MeV}$ excited state transition neutron polarization at $45^{\circ}$ to be about -0.05 for a photon energy of $25.6 \mathrm{MeV}$. This is not considered to be in disagreement with the results VII.11 because of the crude estimates used to obtain eq. VII.11.

For the two dominant peaks of the giant resonance the $90^{\circ}$ polarization is approximately $-0.05 \pm 0.02$. If this arises from an E2 interference, as is suggested by the $\left(e, e^{\prime} p\right)$ results of Dodge and Barber (33), then an estimate for the amplitude of the interfering waves may be made. The E2 transition brings in two amplitudes and phases, $A_{p} e^{i \delta} p=\left(1,1, \alpha^{\prime}\left|R_{+}^{2}\right| 1,2, \alpha\right)$ and $A_{f} e^{i \delta} f=\left(3,1, \alpha^{\prime}\left|R_{+}^{2}\right| 1,2, \alpha\right)$. If we assume that the El s-and d-wave amplitudes are equal, 
and use the angular distribution results of Verbinski and Courtney ${ }^{(26)}$, then we can obtain

$$
\begin{aligned}
P_{n}\left(90^{\circ}\right)= & -0.32\left(\frac{A_{p}}{A}\right) \sin \left(\delta_{s}-\delta_{p}\right)-0.26\left(\frac{A_{f}}{A}\right) \sin \left(\delta_{s}-\delta_{f}\right) \\
& +0.45\left(\frac{A_{p}}{A}\right) \sin \left(\delta_{d}-\delta_{p}\right)+0.37\left(\frac{A_{f}}{A}\right) \sin \left(\delta_{d}-\delta_{f}\right)
\end{aligned}
$$

$$
\text { VII. } 12
$$

where $A_{S}=A_{d}=A$. In deriving eq. VII.12 interference effects on the cross section have been neglected. The minimum amplitude solution(for E2 absorption)of eq. VII.12 arises when $A_{f}=0$ and $\delta_{p}=\delta_{s}$. Since $\delta_{s} \simeq \delta_{d}+110^{\circ}$ from eqs. VII.9 and VII.10, and $P_{n}\left(90^{\circ}\right) \simeq-0.05$, this yields

$$
A_{p} \simeq 0.1 A
$$

Thus the $90^{\circ}$ polarization results require the amplitude of the interfering wave from the E2 state to be at least $10 \%$ of the El s-and d-wave amplitude. This result is by no means exact, as eq. VII.21 has two amplitude ratios and two relative phases unspecified, and can be solved in many other ways. However, the angular distribtuion results for both $0^{16}(\gamma, n)(26)$ and $0^{16}\left(e, e^{\prime} p\right)^{(33)}$ indicate that the E2 amplitude is small, and thus the neutron waves from E2 excitation probably have only $10-20 \%$ of the amplitude of the El neutron waves.

C. Interpretation of the $\mathrm{C}^{12}(\gamma, \mathrm{n})$ Results

The $\mathrm{C}^{12}\left(\gamma, \mathrm{n}_{\mathrm{o}}\right)$ reaction is a transition from tne $0^{+} \mathrm{g} \cdot \mathrm{s}$. of $\mathrm{C}^{12}$ to the $3 / 2^{-} \mathrm{g} . \mathrm{s}$. of $\mathrm{C}^{11}$. The results of eqs. VII.1 and VII.2 for pure El excitations are, using a simplified notation 


$$
\begin{gathered}
\frac{\mathrm{d} \sigma}{\mathrm{d} \Omega}=\frac{\pi^{2}}{8}\left\{3\left(\mathrm{~A}_{\mathrm{s}}^{2}+\mathrm{A}_{\mathrm{d} 1}^{2}+\mathrm{A}_{\mathrm{d} 2}^{2}\right)+\left[4.24 \mathrm{~A}_{\mathrm{s}} \mathrm{A}_{\mathrm{d} 1} \cos \left(\delta_{\mathrm{d} 1}-\delta_{\mathrm{s}}\right)\right.\right. \\
\left.\left.-1.5 \mathrm{~A}_{\mathrm{d} 1}^{2}+1.5 \mathrm{~A}_{\mathrm{d} 2}^{2}\right]\left(\frac{3 \cos 2 \theta+1}{4}\right)\right\} \quad \text { VII. } 14
\end{gathered}
$$

and

$$
\begin{aligned}
\overrightarrow{\mathrm{P}}_{\mathrm{n}}=\frac{1}{j_{1}} \frac{\left(\frac{\mathrm{d} \overrightarrow{\mathrm{d}}}{\mathrm{d} \Omega}\right)}{\left(\frac{\mathrm{d} \sigma}{\mathrm{d} \Omega}\right)}= & 2 \frac{\overrightarrow{\mathrm{k}}_{\gamma} \times \overrightarrow{\mathrm{k}}_{\mathrm{n}}}{\left|\overrightarrow{\mathrm{k}}_{\gamma} \times \overrightarrow{\mathrm{k}}_{\mathrm{n}}\right|}(0.217)\left(0.237 \mathrm{~A}_{\mathrm{s}} \mathrm{A}_{\mathrm{d} 1} \sin \left(\delta_{\mathrm{s}}-\delta_{\mathrm{d} 1}\right)\right. \\
& -0.237 \mathrm{~A}_{\mathrm{s}} \mathrm{A}_{\mathrm{d} 2} \sin \left(\delta_{\mathrm{s}}-\delta_{\mathrm{d} 2}\right)+0.335 \mathrm{~A}_{\mathrm{d} 1} \mathrm{~A}_{\mathrm{d} 2} \\
& \left.\times \sin \left(\delta_{\mathrm{d} 1}-\delta_{\mathrm{d} 2}\right)\right\}(-\sqrt{15 / 16} \sin 2 \theta) \\
& \times\left[\frac { 1 } { 2 } \left\{3\left(\mathrm{~A}_{\mathrm{s}}^{2}+\mathrm{A}_{\mathrm{d} 1}^{2}+\mathrm{A}_{\mathrm{d} 2}^{2}\right)+\left(4.24 \mathrm{~A}_{\mathrm{s}} \mathrm{A}_{\mathrm{d} 1} \cos \left(\delta_{\mathrm{d} 1}-\delta_{\mathrm{s}}\right)\right.\right.\right. \\
& \left.\left.\left.-1.5 \mathrm{~A}_{\mathrm{d} 1}^{2}+1.5 \mathrm{~A}_{\mathrm{d} 2}^{2}\right)\left(\frac{3 \cos 2 \theta+1}{4}\right)\right\}\right]
\end{aligned}
$$

VI I . 15

where $\left(0,1, \alpha^{\prime}\left|R_{-}^{1}\right| 1,1, \alpha\right)=A_{s} e^{i \delta} s,\left(2,1, \alpha^{\prime}\left|R_{-}^{1}\right| 1,1, \alpha\right)=$ $A_{\mathrm{d} 1} \mathrm{e}^{\mathrm{i \delta} \mathrm{d} l}$, and $\left(2,2, \alpha^{\prime}\left|\mathrm{R}_{-}^{1}\right| 1,1, \alpha\right)=\mathrm{A}_{\mathrm{d} 2} \mathrm{e}^{\mathrm{i} \delta} \mathrm{d} 2$. The general results of eqs. VII.1 and VII.2 for $C^{12}(\gamma, n)$ with all El, M1 and E2 terms included are given in appendix. IV for reference.

The angular dependence of $\frac{\mathrm{d} \sigma}{\mathrm{d} \Omega}$ as given by Verbinski and Courtney $(26)$ is $\left(1+\frac{3}{2} \sin ^{2} \theta\right)$. This conclusion is based on the $\mathrm{B}^{11}\left(\mathrm{p}, \gamma_{0}\right)$ angular distribution ${ }^{(38)}$, the $\mathrm{C}^{12}\left(\mathrm{e}, \mathrm{e}^{\prime} \mathrm{p}\right)$ proton angular distribution $(33)$, and the $\mathrm{C}^{12}(\gamma, n)$ neutron angular distribution integrated for excitations greater than $22 \mathrm{MeV}(36)$. The measurements of Verbinski and Courtney indicate that this angular distribtuion is valid for neutrons in the energy region 2 to $10 \mathrm{MeV}$. The experimental results for $\frac{\mathrm{d} \sigma}{\mathrm{d} \Omega}$ require 


$$
\frac{3\left(\mathrm{~A}_{\mathrm{s}}^{2}+\mathrm{A}_{\mathrm{d} 1}^{2}+\mathrm{A}_{\mathrm{d} 2}^{2}\right)}{4.24 \mathrm{~A}_{\mathrm{s}} \mathrm{A}_{\mathrm{d} 1} \cos \left(\delta_{\mathrm{dl}}-\delta_{\mathrm{s}}\right)-1.5 \mathrm{~A}_{\mathrm{dl}}^{2}+1.5 \mathrm{~A}_{\mathrm{d} 2}^{2}}=-2 \quad \text { VII. } 16
$$

Eqs. VII.15 and VII.16 cannot be solved uniquely for $A_{s} / A_{d 1}, A_{d 2} / A_{d 1}, \delta_{s}^{--\delta} d 1$, and $\delta_{d 2}-\delta_{d 1}$. It should be noted from eq. VII.16 that pure $A_{d 2}$ gives the wrong sign for the left side of the equation. Inspection of eq. VII.16 reveals that if $A_{d 2} \neq 0$ then both $A_{s} \neq 0$ and $A_{d 1} \neq 0$, and that the maximum possible value for $A_{d 2}$ occurs when $A_{d 2}=A_{d 1}$ in which case $A_{s}=1.41 A_{d 1} \cdot A_{d 2}$ can never be the largest amplitude, nor can it be non-zero without requiring both other E1 amplitudes to be non-zero. Thus for the sake of an example we will assume that $\mathrm{A}_{\mathrm{d} 2}=0$.

The polarization measurement at $45^{\circ}$ for neutrons of about $4 \mathrm{MeV}$ energy, the peak of the $\mathrm{C}^{12} \mathrm{giant}$ resonance (21 to $27 \mathrm{MeV}$ photons, 2 to $7 \mathrm{MeV}$ g.s. neutrons) is $\mathrm{P}_{\mathrm{n}}=$ $-0.20 \pm 0.05$, which gives (assuming $A_{d 2}=0$ )

$$
-0.20 \pm 0.05=\frac{1.61 \mathrm{~A}_{\mathrm{S}} \mathrm{A}_{\mathrm{d} 1} \sin \Delta}{3\left(\mathrm{~A}_{\mathrm{s}}^{2}+\mathrm{A}_{\mathrm{d} 1}^{2}\right)+\left(4.24 \mathrm{~A}_{\mathrm{s}} \mathrm{A}_{\mathrm{d} 1} \cos \Delta-1.5 \mathrm{~A}_{\mathrm{d} 1}^{2}\right)(1 / 4)}
$$

VII. 17

where $\Delta=\delta_{\mathrm{dl}}-\delta_{\mathrm{s}}$.

Since $P_{n}\left(90^{\circ}\right) \simeq 0$ the data can be fit with a pure El transition, and the solutions of eqs. VII.16 and VII.17, assuming $A_{d 2}=0$, are

$$
A_{S}=\left(1.79 \begin{array}{r}
+0.41 \\
-0.40
\end{array}\right) A_{d 1}, \Delta=\delta_{d 1}-\delta_{S}=-(129+12)^{\circ}
$$


and

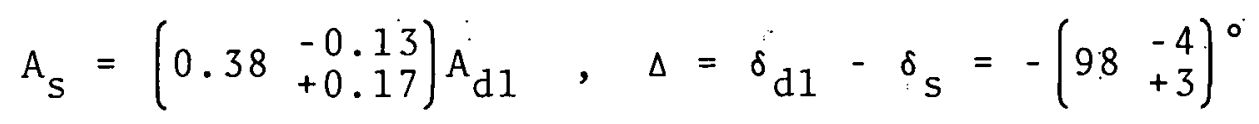

VII. 19

The solutions VII.18 and VII.19 are not the only possible solutions if there are $d$-wave neutrons present with a channel spin of 2. However, all solutions require some s-wave neutrons to be present as otherwise the angular distribution and polarization measurements can not be fit. simultaneously. The conclusion of Verbinski and Courtney (26) that mostly d-wave neutrons are emitted by $\mathrm{C}^{12}\left(\gamma_{0}, \mathrm{n}_{0}\right)$ is incorrect, as a large s-wave can be present(eq. VII.18) and still fit the experiments. The $45^{\circ}$ neutron polarization measurements allow us to conclude that both s-and d-waveneutrons are present.

D. Interpretation of the $\mathrm{Pb}^{208}(\gamma, \mathrm{n})$ Results The $\mathrm{Pb}^{208}\left(\gamma, \mathrm{n}_{\mathrm{O}}\right)$ reaction is a transition from the $0^{+}$ g.s. of $\mathrm{Pb}^{208}$ to the $1 / 2^{-} \mathrm{g} . \mathrm{s}$. of $\mathrm{Pb}^{207}$. The neutrons may have a combination of $s$ - and $d$-waves, as in the case for $\mathrm{O}^{16}\left(\mathrm{r}, \mathrm{n}_{\mathrm{O}}\right)$. For pure $\mathrm{El}$ transitions in $\mathrm{Pb}^{208}$, we have equations for $\frac{d \sigma}{d \Omega}$ and $\overrightarrow{\mathrm{P}}_{\mathrm{n}}$. which are identical to eqs. VII.4 and VII.5. The complete equations for E1, M1 and E2 transitions are also identical to the $0^{16}$ results, and are given in appendix IV.

Mutchler ${ }^{(58)}$ has measured the angular distribution of the photoneutrons from the giant resonance of $\mathrm{Pb}^{208}$ to be

$$
\frac{\mathrm{d} \sigma}{\mathrm{d} \Omega}=\mathrm{a}\left(1.0-0.72 \mathrm{P}_{2}(\cos \theta)\right)
$$


This is for $5.8 \mathrm{MeV}$ neutrons which would arise from a 13.2 $\mathrm{MeV}$ excitation in $\mathrm{Pb}^{208}$ decaying to the g.s. of $\mathrm{Pb}^{207}$. The $45^{\circ}$ polarization of these neutrons is $P_{n}=+0.16 \pm 0.10$. If we use these values in eqs. VII.4 and VII.5 we obtain the two solutions

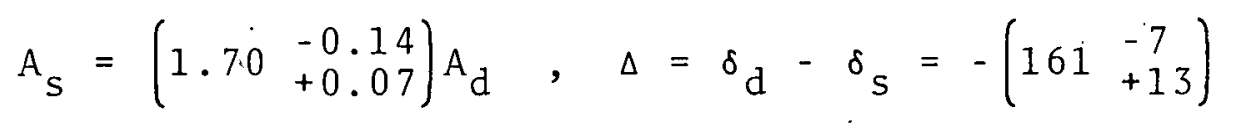

VI I. 21

and

$$
A_{S}=\left(\begin{array}{ll}
0.22 & +0.08 \\
-0.04
\end{array}\right) A_{d}, \Delta=\delta_{d}-\delta_{S}=-\left(144 \begin{array}{l}
-9 \\
+20
\end{array}\right)
$$

VI I . 22

where the errors shown arise only from the uncertainty in the polarization measurement. The $\mathrm{Pb}^{208}(\mathrm{r}, \mathrm{n})$ polarization at $90^{\circ}$ is approximately 0 and together with the angular distribution of Mutchler, which is symmetric about $90^{\circ}$, supports the assumption of a pure E1 excitation. However, the solutions VII.21 and VII.22 are only rough indications of the g.s. neutron $s$ - and d-wave amplitude ratio and relative phases because $\mathrm{Pb}^{207}$ has many low lying excited states and there is probably a good deal of contamination by non-g.s. transition neutrons. 
$-207-$

APPENDIX I

Electronic Circuit Diagrams 

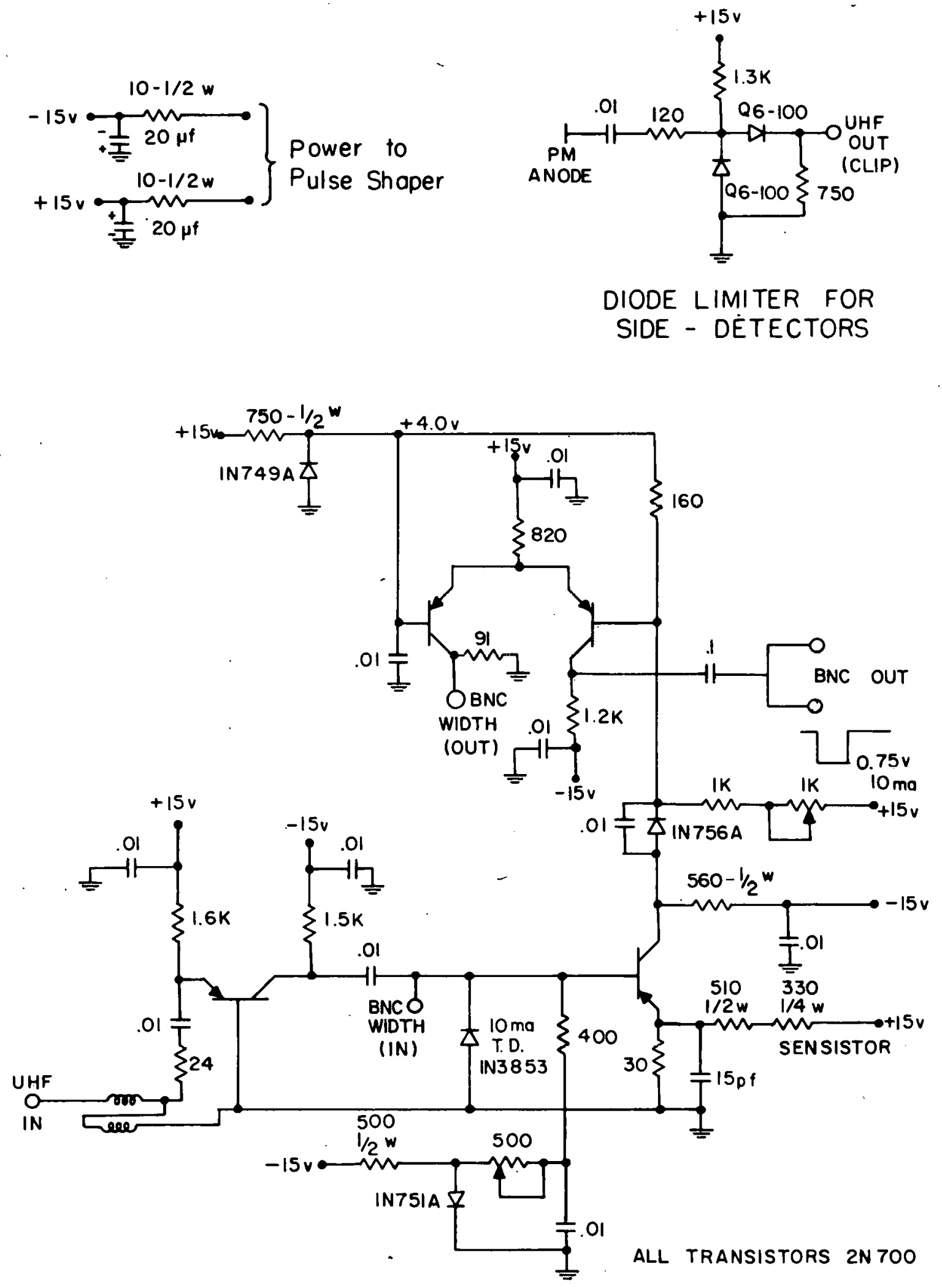

TUNNEL DIODE PULSE SHAPER

(THRESHOLD PULSE SHAPER)

Fig. AI - I 


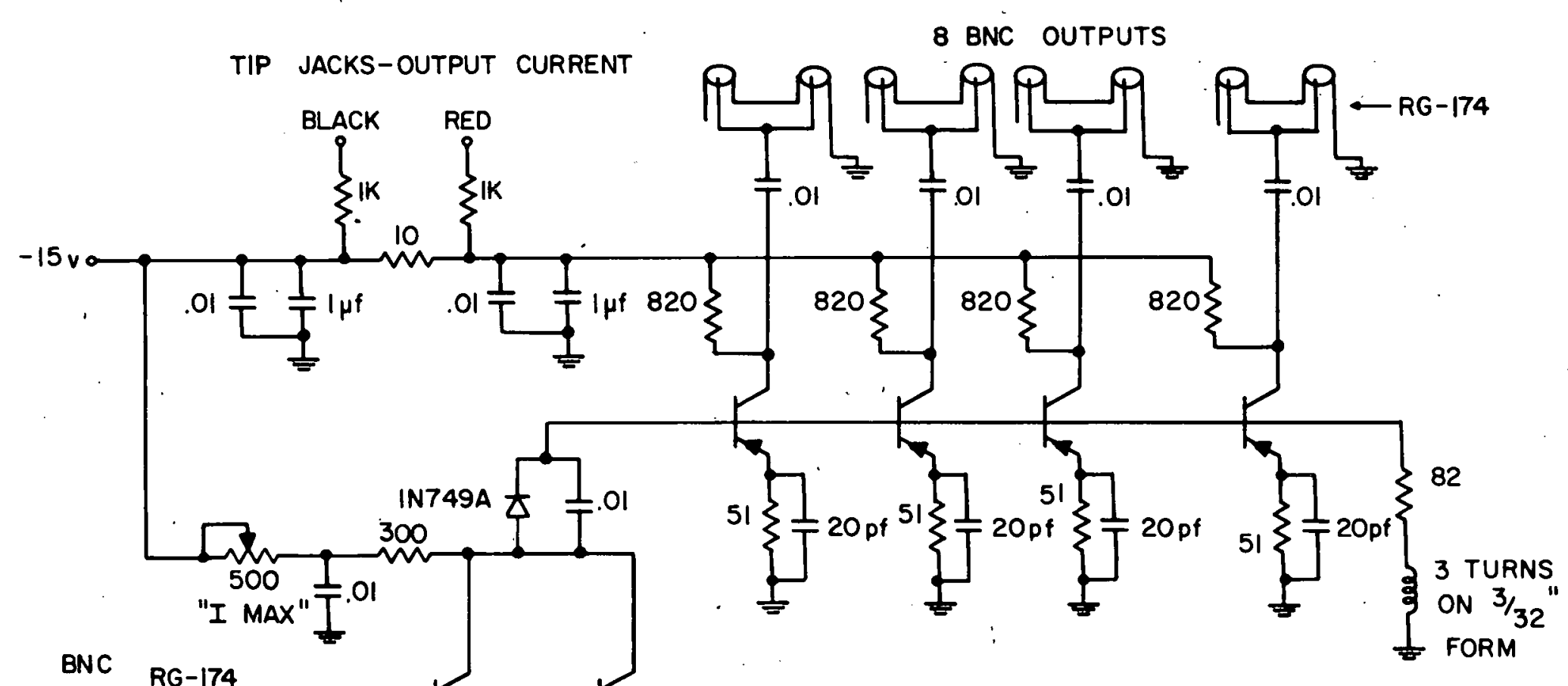

ALL TRANSISTORS 2N700

PULSE FANOUT (BROOKHAVEN TYPE)

Fig. AI - 2 


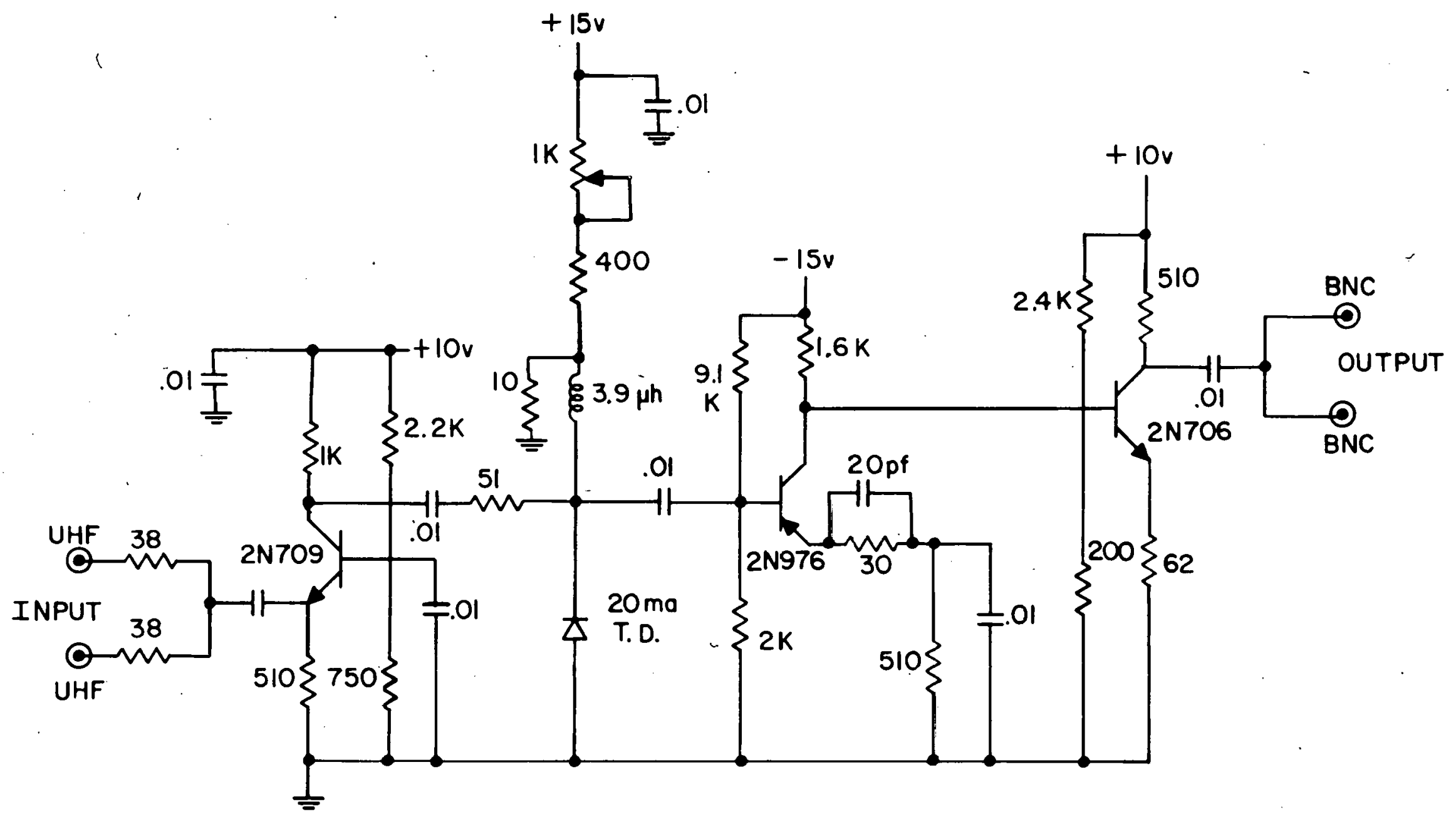

N

TUNNEL DIODE COINCIDENCE

Fig. Ẩ -3 


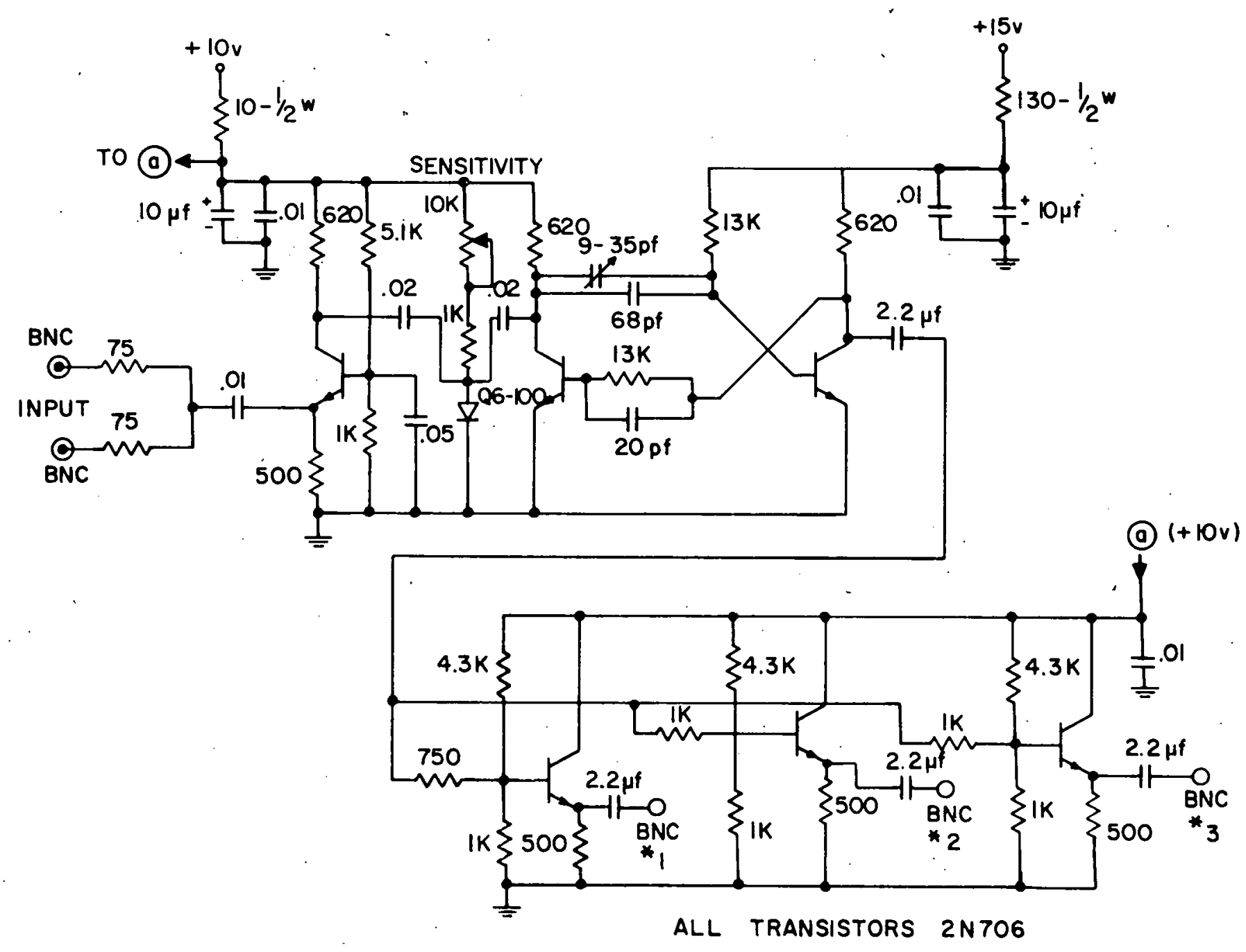

300 NS UNIVIBRATOR

Fig. AI - 4 


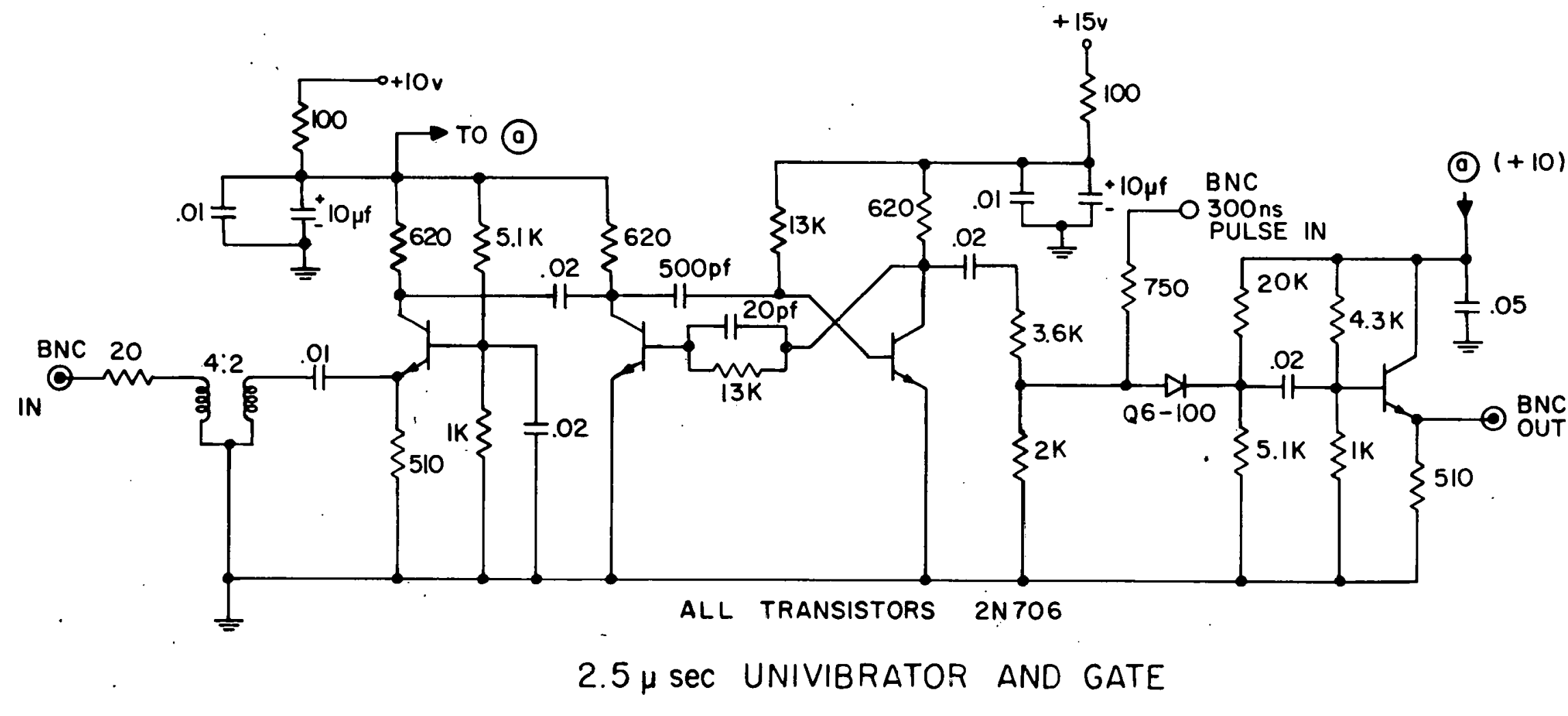

Fig. AI - 5 


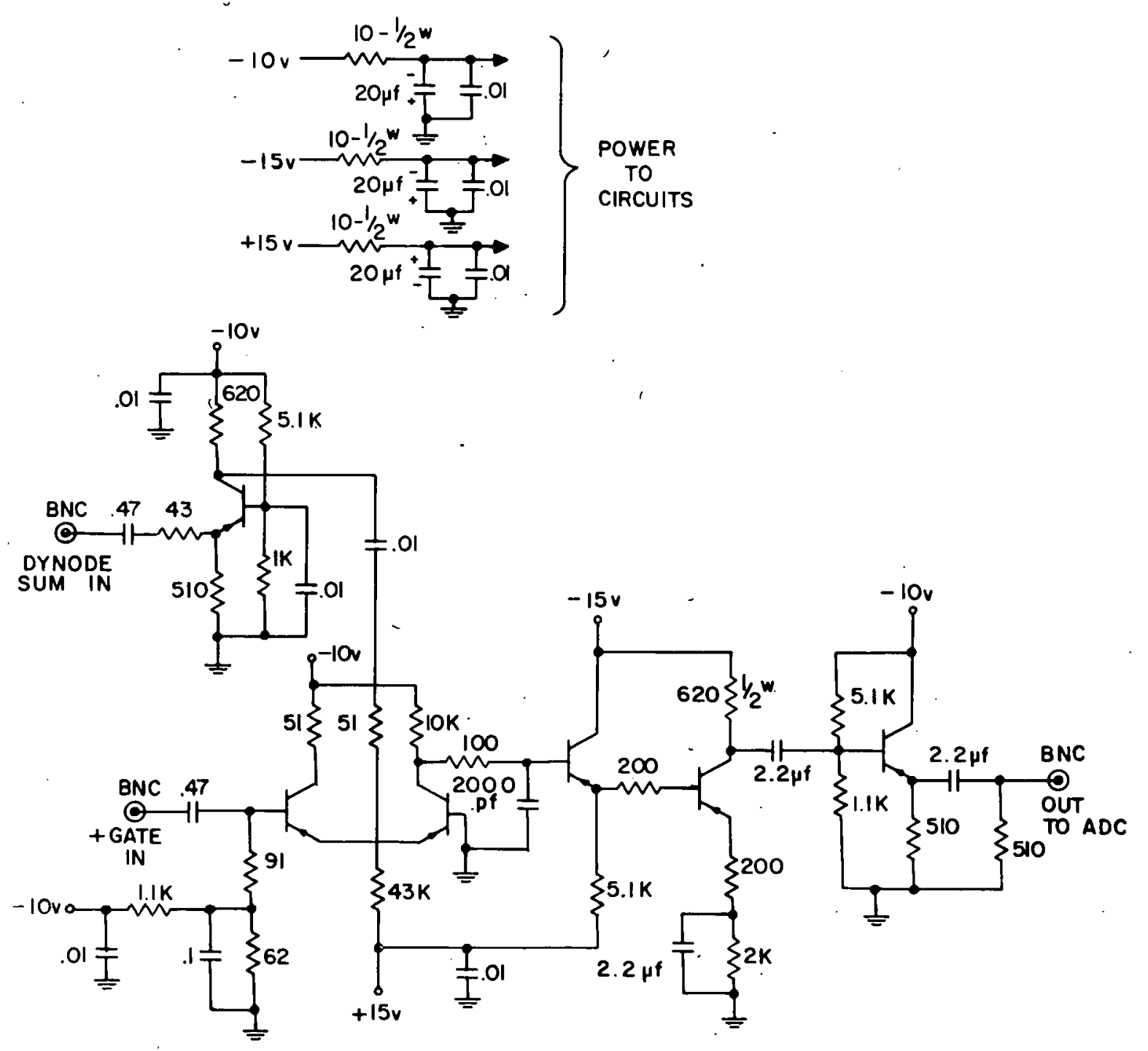

ALL TRANSISTORS 2N976

LINEAR GATE, INTEGRATE AND STRETCH

Fig. AI -6 


\section{APPENDIX I I}

Calculation of $\overline{P_{\mathrm{He}}\left(\theta_{0}, E_{n}\right)}$ and $\left.\overline{S_{\mathrm{He}}\left(\theta_{o}, E_{n}\right.}\right)$.

$\overline{\mathrm{P}_{\mathrm{He}}\left(\theta_{0}, \mathrm{E}_{n}\right)}$ and $\overline{\mathrm{S}_{\mathrm{He}}\left(\theta_{0}, E_{n}\right)}$ are the effective polarization analyzing power and detection efficiency of the polarimeterspectrometer. If a monoenergetic beam of $\mathrm{N}_{\mathrm{n}}$ neutrons $/ \mathrm{cm}^{2}$ of $\mathrm{E}_{\mathrm{n}}$. $\mathrm{MeV}$ and with polarization $\mathrm{P}_{\mathrm{n}}$ were incident on the polarimeter, then a side-detector pair with a central scattering angle $\theta_{0}$ would yield a measured asymmetry of

$$
A_{n}\left(\theta_{0}, E_{n}\right)=P_{n} \overline{P_{H e}\left(\theta_{0}, E_{n}\right)} \quad \text { A.II.1 }
$$

The total number of detected neutrons is given by

$$
\mathrm{N}_{\text {det }}=\mathrm{N}_{\mathrm{n}} \overline{\mathrm{S}_{\mathrm{He}}\left(\theta_{0}, \mathrm{E}_{\mathrm{n}}\right)}
$$

A. I I. 2

The approximately averaged values of $\overline{\mathrm{P}_{\mathrm{He}}\left(\theta_{0}, \mathrm{E}_{\mathrm{n}}\right)}$ and $\overline{\mathrm{S}_{\mathrm{He}}\left(\theta_{0}, \mathrm{E}_{\mathrm{n}}\right)}$ are calculated as follows. Consider the scattering shown in fig. AII-1. Here a neutron with $\vec{k}_{n}$ is scattered in a volume of $\mathrm{H}_{e}, \mathrm{dV}_{\mathrm{He}}(\mathrm{i})$, and is detected in a smail volume of a side-detector, $\mathrm{dV}_{\mathrm{SD}}(j)$. The scattering angle is $\theta_{i j}$ and the distance between the centers of the small volumes is $\mathbf{r}_{i j}$. The number of detected neutrons from this scattering will be $d N_{d e t}=N_{n} \quad \varepsilon \frac{d V_{S D}(j)}{\left(r_{i j}\right)^{2}} n_{H e} d V_{H e}(i) \frac{d \sigma}{d \Omega} H e\left(\theta_{i j}\right)\left[1+P_{n} \hat{n}_{p} \cdot \hat{n}_{s} P_{H e}\left(\theta_{i j}\right)\right]$

A. I I. 3

where

$$
\begin{aligned}
\varepsilon= & \text { the detection efficiency per unit volume of the } \\
& \text { side-detector for neutrons of energy } E_{n}{ }^{\prime} \mathrm{MeV} \text {, where } \\
& E_{n}{ }^{\prime} \text { is the energy of the scattered neutron, } \vec{k}_{n}{ }^{\prime} .
\end{aligned}
$$




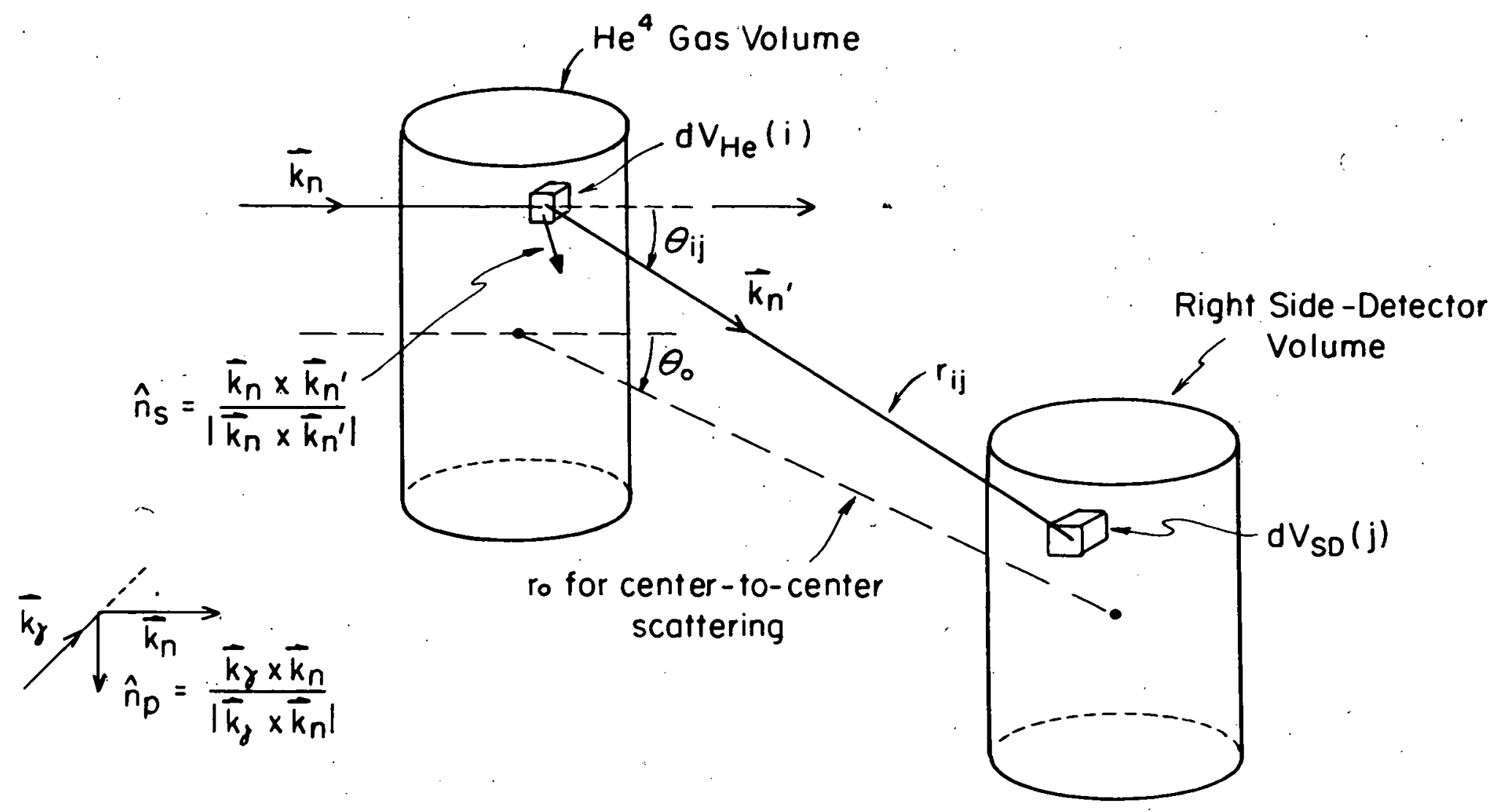

Fig. AII - I 
$\mathrm{n}_{\mathrm{He}}=$ the number of $\mathrm{He}^{4}$ nuclei/ $\mathrm{cm}^{3}$ in the gas chamber. $\frac{d_{\sigma}}{d \Omega} \operatorname{He}\left(\theta_{i j}\right), P_{H e}\left(\theta_{i j}\right)=$ the laboratory differential scattering. cross section and polarizing power for $\mathrm{He}^{4}(\mathrm{n}, \mathrm{n})$ scattering at $1 \mathrm{ab}$ angle $\theta_{i j} \cdot$

$\hat{n}_{p}=\frac{\vec{k}_{\gamma} \times \vec{k}_{n}}{\left|\vec{k}_{\gamma} \times \vec{k}_{n !}\right|}=$ the unit vector along which $P_{n}$ is defined. $\hat{n}_{s}=\frac{\vec{k}_{n} \times \vec{k}_{n}^{\prime}}{\left|\vec{k}_{n} \times \vec{k}_{n}^{\prime}\right|}=$ the unit vector perpendicular to the $\mathrm{He}^{4}(n, n)$ scattering, and in which direction $\mathrm{P}_{\mathrm{He}}\left(\theta_{i j}\right)$ is defined.

If we choose the right side-detector, i.e. the one at central scattering angle $+\theta_{0}$, then $\hat{n}_{p} \cdot \hat{n}_{s} \simeq+1$ and by summing over all volume elements $d V_{H e}(i)$ and $d_{S D}(j)$ we obtain

$$
\mathrm{N}_{\text {det }_{\mathrm{R}}}=\frac{\mathrm{N}_{\mathrm{n}}}{2} \overline{\mathrm{S}_{\mathrm{He}}\left(\theta_{0}, \mathrm{E}_{\mathrm{n}}\right)}\left[1+\mathrm{P}_{\mathrm{n}} \overline{\mathrm{P}_{\mathrm{He}}\left(\theta_{0}, \mathrm{E}_{\mathrm{n}}\right)}\right]
$$

\section{A. I I. 4}

where

$$
\begin{aligned}
& \left.\overline{S_{H e}\left(\theta_{0}, E_{n}\right)}=2 \sum_{i j}^{\prime} \varepsilon \frac{d V_{S D}(j)}{\left(r_{i j}\right)^{2}} n_{H e} d V_{H e}(i) \frac{d \sigma}{d \Omega} H e \theta_{i j}\right)_{\frown} \\
& \text { A.II. } 5 \\
& \overline{P_{H e}\left(\theta_{0}, E_{n}\right)}=\frac{2 \sum_{i j}^{\prime} \varepsilon \frac{d V_{S D}(j)}{\left(r_{i j}\right)^{2}} n_{H e} d V_{H e}(i) \frac{d \sigma}{d \Omega} \operatorname{He}\left(\theta_{i j}\right) \hat{n}_{p} \cdot \hat{n}_{S} P_{H e}\left(\theta_{i j}\right)}{\overline{S_{H e}\left(\theta_{o}, E_{n}\right)}}
\end{aligned}
$$

The prime in $\sum_{i j}^{\prime}$ means that the $\mathrm{He}^{4}(n, n)$ scattering in $\mathrm{dV}_{\mathrm{He}}(i)$ and the neutron detection in $\mathrm{dV}_{\mathrm{S} D}(j)$ must obey the timing conditions set by the coincidence circuits.

The scattering towards the left side-detector $\left(-\theta_{0}\right)$ is illustrated in fig. AII-2 and has $\hat{n}_{p} \cdot \hat{n}_{s}=-1$. Because of the symmetry in the location of the right and left side-detectors the number 


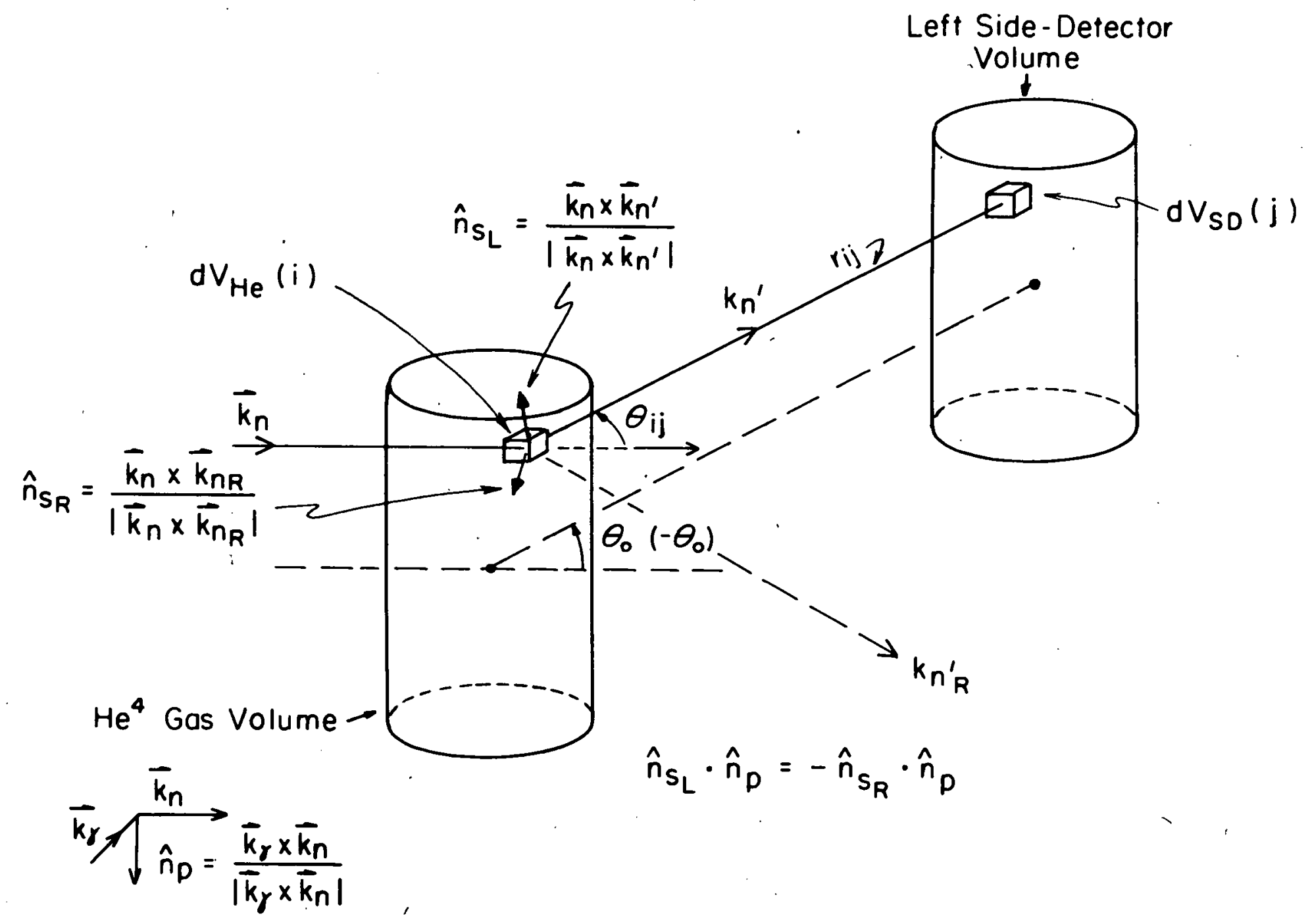

Fig. AII - 2 
detected by the left side-detector is

$$
N_{\text {detL }}=\frac{N_{n}}{2}-\overline{S_{H e}\left(\theta_{0}, E_{n}\right)}\left[1-P_{n} \overline{P_{H e}\left(\theta_{0}, E_{n}\right)}\right]
$$

By combining eqs. A.II.4 and A.II.6 we obtain, using eqs. A.II.1 and A.II.2,

$$
\begin{aligned}
A_{n}\left(\theta_{0}, E_{n}\right) & =\frac{N_{\operatorname{detR}}-N_{\operatorname{det} L}}{N_{\operatorname{det} R}+N_{\operatorname{det} L}} \\
& =P_{n} \overline{P_{H e}\left(\theta_{0}, E_{n}\right)}
\end{aligned}
$$

and

$$
\begin{aligned}
N_{\text {det }} & =N_{\text {detR }}+N_{\text {det } L} \\
& =N_{n} \overline{S_{H e}\left(\theta_{0}, E_{\text {on }}\right)}
\end{aligned}
$$

A. I I. 8

with eq. A.II.5 giving the values for $\overline{\mathrm{P}_{\mathrm{He}}\left(\theta_{0}, E_{n}\right)}$ and $\overline{\mathrm{S}_{\mathrm{He}}\left(\theta_{0}, E_{n}\right)}$.

The sums in eq. A.II.5 were calculated using the phase shifts of Hoop and Barschall to find the values of $\frac{d}{d} \sigma_{\Omega} H e \cdot\left(\theta_{i j}\right)$ and $\mathrm{P}_{\mathrm{He}}\left(\theta_{i j}\right)$. The detection efficiencies, $\varepsilon$, were taken to be proportional to the calculations of Verbinski for a 5 " diameter and 5" long cylinder of NE 212 plastic scintillator. Fig. AII-3 shows a plot of $\varepsilon_{\text {tot }}=\varepsilon \times V_{\text {tot }} v s$. the energy of the neutron incident on the side-detector for a detection threshold of 0.75 MeV. $V_{\text {tot }}$ is the total detection volume of the side-detectors and Eot is the total neutron detection efficiency of the sidedetector.

The results of the calculations using eqs. A.II.5 are shown in figs.AII-4. Each sub-figure lists the values of $\theta_{0}$, $r_{0}$ (the center-to-center distance of the gas-counter and side-detector), the neutron detection threshold of the side-detectors, and the 
coincidence circuit timing. All calculations were made assuming that 700 psia of helium were in the gas chamber. 


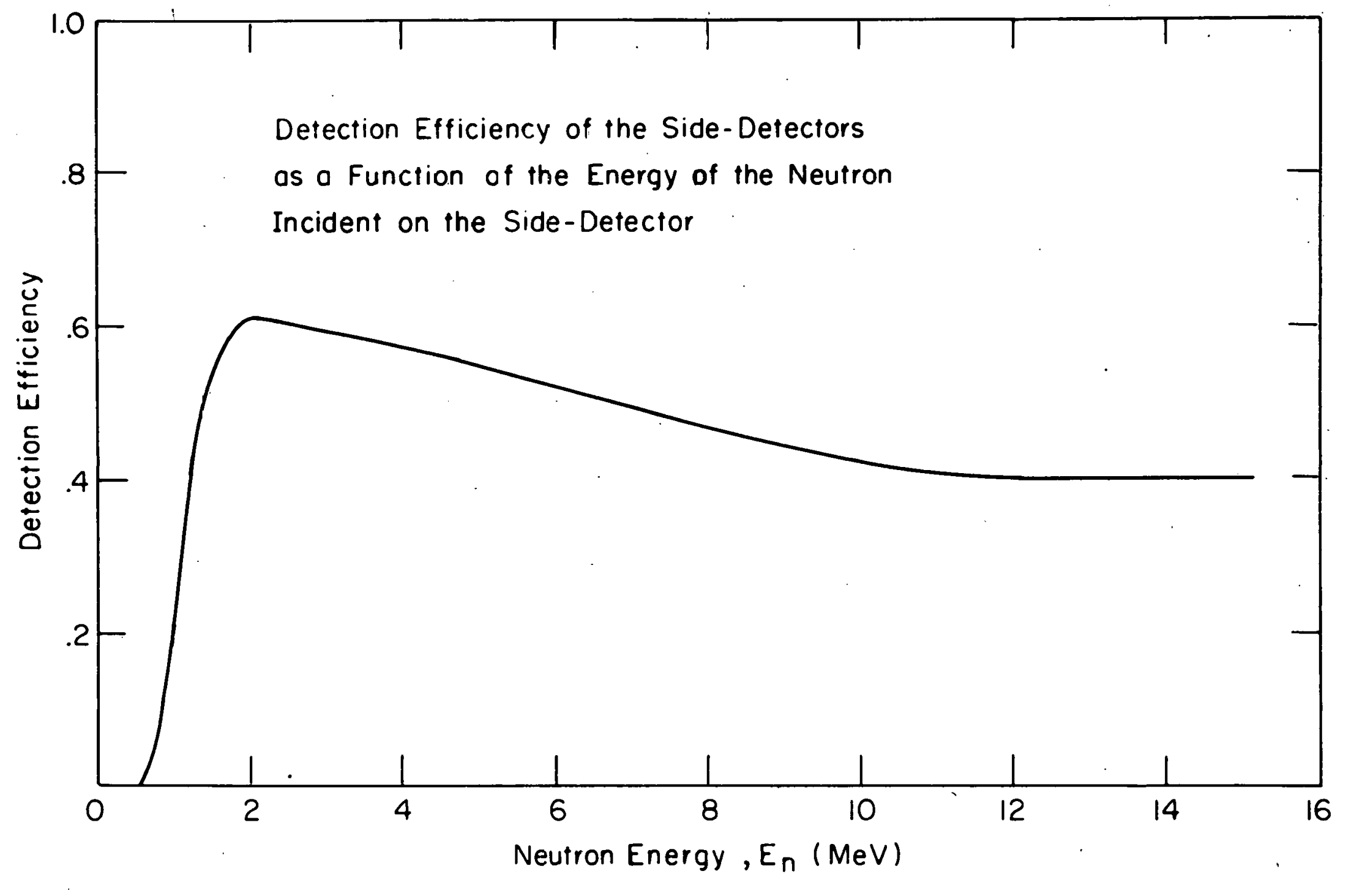



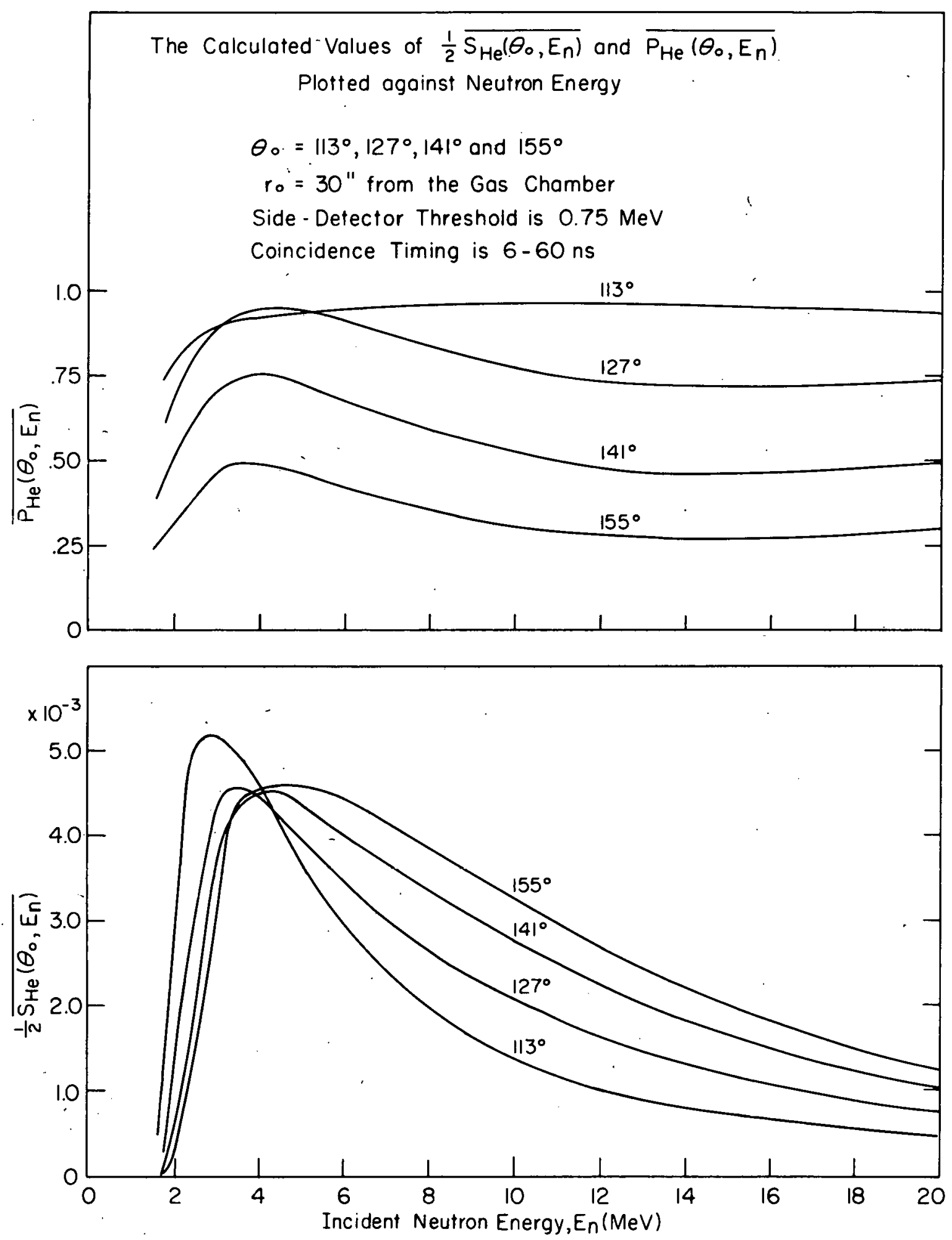


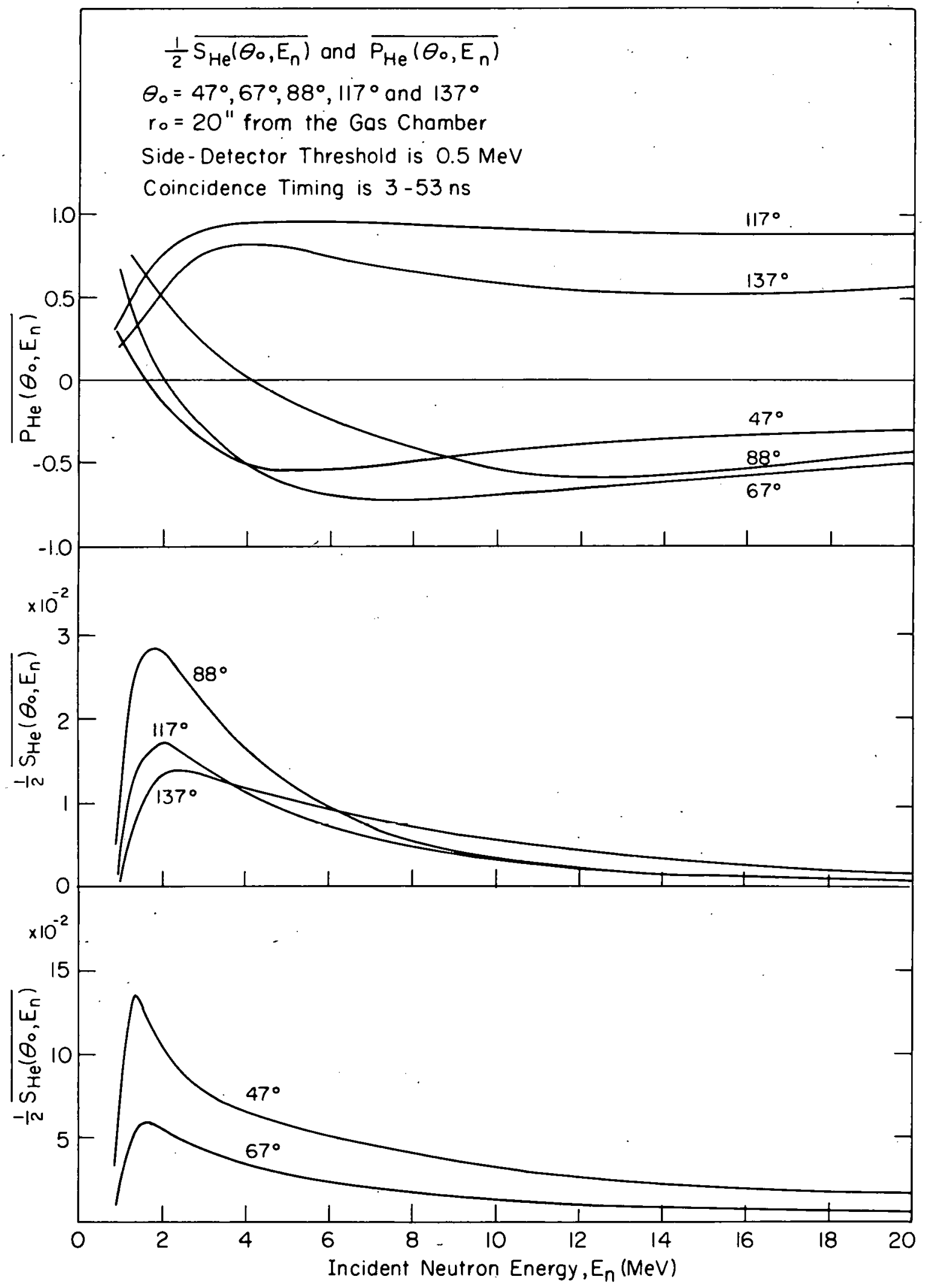




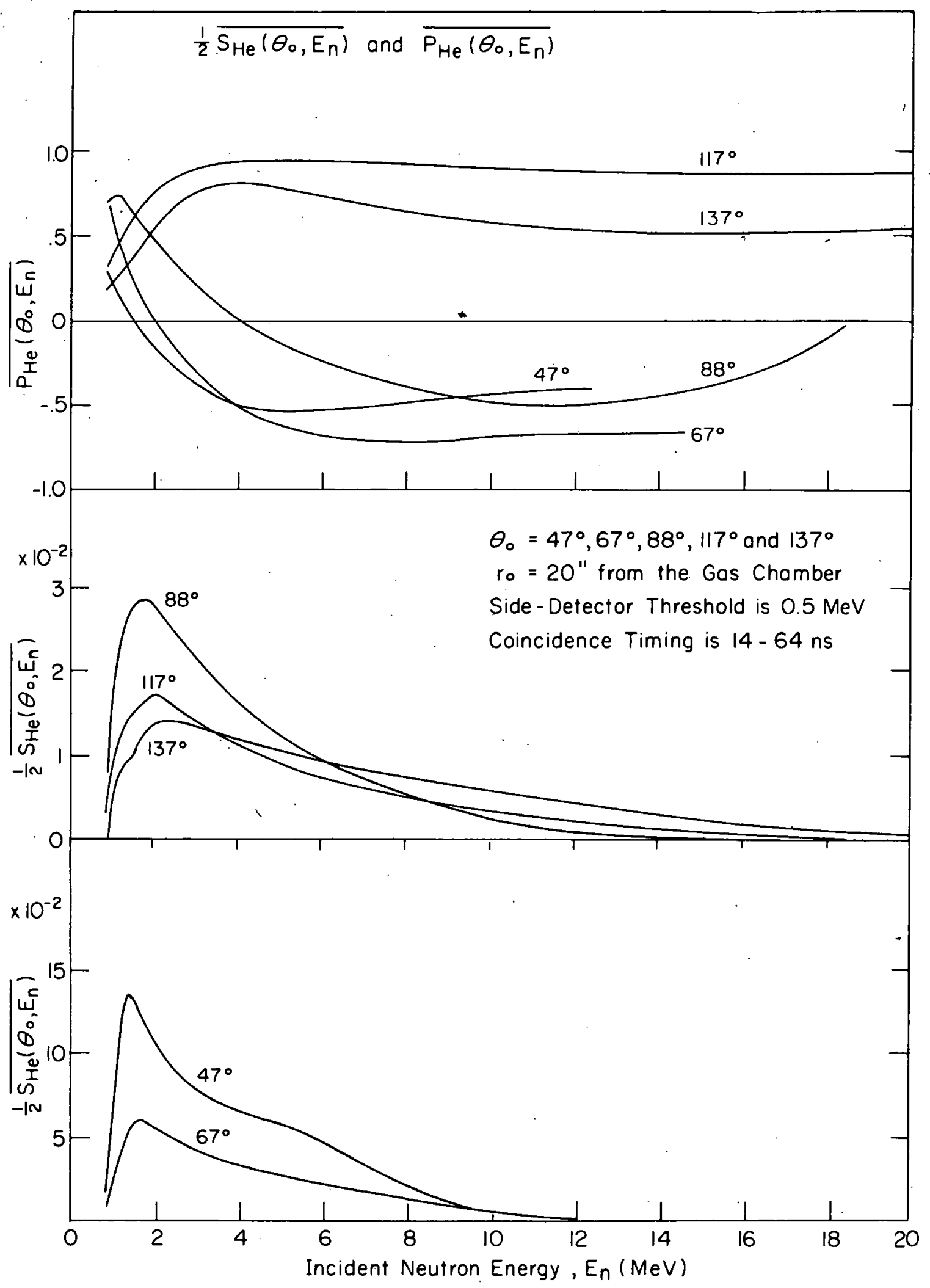




\section{APPENDIX III}

Equations for $\Delta \mathrm{P}_{0^{16}}$ and $\Delta \mathrm{N}_{0^{16}}$

From chapter IV we have eqs. IV.34 and IV.35

$$
k_{c}=\frac{\bar{N}_{c} I_{o x} m_{o x}}{\bar{N}_{o x} I_{c} m_{c}}
$$

and

$$
\begin{aligned}
& \mathrm{P}_{016}=\frac{\overline{\mathrm{P}}_{\mathrm{OX}}-\mathrm{k}_{\mathrm{c}} \overline{\mathrm{P}}_{\mathrm{C}}}{1-\mathrm{k}_{\mathrm{c}}} \\
& \mathrm{N}_{0^{16}}=\overline{\mathrm{N}}_{\mathrm{OX}}\left(1-\mathrm{k}_{\mathrm{c}}\right)
\end{aligned}
$$

Also

$$
\begin{aligned}
& \Delta \mathrm{P}_{0}{ }^{16}=\left(\left(\Delta \mathrm{P}_{0^{16}} \sigma_{s t}\right)^{2}+\left(\Delta \mathrm{P}_{0^{16} \sigma_{\text {sys }}}\right)^{2}\right)^{1 / 2} . \\
& \Delta N_{0} 16=\left(\left(\Delta N_{0} 16_{s t}\right)^{2}+\left(\Delta N_{0} 16_{s y s}\right)^{2}\right)^{1 / 2}
\end{aligned}
$$

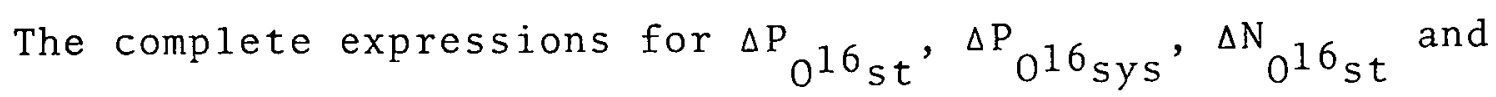
$\Delta{ }_{0} 16_{\text {sys }}$ in terms of quantities defined in chapter IV are

$$
\begin{aligned}
\Delta \mathrm{P}_{016_{s t}=} & \frac{1}{1-\mathrm{k}_{\mathrm{c}}}\left(\left(\Delta \overline{\mathrm{P}}_{\mathrm{ox}}\right)^{2}+\left(\mathrm{k}_{\mathrm{c}}\left(\Delta \overline{\mathrm{P}}_{\mathrm{c}_{\mathrm{st}}}\right)\right)^{2}\right. \\
& \left.+\left(\frac{\overline{\mathrm{P}}_{\mathrm{ox}}-\overline{\mathrm{P}}_{\mathrm{c}}}{1-\mathrm{k}_{\mathrm{c}}}\right)^{2}\left(\Delta \mathrm{k}_{\mathrm{c}_{\mathrm{st}}}\right)^{2}\right)^{1 / 2} \\
\Delta \mathrm{P}_{0} 16_{\text {sys }}= & \frac{1}{1-\mathrm{k}_{\mathrm{c}}}\left(\left(\Delta \overline{\mathrm{P}}_{\mathrm{ox}}\right)_{\text {sys }}^{2}+\left(\mathrm{k}_{\mathrm{c}}\left(\Delta \overline{\mathrm{P}}_{\mathrm{c}_{\text {sys }}}\right)\right)^{2}\right.
\end{aligned}
$$


$-225-$

$$
\left.+\left(\frac{\bar{P}_{o x}-\bar{P}_{c}}{1-k_{c}}\right)^{2}\left(\Delta k_{c_{s y s}}\right)^{2}\right)^{1 / 2}
$$

$$
\begin{aligned}
& \left.\Delta \mathrm{N}_{0} 16_{\mathrm{st}} \doteq\left(\left(1-\mathrm{k}_{\mathrm{c}}\right)^{2}\left(\Delta \overline{\mathrm{N}}_{\mathrm{ox}}\right)_{\mathrm{st}}\right)^{2}+\left(\overline{\mathrm{N}}_{\mathrm{ox}}\right)^{2}\left(\Delta \mathrm{k}_{\mathrm{c}_{\mathrm{st}}}\right)^{2}\right)^{1 / 2} \\
& \Delta \mathrm{N}_{0^{16}} \sigma_{\text {sys }}=\left(\left(1-k_{c}\right)^{2}\left(\Delta \bar{N}_{o x}{ }_{\text {sys }}\right)^{2}+\left(\bar{N}_{o x}\right)^{2}\left(\Delta k_{c_{\text {sys }}}\right)^{2}\right)^{1 / 2}
\end{aligned}
$$

where

$$
\begin{aligned}
& \Delta k_{c_{s t}}=k_{c}\left(\left(\frac{\Delta \bar{N}_{C_{S t}}}{\bar{N}_{c}}\right)^{2}+\left(\frac{\Delta \bar{N}_{O x_{S t}}}{\bar{N}_{O x}}\right)^{2}+\left(\frac{\Delta I}{I_{C}}\right)^{2}\right. \\
& \left.+\left(\frac{\Delta I_{o x}}{I_{o x}}\right)^{2}\right)^{1 / 2}
\end{aligned}
$$

and

$$
k_{c_{\text {sys }}}=k_{c}\left(\left(\frac{\Delta \bar{N}_{c_{\text {sys }}}}{\bar{N}_{c}}\right)^{2}+\left(\frac{\Delta \bar{N}_{\text {ox }}}{\bar{N}_{\text {ox }}}\right)_{\text {sys }}\right)^{2 / 2}
$$




\section{APPENDIX IV}

Formulas for the Angular Dependence of the $(r, n)$

Cross Section and Neutron Polarization,

Including E1, M1 and E2 Transitions

Here are presented the results of eqs. VII.1 and VII.2 for $\frac{d \sigma}{d \Omega}$ and $\frac{d \vec{P}}{d \Omega}$. The actual neutron polarization defined in this thesis is.

$$
\overrightarrow{\mathrm{P}}_{\mathrm{n}}=\frac{1}{j_{1}}\left[\frac{\left(\frac{\mathrm{d} \overrightarrow{\mathrm{p}}}{\mathrm{d} \Omega}\right)}{\left(\frac{\mathrm{d \sigma}}{\mathrm{d} \Omega}\right)}\right]
$$

AIV. 1

where this is eq. VII.3, and $j_{1}=1 / 2$ for neutrons. We assume only E1, M1 and E2 t'ransitions, and include all interference terms.

For $0^{16}\left(r, n_{0}\right) 0^{15}$ transitions the final nuclear state is $1 / 2^{-}$and from eq. VII.l we obtain(the notation is made more obvious by setting $\left(\ell s \alpha^{\prime}\left|R_{\Pi}^{J}\right| p g \alpha\right)=A_{(\ell)}(s,(p g))$, where $(\ell)=s, p, d$, etc. for $\ell=0,1,2$, ctc., and $(\mathrm{pg})=E 1$, M1, or E2. $J^{I l}$ is completely determined by $(\mathrm{pg})$ since the initial nuclear spin-parity is $0^{+}$)

$$
\begin{aligned}
\frac{d \sigma}{d \Omega}= & \frac{\pi^{2}}{8}\left(\left[3 \left(\left|A_{p}(0, M 1)\right|^{2}+\left|A_{p}(1, M 1)\right|^{2}+\left|A_{s}(1, E 1)\right|^{2}\right.\right.\right. \\
& \left.\left.+\left|A_{d}(1, E 1)\right|^{2}\right)+5\left(\left|A_{p}^{*}(1, E 2)\right|^{2}+\left|A_{f}(1, E 2)\right|^{2}\right)\right](1) \\
& +2\left[-3.67 \operatorname{Re}\left(A_{S}^{*}(1, E 1) A_{p}(1, M 1)\right)+4.74 \operatorname{Re}\left(A_{S}^{*}(1, E 1)\right.\right. \\
& \left.\times A_{p}(1, E 2)\right)-2.60 \operatorname{Re}\left(A_{d}^{*}(1, E 1) A_{p}(1, M 1)\right)-0.67 \\
& \left.\times \operatorname{Re}\left(A_{d}^{*}(1, E 1) A_{p}(1, E 2)\right)+4.93 \operatorname{Re}\left(A_{d}^{*}(1, E 1) A_{f}(1, E 2)\right)\right] \\
& \times(\cos \theta)+\left[-3\left|A_{p}(0, M 1)\right|^{2}-1.5\left|A_{d}(1, E 1)\right|^{2}\right.
\end{aligned}
$$




$$
\begin{aligned}
& +1.5\left|A_{p}(1, M 1)\right|^{2}+2.5\left|A_{p}(1, E 2)\right|^{2}+2.86\left|A_{f}(1, E 2)\right|^{2} \\
& +2\left[2.12 \operatorname{Re}\left(A_{s}^{*}(1, E 1) A_{d}(1, E 1)\right)-5.81 \operatorname{Re}\left(A_{p}^{*}(1, M 1)\right.\right. \\
& \left.\times A_{p}(1, E 2)\right)-4.74 \operatorname{Re}\left(A_{p}^{*}(1, M 1) A_{f}(1, E 2)\right)-0.87 \\
& \left.\left.\times \operatorname{Re}\left(A_{p}^{*}(1, E 2) A_{f}(1, E 2)\right)\right]\right]\left(\frac{3 \cos 2 \theta+1}{4}\right)+2[3.87 \\
& \times \operatorname{Re}\left(A_{s}^{*}(1, E 1) A_{f}(1, E 2)\right)+4.02 \operatorname{Re}\left(A_{d}^{*}(1, E 1) A_{p}(1, E 2)\right) \\
& \left.-2.19 \operatorname{Re}\left(A_{d}^{*}(1, E 1) A_{f}(1, E 2)\right)\right]\left(\frac{5 \cos 3 \theta+3 \cos \theta}{2}\right) \\
& +\left[14 \operatorname{Re}\left(A_{p}^{*}(1, E 2) A_{f}(1, E 2)\right)-2.86\left|A_{f}(1, E 2)\right|^{2}\right] \\
& \left.\times\left(\frac{35 \cos 4 \theta+20 \cos 2 \theta+9}{64}\right)\right\}
\end{aligned}
$$

Eq. VII.2 yields

$$
\begin{aligned}
\frac{d \vec{P}}{d \Omega}= & \frac{\vec{k}_{\gamma} \times \vec{k}_{n}}{\left|\vec{k}_{\gamma} \times \vec{k}_{n}\right|} t^{2}(0.22)\left(2 \left[0.87 \operatorname{Re}\left(i A_{p}^{*}(0, M 1) A_{s}(1, E 1)\right)\right.\right. \\
& +0.61 \operatorname{Re}\left(i A_{p}^{*}(0, M 1) A_{d}(1, E 1)\right)-0.61 \operatorname{Re}\left(i A_{s}^{*}(1, E 1)\right. \\
& \left.\times A_{p}(1, M 1)\right)+0.79 \operatorname{Re}\left(i A_{s}^{*}(1, E 1) A_{p}(1, E 2)\right)+0.87 \\
& \times \operatorname{Re}\left(i A_{d}^{*}(1, E 1) A_{p}(1, M 1)\right)-0.45 \operatorname{Re}\left(i A_{d}^{*}(1, E 1) A_{p}(1, E 2)\right) \\
& \left.-0.82 \operatorname{Re}\left(i A_{d}^{*}(1, E 1) A_{f}(1, E 2)\right)\right](-\sqrt{374} s i n \theta) \\
& +2\left[0.47 \operatorname{Re}\left(i A_{p}^{*}(0, M 1) A_{p}(1, M 1)\right)+0.61 \operatorname{Re}\left(i A_{p}^{*}(0, M 1)\right.\right. \\
& \left.\times A_{p}(1, E 2)\right)+0.5 \operatorname{Re}\left(i A_{p}^{*}(0, M 1) A_{f}(1, E 2)\right)-0.47 \\
& \times \operatorname{Re}\left(i A_{s}^{*}(1, E 1) A_{d}(1, E 1)\right)+0.87 \operatorname{Re}\left(i A_{p}^{*}(1, M 1) A_{p}(1, E 2)\right) \\
& -1.06 \operatorname{Re}\left(i A_{p}^{*}(1, M 1) A_{f}(1, E 2)\right)+0.33 \operatorname{Re}\left(i A_{p}^{*}(1, E 2)\right. \\
& \left.\left.\times A_{f}(1, E 2)\right)\right](-\sqrt{15716} \mathrm{sin} 2 \theta)+2\left[-0.69 \operatorname{Re}\left(i A_{s}^{*}(1, E 1)\right.\right.
\end{aligned}
$$




$$
\begin{aligned}
& \left.\times A_{f}(1, E 2)\right)+0.72 \operatorname{Re}\left(i A_{d}^{*}(1, E 1) A_{p}(1, E 2)\right)+0.10 \\
& \left.\times \operatorname{Re}\left(i A_{d}^{*}(1, E 1) A_{f}(1, E 2)\right)\right](-\sqrt{21 / 512}(5 \sin 3 \theta+\sin \theta)) \\
& +2\left[-1.06 \operatorname{Re}\left(i A_{p}^{*}(1, E 2) A_{f}(1, E 2)\right)\right](-\sqrt{45 / 2048} \\
& \times(7 \sin 4 \theta+2 \sin 2 \theta))\}
\end{aligned}
$$

From eqs. AIV.1, AIV.2 and AIV.3 we may obtain the neutron polarization explicitly. These results also apply to $\mathrm{Pb}^{208}\left(\gamma_{,} \mathrm{n}_{\mathrm{o}}\right)$, since the same initial and final nuclear spinparity states are involved.

For $C^{12}\left(\gamma, n_{0}\right) C^{11}$ transitions the final state of the nucleus is $3 / 2^{-}$, and from eq. VII.1 we obtain

$$
\begin{aligned}
& \frac{\mathrm{d} \sigma}{\mathrm{d} \Omega}=\frac{\pi^{2}}{8}\left\{3 \left[\left|\mathrm{~A}_{\mathrm{s}}(1, \mathrm{E} 1)\right|^{2}+\left|\mathrm{A}_{\mathrm{d}}(1, \mathrm{E} 1)\right|^{2}+\left|\mathrm{A}_{\mathrm{p}}(1, M 1)\right|^{2}\right.\right. \\
& \left.+\left|A_{d}(2, E 1)\right|^{2}+\left|A_{p}(2, M 1)\right|^{2}+\left|A_{f}(2, M 1)\right|^{2}\right]+5 \\
& \times\left[\left|A_{p}(1, E 2)\right|^{2}+\left|A_{f}(1, E 2)\right|^{2}+\left|A_{p}(2, E 2)\right|^{2}+\left|A_{f}(2, E 2)\right|^{2}\right] \\
& +2\left[-3.67 \operatorname{Re}\left(A_{S}^{*}(1, E 1) A_{p}(1, M 1)\right)+4.74 \operatorname{Re}\left(A_{S}^{*}(1, E 1)\right.\right. \\
& \left.\times A_{p}(1, E 2)\right)-2.60 \operatorname{Re}\left(A_{d}^{*}(1, E 1) A_{p}(1, M 1)\right)-0.67 \\
& \times \operatorname{Re}\left(A_{d}^{*}(1, E 1) A_{p}(1, E 2)\right)+4.93 \operatorname{Re}\left(A_{d}^{*}(1, E 1) A_{f}(1, E 2)\right) \\
& -3.49 \operatorname{Re}\left(A_{d}^{*}(2, E 1) A_{p}(2, M 1)\right)-2.85 \operatorname{Re}\left(A_{d}^{*}(2, E 1) A_{f}(2, M 1)\right) \\
& -2.01 \operatorname{Re}\left(A_{d}^{*}(2, E 1) A_{p}(2, E 2)\right)+4.02 \operatorname{Re}\left(A_{d}^{*}(2, E 1)\right. \\
& \left.\left.\times A_{f}(2, E 2)\right)\right] \cos \theta+\left[-1.5\left|A_{d}(1, E 1)\right|^{2}+1.5\left|A_{p}(1, M 1)\right|^{2}\right. \\
& +2.5\left|\mathrm{~A}_{\mathrm{p}}(1, \mathrm{E} 2)\right|^{2}+2.86\left|\mathrm{~A}_{\mathrm{f}}(1, \mathrm{E} 2)\right|^{2}+1.5\left|\mathrm{~A}_{\mathrm{d}}(2, \mathrm{E} 1)\right|^{2} \\
& -0.3\left|A_{p}(2, M 1)\right|^{2}-1.2\left|A_{f}(2, M 1)\right|^{2}-2.5\left|A_{p}(2, E 2)\right|^{2}
\end{aligned}
$$




$$
\begin{aligned}
& +0.71\left|A_{f}(2, E 2)\right|^{2}+2\left[2.12 \operatorname{Re}\left(A_{S}^{*}(1, E 1) A_{d}(1, E 1)\right)\right. \\
& -5.81 \operatorname{Re}\left(A_{p}^{*}(1, M 1) A_{p}(1, E 2)\right)-4.74 \operatorname{Re}\left(A_{p}^{*}(1, M 1) A_{f}(1, E 2)\right) \\
& -0.87 \operatorname{Re}\left(A_{p}^{*}(1, E 2) A_{f}(1, E 2)\right)+2.20 \operatorname{Re}\left(A_{p}^{*}(2, M 1) A_{f}(2, M 1)\right) \\
& +2.60 \operatorname{Re}\left(A_{p}^{*}(2, M 1) A_{p}(2, E 2)\right)-5.20 \operatorname{Re}\left(A_{f}^{*}(2, M 1) A_{f}(2, E 2)\right) \\
& +2.12 \operatorname{Re}\left(A_{f}^{*}(2, M 1) A_{p}(2, E 2)\right)-4.24 \operatorname{Re}\left(A_{f}^{*}(2, M 1) A_{f}(2, E 2)\right) \\
& \left.\left.-2.14 \operatorname{Re}\left(A_{p}^{*}(2, E 2) A_{f}(2, E 2)\right)\right]\right]\left(\frac{3 \cos 2 \theta+1}{4}\right)+2[3.87 \\
& \times \operatorname{Re}\left(A_{S}^{*}(1, E 1) A_{f}(L, E 2)\right)+4.02 \operatorname{Re}\left(A_{d}^{*}(1, E 1) A_{p}(1, E 2)\right) \\
& -2.19 \operatorname{Re}\left(A_{d}^{*}(1, E 1) A_{f}(1, E 2)\right)-1.34 \operatorname{Re}\left(A_{d}^{*}(2, E 1) A_{p}(2, E 2)\right) \\
& \left.+2.68 \operatorname{Re}\left(A_{d}^{*}(2, E 1) A_{f}(2, E 2)\right)\right]\left(\frac{5 \cos 3 \theta+3 \cos \theta}{2}\right)-[2.86 \\
& \times\left|A_{f}(1, E 2)\right|^{2}+4.29\left|A_{f}(2, E 2)\right|^{2}+14 \operatorname{Re}\left(A_{p}^{*}(1, E 2) A_{f}(1, E 2)\right) \\
& \left.\left.-5.71 \operatorname{Re}\left(A_{p}^{*}(2, E 2) A_{f}(2, E 2)\right)\right]\left(\frac{35 \cos 4 \theta+20 \cos 2 \theta+9}{64}\right)\right\}
\end{aligned}
$$

AIV. 4

Eq. VII. 2 yields

$$
\begin{aligned}
\frac{d \vec{P}}{d \Omega}= & \frac{\vec{k}_{\gamma} \times \vec{k}_{n}}{\left|\vec{k}_{\gamma} \times \vec{k}_{n}\right|} \pi^{2}(0.22)\left\{2 \left[0.31 \operatorname{Re}\left(i A_{s}^{*}(1, E 1) A_{p}(1, M 1)\right)-0.40\right.\right. \\
& \times \operatorname{Re}\left(i A_{S}^{*}(1, E 1) A_{p}(1, E 2)\right)-0.68 \operatorname{Re}\left(i A_{s}^{*}(1, E 1) A_{p}(2, M 1)\right) \\
& -1.19 \operatorname{Re}\left(i A_{s}^{*}(1, E 1) A_{p}(2, E 2)\right)-0.43 \operatorname{Re}\left(i A_{d}^{*}(1, E 1) A_{p}(1, M 1)\right) \\
& +0.22 \operatorname{Re}\left(i A_{d}^{*}(1, E 1) A_{p}(1, E 2)\right)+0.41 \operatorname{Re}\left(i A_{d}^{*}(1, E 1) A_{f}(1, E 2)\right) \\
& +0.39 \operatorname{Re}\left(i A_{d}^{*}(1, E 1) A_{p}(2, M 1)\right)+0.71 \operatorname{Re}\left(i A_{d}^{*}(1, E 1) A_{f}(2, M 1)\right) \\
& -0.34 \operatorname{Re}\left(i A_{d}^{*}(1, E 1) A_{p}(2, E 2)\right)-1.01 \operatorname{Re}\left(i A_{d}^{*}(1, E 1) A_{f}(2, E 2)\right)
\end{aligned}
$$


$-0.34 \operatorname{Re}\left(i A_{p}^{*}(1, E 2) A_{d}(2, E I)\right)+0.41 \operatorname{Re}\left(i A_{f}^{*}(1, E 2) A_{d}(2, E 1)\right)$

$+0.58 \operatorname{Re}\left(i A_{d}^{*}(2, E 1) A_{p}(2, M 1)\right)-0.71 \operatorname{Re}\left(i A_{d}^{*}(2, E 1) A_{f}(2, M 1)\right)$

$\left.-0.67 \operatorname{Re}\left(i A_{d}^{*}(2, E 1) A_{p}(2, E 2)\right)-0.34 \operatorname{Re}\left(i A_{d}^{*}(2, E 2) A_{f}(2, E 2)\right)\right]$

$\times(-\sqrt{3 / 4} \sin \theta)+2\left[0.24 \operatorname{Re}\left(i A_{S}^{*}(1, E 1) A_{d}(1, E 1)\right)\right.$

$-0.24 \operatorname{Re}\left(i A_{s}^{*}(1, E 1) A_{d}(2, E 1)\right)+0.34 \operatorname{Re}\left(i A_{d}^{*}(1, E 1) A_{d}(2, E 1)\right)$

$-0.43 \operatorname{Re}\left(i A_{p}^{*}(1, M 1) A_{p}(1, E 2)\right)+0.53 \operatorname{Re}\left(i A_{p}^{*}(1, M 1) A_{f}(1, E 2)\right)$

$-0.15 \operatorname{Re}\left(i A_{p}^{*}(1, M 1) A_{p}(2, M 1)\right)+0.18 \operatorname{Re}\left(i A_{p}^{*}(1, M 1) A_{f}(2, M 1)\right)$

$-0.87 \operatorname{Re}\left(i A_{p}^{*}(1, M 1) A_{p}(2, E 2)\right)-0.43 \operatorname{Re}\left(i A_{p}^{*}(1, M 1) A_{f}(2, E 2)\right)$

$-0.16 \operatorname{Re}\left(i A_{p}^{*}(1, E 2) A_{f}(1, E 2)\right)-0.77 \operatorname{Re}\left(i A_{p}^{*}(1, E 2) A_{p}(2, M 1)\right)$

$-0.24 \operatorname{Re}\left(i A_{p}^{*}(1, E 2) A_{f}(2, M 1)\right)-0.56 \operatorname{Re}\left(i A_{p}^{*}(1, E 2) A_{p}(2, E 2)\right)$

$-0.08 \operatorname{Re}\left(i A_{p}^{*}(1, E 2) A_{f}(2, E 2)\right)+0.55 R e\left(i \dot{A}_{f}^{*}(1, E 2) A_{p} \cdot(2, M 1)\right)$

$+0.77 \operatorname{Re}\left(i A_{f}^{*}(1, E 2) A_{f}(2, M 1)\right)-0.29 \operatorname{Re}\left(i A_{f}^{*}(1, E 2) A_{p}(2, E 2)\right)$

$-0.39 \operatorname{Re}\left(i A_{f}^{*}(1, E 2) A_{f}(2, E 2)\right)-0.41 \operatorname{Re}\left(i A_{p}^{*}(2, M 1) A_{f}(2, M 1)\right)$

$-0.19 \operatorname{Re}\left(i A_{p}^{*}(2, M 1) A_{p}(2, E 2)\right)-0.58 \operatorname{Re}\left(i A_{p}^{*}(2, M 1) A_{f}(2, E 2)\right)$

$-0.55 \operatorname{Re}\left(i A_{f}^{*}(2, M 1) A_{p}(2, E 2)\right)+0.32 \operatorname{Re}\left(i A_{f}^{*}(2, M 1) A_{f}(2, E 2)\right)$

$\left.+0.40 \operatorname{Re}\left(i A_{p}^{*}(2, E 2) A_{f}(2, E 2)\right)\right](-\sqrt{15 / 16} \sin 2 \theta)+2[0.35$

$\times \operatorname{Re}\left(i A_{S}^{*}(1, E 1) A_{f}(1, E 2)\right)-0.42 \operatorname{Re}\left(i A_{S}^{*}(1, E 1) A_{f}(2, E 2)\right)$

$-0.36 \operatorname{Re}\left(i A_{d}^{*}(1, E 1) A_{p}(1, E 2)\right)-0.05 \operatorname{Re}\left(i A_{d}^{*}(1, E 1) A_{f}(1, E 2)\right)$

$-0.36 \operatorname{Re}\left(i A_{d}^{*}(1, E 1) A_{p}(2, E 2)\right)+0.42 \operatorname{Re}\left(i A_{d}^{*}(1, E 1) A_{f}(2, E 2)\right)$ 


$$
\begin{aligned}
& -0.36 \operatorname{Re}\left(i A_{p}^{*}(1, E 2) A_{d}(2, E 1)\right)+0.44 \operatorname{Re}\left(i A_{f}^{*}(1, E 2) A_{d}(2, E 1)\right) \\
& \left.-0.12 \operatorname{Re}\left(i A_{d}^{*}(2, E 1) A_{p}(2, E 2)\right)-0.06 \operatorname{Re}\left(i A_{d}^{*}(2, E 1) A_{f}(2, E 2)\right)\right] \\
& \times(-\sqrt{21 / 512})(5 \sin 3 \theta+\sin \theta)+2\left[0 . 5 3 \operatorname { R e } \left(i A_{p}^{*}(1, E 2)\right.\right. \\
& \left.\times A_{f}(1, E 2)\right)-0.65 \operatorname{Re}\left(i A_{p}^{*}(1, E 2) A_{f}(2, E 2)\right)-0.54 \\
& \times \operatorname{Re}\left(i A_{f}^{*}(1, E 2) A_{p}(2, E 2)\right)+0.53 \operatorname{Re}\left(i A_{f}^{*}(1, E 2) A_{f}(2, E 2)\right) \\
& \left.+0.22 \operatorname{Re}\left(i A_{p}^{*}(2, E 2) A_{f}(2, E 2)\right)\right](-\sqrt{45 / 2048}) \\
& \times(7 \sin 4 \theta+2 \sin 2 \theta)\}
\end{aligned}
$$

From eqs. AIV.1,AIV. 4 and AIV. 5 we may obtain the neutron polarization explicitly. 


\section{APPENDIX V}

Calculation of the Neutron Polarization for a Transition to the $6.17 \mathrm{MeV}$ Excited State of $0^{15}$

We consider only neutrons of energy 3-4 MeV, which arise either from a $19.4 \mathrm{MeV}$ photon absorption by $0^{16}$ and a subsequent neutron emission to the $0^{15} \mathrm{~g} . \mathrm{s}$., or from a 25.6 MeV photon absorption and subsequent neutron emission to the $6.17 \mathrm{MeV}$ state of $0^{15}$. From the $45^{\circ}$ polarization measurements with $24 \mathrm{MeV}$ bremsstrahlung we obtain $\mathrm{P}_{\mathrm{gs}}(19.4)=+0.24 \pm 0.24$ for the g.s. neutron polarization. The polarization measurements for $30 \mathrm{MeV}$ bremsstrahlung yield $\mathrm{P}_{\text {tot }}=$ $-0.21 \pm 0.09$ for $3-4 \mathrm{MeV}$ neutrons, but this is for a mixture of g.s. and excited state transition neutrons. We have then

$$
\mathrm{P}_{\text {tot }}=\frac{\mathrm{N}_{\gamma}(19.4) \frac{\mathrm{d} \sigma}{\mathrm{d} \Omega} \mathrm{gs}{ }^{(19.4)} \mathrm{P}_{\mathrm{gs}}(19.4)+\mathrm{N}_{\gamma}(25.6) \frac{\mathrm{d} \sigma}{\mathrm{d} \Omega} \mathrm{gs}^{(25.6)} \mathrm{P}_{\mathrm{ex}}(25.6)}{\mathrm{N}_{\gamma}(19.4) \frac{\mathrm{d} \sigma}{\mathrm{d} \Omega} \mathrm{gs}(19.4)+\mathrm{N}_{\gamma}(25.6) \frac{\mathrm{d} \sigma}{\mathrm{d} \Omega} \operatorname{ex}(25.6)}
$$

AV. 1

where $\frac{\mathrm{d} \sigma}{\mathrm{d} \Omega} \mathrm{g} \dot{\mathrm{s}}, \mathrm{P}_{\mathrm{gs}}, \frac{\mathrm{d} \sigma}{\mathrm{d} \Omega} \mathrm{ex}, \mathrm{P}_{\text {ex }}$, are defined to be the $45^{\circ}$ differential cross sections and polarizations for g.s. and $6.17 \mathrm{MeV}$ excited state transitions, and $\mathrm{N}_{\gamma}$ (19.4) and $\mathrm{N}_{\gamma}(25.6)$ are the relative photon intensities at $19.4 \mathrm{MeV}$ and 25.6 MeV. Eq. AV.1 yields

$$
P_{\text {ex }}^{\cdot}(25.6)=P_{\text {tot }}[1+r]-P_{g s}(19.4) r \quad A V .2 \text {. }
$$

where

$$
r=\frac{N_{\gamma}(19.4) \frac{d \sigma}{d \Omega} g s(19.4)}{N_{\gamma}(25.6) \frac{d \sigma}{d \Omega} \operatorname{ex}(25.6)} \quad \text { AV.3 }
$$


For a $30 \mathrm{MeV}$ thick-target bremsstrahlung beam the photon intensity ratio is $(65)$

$$
\left.\frac{\mathrm{N}_{\gamma}(19.4)}{\mathrm{N}_{\gamma}(25.6)}\right|_{\begin{array}{l}
30 \mathrm{MeV} \\
\text { brems. }
\end{array}} \simeq 1.72
$$

and it is only necessary to obtain the cross section ratio to find $\mathrm{P}_{\mathrm{ex}}(25.6)$.

The total $0^{16}(\gamma, n)$ cross section measurements of Caldwell et al (22) yield the total cross section for the $6.17 \mathrm{MeV}$ excited state transitions as $\sigma_{\text {tot }}(25.6)=4 \mathrm{mb}$. If we use the angular distribution data of Verbinski and Courtney $(26)$ to assign a $\left(1+\frac{3}{2} \sin ^{2} \theta\right)$ angular distribution to this cross section we obtain a $90^{\circ}$ cross section of $\left.\frac{\mathrm{d} \sigma}{\mathrm{d} \Omega} \operatorname{ex}(25.6)\right|_{90^{\circ}}=400 \mathrm{ub} / \mathrm{st}$. The data of Caldwell et al do not yield a g.s. cross section for $19.4 \mathrm{MeV}$ photons, but we may obtain it from the measured cross section of Verbinski and Courtney which yields approximately $200 \mathrm{ub} / \mathrm{st}$ for neutrons of 3-4 MeV energy, assuming only g.s. transitions with a $34 \mathrm{MeV}$ bremsstrahlung beam. We have the relation

$$
\frac{\mathrm{d} \sigma}{\mathrm{d} \Omega} \text { me as }\left.\right|_{90^{\circ}}=\frac{\left.\left.\mathrm{N}_{\gamma}(19.4) \frac{\mathrm{d} \sigma}{\mathrm{d} \Omega} \mathrm{gs}(19.4)\right|_{90^{\circ}+\mathrm{N}_{\gamma}(25.6) \frac{\mathrm{d} \sigma}{\mathrm{d} \Omega} \mathrm{ex}(25.6)}\right|_{90^{\circ}}}{\mathrm{N}_{\gamma}(19.4)+\mathrm{N}_{\gamma}(25.6)}
$$

AV. 5

or

$$
\left.\frac{\mathrm{d} \sigma}{\mathrm{d} \Omega} \mathrm{gs}(19.4)\right|_{90^{\circ}}=\frac{\mathrm{d} \sigma}{\mathrm{d} \Omega} \operatorname{meas}\left|90^{\circ}\left[\mathrm{r}^{\prime}+1\right]-\frac{\mathrm{d} \sigma}{\mathrm{d} \Omega} \operatorname{ex}(25.6)\right|_{90^{\circ}\left[\mathrm{r}^{\prime}\right]}
$$

AV. 6

where 


$$
r^{\prime}=\frac{N_{\gamma}(25.6)}{N_{\gamma}(19.4)} \mid \begin{aligned}
& 34 \mathrm{MeV} \\
& \text { brems }
\end{aligned}
$$

$\simeq 0.64$

AV. 7

Using the measured cross sections this gives

$$
\left.\frac{\mathrm{d} \sigma}{\mathrm{d} \Omega} \mathrm{gs}(19.4)\right|_{90^{\circ}}=72 \mathrm{ub} / \mathrm{st} \quad \text { AV. } 8
$$

and

$$
\left.\frac{\frac{\mathrm{d} \sigma}{\mathrm{d} \Omega} g s(19.4)}{\frac{d \sigma}{d \Omega} \operatorname{ex}(25.6)}\right|_{90^{\circ}}=0.18 \quad \text { AV.9 }
$$

Assuming that the $45^{\circ}$ and $90^{\circ}$ cross sections have the same ratio, eqs. AV.9, AV.2, AV. 3 and AV.4 yield

$$
\left.\mathrm{P}_{\text {ex }}(25.6)\right|_{45^{\circ}}=-0.35 \pm 0.20 \quad \text { AV. } 10
$$

where the error is calculated only from the errors in the polarization measurements. The results AV.10 have a larger error than given because of uncertainties in the cross section ratio at $45^{\circ}$ and in the crude estimates used for the photon intensity ratios. 


\section{B I BLI OGRAPHY}

1. E. Hayward, Rev. Mod. Phys., 35, 324 (1963)

2. E. Hayward; Nuclear Structure and Electromagnetic Interactions, N. MacDonald, ed., Plenum Press, N.Y., 1962 pp. 141-210.

3. M. Goldhaber and E. Teller, Phys. Rev., 74, 1046 (1948).

4. H. Steinwedel and J.H.P. Jensen, Z. Naturforschung, 5a, 413 (1950).

5. D.H. Wilkinson, Physica, 22, 1039 (1956).

6. J.P. Elliott and B.H. Flowers, Proc. Roy. Soc. London, 242A, 57 (1957).

7. N.A. Burgov, G.V. Danilyan, B.S. Dolbilkin, L.E. Lazareva and F.A. Nikolaev, Zhur. Eksptl. i. Teor. Fiz., 43, 70 (1962), and Soviet Physics - JETP, 16, 50 (1963).

8. G.E. Brown and M. Bolsterli, Phys. Rev. Lett., $\underline{3}, 472$ (1959).

9. G.E. Brown, L. Castillejo and J.A. Evans, Nucl. Phys., 22, I (1961).

10. N. Vinh-Mau and G.E. Brown, Nucl. Phys., 29, 89 (1962).

11. V. Gillet and N. Vinh-Mau, Nuc1. Phys., 54, 321(1964).

12. V.V. Balashov, V.G. Shevchenko and N.P. Yudin, Nucl.Phys., $27,323(1961)$.

13. H.P. Jo11y, Nuc1. Phys., 67, 209 (1965).

14. F. Villars and M.S. Weiss, Phys. Lett., 11, 318 (1964).

15. M.S. Weiss, Phys. Lett., 19, 393 (1965).

16. B. Buck and A.D. Hill, To Be Published.

17. J. Goldemberg and W.C. Barber, Phys. Rev., 134, B963 (1964).

18. D.B. Isabe1le and G.R. Bishop, J.De Phys. Et.Rad., 22, 548(1961).

19. G.R. Bishop, B. Grossetête and J.C. Risset, J. De Phys.Et.Rad.; 23, $31(1962)$.

20 G.J. Vanpraet, Nucl.Phys., 74, 219 (1965).

21 G.J. Vanpraet and W.C. Barber, Nucl. Phys., 79, 550(1966). 
22. J.T. Caldwe11, R.L. Bramblett, B.L. Berman, R.R. Harvey and S.C. Fultz, Phys.Rev.Lett., 15, 976(1965).

23. E. Hayward and T. Stovall, Nucl. Phys., 69, $241(1965)$.

24. F.W.K. Firk and K.H. Lokan, Phys. Rev.Lett., 8, $321(1962)$.

25. P.F. Yergin, R.H. Auguston, N.N. Kausha1, H.A. Medicus,

W.R. Moyer and E.J. Winhold, Phys. Rev. Lett., 12, $733(1964)$.

26. V.V. Verbinski and J.C. Courtney, Nuc1. Phys., 73, 398(1965).

27. S.A.E. Johansson and B. Forkman, Ark. F. Fys., 12, 359(1957).

28. N.W. Tanner, G.C. Thomas and E.D. Earle, Proc, Ruth..Jub. Int.Conf., Paris, Ju1y, 1964, Vo1. II, pp. 385-6.

30. N.W. Tanner, G.C. Thomas and E.D. Earle, Nucl.Phys., 52, $45(1964)$.

31. S.G. Cohen, P.S. Fisher and E.K. Warburton, Phys.Rev.lett., 3, $433(1959)$.

32. N.W. Tanner, G.C. Thomas and W.E. Meyerhof, Nuovo Cim., 14, 257 (1959).

33. W.R. Dodge and W.C. Barber, Phys.Rev.; 127, 1746 (1962).

34. N.A. Burgov, G.W. Danilyan, B.S. Dolbilkin, L.E. Lazareva' and F.A. Nikolaev, Zhur. Ekspt1.i.Teor.Fiz., 45, 1693(1963), and Soviet Physics - JETP, 18, 1159(1964).

35. S.C. Fu1tz, J.T. Caldwe11, B.L. Berman, R.L. Bramblett and R.R. Harvey, Phys. Rev., 143, $790(1966)$.

36. F.R. Allum, T.W. Quirk and B.M. Spicer, Nucl.Phys., 53,545 $(1964)$.

37. H.E. Gove, A.E. Litherland and R. Batchelor, Nucl.Phys., 26, $480(1961)$.

38. R.G. Allas, S.S. Hanna, L. Meyer-Schutzmeister and R.E. Segal, Nuc1. Phys., 58, 122(1964).

39. L.I. Schiff, Phys. Rev., 96, 765 (1956)

40. R.S. Willey, Nucl. Phys., 40, $529(1963)$.

41. F.H. Lewis, Jr. Phys. Rev., 134, B331 (1964).

42. F.H. Lewis, Jr. and J.D. Walecka, Phys. Rev., 133, B849(1964).

43. S. Penner and J.E. Leiss, Phys. Rev., 114, 1101 (1959).

44. B. Bosco and S. Fubini, Nuovo Cim., 9, 350 (1958).

45. F.H. Lewis, Jr., Nucl.Phys., 80, 465 (1965). 
46. S.B. Kowalski, Ph.D. Thesis (Unpub) Physics, MIT, Jan. 1963.

47. R.A. Nobles, Rev. Sci.Inst., 27, 280 (1956).

48. A. Sayres and C.S. Wu, Rev. Sci. Inst., 28, 758 (1.957).

49. J.A. Northrop and J.M. Gursky, Nucl.Inst., $\underline{3}, \quad 207(1958)$.

50. J.A. Northrop, Rev. Sci.Inst., 29, 437 (1958).

51. J.R. Kane, R.T.Siege1 and A. Suzuki, Rev. Sci.Inst., 34, 817 (1963).

52. Q.A. Kerns and G.C. Cox, Nuc1.Inst. Meth., 12, 32(1961). PEK-118-TR Lamps obtained from PEK Labs. Inc. Sunnyvale, Calif.

53. B. Hoop, Jr. and H.H. Barscha11, The Analyzing Power of Neutrons for Helium, From International Conference on Polarization Phenomena of Nucleons, Karlsruhe, Germany, 1965.

54. J.D. Seagrave, Phys.Rev., 92, 1222 (1953).

55. V.V. Verbinski, J.C. Courtney, W.R. Burrus and T.A. Love, The Response of Some Organic Scintillators to Fast Neutrons (ORNL-P-993), From American Nuclear Society Meeting, San Francisco, Nov-Dec. 1964.

56. R.W. Batchelor, W.B. Gilboy, J.B. Parker and J.H. Towle, Nucl. Inst. Meth., 13, $70(1961)$.

57. W.S.C. Williams, An Introduction to Elementary Particles, Academic Press, New York, 1961.

58. G.S. Mutchler, Ph.D. Thesis (Ụnpub) Physics, MIT, Oct. 1965.

59. J.E. Wills, Jr., J.K. Bair, H.O. Cohn and H.B. Willard, Phys. Rev., 109, 891(1958).

60. R.W. Meier, P. Scherrer and G. Trumpy, Helv. Phys.Acta., 27, 577 (1954).

61. S.G. Buccino, C.E. Hollandsworth, H.W. Lewis and P.R.Bevington Nucl. Phys., 60, 17(1964).

62. E.Erba, U. Facchini and E.S. Menichel1a, Nuovo Cim., 22, 1237 (1961).

63. D.W. Lang, Nuc1.Phys., 26, 434 (1961).

64. D.B. Thomson, Phys. Rev., 129, 1649 (1963).

65. H.W. Koch and J.W. Motz, Rev. Mod. Phys., 31, 920 (1959).

66. A.M. Baldin, V.I. Gol'danskii and I.L. Rozenthal, Kinematics of Nuclear Reactions, Pergamon Press, New York, 1961.

67. R. Raphae1 and H. Übera11, Nuc1. Phys., 85, 327(1966). 


\section{Acknowledgement}

The author expresses sincere gratitude to the many. people who have provided him with guidance and help in carrying out the research reported in this thesis. Most thanks are due to Prof. William Bertozzi, who spent much of his time supervsing and reading this thesis. Special acknowledgement must a1so go to Dr. C. P. Sargent, Dr. W. E. Turchinetz and Prof. S. B. Kowalski, as the research could not have been carried out without their valuable assistance. Gratitude must also be expressed to Dr. J. Russell and Dr. R. Fullwood of RPI, and to Prof. N. C. Rasmussen of the MIT Nuclear Engineering Dept.

The assistance of Prof. P. T. Demos of MIT and of Dr. E. R. Gaerttner of RPI is acknowledged, as is the help of the graduate students at the MIT Linear Accelerator Group, especially T. W. Phillips, Miss J. L. Matthews, and Dr. G. S. Mutchler. Many thanks go to G. Sechen for help with moving and setting up equipment, and many other details. The assistance of R. Rudzinski with some of the tests with the Cockroft-Walton is acknowledged. Gratitude is expressed to the many people in the Laboratory for Nuclear Science at MIT, who helped in various portions of the research.

I. would also like to thank my room-mates, W. E. Couch and M. J. Lysaght, for their helpful criticism of various aspects of the final stage of the thesis. Many thanks are due to Miss J. Holloway who did most of the typing of the final copy of the thesis. Finally I would like to thank my fiance, Miss Anne N. Forde, for the encouragement she has given me. 